\title{
Orthogonal Supramolecular
}

\section{Interaction Motifs for Functional \\ Monolayer Architectures}


ORTHOGONAL SUPRAMOLECULAR INTERACTION MOTIFS FOR FUNCTIONAL MONOLAYER ARCHITECTURES 
This research has been financially supported by the Council for Chemical Sciences of the Netherlands Organization for Scientific Research (NWO-CW), (grant 700.55.029 to Jurriaan Huskens). The research was carried out within the Molecular Nanofabrication (MnF) group, $\mathrm{MESA}^{+}$Institute for Nanotechnology, University of Twente.

Publisher: Wöhrmann Print Service, Zutphen, The Netherlands

(C) Mahmut Deniz Yilmaz, Enschede, 2011

No part of this work may be reproduced by print, photocopy or any other means without the permission in writing of the author.

ISBN 978-90-365-3205-1 


\section{ORTHOGONAL SUPRAMOLECULAR INTERACTION MOTIFS FOR FUNCTIONAL MONOLAYER ARCHITECTURES}

PROEFSCHRIFT

ter verkrijging van

de graad van doctor aan de Universiteit Twente,

op gezag van de rector magnificus,

prof. dr. H. Brinksma,

volgens besluit van het College voor Promoties

in het openbaar te verdedigen

op donderdag 9 juni 2011 om 14.45 uur

door

Mahmut Deniz Yilmaz

geboren op 14 april 1979

te Ankara, Turkije 
Dit proefschrift is goedgekeurd door:

Promotor: $\quad$ Prof. dr. ir. Jurriaan Huskens 
To my wife, grandma, parents and all my family 



\section{Table of Contents}

Chapter 1 General introduction

Chapter 2 Orthogonal Supramolecular Interaction Motifs for Functional 5 Monolayer Architectures

2.1. Introduction

2.2. Hydrogen bonding directed assembly on surfaces 7

$\begin{array}{ll}\text { 2.2.1 Assembly of molecules on SAMs } & 7\end{array}$

2.2.2 Assembly of nanoparticles on SAMs 11

2.3. Metal coordination directed assembly 12

$\begin{array}{ll}\text { 2.3.1 Assembly of molecules on SAMs } & 12\end{array}$

2.3.2 Assembly of nanoparticles on SAMs $\quad 15$

2.4. Assembly by electrostatic interactions 18

2.4.1 Assembly of molecules on SAMs 18

2.4.2 Assembly of nanoparticles on SAMs 20

2.5. Assembly by host-guest interactions 22

2.5.1 Assembly of molecules on SAMs 22

2.6. Combination of different orthogonal supramolecular interaction 25 motifs

2.7. Conclusions 33

2.8. References 34

Chapter 3 Expression of Sensitized $\mathrm{Eu}^{3+}$ Luminescence at a Multivalent 41 Interface

3.1. Introduction 44

3.2. Results and Discussion 63

3.2.1 Synthesis 44

3.2.2 Complex formation in solution $\quad 47$

3.2.3 Complex formation at the molecular printboard 48

3.3. Conclusions $\quad 54$ 
3.4. Acknowledgements

3.5. Experimental Section $\quad 55$

3.6. References 63

Chapter 4 Ratiometric Fluorescent Detection of an Anthrax Biomarker at 65 Molecular Printboards

4.1. Introduction 66

4.2. Results and discussion 67

4.3. Conclusions 75

4.4. Acknowledgements 76

4.5. Experimental section $\quad 76$

$\begin{array}{ll}\text { 4.6. References } & 78\end{array}$

Chapter 5 A Supramolecular Sensing Platform in a Microfluidic Chip $\quad 81$

5.1. Introduction 82

5.2. Results and Discussion 84

5.2.1 Fabrication of the Sensing Platform and Anion Detection 84

5.2.2 Sensing of Biologically Relevant Phosphates 87

5.2.3 Screening of an Antrax Biomarker and Potentially Interfering 91 Anions

5.3. Conclusions 95

5.4. Acknowledgements 96

5.5. Experimental section 96

$\begin{array}{ll}\text { 5.6. References } & 98\end{array}$

Chapter 6 Local Doping of Silicon Using Nanoimprint Lithography and 103 Molecular Monolayers

6.1. Introduction 104

6.2. Results and discussion 106

6.2.1 NIL-patterned monolayers on silicon 106

6.2.2 Local doping of silicon by NIL-patterning, monolayer 114 formation and rapid thermal annealing

6.3. Conclusions 
6.4. Experimental section 123

6.5. Acknowledgements 123

6.6. References 129

$\begin{array}{lll}\text { Chapter } 7 & \text { Fabrication of Two-Dimensional Organic Spin Systems on Gold } 133\end{array}$

7.1. Introduction 134

7.2. Results and Discussion 136

7.2.1 Monolayer fabrication and characterization 136

7.2.1.1 Characterization of terpyridinyl-metal SAMs on gold $\quad 138$

7.2.1.2 Characterization of TEMPO SAMs on gold 142

7.2.2 Electrical characterization $\quad 145$

7.2.2.1 Characterization of Co(Tpy-SH $)_{2}$ SAMs on gold $\quad 146$

7.2.2.2 Characterization of Co(Tpy)(Tpy-SH) SAM on gold 148

7.2.2.3 Characterization of TEMPO SAMs on gold 150

7.3. Conclusions 152

7.4. Acknowledgements 152

7.5. Experimental section 153

7.6. References 154

$\begin{array}{ll}\text { Summary } & 157\end{array}$

$\begin{array}{ll}\text { Samenvatting } & 161\end{array}$

$\begin{array}{ll}\text { Acknowledgements } & 167\end{array}$

$\begin{array}{ll}\text { About the author } & 171\end{array}$ 


\section{Chapter 1}

\section{General Introduction}

Supramolecular chemistry and self-assembly processes have evolved to be one of the most important fields in modern chemistry of the last two decades. ${ }^{[1]}$ Molecular recognition and self-assembly represent the basic concept of supramolecular chemistry and involved noncovalent interactions. ${ }^{[2]}$ Noncovalent interactions (e.g. hydrogen bonding, metal-ligand coordination, electrostatic, and host-guest interactions) are usually weaker than covalent bonds and they are reversible. The use of supramolecular interactions to direct the spontaneous assembly of molecules is of utmost importance due to their high specificity, controlled affinity, and reversibility. ${ }^{[3]}$ These specific and highly controllable interactions can be manipulated independently and simultaneously, providing orthogonal self-assembly which describes the assembly of components with multiple (i.e. more than one) interaction motifs that do not influence each other's assembly properties, applied in the same system. ${ }^{[4]}$

Today a variety of orthogonal supramolecular systems are known in solution. ${ }^{[5]}$ Although these weak interactions were employed individually to build supramolecular architectures on surfaces, few attempts have been reported for the generation of hybrid, multifunctional materials based on orthogonal interactions. The research described in this thesis is focused on the combination of these interactions (orthogonal supramolecular interactions) for functional monolayer architectures on surfaces.

In Chapter 2 of this thesis, a literature overview is given regarding the use of individual supramolecular interaction motifs (hydrogen bonding, metal coordination, 
electrostatic and host-guest interactions) for assembly on surfaces as well as recent studies describing the combination of these interactions.

The first part of the thesis (Chapters 3, 4 and 5) deals with the multivalent binding of supramolecular complexes at molecular printboards which are monolayers of cyclodextrin (CD) on a surface. Chapter 3 describes the combination of orthogonal host-guest and lanthanide-ligand coordination interaction motifs. Antenna-sensitized $\mathrm{Eu}^{3+}$ luminescence based on host-guest interactions on the molecular printboard is employed for qualitative and quantitative studies of the complexation of different building blocks.

In Chapter 4, the same lanthanide complex system is used for the ratiometric detection of dipicolinic acid (DPA), which is a unique biomarker for anthrax bacterial spores, on a receptor surface. The system constitutes the first lanthanide-based surface receptor system for the detection of DPA.

Chapter 5 continues the study described in Chapter 3 and 4. By using the same lanthanide complex system, a supramolecular high-throughput platform based on selfassembled monolayers implemented in a microfluidic device is described resulting in a general detection method for biologically relevant phosphate anions and DPA.

The second part of the thesis (Chapters 6 and 7) concerns the use of the functional monolayers for nanoelectronics. Chapter 6 introduces the local doping of oxide-free silicon using nanoimprint lithography (NIL) and molecular monolayers. Covalently bonded Si-C monolayer patterns with feature sizes ranging from $100 \mathrm{~nm}$ to $100 \mu \mathrm{m}$ are created via a local hydrosilylation reaction on NIL-patterned resist areas. This novel patterning strategy is successfully applied for introducing dopant atoms in the underlying silicon substrate using a phosphorus-containing molecular precursor on oxide-free silicon.

Chapter 7 describes the fabrication of monolayers of organic molecules with unpaired spins on a thin gold film. The existence of unpaired spins in self-assembled 
monolayers is demonstrated. Electrical transport measurements are performed and an increase of the gold film sheet resistance for temperatures below $\sim 20 \mathrm{~K}$ for some examples is observed.

\section{References}

[1] G. M. Whitesides, B. Grzybowski, Science 2002, 295, 2418-2421.

[2] J. M. Lehn, Supramolecular Chemistry, Concepts and Perspectives, VCH, Weinheim, Germany, 1995.

[3] J. M. Lehn, Rep. Prog. Phys. 2004, 67, 249-265.

[4] P. E. Laibinis, J. J. Hickman, M. S. Wrighton, G. M. Whitesides, Science 1989, 245, 845-847.

[5] H. Hofmeier, U. S. Schubert, Chem. Commun. 2005, 2423-2432. 


\section{Chapter 2}

\section{Orthogonal Supramolecular Interaction Motifs for Functional Monolayer Architectures}

This chapter gives an overview on the recent developments of orthogonal supramolecular interactions on surfaces. The first part deals with the use of noncovalent interactions, including hydrogen bonding, metal coordination, electrostatics and host-guest interactions, to modify surfaces. The second part describes the combination of different, orthogonal supramolecular interaction motifs for the generation of hybrid assemblies and materials. The integration of different supramolecular systems is essential for the self-assembly of complex architectures on surfaces. 


\subsection{Introduction}

Supramolecular chemistry refers to the area of the chemistry of molecular assemblies and of the intermolecular bond (chemistry beyond the molecule) and focuses on the development of self-assembly pathways towards large moleculer systems or molecular arrays. ${ }^{[1]}$ Molecular self-assembly has been demonstrated by supramolecular chemistry and can be defined as the spontaneous assembly of the molecules under equilibrium conditions into stable, structurally well-defined aggregates through noncovalent interactions (e.g. hydrogen bonding, metal coordination, electrostatic or host-guest interactions) which are usually weaker than covalent bonds. Moreover, supramolecular interactions are reversible, whereas covalent bonds are usually irreversible. The use of supramolecular interactions to direct the spontaneous assembly of molecules is of utmost importance owing to their high specificity, controlled affinity, and reversibility. These specific and highly controllable interactions can be manipulated independently and simultaneously, providing orthogonal selfassembly which describes the assembly of components with multiple (i.e. more than one) interaction motifs that do not influence each other's assembly properties, applied in the same system. ${ }^{[2]}$

Self-assembled monolayers (SAMs) are ordered molecular assemblies formed by the adsorption of an adsorbate onto a solid surface. SAMs provide a convenient way to produce surfaces with specific chemical functionalities. Regarding the concept of controlled positioning of molecules on a surface, binding stoichiometry, binding strength, binding dynamics, packing density and order, and reversibility serve as crucial tuning parameters. Covalent immobilization of molecules does not offer convenient versatility and flexibility over most of these parameters. Supramolecular interactions afford a solution to the control of these criteria. Hence, the orthogonal self-assembly concept, integrated with various surface patterning methods such as soft-lithography, provides rapid and site-selective adsorption of 
molecules and micrometer scale objects at predefined regions with high specificity and selectivity for the fabrication of complex hybride organic-inorganic materials.

Comprehensive reviews exist on orthogonal supramolecular interactions in solution. ${ }^{[3]}$ Objective of this chapter is to give an overview of the current understanding of orthogonal supramolecular interactions and its potential as a self-assembly tool on solid surfaces. For this reason, the focus will be on the individual supramolecular interaction motifs (hydrogen bonding, metal coordination, electrostatic and host-guest interactions) as well as recent advances for the combination of these orthogonal interactions for functional monolayer architectures on surfaces.

\subsection{Hydrogen bonding directed assembly on surfaces}

Self-assembly through multiple hydrogen bonding interactions has been widely used to create functional monolayers and new materials on surfaces. Multiple hydrogen bonding is of major importance in order to the enhanced stability of systems and allows assembly at near-equilibrium conditions, which facilitates control over the thermodynamic parameters of the assembly.

\subsubsection{Assembly of molecules on SAMs}

Rotello and co-workers have developed a method to manipulate conductance using hydrogen bonding interactions at a self-assembled monolayer surface (Figure 2.1). ${ }^{[4]} \mathrm{A}$ binder molecule, diacyl 2,6-diaminopyridine decanethiolate was inserted into a background monolayer of decanethiolate on gold using replacement lithography. Electroactive functionalization of the monolayer was then achieved through binding of the complementary ferrocene-terminated uracil to the binder molecule. The ferrocene functionality can be replaced by dodecyl uracil for erasing the conductance. Current-voltage properties of the 
patterned region were monitored by using an STM tip. Noncovalent self-assembly provides a potential method to install and subsequently remove electroactive functionality.
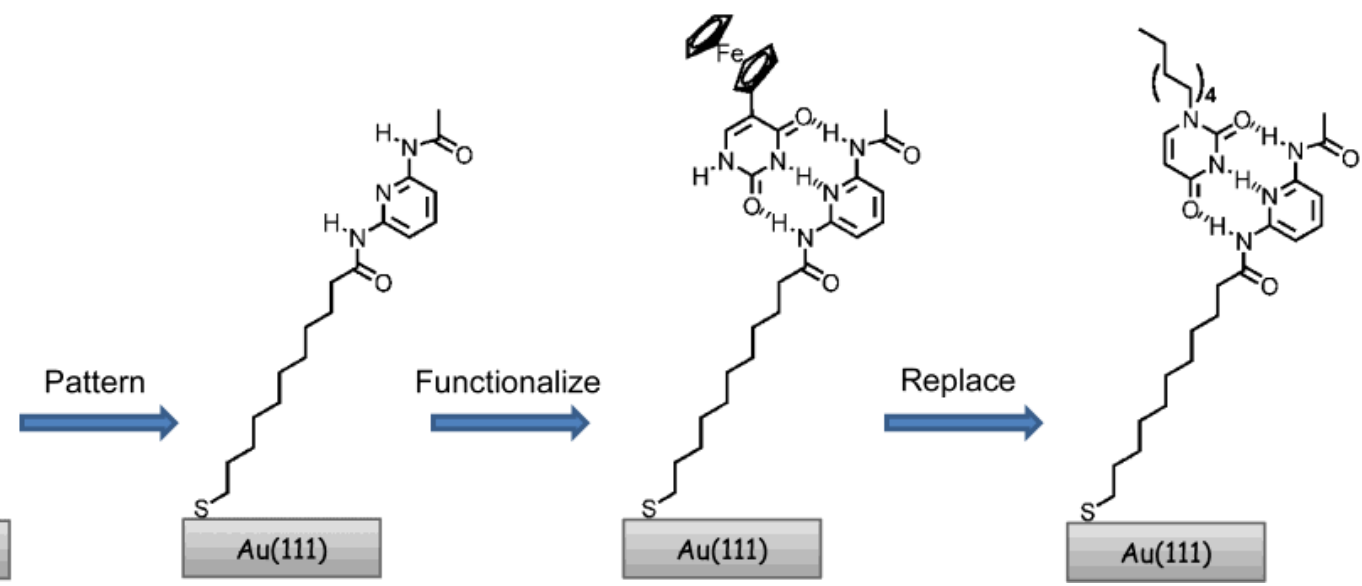

Figure 2.1 Patterning, functionalization and erasing at the surface of assembled monolayer. Adapted with permission from ref 4. Copyright 2002 American Chemical Society.

Rotello and co-workers used three-point hydrogen bonding interactions between modified SAMs and complementary functionalized mono- and di- block copolymers to direct the adsorption process onto surfaces. ${ }^{[5]}$ The thymine-diamidopyridine (Thy-DAP) hydrogen bonding motif provided a highly selective adsorption of the DAP- containing mono- and diblock copolymers onto the Thy-decorated gold surface under controlled deposition conditions (Figure 2.2). 


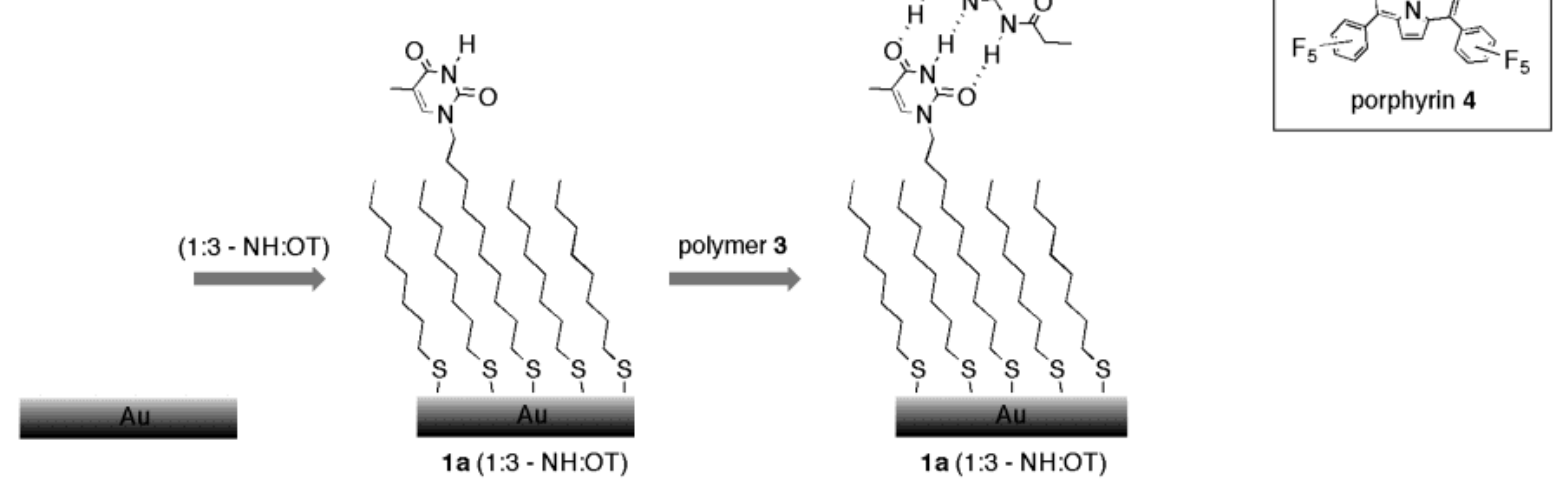

Figure 2.2 Polymers tethered to surfaces using hydrogen bonding interactions. Adapted with permission from ref 5. Copyright 2003 American Chemical Society.

The group of Cooke demonstrated that phenanthrenequinone binds strongly to ureas and thioureas by forming two hydrogen bonds which can be modulated by altering the redox state of the quinone. ${ }^{[6]}$ A SAM of a disulfide phenanthrenequinone binds phenyl urea terminated PPI dendrimers by forming multiple interactions. Upon oxidation, the dendrimers bind to the surface 2000 -fold stronger while for a monovalent model compound a smaller increase of binding strength was observed. Myles and co-workers have described the immobilization of barbituric acid derivatives on mixed monolayers of alkanethiols and bis(2,6-diaminopyridine) amide of isophthalic acid-functionalized dedecanethiol on gold films. ${ }^{[7]}$ The immobilization of barbiturate derivatives to the receptor functionalized SAM involved the use of multiple hydrogen bonds to achieve a stable assembly on the surface (Figure 2.3). 


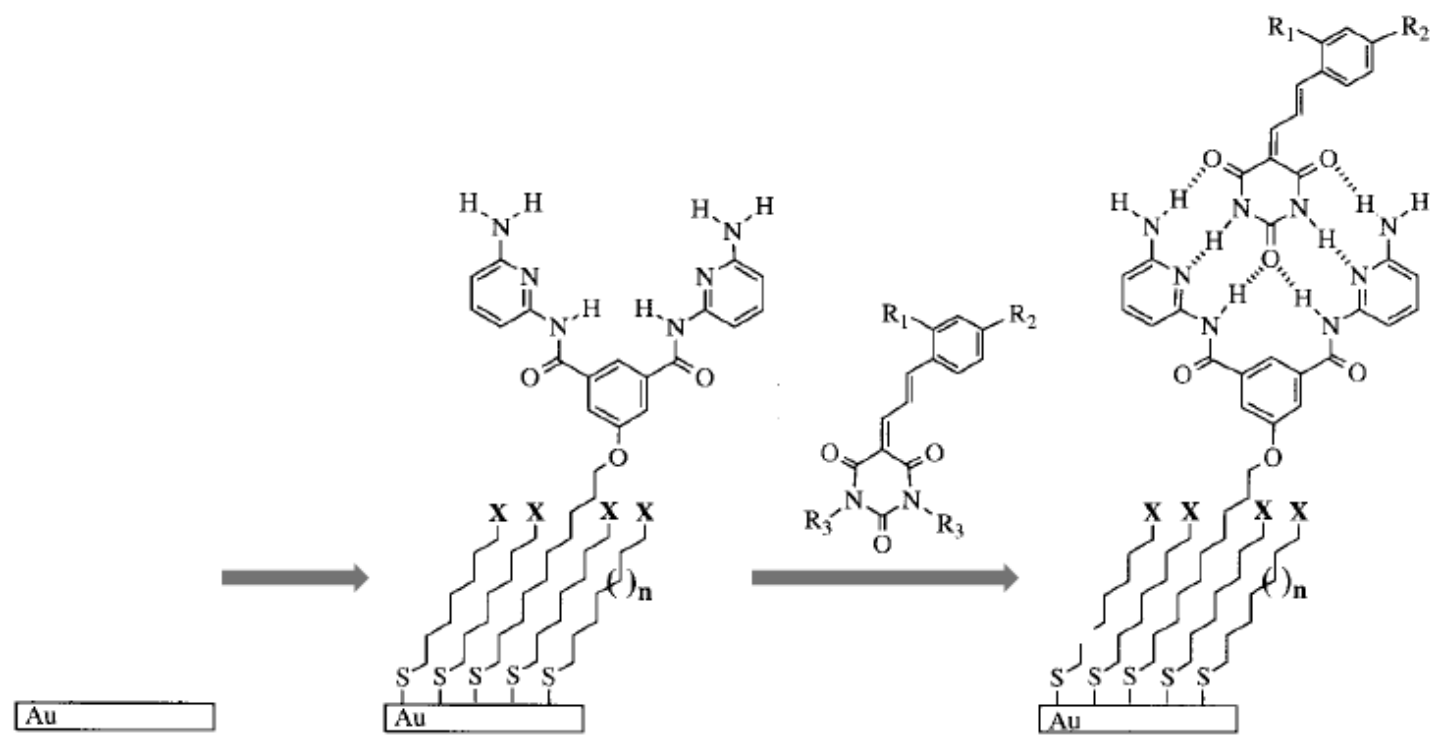

Figure 2.3 Assembly between barbituric acid derivatives and the bis(2,6-diaminopyridine) amide of isophthalic acid on a gold film. Adapted with permission from ref 7 . Copyright 1998 American Chemical Society.

Reinhoudt et al. have reported synthetic hydrogen bonded assemblies on gold surfaces. ${ }^{[8]}$ The spontaneous assembly process was performed by incorporating the thioether functionalized calix[4]arene dimelamines into a thiolate SAM. Subsequently, the monolayers containing one of the building blocks were immersed in a solution of the already formed assemblies, resulting in stable hydrogen bonded assemblies at the surface (Figure 2.4).

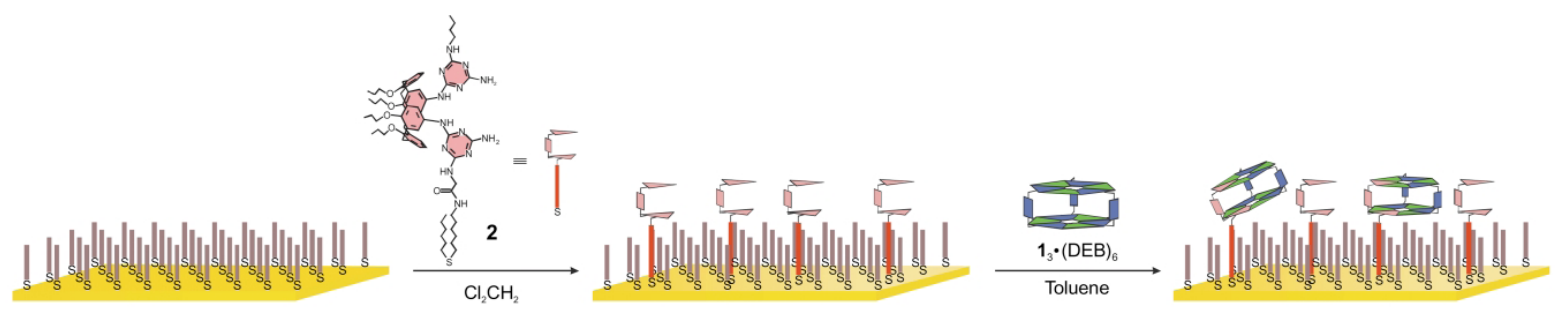

Figure 2.4 The methodology followed for the growth of assemblies $\mathbf{1}_{2}$.2.(DEB) 6 on gold. Reproduced with permission from ref 8. Copyright 2003 The Royal Society of Chemistry. 


\subsubsection{Assembly of nanoparticles on SAMs}

Binder and co-workers described an example of hydrogen bonding interaction for nanoparticle assembly on flat surfaces. ${ }^{[9]}$ The approach is based on the multiple hydrogen bonding interactions of the receptor immobilized on nanoparticles. It was found that the surface coverage of nanoparticles could be adjusted by the density of receptor units in the mixed SAM (Figure 2.5).

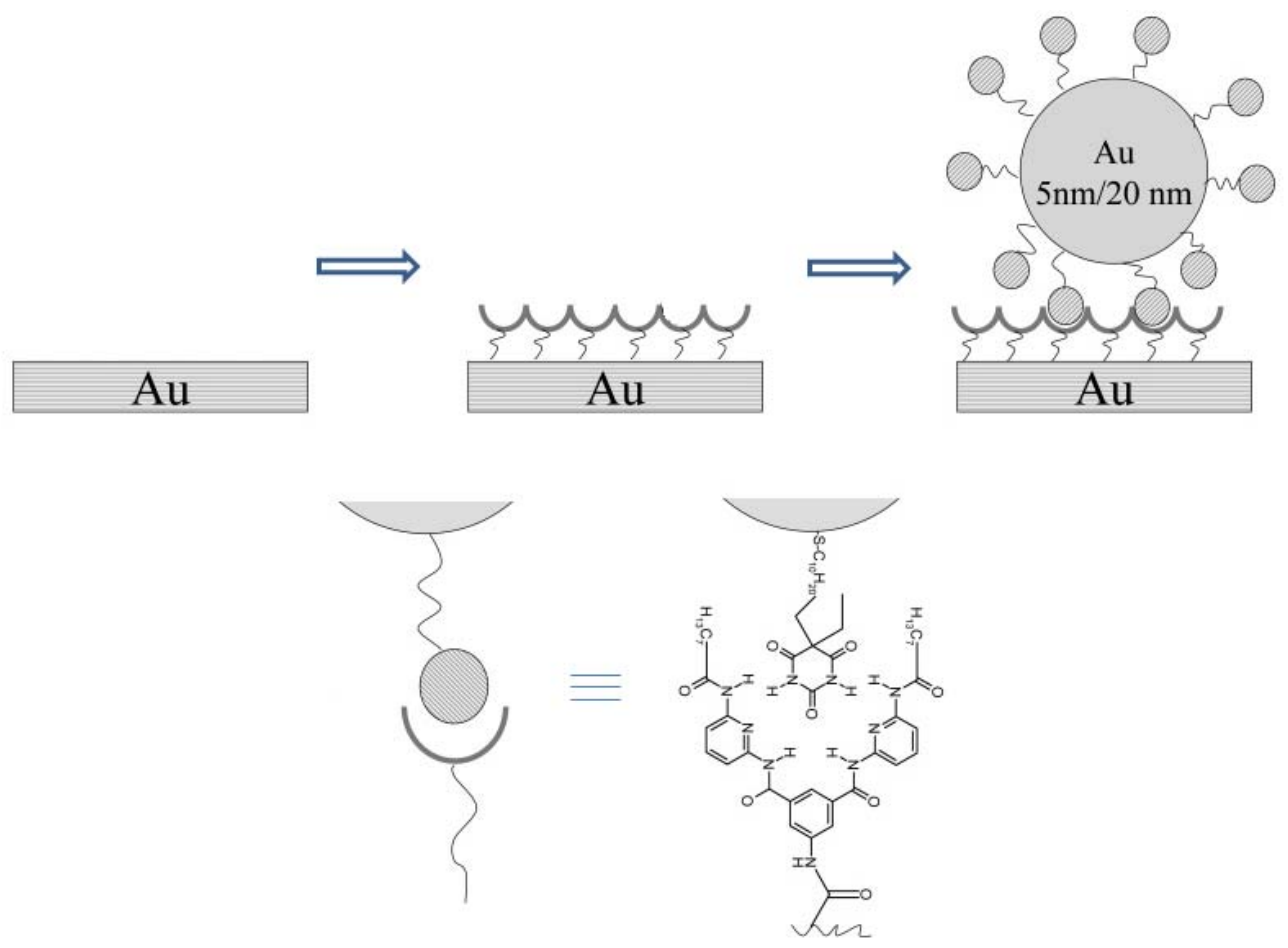

Figure 2.5 Schematic illustration of hydrogen-bonding directed nanoparticle assembly. Adapted with permission from ref 9. Copyright 2005 American Chemical Society.

Rotello and co-workers developed nanoparticle assembly on flat surfaces through specific hydrogen bonding interactions. ${ }^{[10]}$ They demonstrated the selective deposition of polystyrene functionalized with complementary diamidopyridine (PS-DAP)/ and thymine (PSThy) gels onto pre-patterned silicon substrates. These microgel arrays can be crosslinked and selectively and reversibly functionalized by nanoparticles through complementary 
hydrogen bonding interactions to provide polymer/nanoparticle composite microstructure patterns with fluorescent or magnetic properties.

The same group reported the use of electron-beam lithography (EBL) to pattern a functional polymer "host" template composed of diaminotriazine-functionalized polystyrene via electron-beam-induced cross linking. ${ }^{[11]}$ After development, the cross-linked polymer pattern provides templates for assembling complementary thymine-functionalized CdSe-ZnS quantum dots (QDs) via three point hydrogen-bonding interactions.

\subsection{Metal coordination directed assembly}

Metal directed self-assembly on surfaces has been extensively studied for the construction of supramolecular architectures. Coordination chemistry is of special interest for the assembly, because it offers stable bonding and metal-ligand specificity, also allows the reversible formation and cleavage of the complex by redox processes or the addition of competing ligands.

\subsubsection{Assembly of molecules on SAMs}

Abruna et al. has reported ligand-metal assembly on gold for the preparation of redox active mono and multimetallic systems. ${ }^{[12]}$ Study shows that the surface-bound terpyridine ligand has enough coordination sites to bind other metal ions on the surface (Figure 2.6A). A similar approach was used by Nishihara and co-workers to build polymetallic complexes on gold by repetitive deposition of an $\mathrm{Fe}(\mathrm{II})$ complex with azobenzene-linked bis(terpyridine) ligand. ${ }^{[13]}$ The group of Schubert described the use of a terpyridine-metal complex to reversibly functionalize surfaces. ${ }^{[14]}$ The optical surface properties could be tuned by the choice of the coordinating metal ion. The introduction of suitable coordinating transition metal ions allowed the reversible formation 


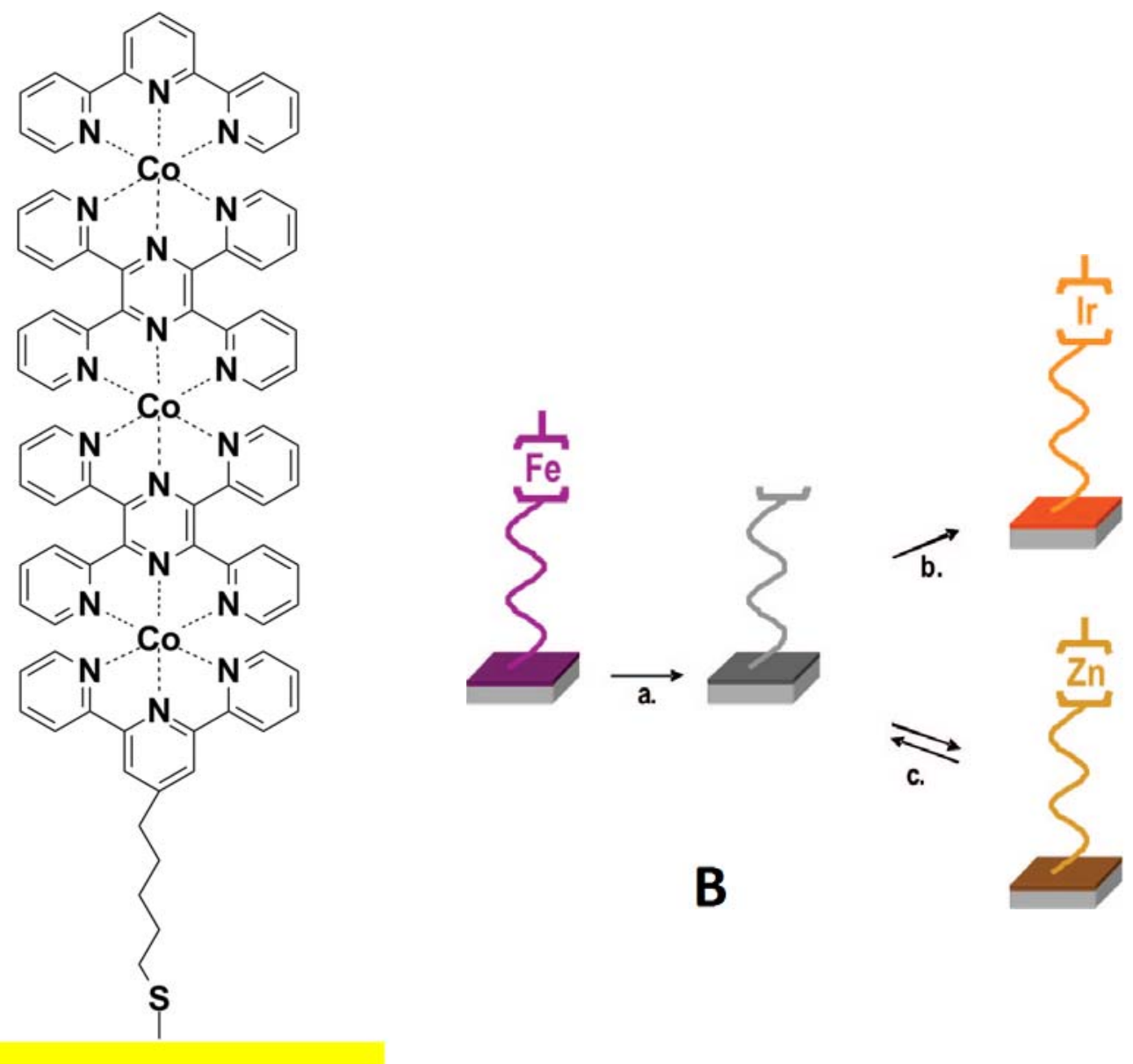

\section{A}

Figure 2.6 (A) Orthogonal assembly scheme for the construction of terpyridine-metal complex layer on gold. Adapted with permission from ref 12. Copyright 1996 American Chemical Society. (B) The attached Fe(II) complex was uncomplexed to obtain the free terpyridine ligands on the substrates (a). These units can be used for the subsequent complexation with an iridium precursor (b) or with $\mathrm{Zn}(\mathrm{II})$ ions; the latter system can be reversibly opened and closed (c). Reproduced with permission from ref 14. Copyright 2008 American Chemical Society.

and disassembly of the surface bounded complexes (Figure 2.6B). Reinhoudt and co-workers used the metal coordination to generate coordination cages directly on surfaces by using selfassembly. ${ }^{[15]}$ Metal induced coordination allowed the direct measurement of the formation of 
such assemblies and detection on a single molecule level. The same group also developed a new way to immobilize heterocages on surface by metal coordination. ${ }^{[16]}$ Immobilized heterocages result from the insertion of the thioether-functionalized cavitand into an 11mercapto undecanol SAM, followed by assembly of cages by complexation of a different cavitand from solution. A different approach for the metal coordination directed assembly was developed by Rubinstein and co-workers. ${ }^{[1]}$ Using bishydroxamate ligands and corresponding metals such as $\mathrm{Zr}^{4+}, \mathrm{Ce}^{4+}$ and $\mathrm{Ti}^{4+}$, a new type of multilayer structures based on supramolecular metal-ligand interactions has been constructed in a step by step manner, resulting a larger thickness, increased roughness, higher electrical resistivity-and improved stiffness of surfaces (Figure 2.7).
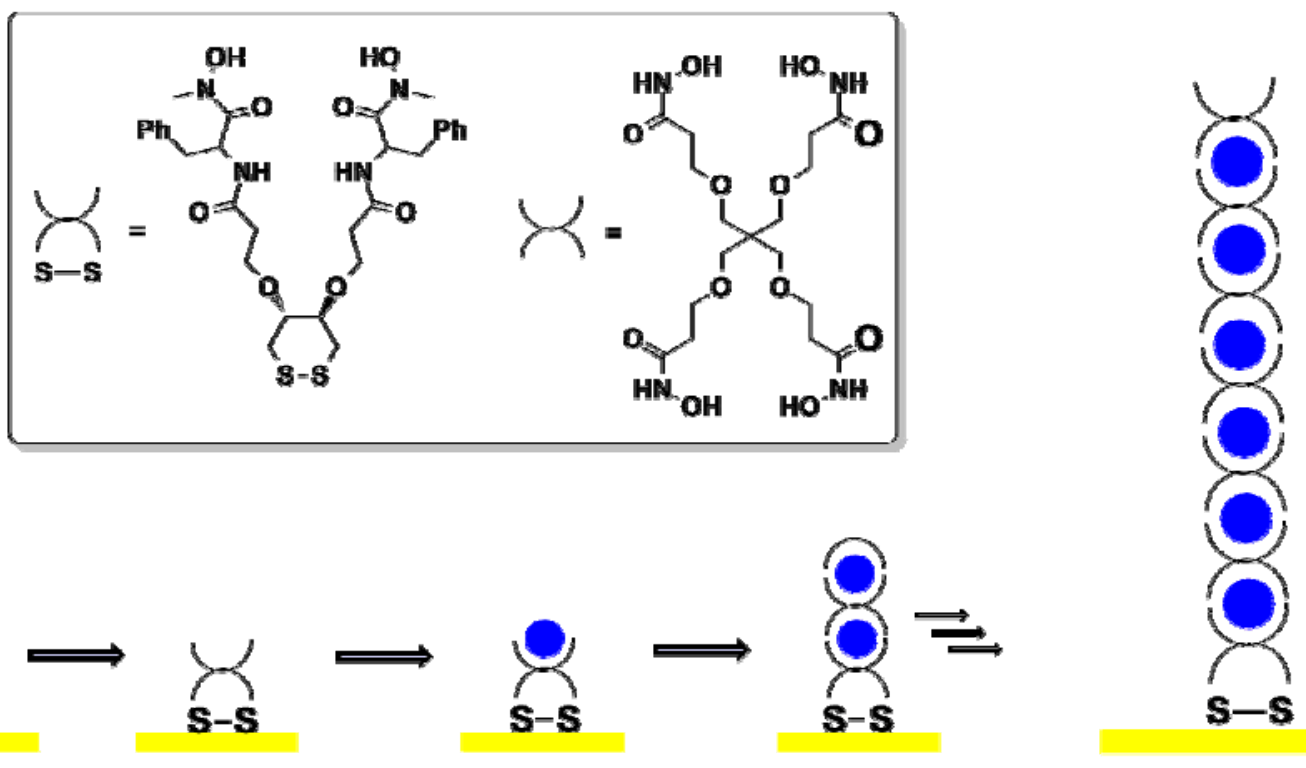

$=$ Metal $\left(\mathrm{Zr}^{4+}, \mathrm{Ce}^{4+}, \mathrm{Ti}^{4+}\right)$

Figure 2.7 Schematic presentation of the molecules used for multilayer construction and an idealized structure of the coordination based multilayers. Adapted with permission from ref 17. Copyright 2004 American Chemical Society. 
Mallouk and co-workers used similar type tetravalent- $\left(\mathrm{Zr}^{4+}, \mathrm{Hf}^{4+}\right)$ or divalent $\left(\mathrm{Zn}^{2+}\right.$, $\mathrm{Cu}^{2+}$ ) metal ions and phosphonates as a ligand to build-up multilayers in a supramolecular metal-ligand coordination manner. ${ }^{[18]}$ Papadimitrakopoulos et al. demonstrated the stepwise self-assembly process of diethyl zinc and bisquinoline on a silicon substrate resulting in films capable of electroluminescence. Assembled films also showed a high refractive index and uniformity. ${ }^{[19]}$ McGimpsey and co-workers developed a non-covalent metal ligand coordination for the assembly of supramolecular photocurrent-generating systems. ${ }^{[20]}$ In their system, the light absorbing group (pyrene) was noncovalently coupled to a gold surface via metal-ligand complexation. These systems were noncovalently assembled by sequential deposition of three or more components, showing high stability and high current generation on gold surface.

\subsubsection{Assembly of nanoparticles on SAMs}

Murray at al. developed a new way to fabricate monolayer or multilayer films of carboxylate-functionalized gold nanoparticles onto a mercaptoundecanoic acid monolayer. ${ }^{[21]}$ Nanoparticles were attached via divalent metal ions $\left(\mathrm{Cu}^{2+}, \mathrm{Zn}^{2+}, \mathrm{Pb}^{2+}\right)$. Attachment of additional layers of particles was performed by repeated dipping cycles of metal ions and particles, resulting in the formation of network nanoparticle films. The group of Rubinstein reported gold nanoparticle mono- and multilayers on gold surfaces using coordination chemistry. ${ }^{[22]} \mathrm{Au}$ nanoparticles capped with a monolayer of 6-mercaptohexanol, were modified by partial substitution of bishydroxamic acid disulfide ligand molecules into their capping layer. A monolayer of the ligand-modified Au nanoparticles was assembled via coordination with $\mathrm{Zr}^{4+}$ ions onto a gold substrate precoated with a self-assembled monolayer of the bishydroxamate disulfide ligand. Layer-by-layer construction of nanoparticle 
multilayers was achieved by alternate binding of $\mathrm{Zr}^{4+}$ ions and ligand-modified nanoparticles onto the first nanoparticle layer (Figure 2.8).

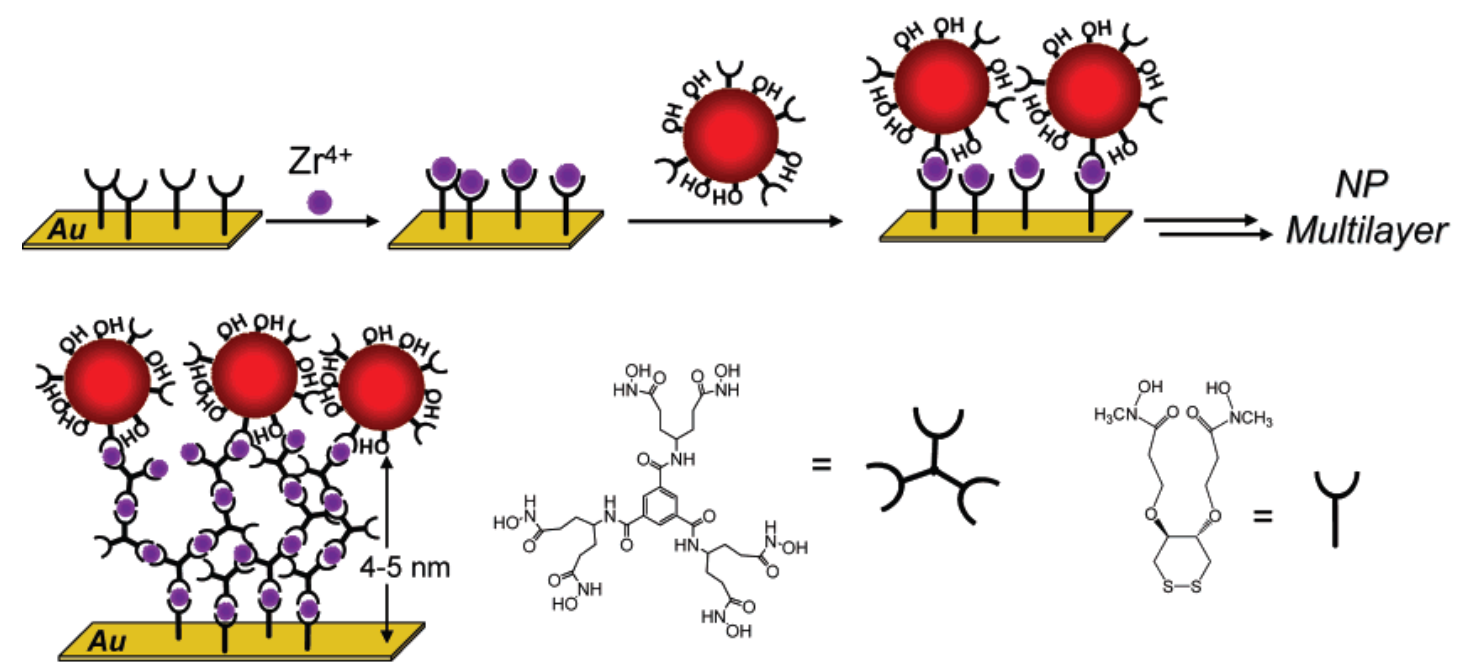

Figure 2.8 Stepwise assembly of bishydroxamate-bearing Au nanoparticle multilayers onto bishydroxamate disulfide SAMs on a gold surface via $\mathrm{Zr}^{4+}$ ions (top). Controlled spacing of nanoparticles from the gold surface using a hexahydroxamate ligand (bottom). Reproduced with permission from ref 22. Copyright 2005 American Chemical Society.

Chen and co-workers used metal-ligand coordination (divalent metal ions and pyridine moieties as a ligand) for the assembly of nanoparticles on surfaces. ${ }^{[23]}$ The thickness of the nanoparticle layers was controlled by repetitive alternate dipping cycles (Figure 2.9). 
<smiles>CC(C)C=C=CCS</smiles>
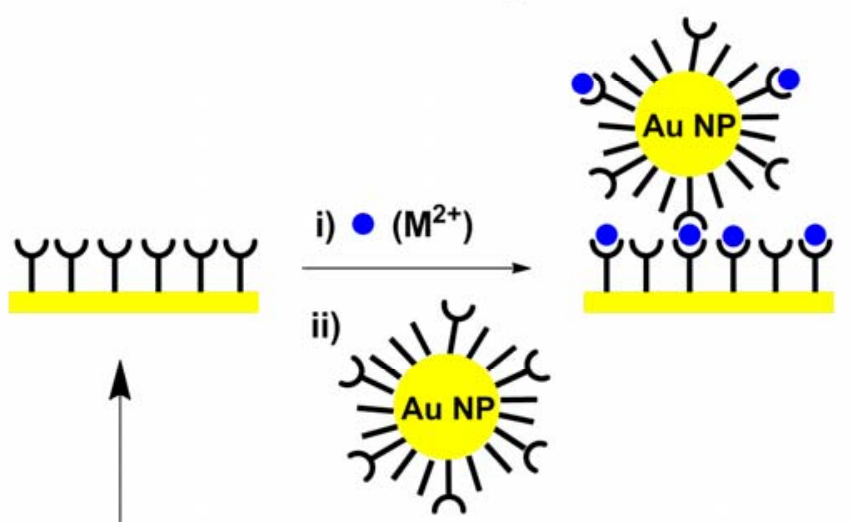

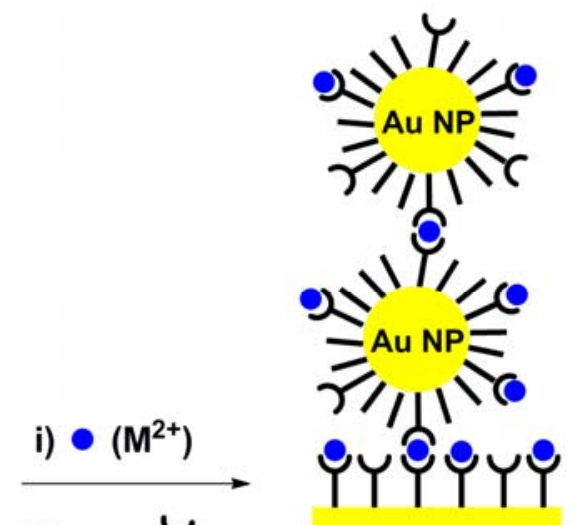

ii)

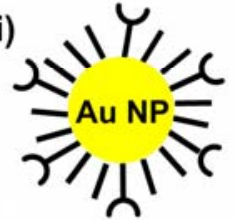

EDTA

Figure 2.9 Procedure for nanoparticle assembly by the chelating interactions between divalent (transition) metal ions and pyridine moieties. Adapted with permission from ref 23. Copyright 2002 American Chemical Society.

Reinhoudt et al. used oordination chemistry to grow isolated nanoparticles on surfaces. ${ }^{[24]} \mathrm{Pd}^{2+}$ containing pincer adsorbate molecules were embedded into mercaptoundecanol and decanethiol SAMs on gold. Monolayer-protected Au nanoclusters bearing phosphine moieties at the periphery were coordinated to SAMs containing individual $\mathrm{Pd}^{2+}$ pincer molecules via supramolecular metal-ligand interactions. 


\subsection{Assembly by electrostatic interactions}

One of the most simple and versatile methods for the assembly of $2 \mathrm{D}$ and $3 \mathrm{D}$ structures is electrostatic self-assembly. The driving force for the assembly is the ionic interaction between oppositely charged entities (polymers, nanoparticles, and substrates), providing the fabrication of functional mono- or multilayer architectures in a stepwise fashion. Electrostatic self-assembly has been the most widely used method for the assembly of the different materials on surfaces. Electrostatic forces are strong enough to create stable assemblies, but weak enough to respond to environmental changes such as variations of ionic strength or $\mathrm{pH}$.

\subsubsection{Assembly of molecules on SAMs}

The group of Reinhoudt used electrostatic self interactions to prepare SAMs of organic radicals on silicon substrates. ${ }^{[25]}$ For this purpose, amino groups on surface were protonated by rinsing with a 4-morpholineethanesulfonic acid monohydrate buffer ( $\mathrm{pH}$ 5.6) to give a positively charged surface and, subsequently, the substrate was immersed in a solution of 4-carboxytetradecachlorotriphenylmethyl radical $(\mathrm{PTMCOOH})$ to give a SAM of the organic radical (Figure 2.10). Calvo and co-workers reported the assembly of some enzymes such as glucose and lactate oxidases on gold by electrostatic adsorption. ${ }^{[26]}$ A polycation, poly(allylamine), was assembled onto a gold electrode modified with 3-mercapto-propanesulfonic acid by electrostatic interaction. Enzymes were also immobilized onto the polycation layer electrostatically. 


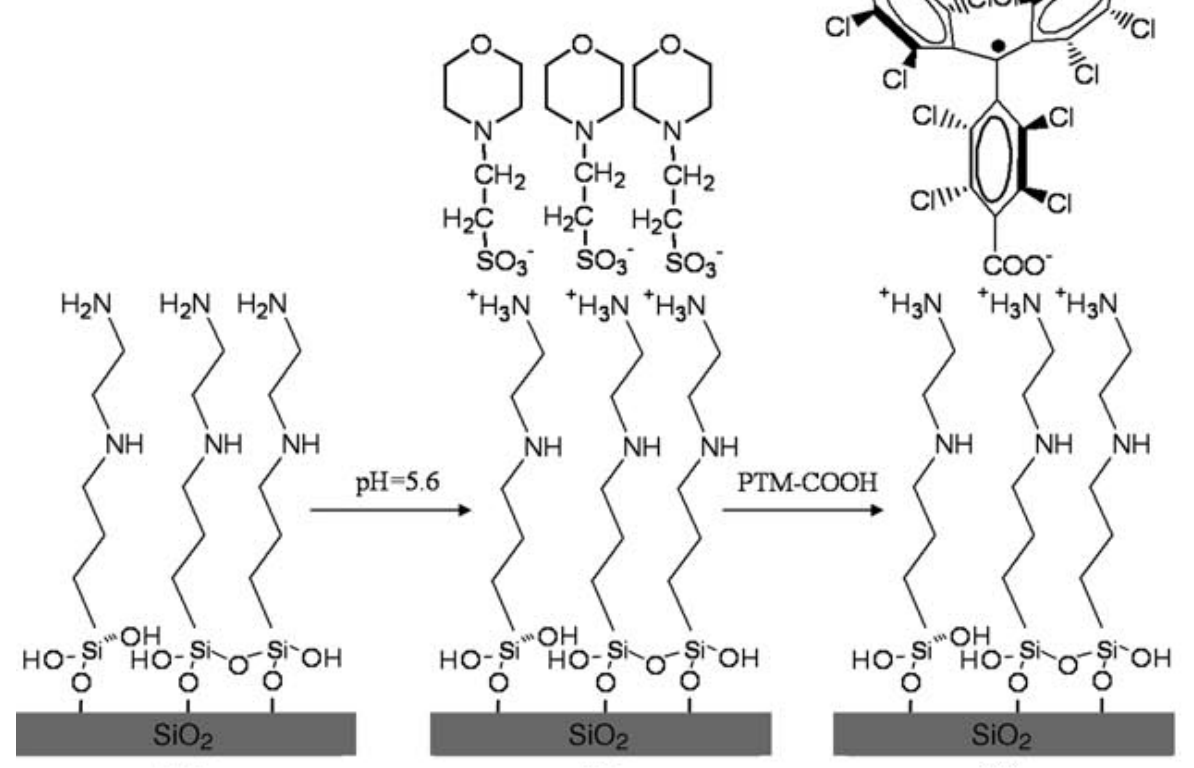

Figure 2.10 Formation of the polychorotriphenylmethyl (PTM) radical $\mathrm{SAMs}$ on a $\mathrm{SiO}_{2}$ surface by electrostatic interaction. Reproduced with permission from ref 25. Copyright 2007 Wiley-VCH Verlag GmbH \& Co. KGaA.

An approach for the construction of photoactive devices is highly ordered immobilization of photofunctional molecules on surfaces. For that purpose, Tamiaki et al. reported the electrostatic layer-by-layer adsorption of the light harvesting complexes (chlorosomes) from the green sulfur photosynthetic bacterium Chlorobium (Chl.) tepidum onto a glass substrate using the cationic linear polymer polylysine. ${ }^{[27]}$ Burgin and co-workers examined the electrostatic nature of single walled carbon nanotubes (SWNTs) adsorption on amine surfaces via electrostatic interactions where both the amine and the SWNTs were treated by various $\mathrm{pH}$ buffers prior to solution deposition of nanotubes. ${ }^{[28]}$ In a similar manner, Bao et al. fabricated amine silane SAMs with varying end groups that led to adsorption of submonolayer nanotube network films with varying degrees of alignment and density. ${ }^{[29]}$ The protonation of amine-coated surfaces influences this adsorption and chirality sorting of SWNTs (Figure 2.11). 

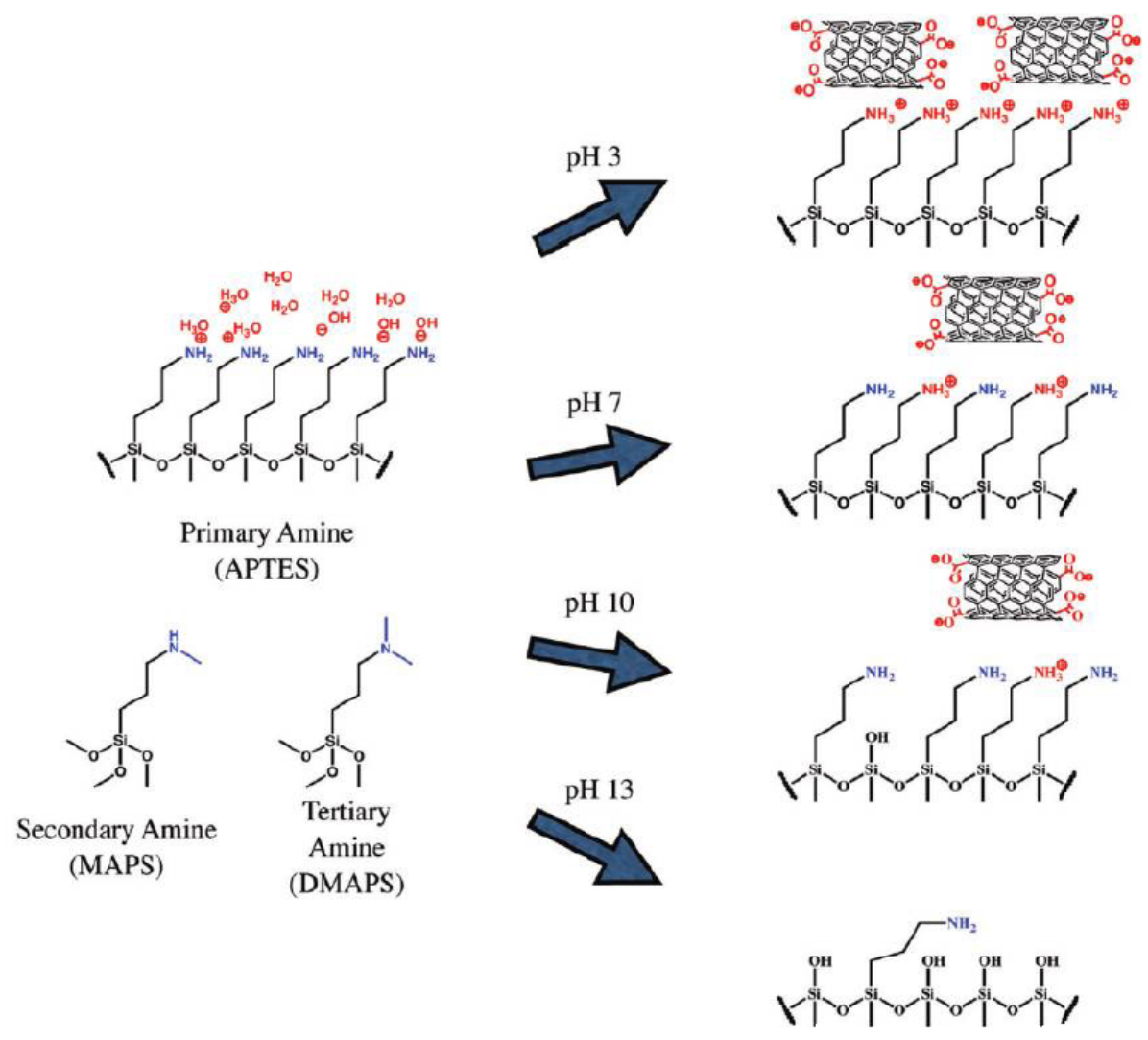

Figure 2.11 Schematic illustration of influence of protonation on SWNTs adsorption. Reproduced with permission from ref 29. Copyright 2010 American Chemical Society.

\subsubsection{Assembly of nanoparticles on SAMs}

Ionic interactions have also been used for nanoparticle assembly on surfaces. For instance, Auer and co-workers studied the assembly of gold nanoparticles on planar gold surfaces precoated with mercaptohexadecanoic acid using bisbenzamidines as a linker between negatively charged gold nanoparticles and the surface. ${ }^{[30]}$ Murphy and co-workers used gold nanorods to assemble on a surface by electrostatic interaction. ${ }^{[31]}$ Gold nanorods were stabilized with cetyltrimethylammonium bromide (CTAB) and assembled on a gold surface modified with 16-mercaptohexadecanoic acid. Attractive electrostatic interactions 
between the carboxylic acid group on the SAM and the positively charged CTAB molecules are likely responsible for the nanorod immobilization (Figure 2.12).

Gold coated substrate

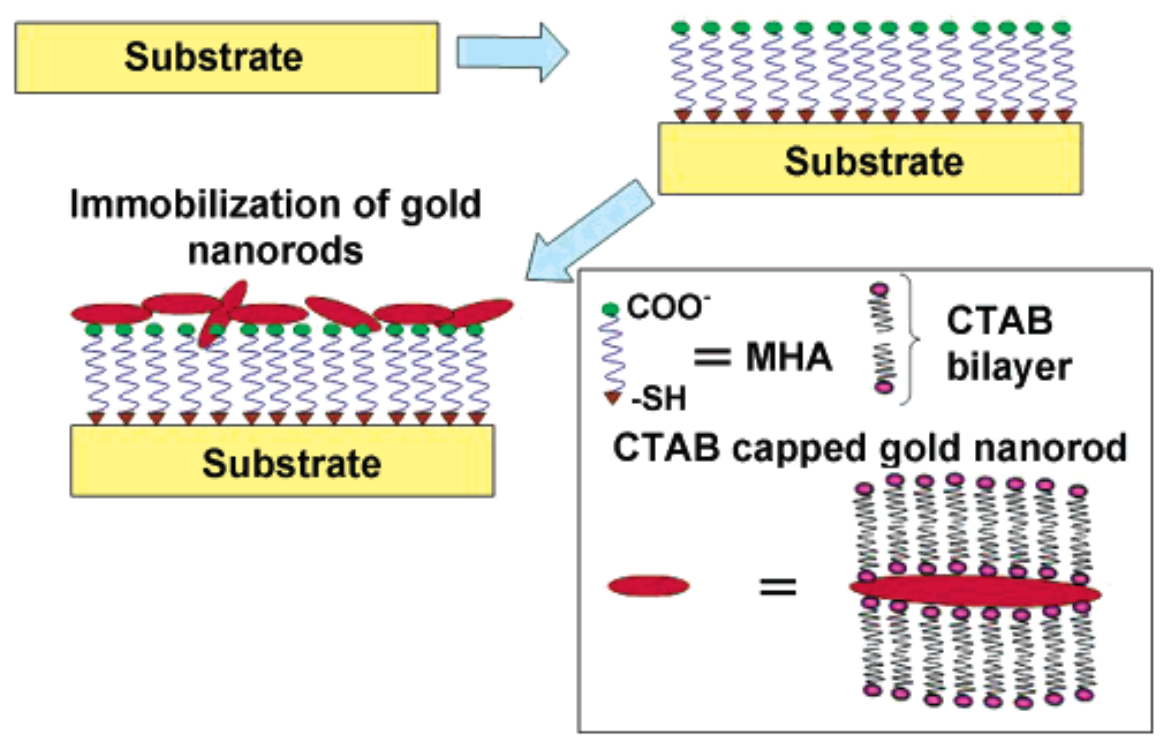

Figure 2.12 Schematic immobilization of CTAB-Modified Gold Nanorods onto SAMs of 16MHA. Reproduced with permission from ref 31. Copyright 2004 American Chemical Society.

A similar approach was used by Sastry and co-workers. ${ }^{[32]}$ They described the formation of self-assembled monolayers (SAMs) of an aromatic bifunctional molecule, 4-aminothiophenol (4-ATP) on gold and the subsequent organization of carboxylic acid derivatized silver colloidal particles.

The controlled organization and precise positioning of nanoparticles on 2D surfaces are essential for the development of new functional materials. Some studies focused on the combination of top-down surface patterning with self-assembly of particles via electrostatic 
interactions. For example, patterning by photolithography, ${ }^{[33]}$ soft lithography, ${ }^{[34]}$ nanoimprint lithography, ${ }^{[35]}$ and scanning probe lithography ${ }^{[36]}$ have been widely used for the fabrication of electrostatically assembled nanoparticles on patterned surfaces.

\subsection{Assembly by host-guest interactions}

Host-guest chemistry plays an important role in the construction of supramolecular architectures on surfaces. Calixarenes, cucurbiturils (CB), and cyclodextrins (CD) are interesting host molecules which form stable and specific inclusion complexes with a variety of organic guest molecules. Monolayers of these host molecules on surfaces constitute the unique platforms for the immobilization of various guest molecules in a multivalent supramolecular fashion. This section describes the supramolecular assembly onto different receptor surfaces by host-guest interactions.

\subsubsection{Assembly of molecules on SAMs}

Calixarene monolayers have been synthesized and charaterized extensively by the group of Reinhoudt. ${ }^{[37]}$ Calixarenes formed well-packed and ordered monolayers capable of interacting with different guest molecules in aqueous solution. Gupta and co-workers showed that calix[4]arene monolayers could discriminate between two structural isomers of hydroxybutyrolactone by surface immobilization of the receptor units. ${ }^{[38]}$

Monolayers of cucurbit[6]uril, a macrocyclic cavitand comprising six glycoluril units, have been described by Kim and co-workers. ${ }^{[39]}$ Alkene functionalized CB[6] was reacted with surface immobilized thiols under UV light, resulting in $\mathrm{CB}[6]$ monolayers on a glass surface. The $\mathrm{CB}[6]$ modified glass recognizes small molecules such as spermine which is known to form a stable host-guest complex with $\mathrm{CB}[6]$ (Figure 2.13). 


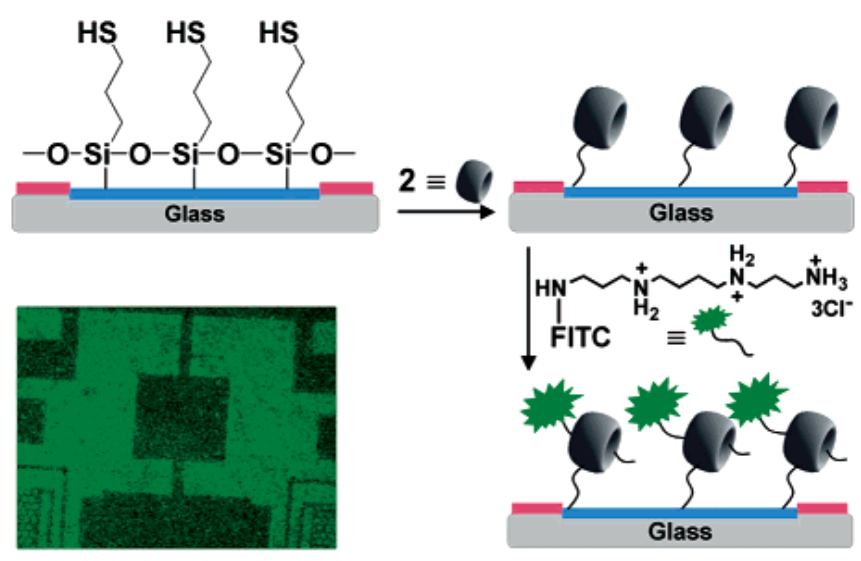

Figure 2.13 Cartoon for anchoring a $\mathrm{CB}[6]$ derivative onto a patterned glass and detection of fluorescently labeled spermine by the $\mathrm{CB}[6]$ modified surface. Reproduced with permission from ref 39. Copyright 2003 American Chemical Society.

Zhang et al. described a general protocol based on the spontaneous adsorption of cucurbit[n]uril $(\mathrm{CB}[\mathrm{n}])$ molecules through a strong multivalent interaction between $\mathrm{CB}[\mathrm{n}]$ and gold ${ }^{[40]}$ Their method does not require any prior modification or special treatment of $\mathrm{CB}[\mathrm{n}]$ molecules, and is applicable for all members of the $\mathrm{CB}[\mathrm{n}]$ family, at least $\mathrm{CB}[6-8]$ (Figure 2.14).

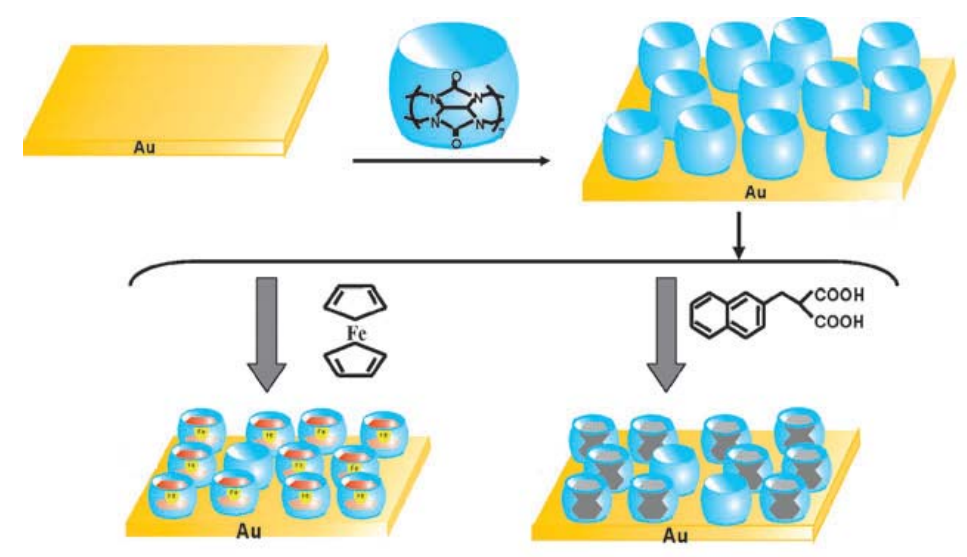

Figure 2.14 Schematic illustration of the construction of a self-assembled $\mathrm{CB}[\mathrm{n}]$ monolayer on a gold surface and the formation of inclusion complexes. Reproduced with permission from ref 40. Copyright 2008 The Royal Society of Chemistry. 
Jonkheijm and co-workers also used a $\mathrm{CB}$ [7] monolayer for the immobilization of the proteins through a monovalent supramolecular interaction. ${ }^{[41]}$ Their technique allows printing of stable protein monolayers in well-defined formats to be achieved with controlled protein orientation and with subsequent replacement of the protein monolayer by a small synthetic ligand (Figure 2.15).

Monolayers of cyclodextrin (CD SAMs) have been studied and extensively characterized. The immobilization of a wide range of (bio)molecules functionalized with multiple guest units onto CD SAMs on gold and silicon oxide surfaces have been reported by different research groups (Figure 2.16). ${ }^{[42]}$ Huskens and Reinhoudt et al. introduced the concept of "molecular printboards", for the stable positioning and assembly of guest functionalized dendrimers, ${ }^{[43]}$ nanoparticles, ${ }^{[4]}$ proteins, ${ }^{[45]}$ and fluorescent small molecules ${ }^{[46]}$ onto CD SAMs. Molecular patterns of (bio)molecules have also been prepared on these molecular printboards by using lithographic techniques such as microcontact printing and dip-pen nanolithography. ${ }^{[35,47]}$

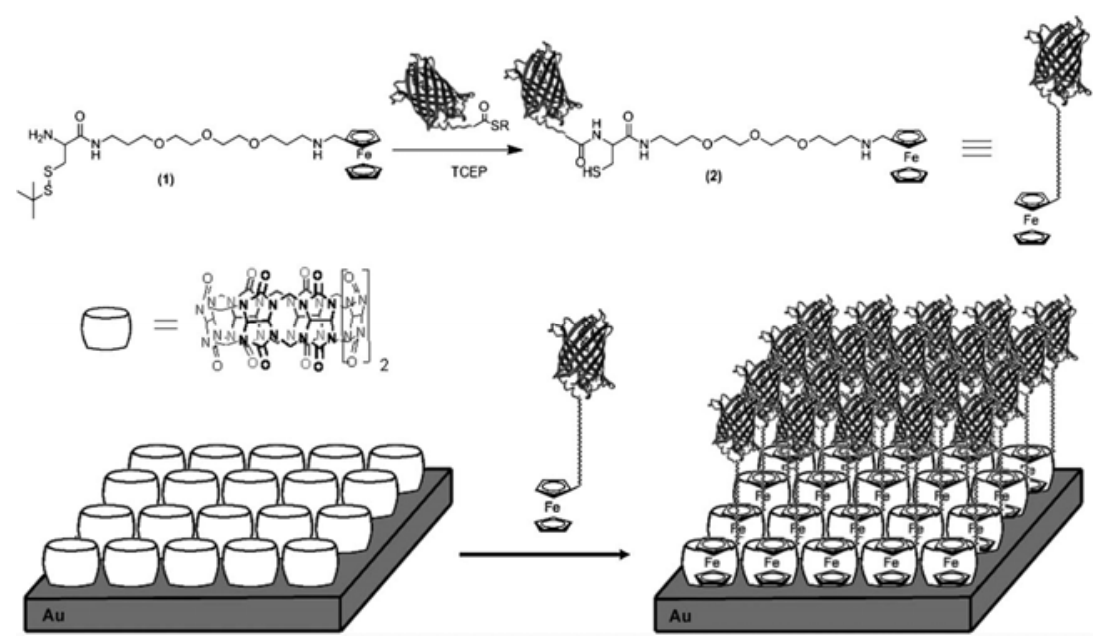

Figure 2.15 Ligation of a ferrocene-cysteine derivative (1) with yellow fluorescent protein (YFP) and immobilization of the resulting ferrocene-YFP (2) onto a $\mathrm{CB}[7]$ monolayer. Reproduced with permission from ref 41. Copyright 2010 Wiley-VCH Verlag GmbH \& Co. $\mathrm{KGaA}$. 

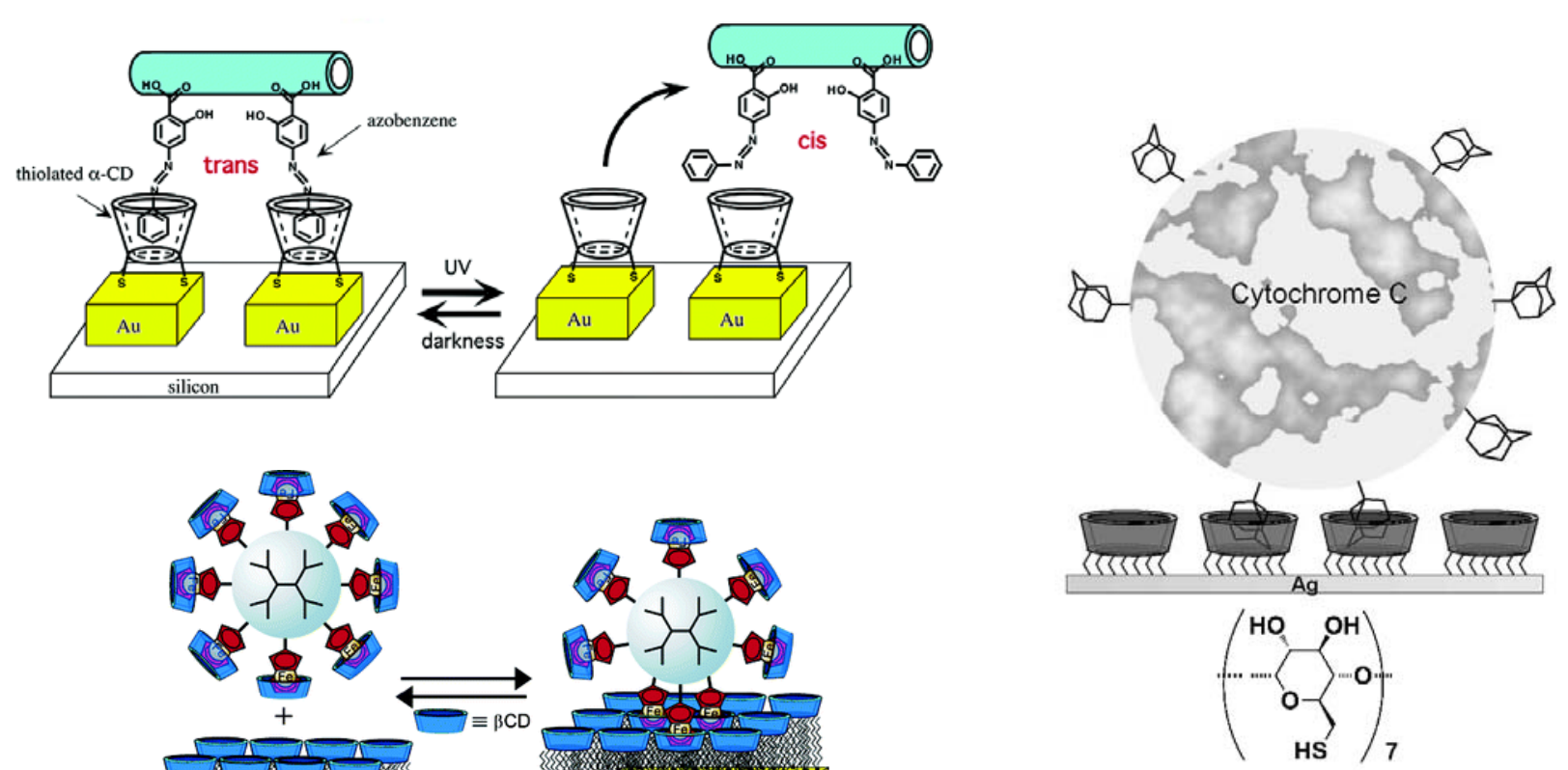

Figure 2.16 Supramolecular interaction motifs at CD SAMs. Reproduced with permission from ref 42b (Copyright 2003 American Chemical Society), ref 42d (Copyright 2002 American Chemical Society), ref 42e (Copyright 2004 American Chemical Society).

\subsection{Combination of different orthogonal supramolecular interaction motifs}

All supramolecular interactions reviewed here, i.e. hydrogen bonding, metal-ligand coordination, electrostatic, and host-guest interactions, have in common a high level of structural definition and tunable strength, which allow the design of functional materials at the molecular level. As discussed above, these weak interactions were employed individually to build functional supramolecular architectures on surfaces. The combination of different orthogonal supramolecular interaction motifs is essential for the fabrication of complex hybrid organic-inorganic materials and stimuli-responsive surfaces. This section highlights the recent developments of the combination of different orthogonal interaction motifs to yield hierarchical supramolecular assemblies on surfaces. 
Huskens et al. have described the combination of different orthogonal supramolecular systems on molecular printboards. In a first study, they demonstrated the immobilization of a supramolecular capsule at the surface. ${ }^{[4]}$ Two different orthogonal systems, host-guest and electrostatic interactions, were utilized to generate a capsule on a surface. To build such a supramolecular capsule, they used noncovalent attachment of one component of the molecular capsule on the CD SAM via orthogonal host-guest interaction followed by the self-assembly of the second component at the interface through ionic interaction (Figure 2.17A). Another study describes the multivalent binding of a supramolecular complex at a multivalent host surface by combining the orthogonal CD hostguest and metal ion-ethylenediamine coordination motifs. ${ }^{[49]}$ In this orthogonal supramolecular system, a heterotropic divalent linker, with a CD-complexing adamantyl (Ad) group and an M(II)-complexing ethylenediamine ligand is employed. This allows the linker to bind to $\mathrm{CD}$ in solution as well as to $\mathrm{CD}$ immobilized at SAMs (Figure 2.17B). A similar study describes the preparation of vesicles bearing host units (cyclodextrin) and their interactions with guest (adamantyl) functionalized ligands via orthogonal multivalent hostguest and metal-ligand complexation. ${ }^{[50]}$ Vesicles of amphiphilic cyclodextrin recognized metal coordination complexes with adamantyl ligands via inclusion in the host cavities at the vesicle surface. In the case of divalent $\mathrm{Cu}$ (II) complexes, the interaction was predominantly intravesicular. In the case of $\mathrm{Ni}(\mathrm{II})$, the interaction was effectively intervesicular, and addition of the guest-metal complex resulted in aggregation of the vesicles into dense, multilamellar clusters. The valency of molecular recognition at the surface of vesicles and the balance between intravesicular and intervesicular interaction could be tuned by metal coordination of guest molecules. Another study, they presented the attachment of streptavidin (SAv) to CD SAM via orthogonal host-guest and SAv-biotin interactions. ${ }^{[51]}$ The orthogonal linkers consist of a biotin functionality for binding to SAv and adamantyl functionalities for 
host-guest interactions at CD SAM. The approach was used for build-up and patterning of protein nanostructures at interfaces using a sequence of host-guest and SAv-biotin interaction.
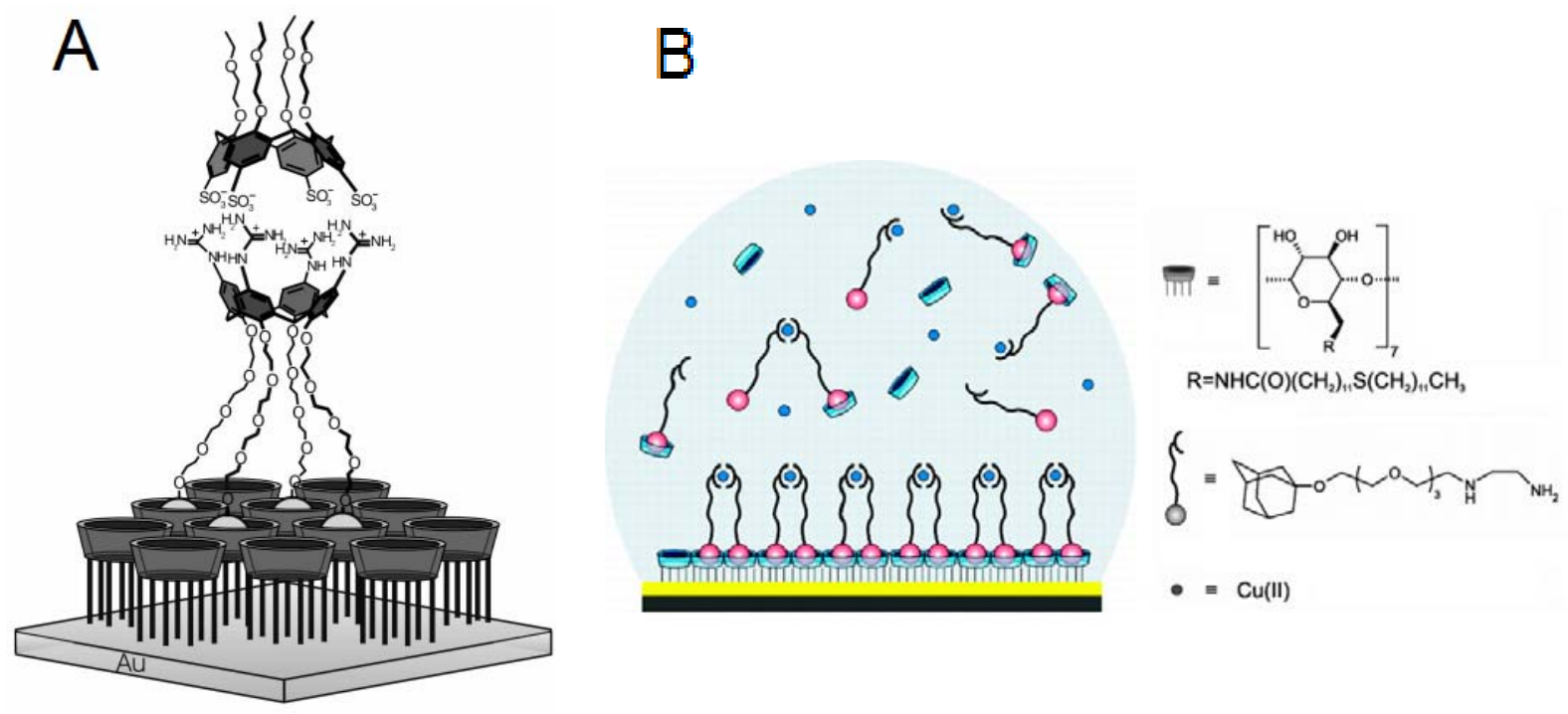

Figure 2.17 A) Formation of molecular capsule at CD SAMs via host-guest and electrostatic interactions. Reproduced with permission from ref 48. Copyright 2004 American Chemical Society. B) Complex formation on CD SAMs by host-guest and metal-ligand coordination. Reproduced with permission from ref 49. Copyright 2006 American Chemical Society.

The encapsulation of anionic dyes in immobilized dendrimers has been described to occur via orthogonal multivalent host-guest and electrostatic interactions. ${ }^{[52]}$ Fifth-generation poly(propylene imine) dendrimers, modified with 64 apolar adamantyl groups, have been immobilized on cyclodextrin host monolayers on glass by supramolecular microcontact printing. The immobilized dendrimers retained their guest binding properties and functioned as "molecular boxes" that can be filled with fluorescent dye molecules from solution (Figure 2.18). 


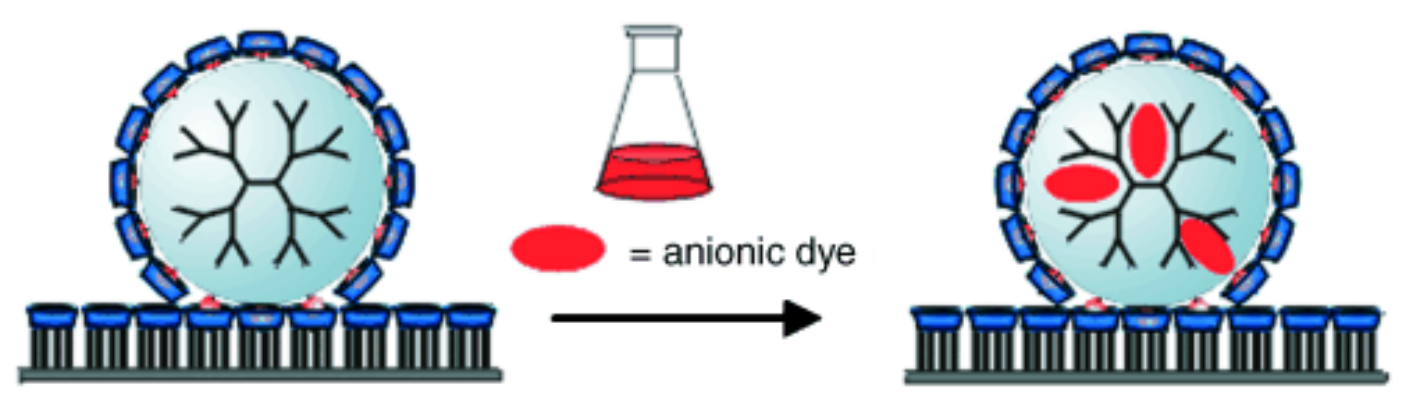

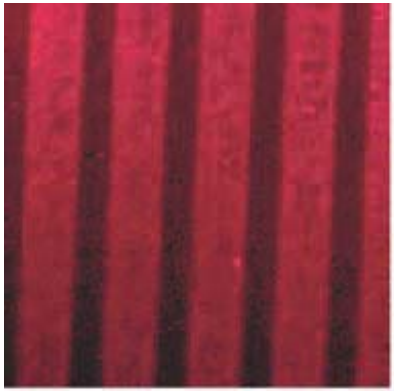

Bengal Rose

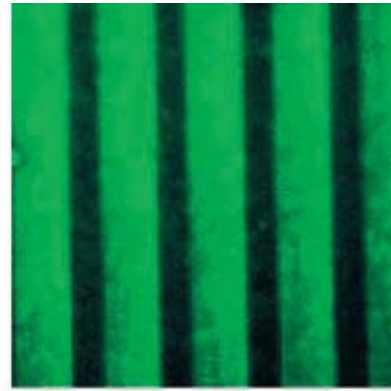

Fluorescein

Figure 2.18 Schematic representation of the filling of immobilized dendrimer patterns with anionic dyes (upper). Confocal microscopy images after microcontact printing of dendrimer on a molecular printboard, followed by filling of the immobilized dendrimers with Bengal Rose and fluorescein dyes (lower). Reproduced with permission from ref 52. Copyright 2005 Wiley-VCH Verlag GmbH \& Co. KGaA.

The versatility and advantages of the molecular printboard for attaching proteins, for example, controllable binding constants and the suppression of nonspecific interactions, were combined with His-tagged proteins via host-guest and metal-ligand interactions. ${ }^{[53]}$ His $_{6^{-}}$ tagged proteins have been attached to a molecular printboard in a selective manner by using the supramolecular blocking agent $\mathbf{3}$ and $\mathrm{Ni} \cdot \mathbf{4}$ as depicted in Figure 2.19. 


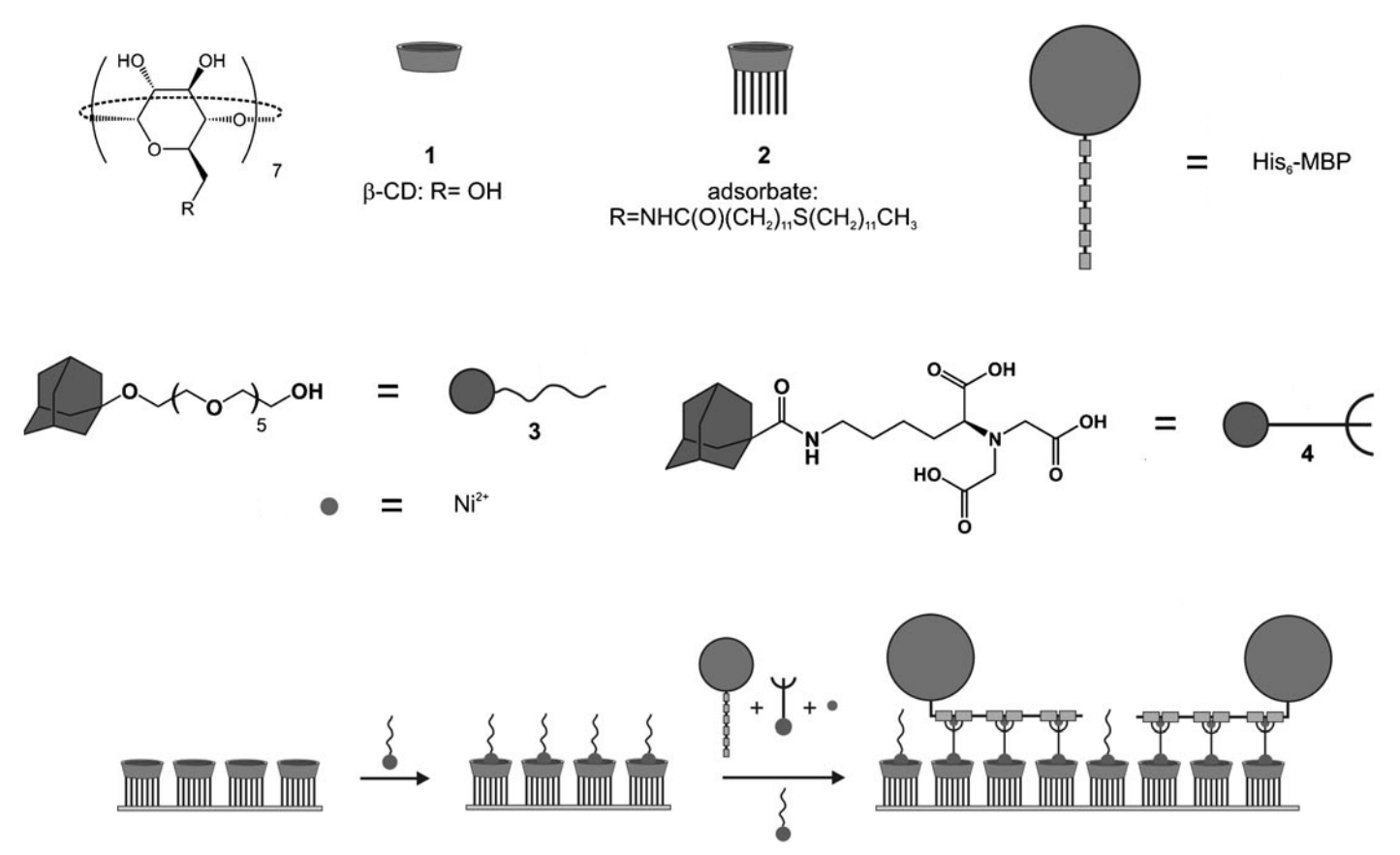

Figure 2.19 Structures of compounds: $\beta$-cyclodextrin 1, adsorbate for SAMs on gold 2, adamantyl linkers 3 and $\mathbf{4}$, nickel, $\mathrm{His}_{6}-\mathrm{MBP}$ and cartoon for the binding of $\mathrm{His}_{6}-\mathrm{MBP}$ through $\mathrm{Ni} \cdot \mathbf{4}$ to $\mathrm{CD} \mathrm{SAMs}$, in competition with monovalent blocking agent 3 . Reproduced with permission from ref 53. Copyright 2008 Wiley-VCH Verlag GmbH \& Co. KGaA.

Another contribution describes the patterning of silica substrates with thymine as hydrogen bonding unit and positively charged $N$-methylpyridinium containing polymers using photolithography, and the subsequent orthogonal supramolecular modification of these surfaces using diaminopyridine- functionalized polystyrene and carboxylate-derivatized $\mathrm{CdSe} / \mathrm{ZnS}$ core-shell nanoparticles through the combination of diaminopyridine-thymine hydrogen bonding and pyridinium- carboxylate electrostatic interactions (Figure 2.20). ${ }^{[54]}$ 

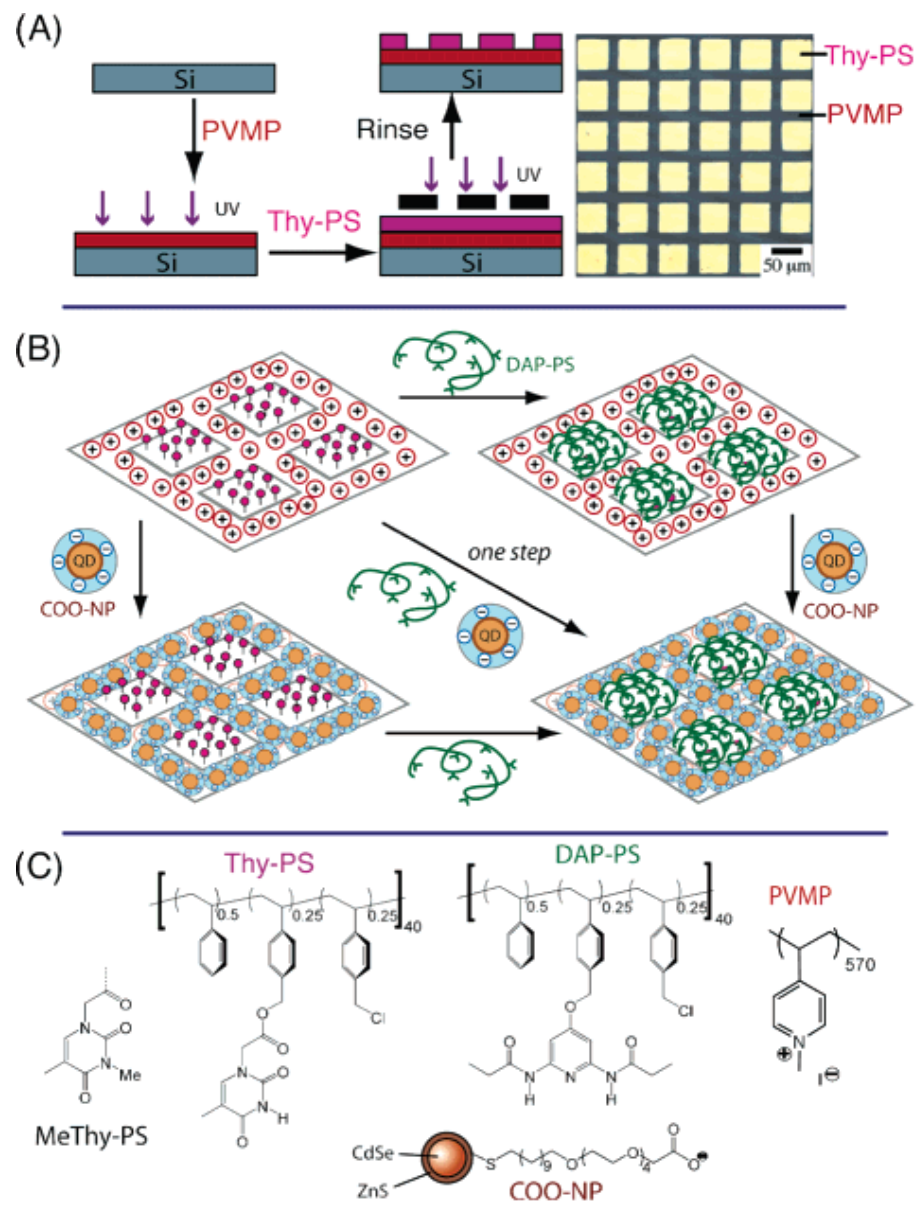

Figure 2.20 Schematic illustration of the fabrication process. (A) Formation of the patterned PVMP/Thy-PS surface and optical micrograph of the resulting pattern. (B) One-step and sequential orthogonal functionalization by DAP-PS and COO-NP through PS-Thy:PS-DAP recognition and PVMP:COO-NP electrostatic interactions. (C) Chemical structures of the materials, including control polymer MeThy-PS. Reproduced with permission from ref 54 . Copyright 2006 American Chemical Society.

The group of Haga developed DNA nanowires via orthogonal self-assembly by assistance of a SAM on the surface. ${ }^{[55]}$ Orthogonal self-assembly was applied to the surface for the selective modification of the DNA capture molecules on the Au electrode. Two anchor groups of thiol and phosphonic acid were used to discriminate between $\mathrm{Au}$ and $\mathrm{SiO}_{2}$, since a 
thiol group selectively attaches to the $\mathrm{Au}$ surface and a phosphonate group attaches to the $\mathrm{SiO}_{2}$ surface. Once the DNA trapping molecule is selectively attached to gold patterns on silicon substrate, DNA is captured from solution and used as a nanowire between two gold patterns (Figure 2.21).
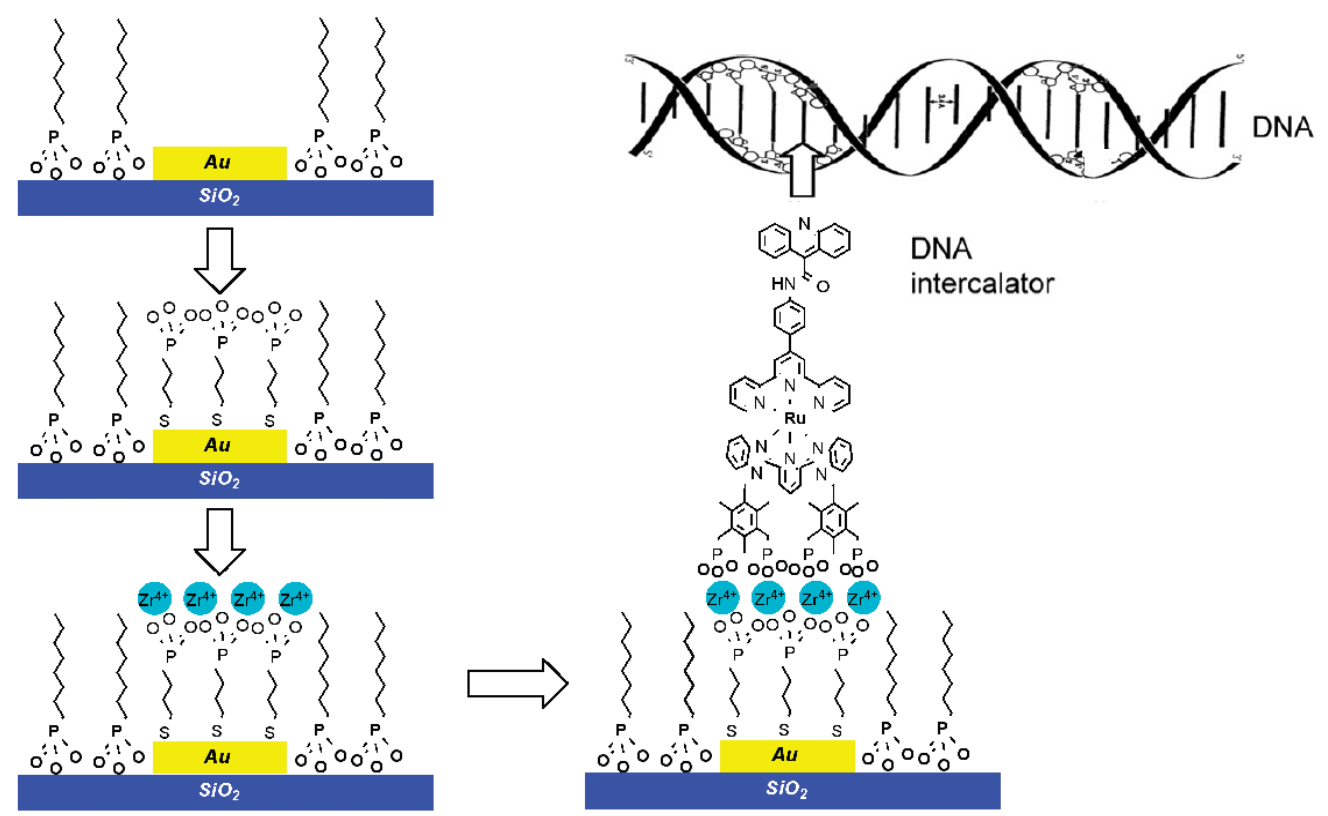

Figure 2.21 Schematic illustration of surface modification for DNA capture by metal coordination directed orthogonal assembly on gold patterned silicon. Reproduced with permission from ref 55. Copyright 2008 American Chemical Society.

An example of the combination of electrostatic interaction with $\pi$ - $\pi$ stacking on a surface has been reported by Shinkai and co-workers. ${ }^{[56]}$ They used a hexacationic homooxacalix[3]arene-[60]fullerene 2:1 complex to make a monolayer or a monolayer-like ultra-thin film on an anion-coated gold surface. They also studied the photoelectrochemical response of the monolayers under UV-irradiation (Figure 2.22). 


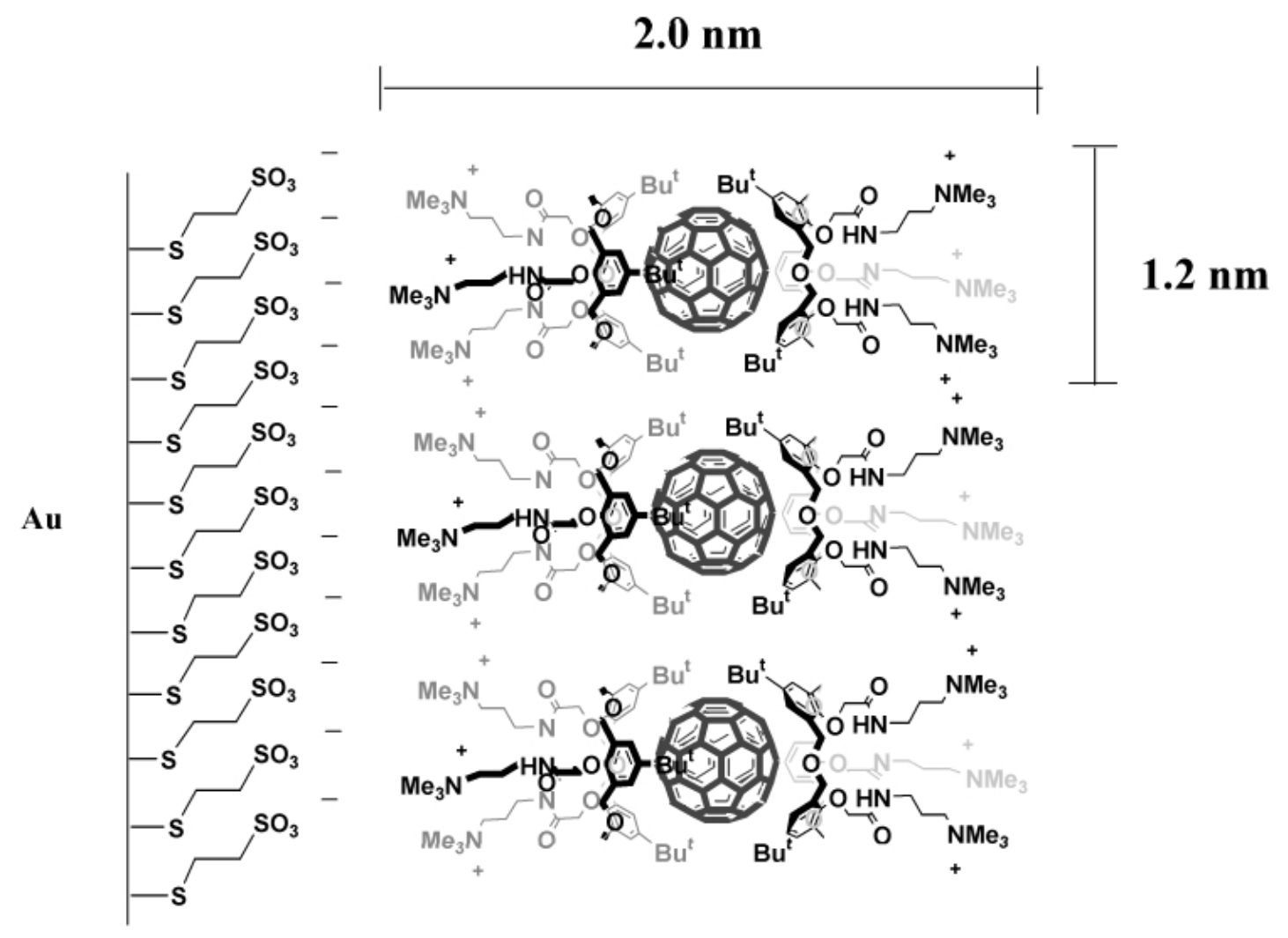

Figure 2.22 Adsorption of sodium 2-mercaptoethanesulfonate $\left(1^{\text {st }}\right.$ layer $)$ and $1-[60]$ fullerene ( $2^{\text {nd }}$ layer) on a gold surface. Reproduced with permission from ref 56. Copyright 2000 The Royal Society of Chemistry.

Tait et al. developed the concept of stabilizing and ordering 1D coordination structures at a surface ${ }^{[57]}$ Hydrogen bonding interactions with the second molecular species improved the stability and ordering of the copper-pyridyl 1D coordination chains. This combination of the selective orthogonal interactions allowed the fine-tuning of the supramolecular system by choice of the building blocks. In the group of Dalcalane, hierarchical assembly on silicon using host-guest and hydrogen bonding interactions was developed. ${ }^{[58]}$ The multistep growth of supramolecular structures on the surface resulted from the combined use of orthogonal host-guest and hydrogen bonding interactions. Using this strategy, hybrid and multifunctional 
materials could be constructed. Fasel et al. reported the two-dimensional mono- and bicomponent self-assembly of three closely related diaminotriazine-based molecular building blocks and a complementary perylenetetracarboxylic diimide with the interplay of hydrogen bonding, dipolar interactions, and metal coordination. ${ }^{[59]}$ They showed that the simplest molecular species, bis-diaminotriazine-benzene, only interacts via hydrogen bonds and forms a unique supramolecular pattern on a gold surface. For the two related molecular species, which exhibit in addition to hydrogen bonding also dipolar interactions and metal coordination, the number of distinct supramolecular structures increases dramatically with the number of possible hierarchical assemblies with orthogonal interactions.

\subsection{Conclusions}

The use of supramolecular chemistry and molecular self-assembly including hydrogen bonding, metal coordination, electrostatic and host-guest interactions to direct the immobilization of functional systems on surfaces have attracted considerable attention in modern research due to their special characteristic features such as high specificity, controlled affinity and reversibility. In this chapter some examples of orthogonal supramolecular interactions for the construction of functional materials with tunable properties on flat surfaces have been reviewed. Although these noncovalent interactions were used in many studies individually to build supramolecular architectures on surfaces, there are only limited numbers of examples that address the combination of different supramolecular interactions for the generation of functional monolayers. Hence, the development of hierarchical assemblies by using the combination of different noncovalent interactions still requires more efforts to allow the fabrication of functional surfaces. In this thesis, the concept of orthogonal supramolecular assembly is employed to form functional monolayers that are promising in sensor applications. 


\subsection{References}

[1] J.-M. Lehn, Supramolecular Chemistry, Concepts and Perspectives, VCH, Weinheim, Germany, 1995.

[2] a) T. J. Gardner, C. D. Frisbie, M. S. Wrighton, J. Am. Chem. Soc. 1995, 117, 69276933; b) J. J. Hickman, P. E. Laibinis, D. I. Auerbach, C. F. Zou, T. J. Gardner, G. M. Whitesides, M. S. Wrighton, Langmuir 1992, 8, 357-359; c) P. E. Laibinis, J. J. Hickman, M. S. Wrighton, G. M. Whitesides, Science 1989, 245, 845-847; d) J. M. Pollino, M. Weck, Chem. Soc. Rev. 2005, 34, 193-207; e) J. Tien, A. Terfort, G. M. Whitesides, Langmuir 1997, 13, 5349-5355.

[3] a) J. A. A. W. Elemans, A. E. Rowan, R. J. M. Nolte, J. Mater. Chem. 2003, 13, 2661-2670; b) H. Hofmeier, U. S. Schubert, Chem, Commun, 2005, 2423-2432.

[4] G. M. Credo, A. K. Boal, K. Das, T. H. Galow, V. M. Rotello, D. L. Feldheim, C. B. Gorman, J. Am. Chem. Soc. 2002, 124, 9036-9037.

[5] T. B. Norsten, E. Jeoung, R. J. Thibault, V. M. Rotello, Langmuir 2003, 19, 70897093.

[6] G. Cooke, J. Couet, J. F. Garety, C. Q. Ma, S. Mabruk, G. Rabani, V. M. Rotello, V. Sindelar, P. Woisel, Tetrahedron Lett. 2006, 47, 3763-3766.

[7] K. Motesharei, D. C. Myles, J. Am. Chem. Soc. 1998, 120, 7328-7336.

[8] J. J. Garcia-Lopez, S. Zapotoczny, P. Timmerman, F. C. J. M. van Veggel, G. J. Vancso, M. Crego-Calama, D. N. Reinhoudt, Chem. Commun. 2003, 352-353.

[9] R. Zirbs, F. Kienberger, P. Hinterdorfer, W. H. Binder, Langmuir 2005, 21, 84148421.

[10] X. Yu, B. Samanta, H. Xu, P. Arumugam, Y. Ofir, B. J. Jordan, V. M. Rotello, Small 2009, 5, 86-89. 
[11] C. Subramani, S. Dickert, Y. C. Yeh, M. T. Tuominen, V. M. Rotello, Langmuir 2011, 27, 1543-1545.

[12] M. Maskus, H. D. Abruna, Langmuir 1996, 12, 4455-4462.

[13] K. Kanaizuka, M. Murata, Y. Nishimori, I. Mori, K. Nishio, H. Masuda, H. Nishihara, Chem. Lett. 2005, 34, 534-535.

[14] C. Haensch, M. Chiper, C. Ulbricht, A. Winter, S. Hoeppener, U. S. Schubert, Langmuir 2008, 24, 12981-12985.

[15] S. A. Levi, P. Guatteri, F. C. J. M. van Veggel, G. J. Vancso, E. Dalcanale, D. N. Reinhoudt, Angew. Chem. Int. Ed. 2001, 40, 1892-1896.

[16] E. Menozzi, R. Pinalli, E. A. Speets, B. J. Ravoo, E. Dalcanale, D. N. Reinhoudt, Chem. Eur. J. 2004, 10, 2199-2206.

[17] I. Doron-Mor, H. Cohen, S. R. Cohen, R. Popovitz-Biro, A. Shanzer, A. Vaskevich, I. Rubinstein, Langmuir 2004, 20, 10727-10733.

[18] H. C. Yang, K. Aoki, H. G. Hong, D. D. Sackett, M. F. Arendt, S. L. Yau, C. M. Bell, T. E. Mallouk, J. Am. Chem. Soc. 1993, 115, 11855-11862.

[19] D. L. Thomsen, T. Phely-Bobin, F. Papadimitrakopoulos, J. Am. Chem. Soc. 1998, $120,6177-6178$.

[20] E. Soto, J. C. MacDonald, C. G. F. Cooper, W. G. McGimpsey, J. Am. Chem. Soc. 2003, 125, 2838-2839.

[21] a) A. C. Templeton, F. P. Zamborini, W. P. Wuelfing, R. W. Murray, Langmuir 2000, 16, 6682-6688; b) W. P. Wuelfing, F. P. Zamborini, A. C. Templeton, X. G. Wen, H. Yoon, R. W. Murray, Chem. Mater. 2001, 13, 87-95; c) F. P. Zamborini, J. F. Hicks, R. W. Murray, J. Am. Chem. Soc. 2000, 122, 4514-4515; d) F. P. Zamborini, M. C. Leopold, J. F. Hicks, P. J. Kulesza, M. A. Malik, R. W. Murray, J. Am. Chem. Soc. 2002, 124, 8958-8964. 
[22] M. Wanunu, R. Popovitz-Biro, H. Cohen, A. Vaskevich, I. Rubinstein, J. Am. Chem. Soc. 2005, 127, 9207-9215.

[23] S. W. Chen, R. J. Pei, T. F. Zhao, D. J. Dyer, J. Phys. Chem. B 2002, 106, 1903-1908.

[24] A. Friggeri, H. J. van Manen, T. Auletta, X. M. Li, S. Zapotoczny, H. Schonherr, G. J. Vancso, J. Huskens, F. C. J. M. van Veggel, D. N. Reinhoudt, J. Am. Chem. Soc. 2001, 123, 6388-6395.

[25] N. Crivillers, M. Mas-Torrent, S. Perruchas, N. Roques, J. Vidal-Gancedo, J. Veciana, C. Rovira, L. Basabe-Desmonts, B. J. Ravoo, M. Crego-Calama, D. N. Reinhoudt, Angew. Chem. Int. Ed. 2007, 46, 2215-2219.

[26] E. J. Calvo, F. Battaglini, C. Danilowicz, A. Wolosiuk, M. Otero, Faraday Discuss. 2000, 47-65.

[27] Y. Saga, T. Y. Kim, T. Hisai, H. Tamiaki, Thin Solid Films 2006, 500, 278-282.

[28] T. P. Burgin, J. C. Lewenstein, D. Werho, Langmuir 2005, 21, 6596-6602.

[29] J. P. Opatkiewicz, M. C. LeMieux, Z. N. Bao, ACS Nano 2010, 4, 1167-1177.

[30] F. Auer, M. Scotti, A. Ulman, R. Jordan, B. Sellergren, J. Garno, G. Y. Liu, Langmuir 2000, 16, 7554-7557.

[31] A. Gole, C. J. Orendorff, C. J. Murphy, Langmuir 2004, 20, 7117-7122.

[32] A. Gole, S. R. Sainkar, M. Sastry, Chem. Mater. 2000, 12, 1234-1239.

[33] a) K. Akamatsu, A. Kimura, H. Matsubara, S. Ikeda, H. Nawafune, Langmuir 2005, 21, 8099-8102; b) C. A. Fustin, G. Glasser, H. W. Spiess, U. Jonas, Langmuir 2004, 20, 9114-9123; c) H. Tanaka, M. Mitsuishi, T. Miyashita, Langmuir 2003, 19, $3103-$ 3105.

[34] a) J. W. Zheng, Z. H. Zhu, H. F. Chen, Z. F. Liu, Langmuir 2000, 16, 4409-4412; b) D. J. Zhou, A. Bruckbauer, C. Abell, D. Klenerman, D. J. Kang, Adv. Mater. 2005, $17,1243-1248$. 
[35] P. Maury, M. Peter, V. Mahalingam, D. N. Reinhoudt, J. Huskens, Adv. Funct. Mater. 2005, 15, 451-457.

[36] a) L. M. Demers, C. A. Mirkin, Angew. Chem. Int. Ed. 2001, 40, 3069-3071; b) J. C. Garno, Y. Y. Yang, N. A. Amro, S. Cruchon-Dupeyrat, S. W. Chen, G. Y. Liu, Nano Lett. 2003, 3, 389-395; c) S. Hoeppener, R. Maoz, S. R. Cohen, L. F. Chi, H. Fuchs, J. Sagiv, Adv. Mater. 2002, 14, 1036-1041; d) S. Hoeppener, U. S. Schubert, Small 2005, 1, 628-632; e) S. T. Liu, R. Maoz, J. Sagiv, Nano Lett. 2004, 4, 845-851; f) S. T. Liu, R. Maoz, G. Schmid, J. Sagiv, Nano Lett. 2002, 2, 1055-1060; g) R. Maoz, S. R. Cohen, J. Sagiv, Adv. Mater. 1999, 11, 55-61; h) R. Maoz, E. Frydman, S. R. Cohen, J. Sagiv, Adv. Mater. 2000, 12, 725-731; i) D. Wouters, U. S. Schubert, J. Mater. Chem. 2005, 15, 2353-2355.

[37] a) A. Friggeri, F. C. J. M. van Veggel, D. N. Reinhoudt, Chem. Eur. J. 1999, 5, 35953602; b) A. Friggeri, F. C. J. M. van Veggel, D. N. Reinhoudt, R. P. H. Kooyman, Langmuir 1998, 14, 5457-5463; c) B. H. Huisman, R. P. H. Kooyman, F. C. J. M. vanVeggel, D. N. Reinhoudt, Adv. Mater. 1996, 8, 561-564.

[38] J. D. Faull, V. K. Gupta, Langmuir 2001, 17, 1470-1476.

[39] S. Y. Jon, N. Selvapalam, D. H. Oh, J. K. Kang, S. Y. Kim, Y. J. Jeon, J. W. Lee, K. Kim, J. Am. Chem. Soc. 2003, 125, 10186-10187.

[40] Q. An, G. T. Li, C. G. Tao, Y. Li, Y. G. Wu, W. X. Zhang, Chem. Commun. 2008, 1989-1991.

[41] J. F. Young, H. D. Nguyen, L. T. Yang, J. Huskens, P. Jonkheijm, L. Brunsveld, ChemBioChem 2010, 11, 180-183.

[42] a) T. Auletta, B. Dordi, A. Mulder, A. Sartori, S. Onclin, C. M. Bruinink, M. Peter, C. A. Nijhuis, H. Beijleveld, H. Schonherr, G. J. Vancso, A. Casnati, R. Ungaro, B. J. Ravoo, J. Huskens, D. N. Reinhoudt, Angew. Chem. Int. Ed. 2004, 43, 369-373; b) I. 
A. Banerjee, L. T. Yu, H. Matsui, J. Am. Chem. Soc. 2003, 125, 9542-9543; c) M. R. de Jong, J. Huskens, D. N. Reinhoudt, Chem. Eur. J. 2001, 7, 4164-4170; d) A. Fragoso, J. Caballero, E. Almirall, R. Villalonga, R. Cao, Langmuir 2002, 18, 50515054; e) C. A. Nijhuis, J. Huskens, D. N. Reinhoudt, J. Am. Chem. Soc. 2004, 126, $12266-12267$.

[43] C. A. Nijhuis, K. A. Dolatowska, B. J. Ravoo, J. Huskens, D. N. Reinhoudt, Chem. Eur. J. 2007, 13, 69-80.

[44] X. Y. Ling, D. N. Reinhoudt, J. Huskens, Chem. Mater. 2008, 20, 3574-3578.

[45] M. J. W. Ludden, X. Li, J. Greve, A. van Amerongen, M. Escalante, V. Subramaniam, D. N. Reinhoudt, J. Huskens, J. Am. Chem. Soc. 2008, 130, 6964-6973.

[46] A. Mulder, S. Onclin, M. Peter, J. P. Hoogenboom, H. Beijleveld, J. ter Maat, M. F. Garcia-Parajo, B. J. Ravoo, J. Huskens, N. F. van Hulst, D. N. Reinhoudt, Small 2005, $1,242-253$.

[47] a) O. Crespo-Biel, B. Dordi, P. Maury, M. Peter, D. N. Reinhoudt, J. Huskens, Chem. Mater. 2006, 18, 2545-2551; b) P. Maury, M. Escalante, M. Peter, D. N. Reinhoudt, V. Subramaniam, J. Huskens, Small 2007, 3, 1584-1592; c) P. Maury, M. Peter, O. Crespo-Biel, X. Y. Ling, D. N. Reinhoudt, J. Huskens, Nanotechnology 2007, 18, -.

[48] A. Mulder, T. Auletta, A. Sartori, S. Del Ciotto, A. Casnati, R. Ungaro, J. Huskens, D. N. Reinhoudt, J. Am. Chem. Soc. 2004, 126, 6627-6636.

[49] O. Crespo-Biel, C. W. Lim, B. J. Ravoo, D. N. Reinhoudt, J. Huskens, J. Am. Chem. Soc. 2006, 128, 17024-17032.

[50] C. W. Lim, O. Crespo-Biel, M. C. A. Stuart, D. N. Reinhoudt, J. Huskens, B. J. Ravoo, Proceed. Natl. Acad. Sci. USA 2007, 104, 6986-6991.

[51] M. J. W. Ludden, M. Peter, D. N. Reinhoudt, J. Huskens, Small 2006, 2, 1192-1202.

[52] S. Onclin, J. Huskens, B. J. Ravoo, D. N. Reinhoudt, Small 2005, 1, 852-857. 
[53] a) M. J. W. Ludden, A. Mulder, R. Tampe, D. N. Reinhoudt, J. Huskens, Angew. Chem. Int. Ed. 2007, 46, 4104-4107; b) M. L. W. Ludden, A. Mulder, K. Schulze, V. Subramaniam, R. Tampe, J. Huskens, Chem. Eur. J. 2008, 14, 2044-2051.

[54] H. Xu, R. Hong, T. X. Lu, O. Uzun, V. M. Rotello, J. Am. Chem. Soc. 2006, 128, $3162-3163$.

[55] K. Kobayashi, N. Tonegawa, S. Fujii, J. Hikida, H. Nozoye, K. Tsutsui, Y. Wada, M. Chikira, M. Haga, Langmuir 2008, 24, 13203-13211.

[56] T. Hatano, A. Ikeda, T. Akiyama, S. Yamada, M. Sano, Y. Kanekiyo, S. Shinkai, J. Chem. Soc., Perkin Trans. 2 2000, 5, 909-912.

[57] A. Langner, S. L. Tait, N. Lin, R. Chandrasekar, M. Ruben, K. Kern, Angew. Chem. Int. Ed. 2008, 47, 8835-8838.

[58] F. Tancini, D. Genovese, M. Montalti, L. Cristofolini, L. Nas, L. Prodi, E. Dalcanale, J. Am. Chem. Soc. 2010, 132, 4781-4789.

[59] M. E. Canas-Ventura, K. Ait-Mansour, P. Ruffieux, R. Rieger, K. Mullen, H. Brune, R. Fasel, ACS Nano 2011, 5, 457-469. 
Chapter 2 


\section{Chapter 3}

\section{Expression of Sensitized $\mathrm{Eu}^{3+}$ Luminescence at a Multivalent Interface*}

The assembly of a mixture of guest-functionalized antenna and Eu ${ }^{3+}$-complexed ligand molecules in a patterned fashion onto a receptor surface was shown to provide local and efficient sensitized Eu ${ }^{3+}$ emission. Coordination of a carboxylate group of the antenna to the $\mathrm{Eu}^{3+}$ center and noncovalent anchoring of both components to the receptor surface appeared to be prerequisites for efficient energy transfer. A Job plot at the surface confirmed that coordination of the antenna to the Eu ${ }^{3+}$ center occured in a 1:1 fashion. The efficiency of this intramolecular binding process is promoted by the high effective concentration of both complementary moieties at the surface. The system constitutes therefore an example of supramolecular expression of a complex consisting of several different building blocks which signals its own correct formation.

\footnotetext{
* Part of this chapter has been published in: Shu-Han Hsu, M. Deniz Yilmaz, Christian Blum, Vinod Subramaniam, David N. Reinhoudt, Aldrik H. Velders, Jurriaan Huskens, J. Am. Chem. Soc. 2009, 131, 12567-12569.
} 


\subsection{Introduction}

Self-assembly provides a unique paradigm to obtain complex and functional molecular architectures in a spontaneous process from small building blocks. ${ }^{[1]}$ Self-assembly at surfaces is particularly rewarding since the inherent immobilization allows characterization by single molecule techniques ${ }^{[2]}$ and potential embedding in a device structure. It has only been recently recognized that surfaces, in particular those functionalized with molecular recognition units, the so-called molecular printboards, offer additional benefits regarding control over molecular orientation, footprint, stability of binding, and suppression of nonspecific interactions. ${ }^{[3,4]}$ These properties are given by the fact that molecules and complexes can be bound to such surfaces via multivalent interactions, which are governed by the principle of effective molarity. ${ }^{[4]}$ When complexity is increased, ${ }^{[5]}$ here when going from one to more interaction motifs, new emerging properties can be expected. It has been shown before that the use of building blocks with orthogonal interaction motifs that self-assemble on molecular printboards can lead to the selective formation of one type of complex (from a large number of potential complexes) consisting of more than two different building blocks, ${ }^{[6]}$ and control over supramolecular aggregation of receptor-functionalized vesicles. ${ }^{[7]}$ Here we show, for the first time, the spontaneous formation of such a complex that signals its own correct assembly, by expressing sensitized lanthanide luminescence. The focus is on addressing the exact stoichiometry of the complex and its signaling properties.

The trivalent cations of several lanthanides and their complexes with organic ligands are known to exhibit characteristic emission line shapes, relatively long luminescence lifetimes, and a strong sensitivity towards quenching by high frequency, e.g. O-H, oscillators. ${ }^{[8]}$ Because of their sharp, narrow absorption peaks and low absorption coefficients, lanthanide ions are usually excited via energy transfer from an excited organic chromophore (the antenna or sensitizer), that has a much higher absorption coefficient. ${ }^{[9]}$ The energy transfer process is strongly distance dependent and limits the practical lanthanide- 
antenna distance to $<5 \AA \AA^{[10]}$ Photophysical properties of lanthanide complexes in solution have been extensively studied. In a supramolecular example, an EDTA-based ligand with $\beta$ cyclodextrin $(\beta-\mathrm{CD})$ binding sites showed sensitized $\mathrm{Eu}^{3+}$ emission by noncovalent capture of an organic sensitizer. ${ }^{[12]}$ The immobilization and photophysical properties of lanthanide complexes on surfaces has not been investigated, except for some recent examples in which a $\mathrm{Eu}^{3+}$ complex was bound to a particle surface, ${ }^{[13]}$ especially for sensor applications. ${ }^{[14]}$

Here, we employ antenna-sensitized $\mathrm{Eu}^{3+}$ luminescence based on host-guest interactions on the molecular printboard, which allows qualitative and quantitative studies of the complexation of four different building blocks (Figure 3.1a): an EDTA-based ligand for binding $\mathrm{a}^{3+}$ ion and the receptor surface, a naphthalene-based antenna molecule with receptor-binding moieties and with a carboxylate group for coordination to the $\mathrm{Eu}^{3+}$ ion, the $\mathrm{Eu}^{3+}$ ion, and a $\beta$-CD monolayer which functions as the receptor surface. The EDTA ligand and the antenna molecule are equipped with adamantyl groups (Ad) for noncovalent anchoring to the $\beta$-CD monolayer. The $\beta$-CD monolayer is used to immobilize both the sensitizer and the $\mathrm{Eu}^{3+}$ complex, thus enforcing close proximity of the molecules and facilitating sensitized lanthanide luminescence owing to efficient energy transfer (Figure $3.1 b)$.
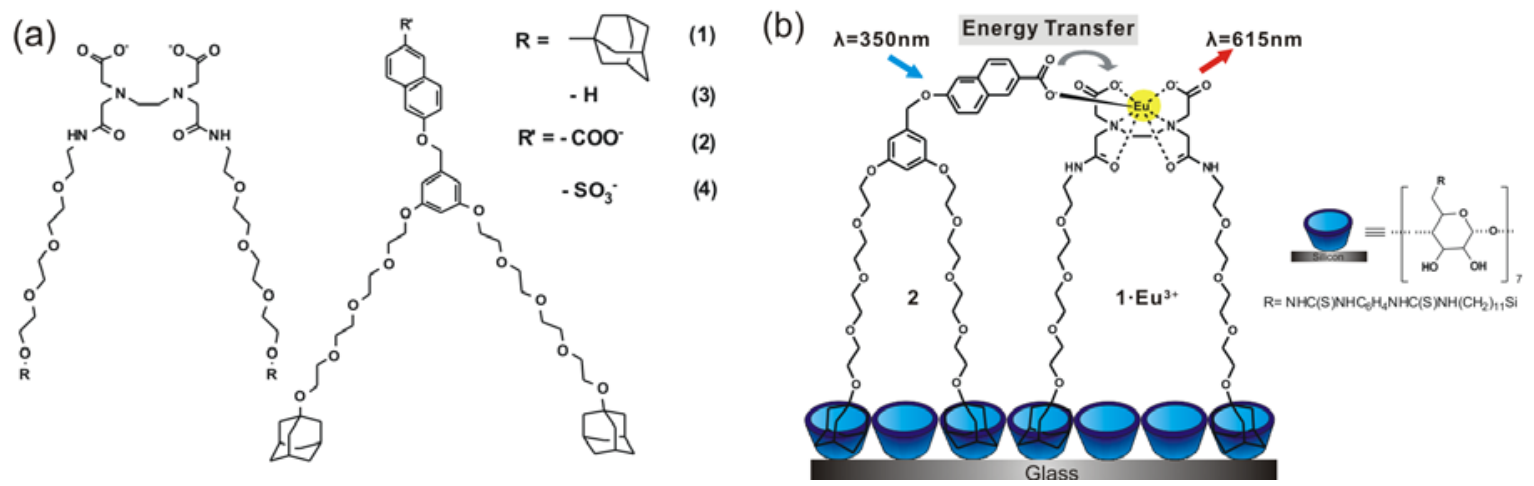

Figure 3.1 (a) EDTA-based ligands with (1) and without (3) adamantyl (Ad) moieties, and carboxylate- (2) or sulfonate- (4) modified naphthalene derivatives with Ad groups. (b) Molecular structure of the target complex on a $\beta$-CD SAM schematically showing sensitized $\mathrm{Eu}^{3+}$ luminescence. 


\subsection{Results and discussion}

\subsubsection{Synthesis}

An EDTA-based ligand 1 for binding a $\mathrm{Eu}^{3+}$ ion and the receptor surface was synthesized as outlined in Scheme 3.1, and as follows. Amino ethyl triethylene glycol adamantyl ether was reacted with ethylenediaminetetraacetic dianhydride at room temperature in dry DMF using triethylamine as a base to give the bis(adamantyl tetraethylene glycol)- functionalized ethylenediaminetetraacetic acid 1. The Eu(III) complex of compound 1 was prepared by adding a solution of $\mathrm{EuCl}_{3} \cdot 6 \mathrm{H}_{2} \mathrm{O}$ in water to a solution of $\mathbf{1}$, adjusting the $\mathrm{pH}$ to 7 with overnight stirring at rt. A slight excess of 1 relative to $\mathrm{Eu}(\mathrm{III})$ (1.03:1) was used, ensuring quantitative complexation of the lanthanide ion. Compound $\mathbf{3}$ was designed and synthesized for a control experiment by adding ligand 1 into TFA/ $\mathrm{CH}_{2} \mathrm{Cl}_{2}$ mixture, and the Eu(III) complex of compound 3 was prepared in a similar manner as 1.Eu(III). A naphthalene-based antenna molecule 2 with receptor-binding moieties and with a carboxylate group for coordination to the $\mathrm{Eu}^{3+}$ ion was synthesized in three steps. In the first step, bromoethyl triethylene glycol adamantyl ether was reacted with 3,5-dihydroxybenzyl alcohol under reflux in acetone using $\mathrm{K}_{2} \mathrm{CO}_{3}$ as a base to give compound 5 . The conversion of the hydroxyl group to the reactive bromide using $\mathrm{PBr}_{3}$ in dry toluene gave compound 6 . In the last step, reaction between compound 6 and methyl-6-hydroxy-2-naphthoate using $\mathrm{K}_{2} \mathrm{CO}_{3}$ as a base gave the methyl ester of compound 2 . The cleavage of methyl ester to free carboxylic acid by $\mathrm{NaOMe}$ produced compound 2. Compound 4 was synthesized as a reference compound by the reaction between compound $\mathbf{6}$ and 6-hydroxy-2-naphthalene sulfonic acid sodium salt using $\mathrm{NaOMe}$ as a base. 

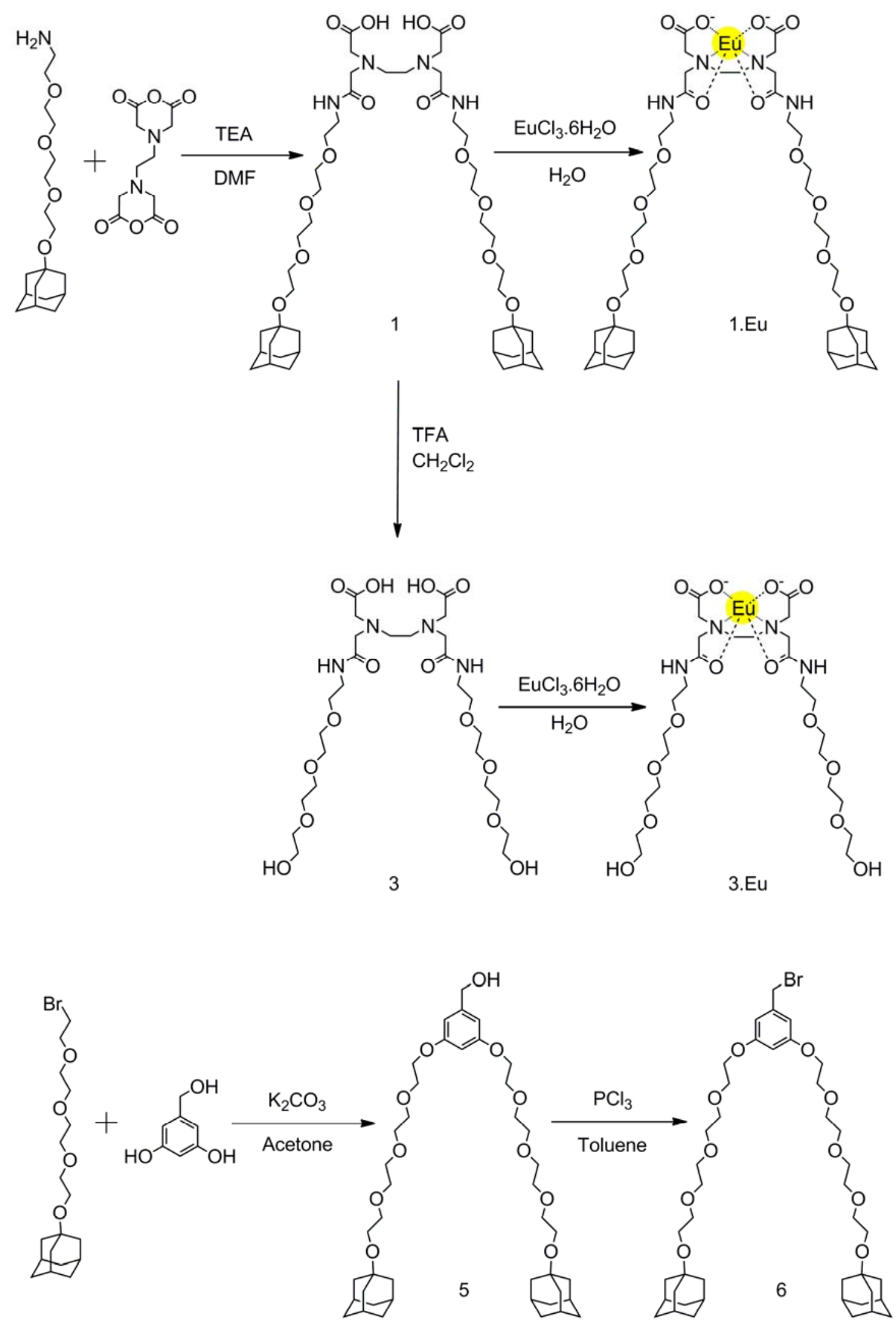

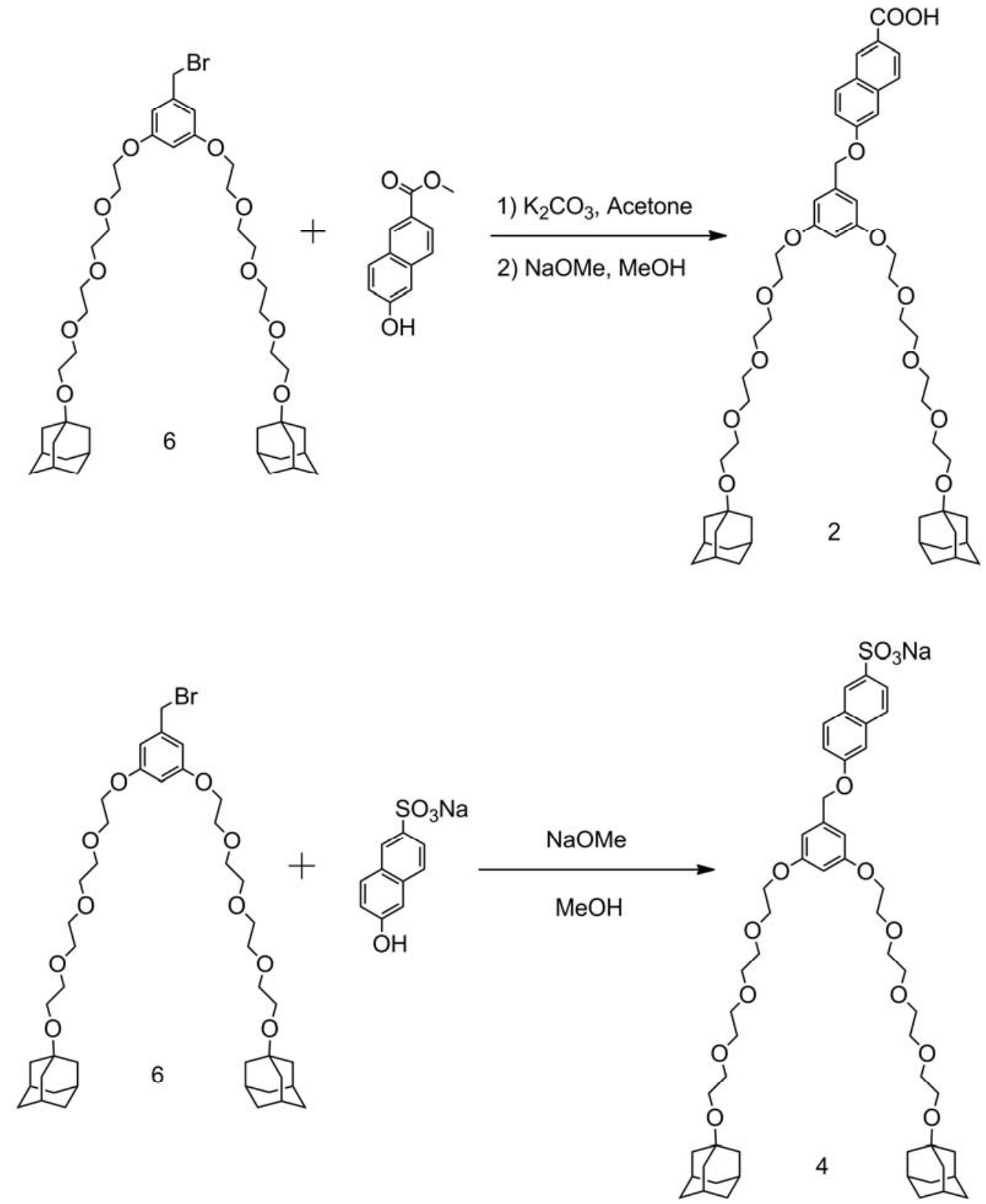

Scheme 3.1 Synthesis routes towards the building blocks used in this study 


\subsubsection{Complex formation in solution}

Fluorescence spectroscopy measurements were performed to study the sensitized luminescence of the lanthanide complex $1 \cdot \mathrm{Eu}^{3+}$ in solution in the absence and presence of antenna 2 (Figure 3.2). The fluorescence spectrum of a $10 \mu \mathrm{M}$ solution of $\mathbf{1} \cdot \mathrm{Eu}^{3+}$ in $\mathrm{H}_{2} \mathrm{O}$ did not show the characteristic $\mathrm{Eu}^{3+}$ emission at $615 \mathrm{~nm}$, not even upon addition of an equimolar amount of 2, excited at $350 \mathrm{~nm}$ while recording the luminescence spectrum between $350 \mathrm{~nm}$ and $650 \mathrm{~nm}$. The broad band from $350 \mathrm{~nm}$ to $500 \mathrm{~nm}$ was attributed to the emission of 2 .

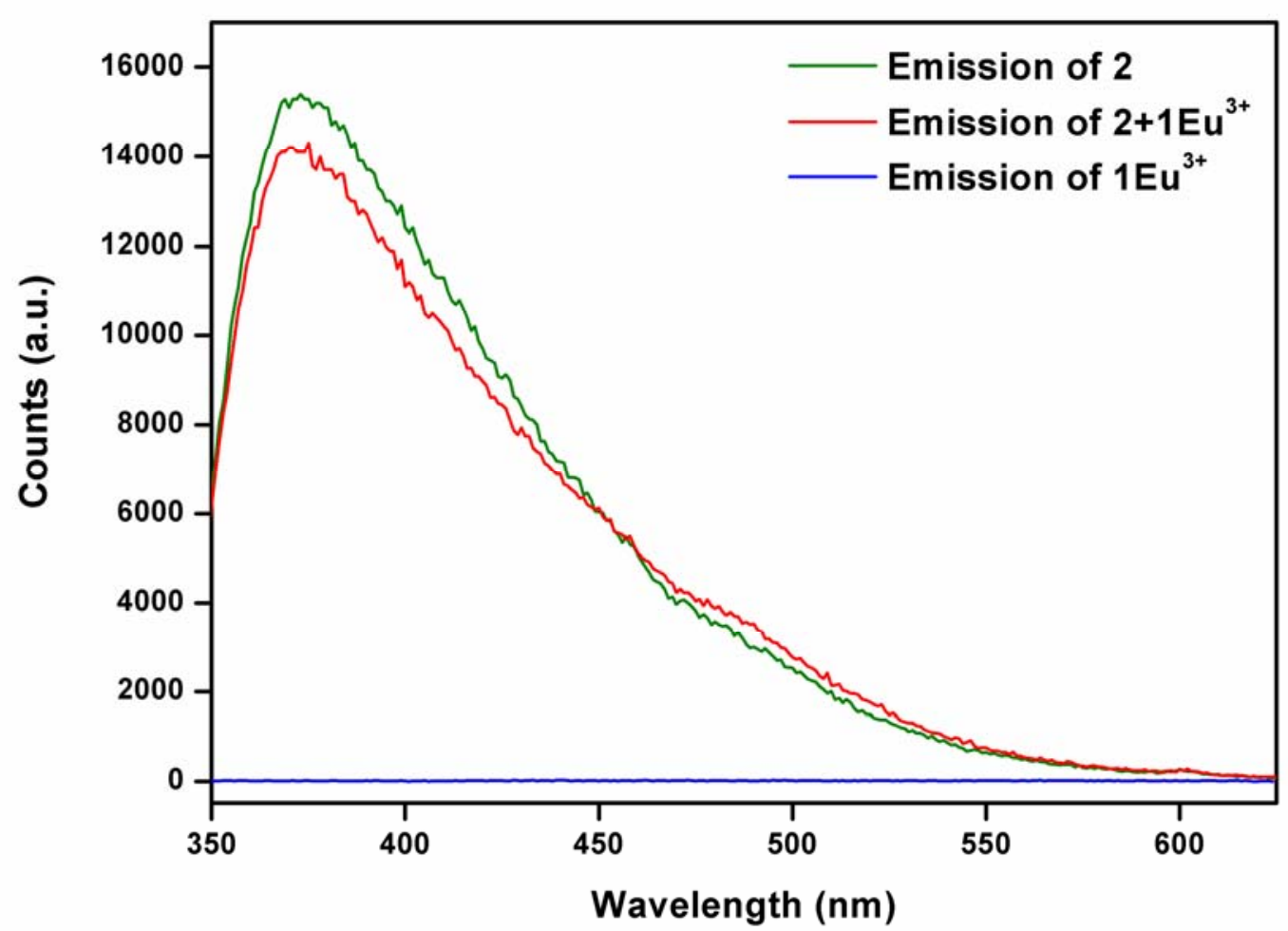

Figure 3.2. Luminescence emission spectra of $10 \mu \mathrm{M}$ solutions of $\mathbf{2}, \mathbf{2}+\mathbf{1} \cdot \mathrm{Eu}^{3+}$ and $\mathbf{1} \cdot \mathrm{Eu}^{3+}$ in $\mathrm{H}_{2} \mathrm{O}$ excited at $350 \mathrm{~nm}$. 


\subsubsection{Complex formation at the molecular printboard}

Microcontact printing $(\mu \mathrm{CP})$ onto $\beta$-CD monolayers, resulting in host-guest complex formation, was used to generate surface patterns of the complex on the receptor surface. Two methods were applied to immobilize the complex onto the surface (Scheme 3.2): (i) the surfaces were patterned by printing an equimolar ratio of $\mathbf{1} \cdot \mathrm{Eu}^{3+}$ and 2 onto the $\beta$-CD SAM $\left(\mathbf{i}_{\mathbf{a}}\right)$, followed by backfilling the nonprinted area with $\mathbf{1} \cdot \mathrm{Eu}^{3+}$, which was used as an internal reference (i $\mathbf{i}_{\mathbf{b}}$ ); (ii) the surfaces were patterned by printing different ratios of $\mathbf{1}$ and $\mathbf{2}$ (ii $\mathbf{i}_{\mathbf{a}}$ ), followed by solution immersion in aqueous $\mathrm{EuCl}_{3}\left(\mathbf{i i}_{\mathbf{b}}\right)$. The solution immersion steps $\left(\mathbf{i}_{\mathbf{b}}\right.$ and $\left.\mathbf{i i}_{\mathbf{b}}\right)$ were performed in the absence of $\beta$-CD in solution in order to prevent exchange of 2 by $\mathbf{1} \cdot \mathrm{Eu}^{3+}\left(\mathbf{i}_{\mathbf{b}}\right)$ and desorption of $\mathbf{1}$ and $\mathbf{2}\left(\mathbf{i i}_{\mathbf{b}}\right){ }^{[10]}$

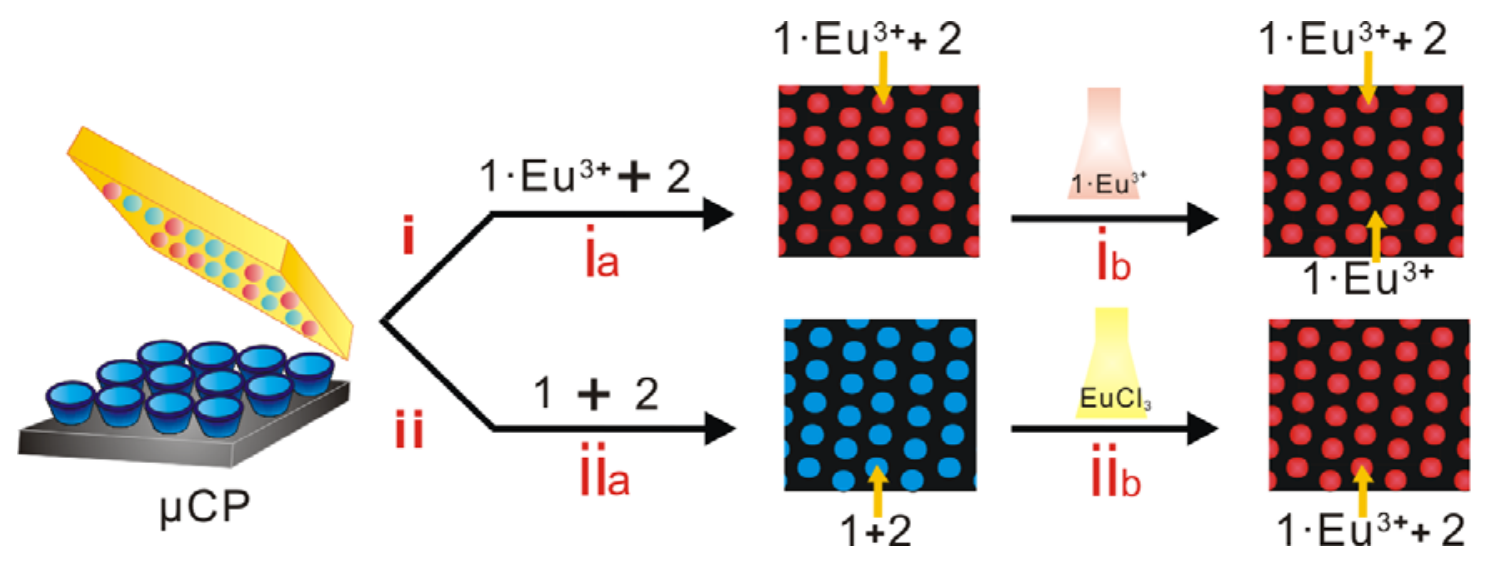

Scheme 3.2 Schematic representation of two immobilization procedures (i and ii) of the Ad ligands $\mathbf{1}$ and $\mathbf{2}$ without $\left(\mathbf{i}_{\mathrm{a}}, \mathbf{i i}_{\mathrm{a}}\right)$ or with $\left(\mathbf{i}_{\mathrm{b}}, \mathbf{i}_{\mathrm{b}}\right)$ a solution step for backfilling with $\mathbf{1} \cdot \mathrm{Eu}^{3+}$ in the nonprinted area $\left(\mathbf{i}_{\mathbf{b}}\right)$ or complexation of $\mathbf{1}$ with $\mathrm{Eu}^{3+}\left(\mathbf{i i}_{\mathbf{b}}\right)$.

As an initial indication for energy transfer at the molecular printboard patterned using method $\mathbf{i}$, red emission measured using filter $\mathbf{R}^{[11]}$ only appeared in the areas where both $\mathbf{1} \cdot \mathrm{Eu}^{3+}$ and $\mathbf{2}$ are present (Figure 3.3a), which demonstrates qualitatively the occurrence of sensitized $\mathrm{Eu}^{3+}$ luminescence. Local emission spectra were recorded to further characterize 
the patterned surface of $\mathbf{1} \cdot \mathrm{Eu}^{3+}$ and $\mathbf{2}$ (Figure $3.3 \mathrm{~b}$ ). The emission spectra were selectively collected from both the patterned and nonpatterned areas upon excitation in the UV (step $\mathbf{i}_{\mathbf{b}}$ ). From the nonpatterned areas, the observed $\mathrm{Eu}^{3+}$ emission is faint and can be attributed to inefficient direct UV excitation of $\mathbf{1} \cdot \mathrm{Eu}^{3+}$ alone. However, a significantly higher intensity of $\mathrm{Eu}^{3+}$ emission is observed in the $\mathbf{1} \cdot \mathrm{Eu}^{3+} / 2$ patterned area. Clearly the emission of $\mathrm{Eu}^{3+}$ is amplified in the area where energy transfer occurred between the naphthalene antenna and the lanthanide complex. Considering also the fact that twice as much $\mathbf{1} \cdot \mathrm{Eu}^{3+}$ is expected to be present in the nonpatterned area with respect to the printed areas, comparing the intensities at $614 \mathrm{~nm}$, an amplification of a factor of 54 is found between the patterned and nonpatterned areas.

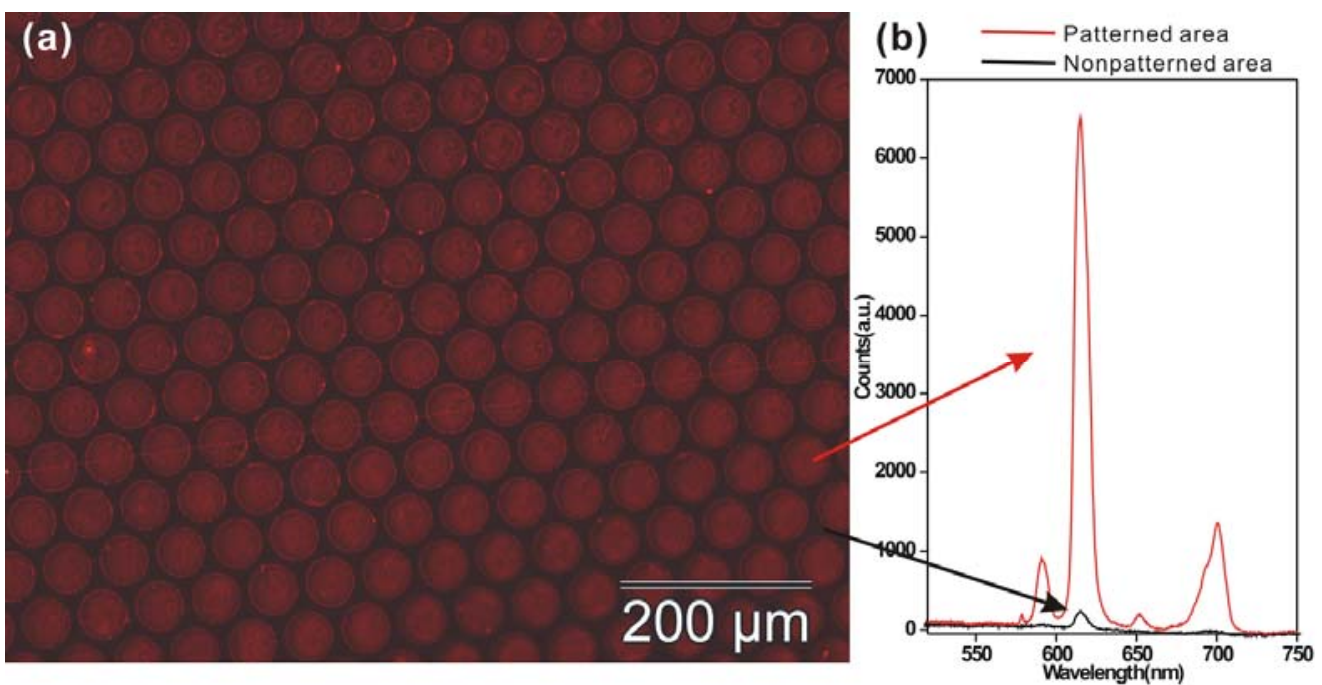

Figure 3.3 (a) Fluorescence microscopy image (left, using filter $\mathbf{R}$ ) of $50 \mu \mathrm{m}$ dots on a $\beta$-CD monolayer obtained by $\mu \mathrm{CP}$ of an equimolar ratio of $\mathbf{1} \cdot \mathrm{Eu}^{3+}$ and $\mathbf{2}$ for $30 \mathrm{~min}$ (step $\mathbf{i}_{\mathbf{a}}$ ) and subsequent incubation in a solution with $\mathbf{1} \cdot \mathrm{Eu}^{3+}$ for $30 \mathrm{~min}$ (step $\mathbf{i}_{\mathbf{b}}$ ), (b) and local emission spectra from the patterned and nonpatterned areas (right), both illustrating the enhanced $\mathrm{Eu}^{3+}$ emission in the patterned areas. 
In contrast, when using reference compound $\mathbf{4}$, a naphthalene moiety bearing a sulfonate group instead of the carboxylate in 2, no sensitized $\mathrm{Eu}^{3+}$ luminescence was observed (Figure 3.4A and B). Since the sulfonate group is not basic enough to bind a lanthanide ion, this shows that direct coordination of the carboxylate of $\mathbf{2}$ to the $\mathrm{Eu}^{3+}$ center is involved to obtain efficient energy transfer. A similar observation was made in solution. ${ }^{[12]}$ Moreover, when an EDTA-based complex without the adamantyl functionalities was used, 3. $\mathrm{Eu}^{3+}$, no sensitization of the $\mathrm{Eu}^{3+}$ luminescence was observed (Figure $3.4 \mathrm{C}$ and D). This control experiment shows that direct coordination of the carboxylate is too weak to occur on its own, and has to be assisted by anchoring of both ligands on the receptor surface in order to have the high effective concentration ${ }^{[2,3]}$ promote the direct coordination, leading to efficient energy transfer.
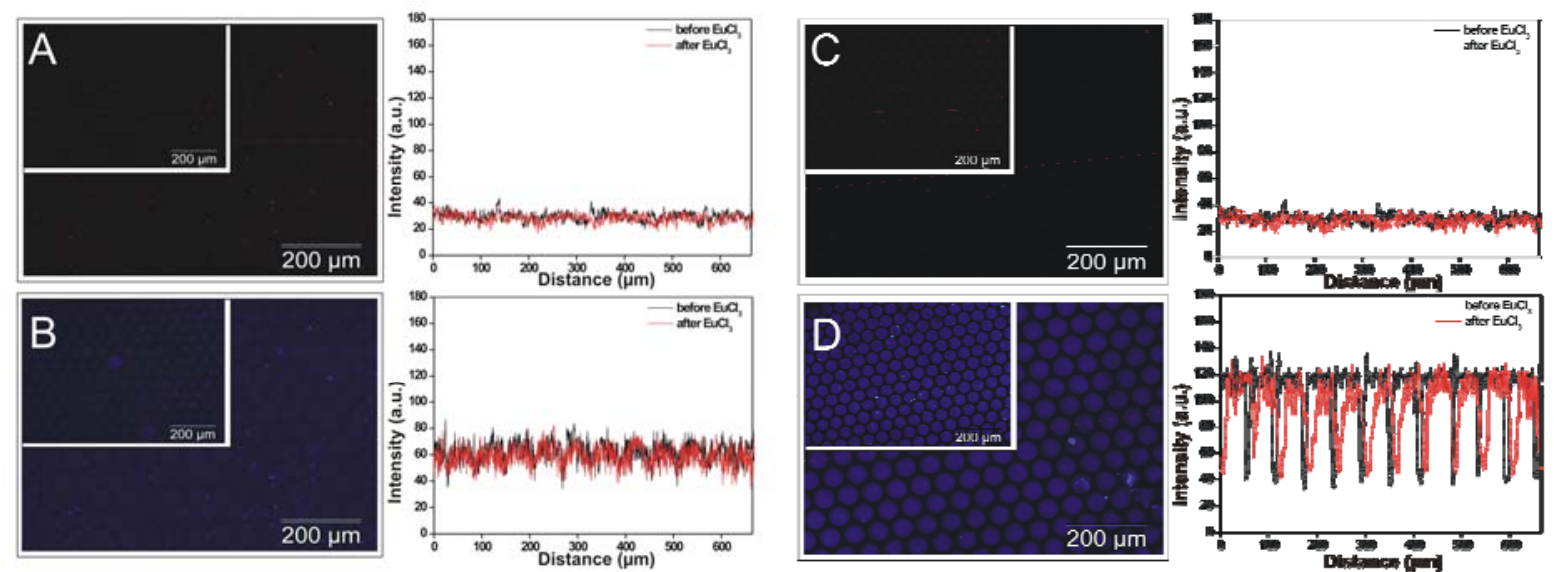

Figure 3.4 Fluorescence microscopy images $(884 \mu \mathrm{m}$ x $666 \mu \mathrm{m})$ of $50 \mu \mathrm{m}$ dots at a $\beta$-CD SAM made by $\mu \mathrm{CP}$ of a mixture of $\mathbf{1}$ and $\mathbf{4}$ for $30 \mathrm{~min}$ before (insets) and after (main images) immersion in a solution of $\mathrm{EuCl}_{3}$ for $30 \mathrm{~min}$, monitoring $\mathrm{Eu}^{3+}$ (A) and antenna (B) emission; a solution of 2 for $30 \mathrm{~min}$, before (insets) and after (main images) immersion in a solution of $3 \cdot \mathrm{Eu}^{3+}$ for $30 \mathrm{~min}$, monitoring $\mathrm{Eu}^{3+}(\mathrm{C})$ and antenna (D) emission. 
To quantify the energy transfer efficiency between naphthalene and the lanthanide complexes, the naphthalene emission lifetimes were determined in the absence and presence of $\mathrm{Eu}^{3+}$. To obtain sufficient signal, a stack of 6 glass slides coated on both sides with a monolayer of $\mathbf{2}$ or an equimolar mixture of $\mathbf{2}$ and $\mathbf{1} \cdot \mathrm{Eu}^{3+}$ was sampled at a time. The excitation source was a LED emitting at $282 \mathrm{~nm}$ at $1 \mathrm{MHz}$ repetition rate. Emitted photons were detected in a narrow wavelength range around the naphthalene emission maximum at $370 \mathrm{~nm}$ (slit $10 \mathrm{~nm}$ ). The emission lifetime from the naphthalene compound on the surface in the absence of the $\mathrm{Eu}^{3+}$ could be fitted with a double exponential with one strongly dominating component of $\tau_{1}=2.3 \mathrm{~ns}(89 \%$ relative amplitude) and a minor component of 7.0 ns $(11 \%$ relative amplitude) (Figure 3.5$)$. In the presence of $1 \cdot \mathrm{Eu}^{3+}$, the lifetime of both components significantly dropped to $1.5 \mathrm{~ns}$ and $5.3 \mathrm{~ns}$, respectively, while the relative amplitudes were preserved (91\% and 9\%). From these lifetimes we determined the dominant energy transfer efficiency to be $35 \%$ for the major and $25 \%$ for the minor component. To exclude any effect from possible energy transfer acceptor saturation due to the very long $\mathrm{Eu}^{3+}$ emission lifetime on the recorded decay characteristics, the experiment was repeated using a reduced excitation frequency of $100 \mathrm{kHz}$. The obtained results were identical to the ones obtained with $1 \mathrm{MHz}$ excitation. 


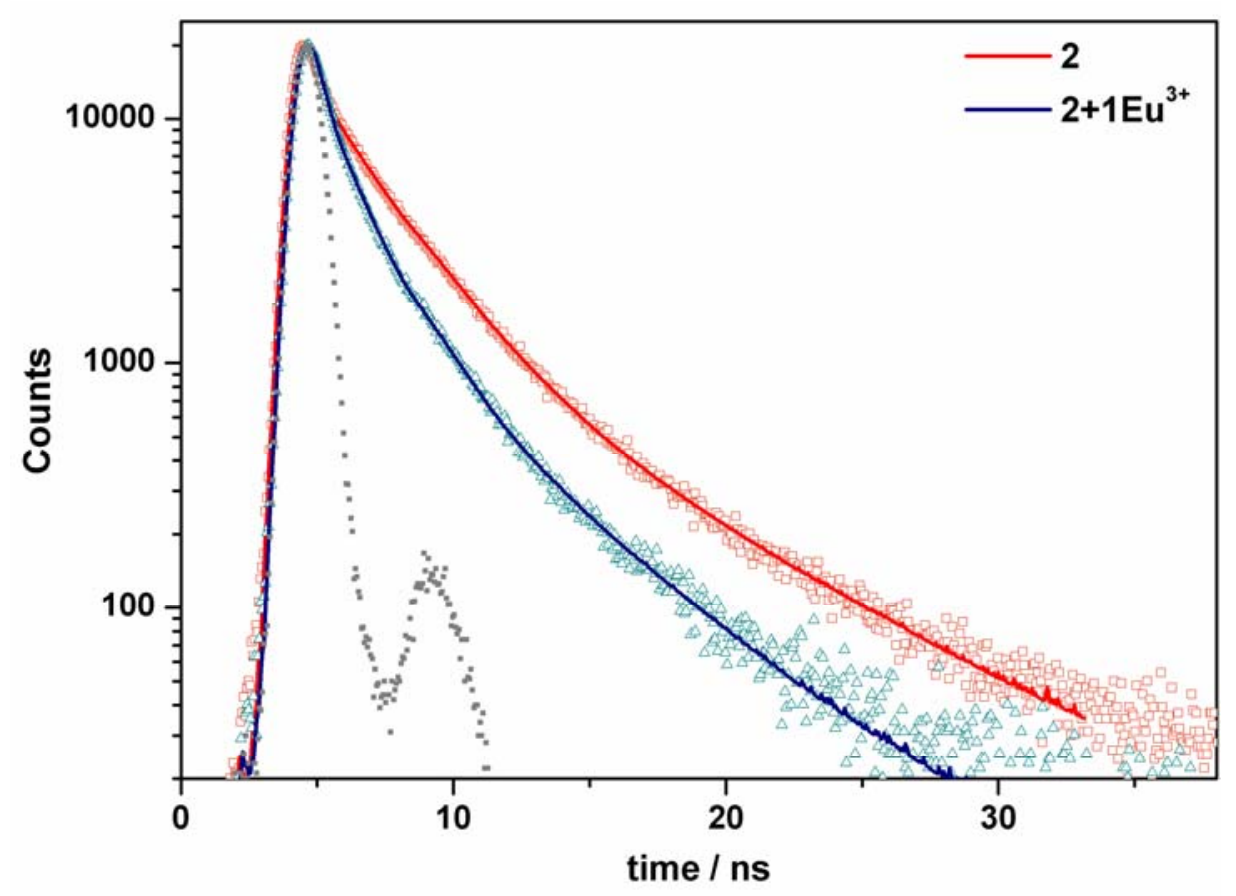

Figure 3.5 Time-resolved fluorescence measurements of 2 alone and of $\mathbf{2}$ and $\mathbf{1} \cdot \mathrm{Eu}^{3+}$ on a $\beta$ CD SAM excited at $282 \mathrm{~nm}$ with a $1 \mathrm{MHz}$ LED. The $2.3 \mathrm{~ns}$ and $7.0 \mathrm{~ns}$ lifetime components were derived from fitting the decay curves of 2 .

In order to study the stoichiometry of complexation between $\mathbf{2}$ and $\mathbf{1} \cdot \mathrm{Eu}^{3+}$, a stepwise procedure (Scheme 3.2, method ii) was applied: $\mu \mathrm{CP}$ of solution mixtures of different molar ratios of 1 and 2 was used to generate patterns on the $\beta$-CD monolayer. Directly after printing (step $\mathbf{i i}_{\mathbf{a}}$ ), the surface was imaged with fluorescence microscopy, followed by immersion in a $\mathrm{EuCl}_{3}$ solution for $30 \mathrm{~min}$ (step $\mathbf{i i}_{\mathbf{b}}$ ) and re-imaging (Figure 3.6). The fluorescence intensities of the surface antenna and $\mathrm{Eu}^{3+}$ emission were plotted as a function of the molar fraction of antenna 2 (Figure 3.7). Since the printboard ensures that the total immobilized ligand concentration $(\mathbf{1}+\mathbf{2})$ remains constant, this plot fulfills the requirements for a Job plot. 


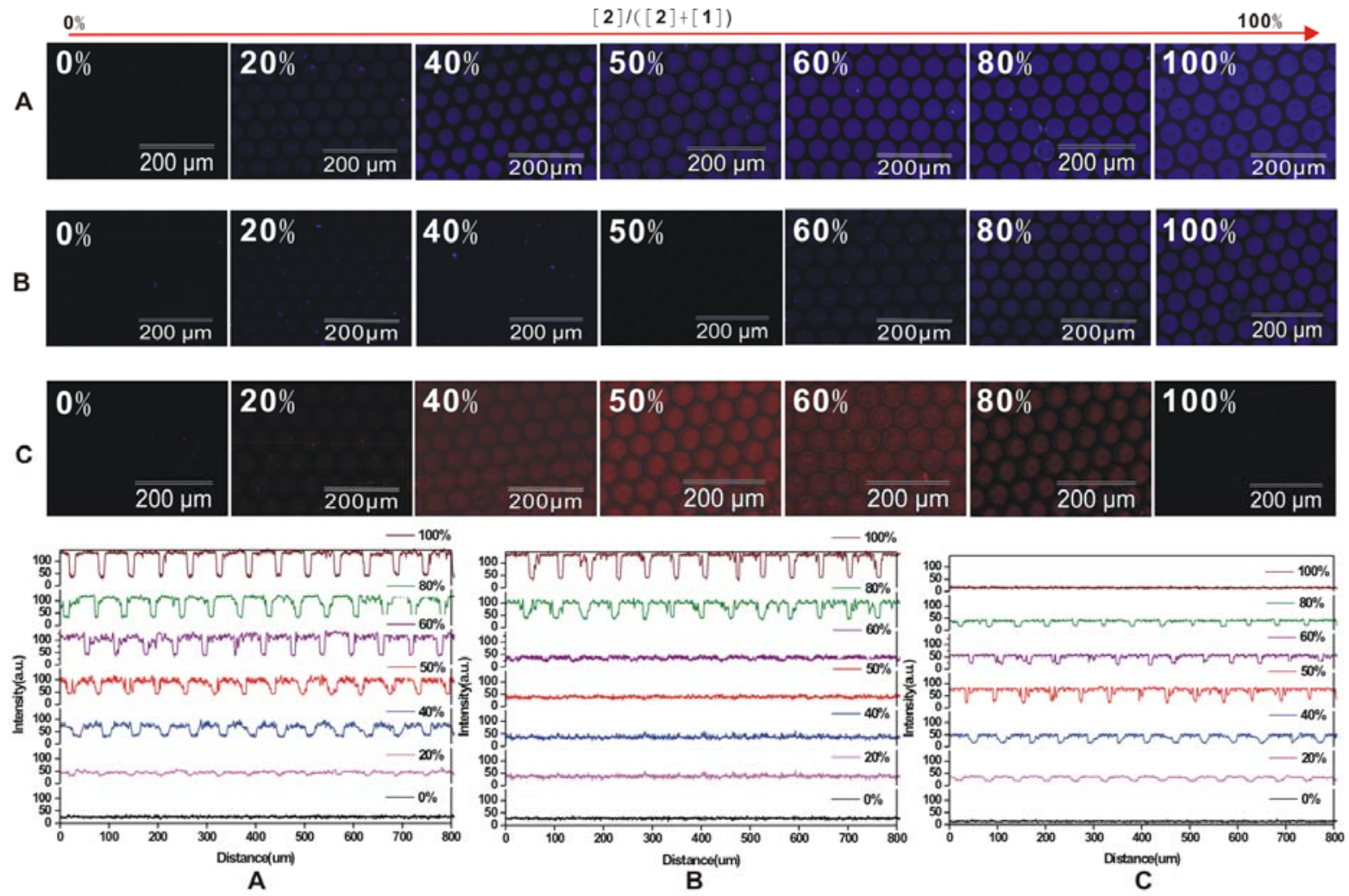

Figure 3.6 Fluorescence microscopy images of $50 \mu \mathrm{m}$ dots prepared on $\beta$-CD monolayers by $\mu \mathrm{CP}(30 \mathrm{~min}$ ) of solution mixtures of different ratios of 1 and 2 (the concentration of 2 varying from $0 \%, 20 \%, 40 \%, 50 \%, 60 \%, 80 \%$ and $100 \%$ ) (ii $\mathbf{a}_{\mathbf{a}}$ ), followed by rinsing with MilliQ water (A), and subsequently immersed in a solution of $\mathrm{EuCl}_{3}$ for $30 \mathrm{~min}\left(\mathbf{i i}_{\mathbf{b}}\right)(\mathrm{B}, \mathrm{C})$, monitoring antenna (A, B; $\mathbf{B}$ filter) and $\mathrm{Eu}^{3+}$ emission $(\mathrm{C} ; \mathbf{R}$ filter). The percentages of antenna $\mathbf{2}$ in the mixture of $\mathbf{1}$ and $\mathbf{2}$ are given in the images. The intensity profiles (bottom) are also shown before $(\mathrm{A})$ and $\operatorname{after}(\mathrm{B}, \mathrm{C}) \mathrm{EuCl}_{3}$ immersion. 


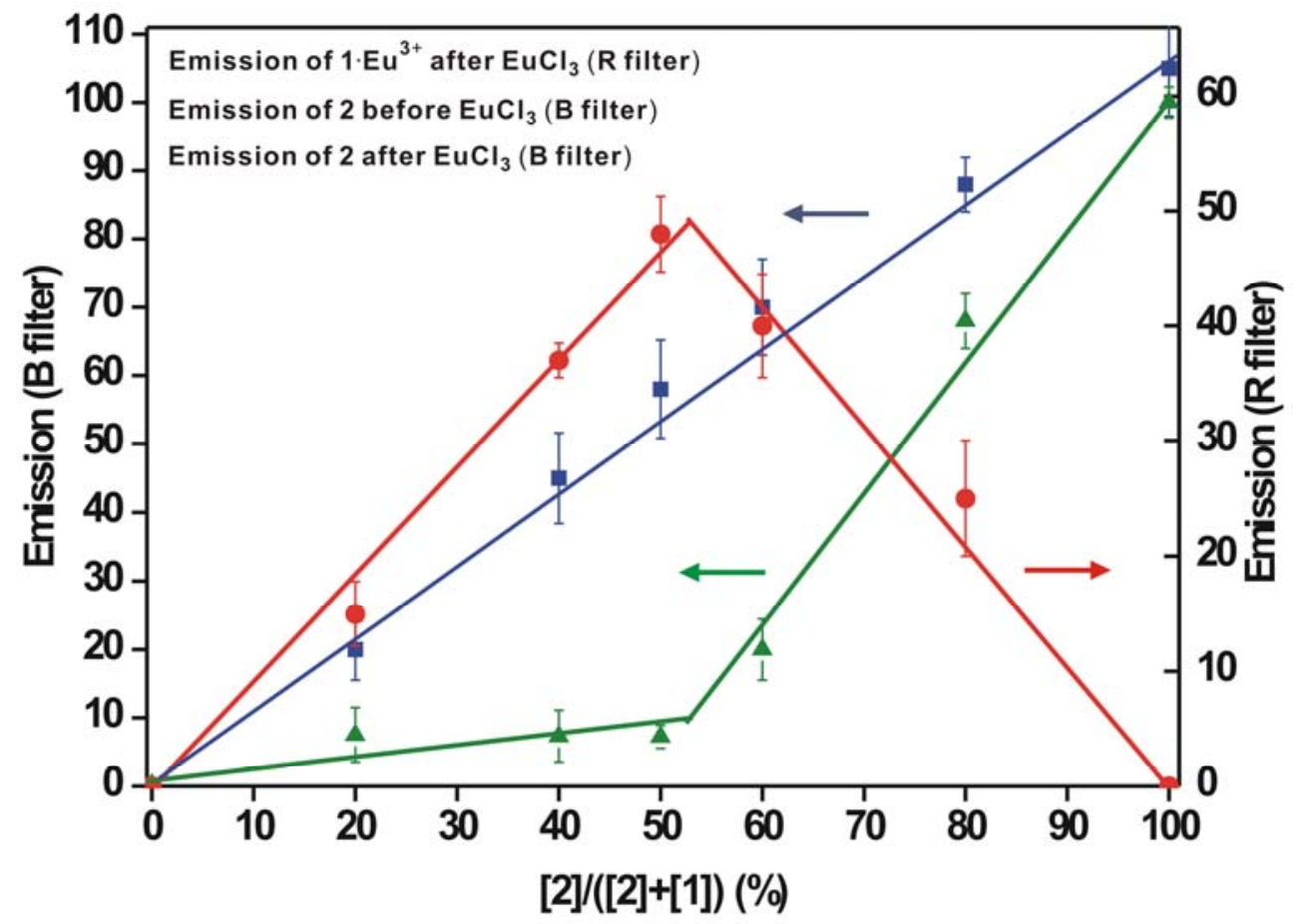

Figure 3.7 Fluorescence intensity of $\mathbf{2}$ before (blue, squares) and after (green, triangles) immersion in $\mathrm{a} \mathrm{EuCl}_{3}$ solution, and of $\mathrm{Eu}^{3+}$ emission (red, circles) after the solution step, for patterns printed from solutions with varying ratios of 2 and 1. Lines are presented for fits of the data points from $0-100 \%$ (blue line) and from $0-50 \%$ and $50-100 \%$ separately (green and red lines). The error bars represent a single standard deviation.

\subsection{Conclusions}

This work clearly demonstrates that $\mathbf{1} \cdot \mathrm{Eu}^{3+}$ and the antenna 2 form a $1: 1$ coordination pair on the $\beta$-CD SAM. The formation of the target complex is directly indicated by the occurrence of sensitized luminescence. This surface assisted luminescence amplification has potential for developing optical devices or as a sensing platform for biologically relevant anions. ${ }^{[15]}$ The system as a whole represents an example of functional expression, emerging from the combined system of all necessary components. ${ }^{[16]}$ The high specificity of the complex formation is in part attributed to the multivalency of the receptor surface which is here translated in a higher-level multivalent interface of Eu complexes with 
vacant coordination sites and antenna molecules with the complementary carboxylate groups. Another crucial factor in steering the system into the direction of the target complex is to encode the necessary information into all individual building blocks. As can be seen here, this information can be limited while still complex molecular architectures can be achieved.

\subsection{Acknowledgement}

The major part of the work presented in this chapter was performed in collaboration with Shu-Han Hsu. Christian Blum is acknowledged for performing the local fluorescence emission spectra and lifetime measurements.

\subsection{Experimental Section}

General Procedures. All reactions involving air- or moisture-sensitive reagents or intermediates were performed under an inert atmosphere of argon in glassware. Unless otherwise noted, solvents and reagents were commercially available, reagent grade and used without further purification. $\beta$-CD heptamine, amino ethyl triethylene glycol adamantyl ether and bromo triethylene glycol ethyl adamantyl ether were synthesized as described before. ${ }^{[4,17]}$ Thin layer chromatography was performed on aluminum sheets precoated with silica gel 60 F254 (Merck). Column chromatography was performed using silica gel $\left(\mathrm{SiO}_{2}\right.$, Merck, 0.040$0.063 \mathrm{~mm}, 230-240 \mathrm{mesh})$. NMR spectra were recorded on Varian AC300 spectrometer in the indicated solvent. Chemical shifts are reported in parts per million (ppm) downfield from $\left(\mathrm{CH}_{3}\right)_{4} \mathrm{Si}(\mathrm{TMS})$ as internal standard. Coupling constants are reported in hertz (Hz). Spectral splitting patterns are designated as follows: s, singlet; $d$, doublet; $t$, triplet; q, quartet; m, multiplet. ESP-MS spectra were recorded with a WATERS-LCT spectrometer. 
EDTA-based tetraethylene glycol adamantyl ether dimer 1. A solution of amino ethyl triethylene glycol adamantyl ether $(511.2 \mathrm{mg}, 1.56 \mathrm{mmol})$ and $\mathrm{Et}_{3} \mathrm{~N}(3 \mathrm{ml})$ was added dropwise to a solution of ethylenediaminetetraacetic dianhydride $(200 \mathrm{mg}, 0.78 \mathrm{mmol})$ in DMF at $0{ }^{\circ} \mathrm{C}$. The reaction mixture was stirred for 2 days at $\mathrm{rt}$. After evaporation of the solvent, the residue was dissolved in water and the $\mathrm{pH}$ was adjusted to 7 . Water was removed under reduced pressure and methanol was added to separate insoluble salts. The solvent was removed by rotary evaporator after filtration, and the residue was washed several times with diethyl ether. The product was obtained as a colorless oil, yield $80 \%$.

${ }^{1} \mathrm{H}$ NMR (300 MHz, CD $\left.3 \mathrm{OD}\right) \delta$ 3.70-3.55 (m, 28H), 3.35-3.29 (m, 8H), 3.19-3.13 (m, 4H), 2.71-2.67 (m, 4H), 2.20-2.15 (m, 6H),1.80-1.79 (m, 12H), 1.75-1.63 (m, 12H). ${ }^{13} \mathrm{C}$ NMR (75 $\left.\mathrm{MHz}, \mathrm{CD}_{3} \mathrm{OD}\right) \delta 174.9,170.3,71.0,69.5,68.7,57.6,56.6,51.2,39.8,34.8,29.3 . \mathrm{MS}$ (ESP) calcd for $\mathrm{C}_{46} \mathrm{H}_{78} \mathrm{~N}_{4} \mathrm{O}_{14}(\mathrm{M}+\mathrm{H})$ 912.1; found 912.0.

$\mathbf{E u}^{3+}$ complex of 1 . The Eu(III) complex of compound $\mathbf{1}$ was prepared by adding a solution of $\mathrm{EuCl}_{3} \cdot 6 \mathrm{H}_{2} \mathrm{O}$ in water to a solution of $\mathbf{1}$, adjusting the $\mathrm{pH}$ to 7 with overnight stirring at $\mathrm{rt}$. A slight excess of 1 relative to $\mathrm{Eu}(\mathrm{III})$ (1.03:1) was used, ensuring quantitative complexation of the lanthanide ion.

MS (ESP) calcd for $\mathrm{C}_{46} \mathrm{H}_{76} \mathrm{EuN}_{4} \mathrm{O}_{14}$ (M) 1061.0; found 1061.2.

1-Naphthoic acid-6-(3,5-di(tetraethylene glycol adamantyl ether) benzyl ether 2. A suspension of 6 (340 mg, $0.413 \mathrm{mmol}$ ), methyl-6-hydroxy-2-naphthoate (83.44 mg, 0.413 $\mathrm{mmol})$, dried potassium carbonate $(57.04 \mathrm{mg}, 0.5 \mathrm{mmol})$, and 18-crown-6 (10.5 mg, 0.04 mmol) in acetone $(50 \mathrm{~mL})$ was refluxed overnight. The solvent was evaporated and the residue was partitioned between water $(50 \mathrm{~mL})$ and diethyl ether $(50 \mathrm{~mL})$. The aqueous layer was extracted with diethyl ether $(3 \times 25 \mathrm{~mL})$ and the combined extracts were dried over 
$\mathrm{MgSO}_{4}$. The solvent was evaporated and the residue was purified by column chromatography (EtOAc, eluent) to give the methyl ester of 2 as a colorless oil $(273.2 \mathrm{mg}, 70 \%)$.

${ }^{1} \mathrm{H}$ NMR $\left(300 \mathrm{MHz}, \mathrm{CDCl}_{3}\right) \delta 8.49(\mathrm{~s}, 1 \mathrm{H}), 7.98$ (d, $\left.J=9 \mathrm{~Hz}, 1 \mathrm{H}\right), 7.81$ (d, $\left.J=9 \mathrm{~Hz}, 1 \mathrm{H}\right), 7.70$ (d, $J=9 \mathrm{~Hz}, 1 \mathrm{H}), 7.23(\mathrm{~d}, J=9 \mathrm{~Hz}, 1 \mathrm{H}), 7.17(\mathrm{~s}, 1 \mathrm{H}), 6.62(\mathrm{~s}, 2 \mathrm{H}), 6.44(\mathrm{~s}, 1 \mathrm{H}), 5.09(\mathrm{~s}, 2 \mathrm{H})$, $4.09(\mathrm{t}, J=4.8 \mathrm{~Hz}, 4 \mathrm{H}), 3.93(\mathrm{~s}, 3 \mathrm{H}), 3.81(\mathrm{t}, J=4.8 \mathrm{~Hz}, 4 \mathrm{H}), 3.72-3.51(\mathrm{~m}, 24 \mathrm{H}), 2.09-2.06$ $(\mathrm{m}, 6 \mathrm{H}), 1.75-1.70(\mathrm{~m}, 12 \mathrm{H}), 1.65-1.52(\mathrm{~m}, 12 \mathrm{H}) .{ }^{13} \mathrm{C} \mathrm{NMR}\left(75 \mathrm{MHz}, \mathrm{CDCl}_{3}\right) \delta 167.5$, $160.4,158.7,138.9,137.2,131.1,130.9,128.2,127.1,126.1,125.5,120.0,107.3,106.2$, $101.3,72.3,71.4,71.3,71.0,70.8,70.2,69.8,67.7,60.5,59.3,52.3,47.7,36.6,30.7 . \mathrm{MS}$ (ESP) calcd for $\mathrm{C}_{55} \mathrm{H}_{76} \mathrm{O}_{13}$ (M) 945.1; found 945.4.

A solution of the methyl ester of 2 in THF $(20 \mathrm{ml})$ was slowly added to a solution of excess $\mathrm{NaOMe}$ (freshly prepared) in $\mathrm{MeOH}(20 \mathrm{ml})$. The mixture was refluxed overnight, the solvent was removed, and the residue was acidified with $\mathrm{HCl}(\mathrm{aq})$ and extracted with EtOAc. The evaporation of solvent gave compound 2 (quantitative yield) ${ }^{1} \mathrm{H} \operatorname{NMR}\left(300 \mathrm{MHz}, \mathrm{CDCl}_{3}\right) \delta 8.54(\mathrm{~s}, 1 \mathrm{H}), 8.00(\mathrm{~d}, J=9 \mathrm{~Hz}, 1 \mathrm{H}), 7.80(\mathrm{~d}, J=9 \mathrm{~Hz}, 1 \mathrm{H}), 7.68$ (d, $J=9 \mathrm{~Hz}, 1 \mathrm{H}), 7.21(\mathrm{~d}, J=9 \mathrm{~Hz}, 1 \mathrm{H}), 7.14(\mathrm{~s}, 1 \mathrm{H}), 6.59(\mathrm{~s}, 2 \mathrm{H}), 6.43(\mathrm{~s}, 1 \mathrm{H}), 5.04(\mathrm{~s}, 2 \mathrm{H})$, $4.07(\mathrm{t}, J=4.8 \mathrm{~Hz}, 4 \mathrm{H}), 3.80$ (t, $J=4.8 \mathrm{~Hz}, 4 \mathrm{H}), 3.70-3.52$ (m, 24H), 2.13-2.04 (m, 6H), 1.74$1.69(\mathrm{~m}, 12 \mathrm{H}), 1.65-1.50(\mathrm{~m}, 12 \mathrm{H}) .{ }^{13} \mathrm{C} \mathrm{NMR}\left(75 \mathrm{MHz}, \mathrm{CDCl}_{3}\right) \delta 170.9,160.3,158.9,138.9$, $137.5,131.7,131.2,128.1,127.1,126.3,125.0,120.0,107.3,106.2,101.3,72.5,71.4,71.2$, 70.9, 70.8, 70.1, 69.8, 67.7, 60.5, 59.3, 41.6, 36.6, 30.7. MS (ESP) calcd for $\mathrm{C}_{54} \mathrm{H}_{74} \mathrm{O}_{13}(\mathrm{M})$ 931.1; found 931.4.

EDTA-based tetraethylene glycol dimer 3. A solution of compound $\mathbf{1}(0.10 \mathrm{~g}, 0.14 \mathrm{mmol})$ in $50 \%$ trifluoroacetic acid- $\mathrm{CH}_{2} \mathrm{Cl}_{2}(10 \mathrm{~mL})$ was stirred overnight at rt. Solvents were removed under reduced pressure followed by the addition and then evaporation of $\mathrm{CH}_{2} \mathrm{Cl}_{2}$ 
$(3 \times 10 \mathrm{~mL})$ and finally diethyl ether $(10 \mathrm{~mL})$. The residue was taken up in $\mathrm{H}_{2} \mathrm{O}$, filtered, and lyophilized to give 3 (quantitative yield).

${ }^{1} \mathrm{H}$ NMR (300 MHz, $\left.\mathrm{CDCl}_{3}\right) \delta$ 3.81-3.40 (m, 32H), 3.32-3.27 (m, 4H), 3.12-3.08 (m, 8H). MS (ESP) calcd for $\mathrm{C}_{43} \mathrm{H}_{68} \mathrm{O}_{11}(\mathrm{M}+\mathrm{H})$ 761.5; found 761.4.

$\mathbf{E u}^{3+}$ complex of 3 . The Eu(III) complex of $\mathbf{3}$ was synthesized similar to $\mathbf{1} \cdot \mathrm{Eu}^{3+}$. MS (ESP) calcd for $\mathrm{C}_{26} \mathrm{H}_{48} \mathrm{EuN}_{4} \mathrm{O}_{14}(\mathrm{M}+\mathrm{H})$ 793.64; found 793.2.

\section{1-Naphthalene sulfonic acid-6-(3,5-di(tetraethylene glycol adamantyl ether) benzyl ether}

4. $\mathrm{Na}(46.6 \mathrm{mg}, 2.02 \mathrm{mmol})$ was dissolved in $\mathrm{MeOH}$ previously cooled in an ice-bath. 6Hydroxy-2-naphthalene sulfonic acid sodium salt $(0.498 \mathrm{~g}, 2.02 \mathrm{mmol})$ was added and the mixture was stirred and heated to $60{ }^{\circ} \mathrm{C}$ to dissolve any precipitated material. Compound 6 was added with small portions over a period of $15 \mathrm{~min}$. The mixture was refluxed overnight, cooled to room temperature and the solvent was evaporated. The residue was dissolved in chloroform and filtered. The solvent was evaporated and washed several times with diethylether. The product was obtained as a white solid (0.6 g, 30\%)

${ }^{1} \mathrm{H}$ NMR $\left(300 \mathrm{MHz}, \mathrm{CDCl}_{3}\right) \delta 8.29(\mathrm{~s}, 1 \mathrm{H}), 7.81(\mathrm{~d}, J=9 \mathrm{~Hz}, 1 \mathrm{H}), 7.51(\mathrm{~d}, J=9 \mathrm{~Hz}, 1 \mathrm{H}), 7.31$ (d, $J=9 \mathrm{~Hz}, 1 \mathrm{H}), 7.01(\mathrm{~d}, J=9 \mathrm{~Hz}, 1 \mathrm{H}), 6.91(\mathrm{~s}, 1 \mathrm{H}), 6.57$ (s, 2H), 6.43 (s, 1H), $4.94(\mathrm{~s}, 2 \mathrm{H})$, $4.07(\mathrm{~m}, 4 \mathrm{H}), 3.76(\mathrm{~m}, 4 \mathrm{H}), 3.70-3.52(\mathrm{~m}, 24 \mathrm{H}), 2.12-2.01(\mathrm{~m}, 6 \mathrm{H}), 1.72-1.64(\mathrm{~m}, 12 \mathrm{H})$, 1.61-1.46 (m, 12H). MS (ESP) calcd for $\mathrm{C}_{53} \mathrm{H}_{73} \mathrm{NaO}_{14} \mathrm{~S}(\mathrm{M}+\mathrm{H}-\mathrm{Na})$ 967.19; found 967.5.

3,5-bis(tetraethylene glycol adamantyl ether) benzyl alcohol 5. A suspension of bromoethyl triethylene glycol adamantyl ether (1.5 g, $3.83 \mathrm{mmol})$, 3,5-dihydroxy benzyl alcohol (268.5 mg, $1.916 \mathrm{mmol})$, dried potassium carbonate (529.76 mg, $3.83 \mathrm{mmol})$, and 18crown-6 $(0.11 \mathrm{~g}, 0.42 \mathrm{mmol})$ in acetone $(50 \mathrm{~mL})$ was refluxed for $72 \mathrm{~h}$. The solvent was 
evaporated and the residue was partitioned between water $(50 \mathrm{~mL})$ and diethyl ether $(50 \mathrm{~mL})$. The aqueous layer was extracted with diethyl ether $(3 \times 25 \mathrm{~mL})$ and the combined extracts were dried over $\mathrm{MgSO}_{4}$. The solvent was evaporated and the residue was purified by column chromatography (EtOAc, eluent) to give 5 as a colorless oil ( $874.8 \mathrm{mg}, 60 \%)$

${ }^{1} \mathrm{H}$ NMR $\left(300 \mathrm{MHz}, \mathrm{CDCl}_{3}\right) \delta 6.50(\mathrm{~s}, 2 \mathrm{H}), 6.35(\mathrm{~s}, 1 \mathrm{H}), 4.55(\mathrm{~s}, 2 \mathrm{H}), 4.06(\mathrm{t}, J=4.8 \mathrm{~Hz}, 4 \mathrm{H})$, $3.79(\mathrm{t}, J=4.8 \mathrm{~Hz}, 4 \mathrm{H}), 3.69-3.53(\mathrm{~m}, 24 \mathrm{H}), 2.12-2.08(\mathrm{~m}, 6 \mathrm{H}), 1.72-1.68(\mathrm{~m}, 12 \mathrm{H}), 1.64-1.50$ (m, 12H). ${ }^{13} \mathrm{C}$ NMR $\left(75 \mathrm{MHz}, \mathrm{CDCl}_{3}\right) \delta 160.2,143.7,105.7,101.4,72.4,71.4,71.3,71.0$, 70.9, 69.9, 67.7, 65.3, 60.5, 59.3, 41.6, 36.6, 30.6. MS (ESP) calcd for $\mathrm{C}_{43} \mathrm{H}_{68} \mathrm{O}_{11}(\mathrm{M}+\mathrm{H})$ 761.5; found 761.4.

3,5-bis(tetraethylene glycol adamantyl ether) benzyl bromide 6. A solution of phosphorus tribromide $(78.65 \mathrm{mg}, 0.29 \mathrm{mmol})$ in toluene $(5 \mathrm{~mL})$ was added dropwise to a cooled $\left(0{ }^{\circ} \mathrm{C}\right)$ solution of $5(562.0 \mathrm{mg}, 0.73 \mathrm{mmol})$ in toluene $(50 \mathrm{~mL})$. The mixture was stirred for $1 \mathrm{~h}$ at room temperature. The solvent was removed under reduced pressure and the residue was partitioned between dichloromethane $(100 \mathrm{~mL})$ and water $(100 \mathrm{~mL})$. The organic layer was washed with water $(3 \times 50 \mathrm{~mL})$ and brine $(1 \times 50 \mathrm{~mL})$ and dried over $\mathrm{MgSO}_{4}$. The solvent was removed under reduced pressure and the residue was purified by column chromatography (EtOAc, eluent) to give 6 as a colorless oil (456 mg, 75\%).

${ }^{1} \mathrm{H}$ NMR $\left(300 \mathrm{MHz}, \mathrm{CDCl}_{3}\right) \delta 6.52(\mathrm{~s}, 2 \mathrm{H}), 6.40(\mathrm{~s}, 1 \mathrm{H}), 4.36(\mathrm{~s}, 2 \mathrm{H}), 4.07(\mathrm{t}, J=4.8 \mathrm{~Hz}, 4 \mathrm{H})$, $3.81(\mathrm{t}, J=4.8 \mathrm{~Hz}, 4 \mathrm{H}), 3.73-3.50(\mathrm{~m}, 24 \mathrm{H}), 2.15-2.10(\mathrm{~m}, 6 \mathrm{H}), 1.76-1.71(\mathrm{~m}, 12 \mathrm{H}), 1.67-1.53$ (m, 12H). ${ }^{13} \mathrm{C}$ NMR (75 MHz, $\left.\mathrm{CDCl}_{3}\right) \delta 160.2,139.8,128.4,108.1,101.9,72.4,71.6,71.5$, 71.4, 71.3, 71.0, 70.8, 69.8, 67.7, 59.4, 41.6, 36.6, 30.7. MS (ESP) calcd for $\mathrm{C}_{43} \mathrm{H}_{67} \mathrm{BrO}_{10}(\mathrm{M})$ 823.8; found 823.2. 
Substrate and monolayer preparation. Microscope glass slides were used for $\beta$ cyclodextrin $(\beta$-CD) monolayer preparation. The substrates were cleaned with piranha solution for 15 min (concentrated $\mathrm{H}_{2} \mathrm{SO}_{4}$ and $33 \%$ aqueous $\mathrm{H}_{2} \mathrm{O}_{2}$ in a $3: 1$ ratio; Caution: piranha should be handled carefully) and rinsed with MilliQ. After drying in a nitrogen stream, the substrates were used immediately for silanized monolayer formation. The substrates were enclosed in a low-vacuum desiccator with $0.1 \mathrm{ml}$ TPEDA, continually pumping for 5 min to create a vapor phase of TPEDA. After overnight incubation, the slides were rinsed with ethanol and dichloromethane to remove any excess of silanes and subsequently dried in a nitrogen stream. The attachment of 1,4-phenylene diisothiocyanate was made in a $20 \mathrm{mM}$ solution in toluene at $60^{\circ} \mathrm{C}$ during $2 \mathrm{~h}$. Samples were thoroughly rinsed with toluene and dried in a nitrogen flow. The $\beta$-CD layer attachment was made during $2 \mathrm{~h}$ in an aqueous $0.1 \mathrm{mM} \beta$-cyclodextrin-heptaamine solution $(\mathrm{pH} \sim 7)$ at $60^{\circ} \mathrm{C}$. Samples were thoroughly rinsed with water and dried in a nitrogen flow.

Microcontact printing. Patterned silicon substrates were made by photolithography followed by reactive ion etching (RIE) or e-beam lithography. They consisted of gratings of $50 \mu \mathrm{m}$ dots at $70 \mu \mathrm{m}$ period with the height of $1 \mu \mathrm{m}$. PDMS stamps were prepared by casting a 10:1 (v/v) mixture of poly(dimethylsiloxane) (PDMS) prepolymer and curing agent (Sylgard 184, Dow Corning) against a silicon master. After overnight curing at $60{ }^{\circ} \mathrm{C}$, the stamps were oxidized by oxygen plasma for $1 \mathrm{~min}$ and subsequently inked by dropping aqueous adsorbate solution onto the stamp. Before printing, the stamps were blown dried in a stream of nitrogen. The stamps were brought into conformal contact with the substrate for 30 min. The stamps were changed for each new print and the same inking procedure was used. After stamp removal, the printed substrates were rinsed with copious amounts of water, blown dry with nitrogen and imaged with fluorescence microscopy. 
Printing of 1 and 2 (or 4), followed by $\mathbf{E u C l}_{3}$ immersion. The $\beta$-CD SAM substrate was printed for 30 min with a stamp inked with an equimolar ratio of 1 and 2 (or 4) (2 $\mathrm{mM}$ each) in saturated $\beta-\mathrm{CD}$ in a 3:1 mixture of $\mathrm{EtOH} /$ water, followed by rinsing with water and drying in a stream of nitrogen. The printed substrate was immersed in a $4 \mathrm{mM}$ aqueous solution of $\mathrm{EuCl}_{3}$ for $30 \mathrm{~min}$, followed by rinsing and drying in a stream of nitrogen.

Printing of 2 , followed by $\mathbf{1} \cdot \mathbf{E u}^{3+}$ (or $3 \cdot \mathbf{E u}^{3+}$ ) immersion. The $\beta$-CD SAM substrate was printed for 30 min with a stamp inked with a solution of 2 in saturated $\beta-C D$ in a 3:1 mixture of EtOH/water, followed by rinsing with water and drying in a stream of nitrogen. The printed substrate was immersed in a $2 \mathrm{mM}$ aqueous solution of $1 \cdot \mathrm{Eu}^{3+}\left(\right.$ or $3 \cdot \mathrm{Eu}^{3+}$ ) for $30 \mathrm{~min}$, followed by rinsing and drying in a stream of nitrogen.

Printing of different ratios of 1 and 2, followed by $\mathbf{E u C l}_{3}$ immersion. The $\beta$-CD SAM substrate was printed for 30 min with a stamp inked with different molar ratio of 2 and 1 varying from $0 \%: 100 \%, 20 \%: 80 \%, 40 \%: 60 \%, \quad 50 \%: 50 \%, 60 \%: 40 \%, 80 \%: 20 \%$, and $100 \%: 0 \%$ in saturated $\beta-\mathrm{CD}$ in a 3:1 mixture of $\mathrm{EtOH} /$ water, followed by rinsing with water and drying in a stream of nitrogen. The printed substrate was immersed in a $4 \mathrm{mM}$ aqueous solution of $\mathrm{EuCl}_{3}$ for $30 \mathrm{~min}$, followed by rinsing and drying in a stream of nitrogen.

Time-resolved fluorescence measurements. Two sets of samples were prepared with or without $1 \cdot \mathrm{Eu}^{3+}$ present. The first sample was printed with a flat stamp inked with $2 \mathrm{mM}$ of 2 for 30 min onto a $\beta$-CD SAM with saturated $\beta-\mathrm{CD}$ in $3: 1 \mathrm{EtOH} /$ water. The same procedure was used to prepare substrates with an equimolar ratio of 2 and $1 \cdot \mathrm{Eu}^{3+}(2 \mathrm{mM}$ each). In order to enhance the total emission intensity, multiple slides were used in a cuvette during the measurement in air ( 6 double side printed slides in total). The samples were positioned at $45^{\circ}$ 
orientation relative to the excitation light and the detector. The decay curves at $370 \mathrm{~nm}$ for the donor alone (2) and in the presence of the acceptor $\left(1 \cdot \mathrm{Eu}^{3+}\right)$ were measured upon excitation at $282 \mathrm{~nm}$.

\section{Fluorescence microscopy}

Fluorescence microscope images were taken using an Olympus inverted research microscope IX71 equipped with a mercury burner U-RFL-T as light source and a digital Olympus DR70 camera for image acquisition. UV excitation (300 nm $\left.\leq \lambda_{\mathrm{ex}} \leq 400 \mathrm{~nm}\right)$ and blue emission (410 $\mathrm{nm} \leq \lambda_{\mathrm{em}} \leq 510 \mathrm{~nm}$ ) was filtered using a Dapi Olympus filter cube. UV excitation (300 $\mathrm{nm} \leq \lambda_{\text {ex }} \leq 400 \mathrm{~nm}$ ) and red emission (narrow band pass at $615 \mathrm{~nm}$ ) was filtered using a Olympus filter cube. All fluorescence microscopy images were acquired in air.

\section{Fluorescence spectral microscopy}

To record local emission spectra a custom built microscopy setup capable of spectral imaging was used. The sample was illuminated using a mercury lamp and a standard filter cube for UV excitation (excitation $300 \mathrm{~nm}$ to $400 \mathrm{~nm}$, detection $>400 \mathrm{~nm}$ ) via a 100x objective $(1.3$ NA, Olympus). The local emission was collected by the same objective. The emitted light was imaged via a pinhole and a prism spectrometer onto a cooled CCD camera (Newton EMCCD, Andor). Wavelength calibration was achieved using a calibrated light source (Cal2000 Mercury Argon Calibration source, Ocean Optics, USA). 


\section{Fluorescence lifetime spectrophotometry}

Fluorescence lifetimes were determined using a spectrophotometer (FluoroMax4, Horiba Jobin Yvon), equipped with a TCSPC extension and a pulsed $282 \mathrm{~nm}$ NanoLED for excitation (all Horiba Jobin Yvon). The recorded data was analyzed using the DAS6 software package of Horiba Jobin Yvon.

\subsection{References}

[1] a) D. N. Reinhoudt, M. Crego-Calama, Science 2002, 295, 2403-2407; b) V. E. Campbell, J. R. Nitschke, Synlett 2008, 2008, 3077-3090; c) C. A. M. Bradley J. Holliday, Angew. Chem. Int. Ed. 2001, 40, 2022-2043; d) J.-M. Lehn, Chem. Soc. Rev 2007, 36, 151-160; e) J.-M. Lehn, Proc. Natl. Acad. Sci. USA 2002, 99, 4763-4768 ; f)

G. M. Whitesides, R. F. Ismagilov, Science 1999, 284, 89-92; g) R. F. Ludlow, S. Otto, Chem. Soc. Rev. 2008, 37, 101-108; h) B. C. Gibb, Nat. Chem. 2009, 1, 17-18.

[2] A. Langner, S. L. Tait, N. Lin, C. Rajadurai, M. Ruben, K. Kern, Proc. Natl. Acad. Sci. USA 2007, 104 17927-17930

[3] A. Mulder, J. Huskens, D. N. Reinhoudt, Org. Biomol. Chem. 2004, 2, 3409-3424.

[4] M. J. W. Ludden, D. N. Reinhoudt, J. Huskens, Chem. Soc. Rev 2006, 35, 1122-1134.

[5] M. Eigen, The Hypercycle: A Principle of Natural Self Organization, Springer-Verlag 1979.

[6] a) O. Crespo-Biel, C. W. Lim, B. J. Ravoo, D. N. Reinhoudt, J. Huskens, J. Am. Chem. Soc 2006, 128, 17024-17032; b) M. L. W. Ludden, A. Mulder, K. Schulze, V. Subramaniam, R. Tampe, J. Huskens, Chem. Eur. J. 2008, 14, 2044-2051.

[7] C. W. Lim, O. Crespo-Biel, M. C. A. Stuart, D. N. Reinhoudt, J. Huskens, B. J. Ravoo, Proc. Natl. Acad. Sci. USA 2007, 104, 6986-6991.

[8] M. F. Hazenkamp, G. Blasse, N. Sabbatini, J. Phys. Chem. 1991, 95, 783-787. 
[9] G. E. Buonocore, H. Li, B. Marciniak, Coord. Chem. Rev. 1990, 99, 55-87.

[10] D. L. Dexter, J. Chem. Phys. 1953, 21, 836-850.

[11] The surface was consecutively imaged by fluorescence microscopy using two different filter sets: one set B allows UV excitation (300 $\left.\mathrm{nm} \leq \lambda_{\mathrm{ex}} \leq 400 \mathrm{~nm}\right)$ and blue emission (410 $\left.\mathrm{nm} \leq \lambda_{\mathrm{em}} \leq 510 \mathrm{~nm}\right)$ and another set $\mathbf{R}$ allows $\mathrm{UV}$ excitation and red emission (narrow band pass at $615 \mathrm{~nm}$ ).

[12] J. J. Michels, J. Huskens, D. N. Reinhoudt, J. Am. Chem. Soc 2002, 124, 2056-2064.

[13] a) E. Delgado-Pinar, J. C. Frias, L. J. Jimenez-Borreguero, M. T. Albelda, J. Alarcon, E. Garcia-Espana, Chem. Commun. 2007, 3392-3394; b) D. J. Lewis, T. M. Day, J. V. MacPherson, Z. Pikramenou, Chem. Commun. 2006, 1433-1435.

[14] a) J. Massue, S. J. Quinn, T. Gunnlaugsson, J. Am. Chem. Soc. 2008, 130, 6900-6901; b) K. L. Ai, B. H. Zhang, L. H. Lu, Angew. Chem. Int. Ed. 2009, 48, 304-308; c) B. I. Ipe, K. Yoosaf, K. G. Thomas, J. Am. Chem. Soc. 2006, 128, 1907-1913.

[15] J. P. Leonard, C. M. G. dos Santos, S. E. Plush, T. McCabe, T. Gunnlaugsson, Chem. Commun. 2007, 129-131.

[16] P. Luisi, Fundam.Chem. 2002, 4, 1572-8463.

[17] T. Auletta, B. Dordi, A. Mulder, A. Sartori, S. Onclin, C. M. Bruinink, M. Peter, C. A. Nijhuis, H. Beijleveld, H. Schonherr, G. J. Vancso, A. Casnati, R. Ungaro, B. J. Ravoo, J. Huskens, D. N. Reinhoudt, Angew. Chem. Int. Ed. 2004, 43, 369-373. 


\section{Chapter 4}

\section{Ratiometric Fluorescent Detection of an Anthrax Biomarker at Molecular Printboards*}

A novel surface-assisted fluorescent sensing system has been developed for the ratiometric detection of an anthrax biomarker (dipicolinic acid, DPA) on a molecular printboard. The system affords a nanomolar sensitivity and high selectivity towards DPA.

* Part of this chapter has been published in: M. Deniz Yilmaz, Shu-Han Hsu, David N. Reinhoudt, Aldrik H. Velders, Jurriaan Huskens, Angew. Chem. Int. Ed. 2010, 49, 59385941. 


\subsection{Introduction}

Anthrax is an acute disease, concurrently a potential biological warfare agent caused by Bacillus Anthracis. The accurate, rapid, sensitive and selective detection of Bacillus spores plays a vital role in order to prevent a biological attack or outbreak of disease. ${ }^{[1]}$ Bacterial spores contain a main core cell which is enclosed by protective layers. As a major component of these protective layers, bacterial spores contain up to $1 \mathrm{M}$ dipicolinic acid (DPA), accounting for $5-15 \%$ of the dry mass of the bacterial spore. ${ }^{[2]}$ Hence, DPA is a convenient biomarker for these spores.

In recent years a number of biological and chemical detection methods for Bacillus Anthracis spores have been investigated. Biological methods are based on polymerase chain reactions $^{[3]}$ and immunoassays. ${ }^{[4]}$ Important chemical methods employ vibrational spectroscopy (FT-IR, raman and SERS) ${ }^{[5]}$ and photoluminescence. ${ }^{[6]}$ Among them, lanthanide $\left(\mathrm{Ln}^{3+}\right)$-based luminescent detection of DPA has been most promising owing to the unique photophysical properties of $\mathrm{Ln}^{3+}$-DPA chelates including their bright luminescence upon sensitization by DPA, the long luminescence lifetimes compared to free $\mathrm{Ln}^{3+}$, and the concomitantly high luminescence enhancement ratio upon coordination of DPA to the $\mathrm{Ln}^{3+}$ center. ${ }^{[7]}$ Besides the use of DPA itself as a sensitizer, ratiometric fluorescent detection of anthrax spores can be achieved through the displacement of a different sensitizer by DPA.

Molecular recognition processes at monolayers on surfaces offer advantages compared to solution-based sensing such as a fast response time, minimization of analyte sorption time to the receptor, and real-time and real-space measurements. ${ }^{[8]}$ Glass is an appropriate substrate for fluorescence detection of chemical species owing to its transparency, inertness to light and easy modification with a monolayer of organic adsorbates. ${ }^{[9]}$ Microarrays on glass allow for rapid, simultaneous, and multiple analyte sensing on glass slides. Previous studies have indicated that fluorescent monolayers on glass can be employed in the fabrication of microarrays via soft lithography such as microcontact 
printing $(\mu \mathrm{CP})$ which is an efficient and low-cost lithographic technique to create patterned surfaces. Using $\mu \mathrm{CP}$ on glass as a substrate enables the use of fluorescence microscopy for direct visualization of the created fluorescent patterns. ${ }^{[10]}$

Ratiometric detection of chemical species, i.e. the recording of the relative fluorescence intensities at two different wavelengths, has attracted interest owing to an increased accuracy and reproducibility of analyte detection compared to measurements performed at a single wavelength. ${ }^{[11]}$ Strong ratiometric fluorescence responses have been achieved in solid films and fluorescent monolayers as well as in solution. ${ }^{[12]}$

\subsection{Results and discussion}

In the current study we present a novel platform for the ratiometric detection of the Bacillus Anthracis biomarker DPA with high sensitivity and selectivity on a supramolecular monolayer surface. We employ so-called molecular printboards. ${ }^{[13]}$ which are monolayers of $\beta$-cyclodextrin $(\beta-C D)$ on a surface to which building blocks are attached in a noncovalent fashion that allow ratiometric DPA sensing. To the best of our knowledge, this system constitutes the first lanthanide-based surface receptor system for the detection of DPA, as well as the first example of ratiometric DPA detection at a surface.

In Chapter 3 we have demonstrated the surface-assisted sensitized luminescence of $\mathrm{Eu}^{3+}$ on a molecular printboard. ${ }^{[14]}$ Here, we fabricated these luminescent patterns for the ratiometric detection of DPA on a receptor surface, as outlined in Scheme 4.1. Two building blocks have been used in this study: an EDTA-based ligand (1) for binding $\mathrm{Eu}^{3+}$, and a naphthalene-based antenna (2) for coordination to $\mathrm{Eu}^{3+}$ via the carboxylate moiety. Both building blocks have adamantyl groups for immobilization onto the $\beta$-CD monolayer. To fabricate patterned sensing surfaces, a stepwise procedure was followed. Briefly, in the first step, an equimolar mixture of $\mathbf{1}$ and $\mathbf{2}$ was printed onto the $\beta-\mathrm{CD}$ monolayer by $\mu \mathrm{CP}$ to 
generate surface patterns of the ligand pairs. After thorough rinsing with water and drying, the patterned surface was imaged by fluorescence microscopy using filter $\mathbf{B}\left(300<\lambda_{\text {exc }}<400\right.$ $\left.\mathrm{nm}, 410<\lambda_{\mathrm{em}}<510\right)$ and $\mathbf{R}\left(300<\lambda_{\mathrm{exc}}<400 \mathrm{~nm}, \lambda_{\mathrm{em}}=615 \mathrm{~nm}\right)$. As such, filter $\mathbf{B}$ collects the naphthalene emission of $\mathbf{2}$, while filter $\mathbf{R}$ only collects the $\mathrm{Eu}^{3+}$ emission of $1 . \mathrm{Eu}^{3+}$, both with excellent selectivity. Subsequently, the glass slides were immersed in a $\mathrm{EuCl}_{3}$ solution for 30 min to convert 1 into the $1 . \mathrm{Eu}^{3+}$ complex, followed by fluorescence imaging. Subsequently, substrates were incubated into aqueous solutions of DPA with different concentrations $(\mathrm{pH}=6.5)$ for 10 min with continuous stirring. After rinsing and drying, the substrates were again imaged. It is important to note that direct DPA excitation at $270 \mathrm{~nm}$ is inhibited by the narrow band excitation filter at $350 \mathrm{~nm}$.

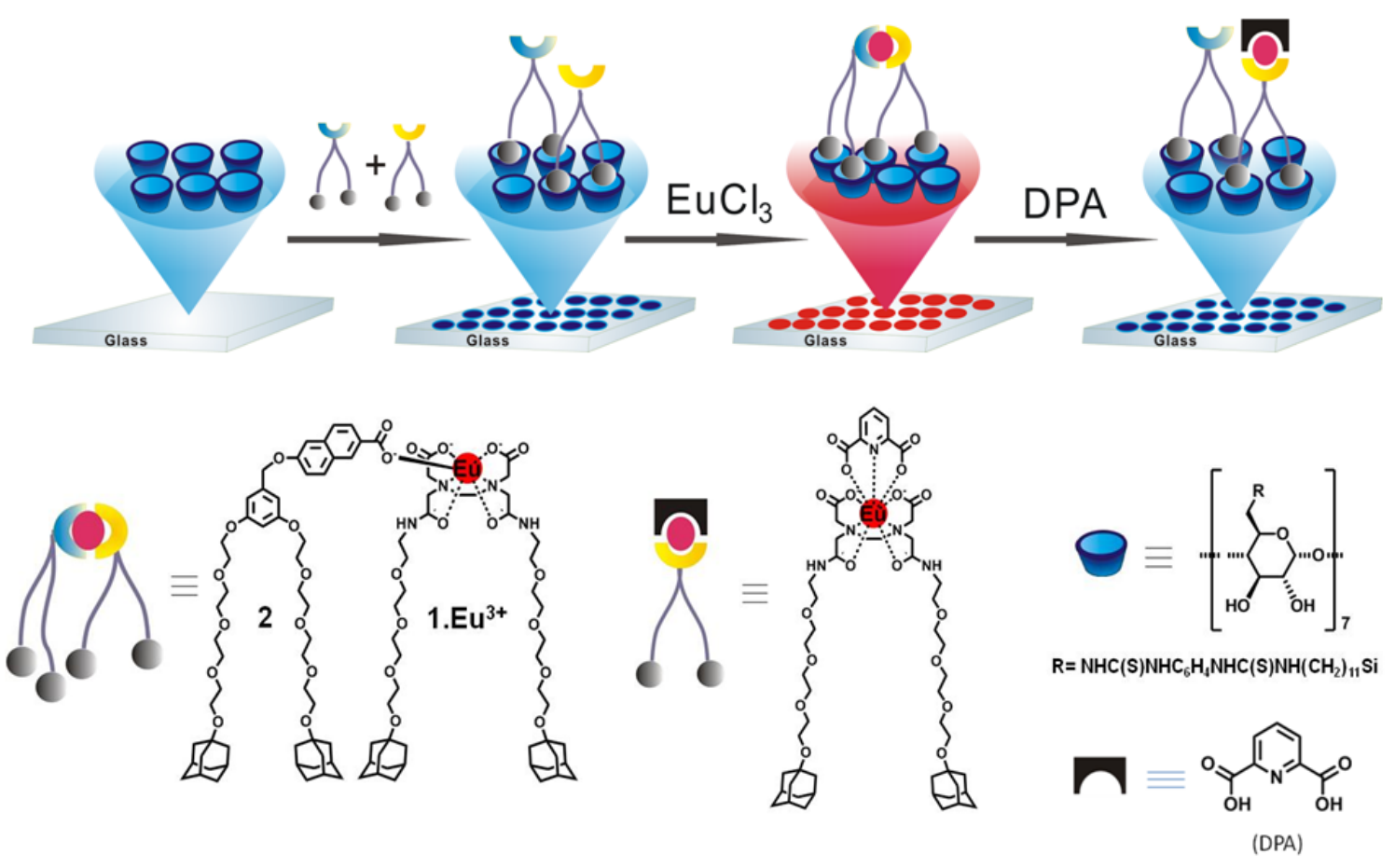

Scheme 4.1 Schematic illustration of the build-up of the supramolecular sensing surface system and the detection of the anthrax biomarker DPA (top), and compounds used in this study (bottom). 
Fluorescence images for all steps are shown in Figure 4.1. In the first step, only the blue naphthalene emission was observed using filter $\mathrm{B}$ and no $\mathrm{Eu}^{3+}$ emission was seen by filter R. After incubation in the $\mathrm{EuCl}_{3}$ solution, the red emission of $\mathrm{Eu}^{3+}$ appeared (filter $\mathrm{R}$ ) and the blue emission of naphthalene decreased (filter B) due to energy transfer from the Eucoordinated naphthalene moiety of 2 to the $\mathrm{Eu}^{3+}$ center of $1 . \mathrm{Eu}^{3+}$, as studied before. ${ }^{[14]}$ In the last step, owing to the displacement of the naphthalene moiety by DPA, the blue emission was recovered and the red emission disappeared.
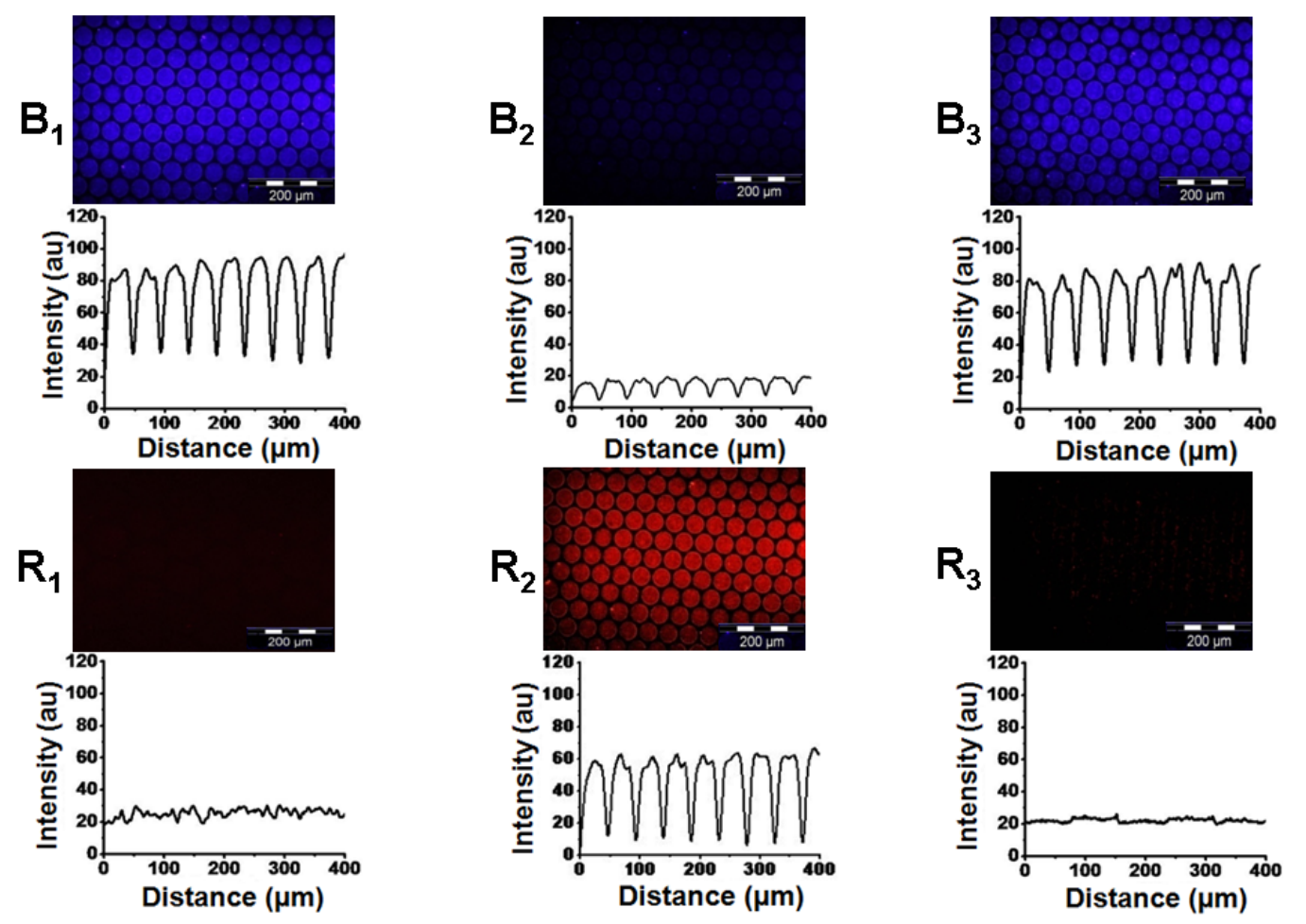

Figure 4.1 Fluorescence microscopy images and corresponding intensity profiles of $50 \mu \mathrm{m}$ dots on $\beta-C D$ monolayers of an equimolar ratio of $\mathbf{1}$ and $2\left(B_{1}, R_{1}\right)$, subsequently incubated in a solution of $\mathrm{EuCl}_{3}$ for $30 \mathrm{~min}\left(\mathrm{~B}_{2}, \mathrm{R}_{2}\right)$, followed by rinsing with MilliQ water and immersion into a $200 \mathrm{nM}$ solution of DPA $\left(\mathrm{B}_{3}, \mathrm{R}_{3}\right)$. 
The fluorescence images clearly indicate that the recovery of the naphthalene emission is practically complete in the presence of only $200 \mathrm{nM}$ DPA. This concentration, corresponding to $7 \times 10^{5}$ spores per $\mathrm{mL}$, is about 3 orders of magnitude lower than the infectious dose of the spores. ${ }^{[7 f, 15]}$ To determine the ratiometric sensing behavior of the DPA binding, values of blue over red emission intensities $\left(\mathrm{I}_{\mathrm{B}} / \mathrm{I}_{\mathrm{R}}\right)$ were plotted for the different samples (Figure 1B).

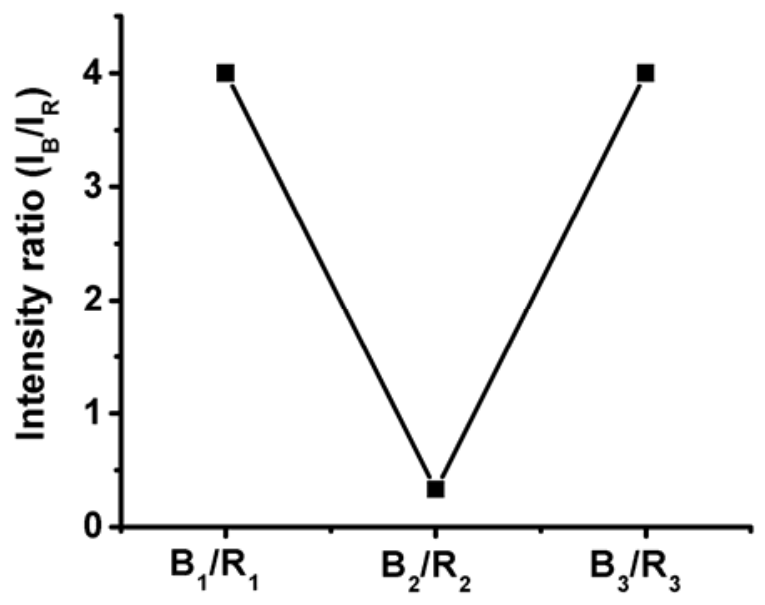

Figure 4.2 Plot of the ratios of the maximum intensities of blue and red images in Figure 4.1 upon $\mathrm{Eu}^{3+}$ and DPA binding.

As a proof of DPA coordination to $\mathrm{Eu}^{3+}$ on the surface, a UV-Vis spectrum of DPA1. $\mathrm{Eu}^{3+}$ on quartz was compared to a solution spectrum of $0.5 \mathrm{mM}$ aqueous DPA-1.Eu ${ }^{3+}$. To avoid the overlap of the naphthalene and DPA absorbances, only $1 . \mathrm{Eu}^{3+}$ was printed on a $\beta$ CD monolayer on quartz, followed by incubation into $1 \mathrm{mM}$ DPA (pH 6.5). The UV-Vis spectrum (Figure 4.2) showed the characteristic broad absorption bands between 260-280 nm as also observed in solution. This confirms that DPA forms a complex with 1.Eu ${ }^{3+}$ on the surface, but also that the large excess of DPA does not remove the $\mathrm{Eu}^{3+}$ from ligand 1, even at high $(\mathrm{mM})$ concentrations. This is attributed to the fact that the stability constant of the 
EDTA-Eu ${ }^{3+}$ complex is nearly 8 orders of magnitude larger than that of the DPA-Eu ${ }^{3+}$ complex in water. ${ }^{[7 a, 16]}$

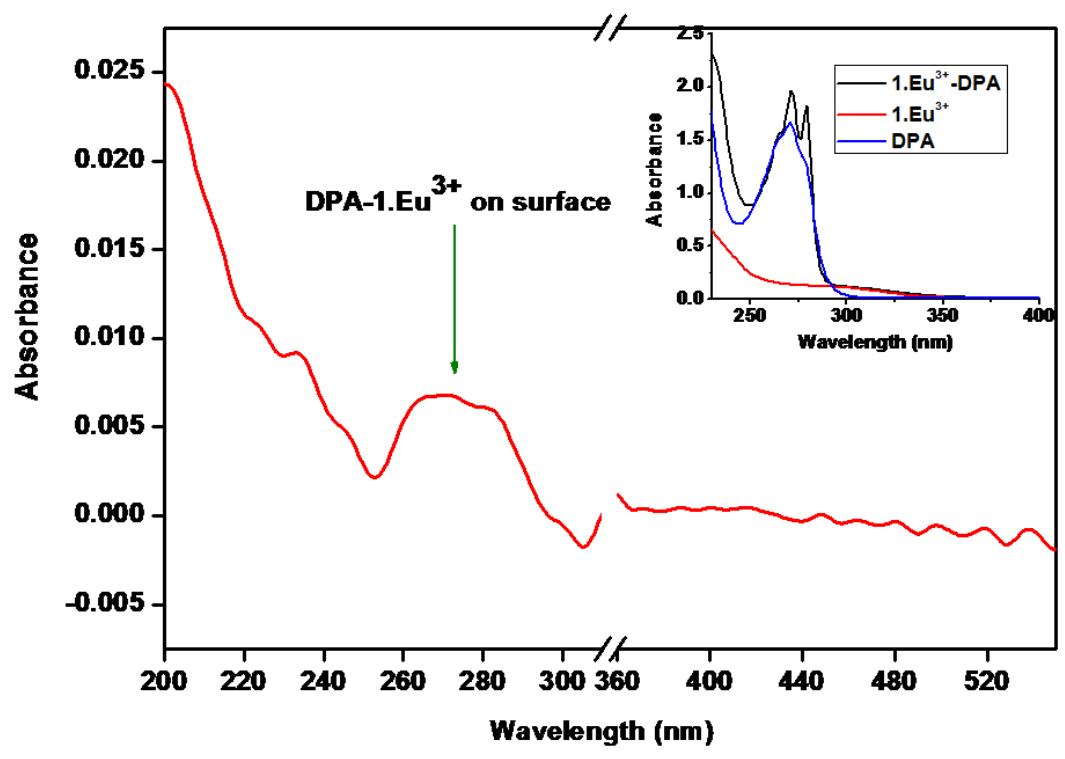

Figure 4.3 UV-Vis spectrum of $1 . \mathrm{Eu}^{3+}$-DPA complex on a $\beta$-CD monolayer on quartz. Inset shows UV-vis spectra of $0.5 \mathrm{mM}$ solutions of DPA, 1.Eu ${ }^{3+}$ and DPA-1.Eu ${ }^{3+}$ in water $(\mathrm{pH}$ $6.5)$.

The fluorescence intensity ratio of the $\mathrm{Eu}^{3+}$-based sensing platform as a function of time upon addition of $200 \mathrm{~nm}$ DPA and corresponding fluorescence images are depicted in Figure 4.4. It clearly indicates that the sensing response is complete within $10 \mathrm{~min}$. 


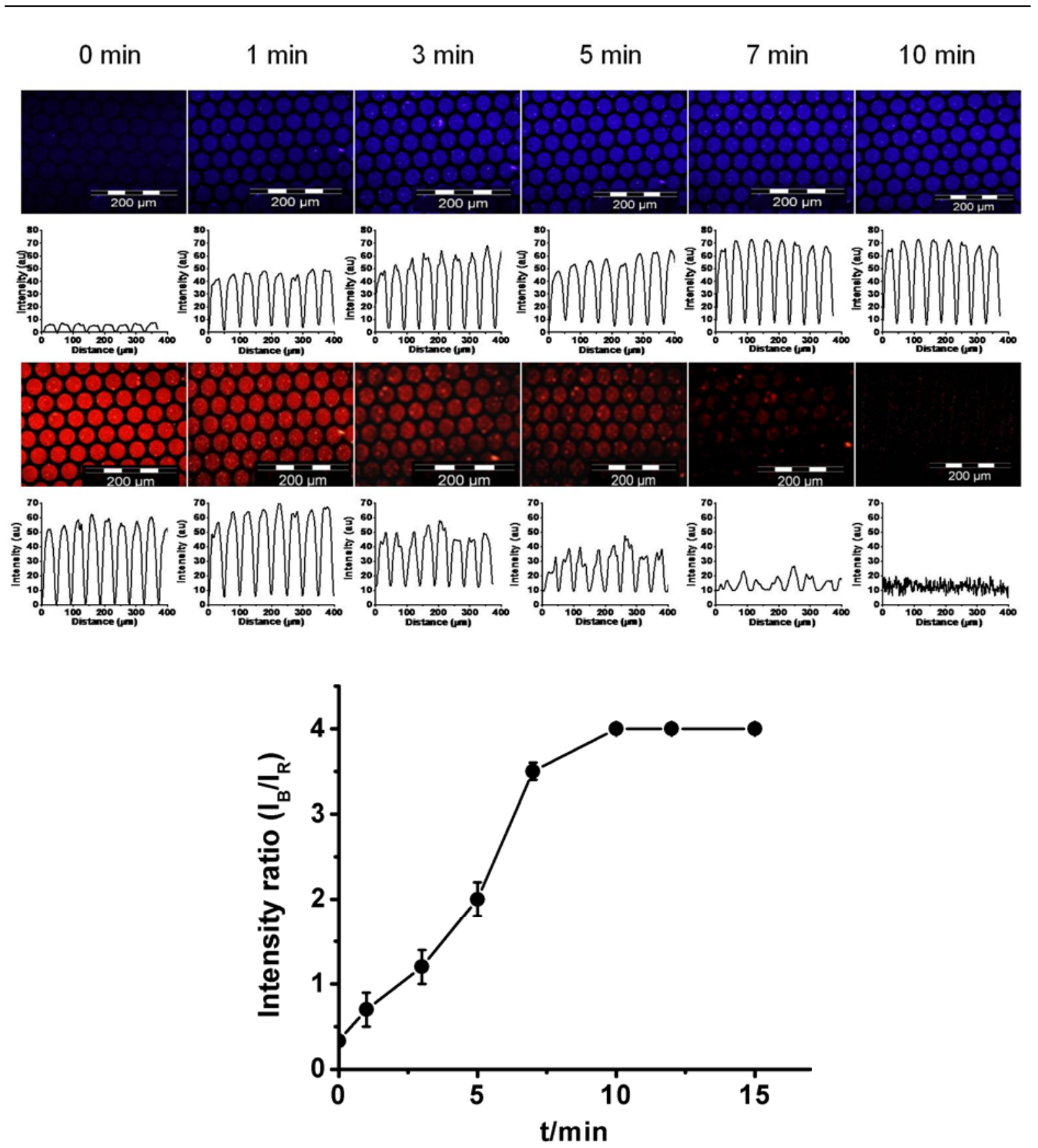

Figure 4.4 Fluorescence microscopy images (top) of $50 \mu \mathrm{m}$ dots prepared on $\beta$-CD monolayers by $\mu \mathrm{CP}(30 \mathrm{~min})$ of an equimolar ratio of $\mathbf{1}$ and $\mathbf{2}$, subsequently incubated in a solution of $\mathrm{EuCl}_{3}$ for 30 min, followed by rinsing with MilliQ water and immersed into a 200 nM solution of DPA in $\mathrm{H}_{2} \mathrm{O}$ ( $\mathrm{pH}$ 6.5) with different incubation times and plot (bottom) of the fluorescence intensity ratios in time. 
To determine the detection limit of DPA, intensity ratios of the detection substrates were plotted against the concentration of DPA (Figure 4.5). Treatment of the surface with relatively high concentrations of DPA resulted in the expected maximal increase of the blue to red ratio, and reached saturation around $200 \mathrm{nM}$ of DPA. However, at lower concentrations of DPA, the ratio was lower, and a detection limit of approx. $25 \mathrm{nM}$ of DPA was obtained.

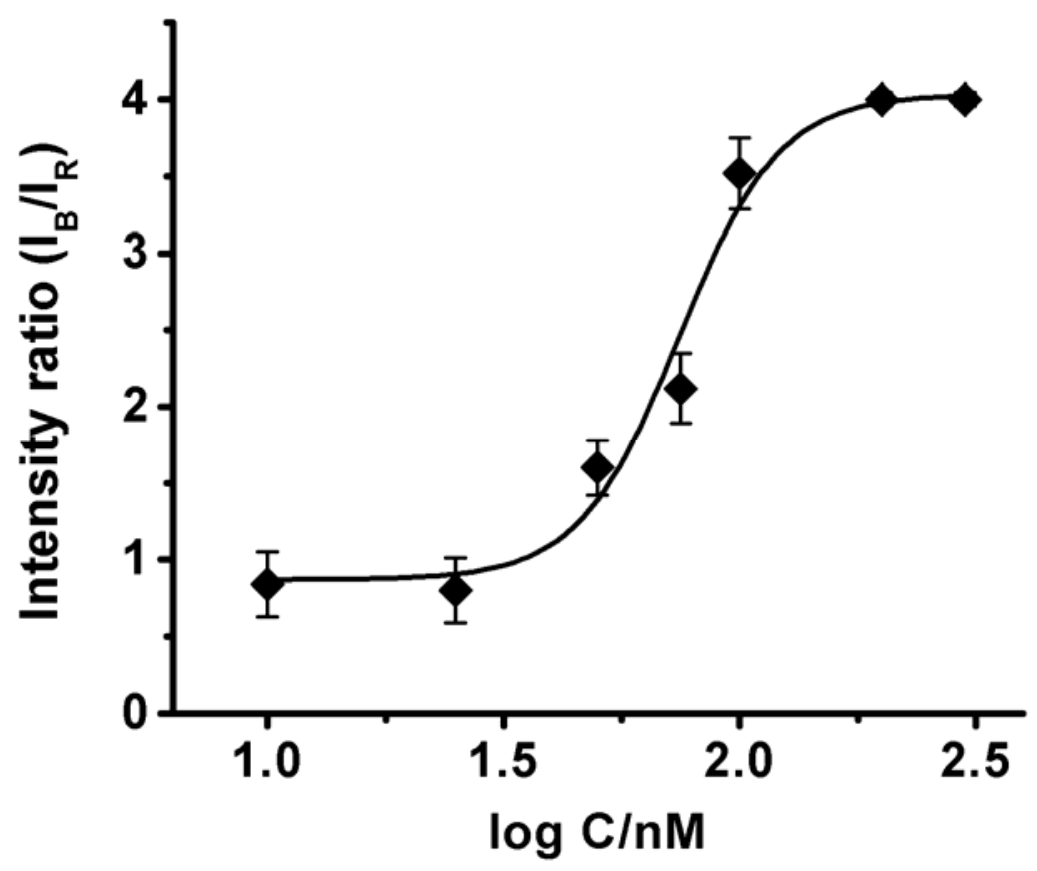

Figure 4.5 Ratiometric fluorescence change of the supramolecular sensing surface as a function of DPA concentration.

To show the selectivity of our system as a sensing platform for DPA, ratiometric fluorescence changes of patterns upon addition of different aromatic ligands such as the $o / m / p$-phthalic acids, nicotinic acid and its two isomers (picolinic and isonicotinic acid), and nicotinamide adenine dinucleotide (NAD) were investigated in $200 \mathrm{nM}$ aqueous solutions at pH 6.5 (Figure 4.6). The small or negligible fluorescence changes, combined with their lower occurrence in the spores, ${ }^{[7 \mathrm{e}]}$ demonstrates the excellent selectivity. 
Dipicolinic Isophthalic Phthalic Picolinic Terephthalic acid acid acid acid
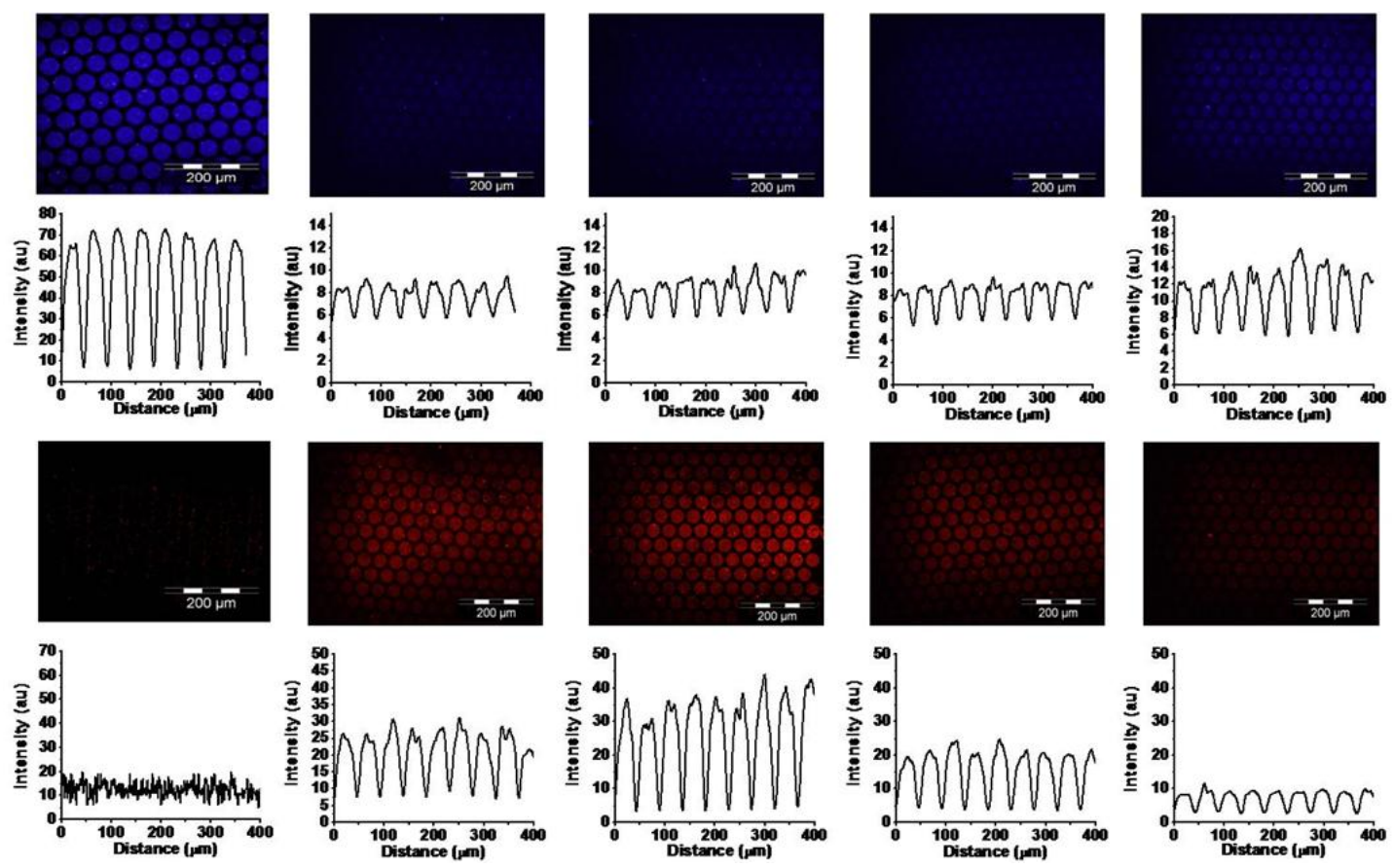

Isonicotinic acid
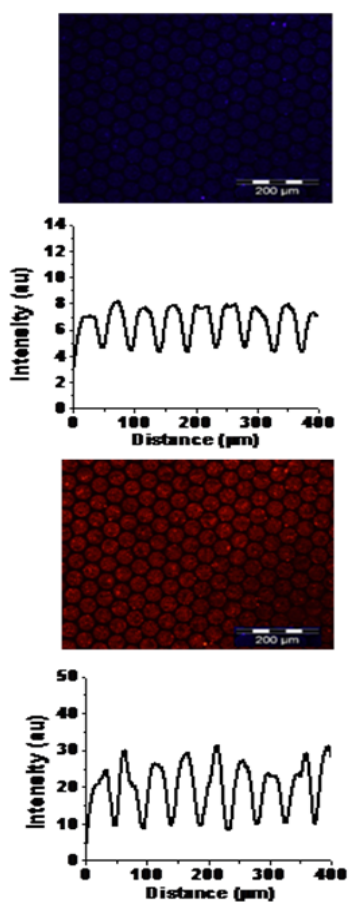

Nicotinamide adenine dinucleotide (NAD)
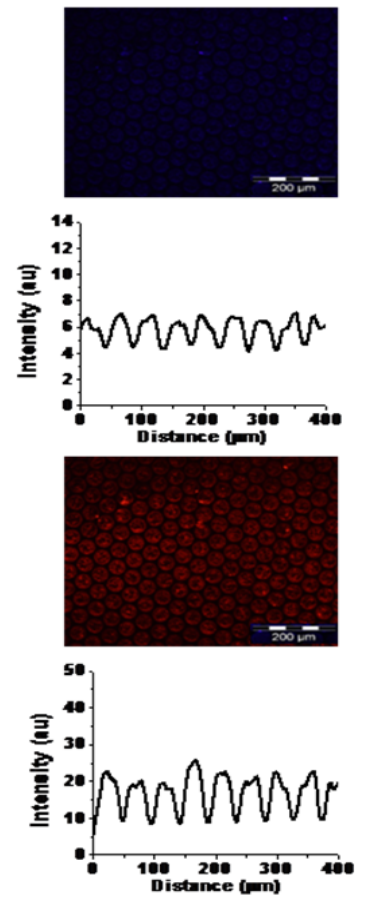

Nicotinic acid
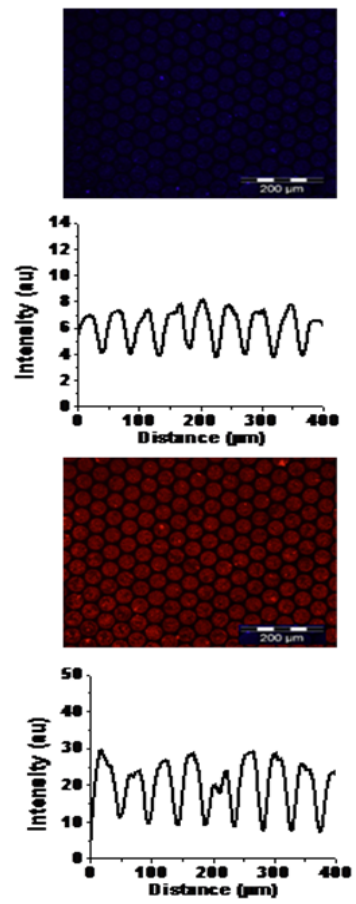


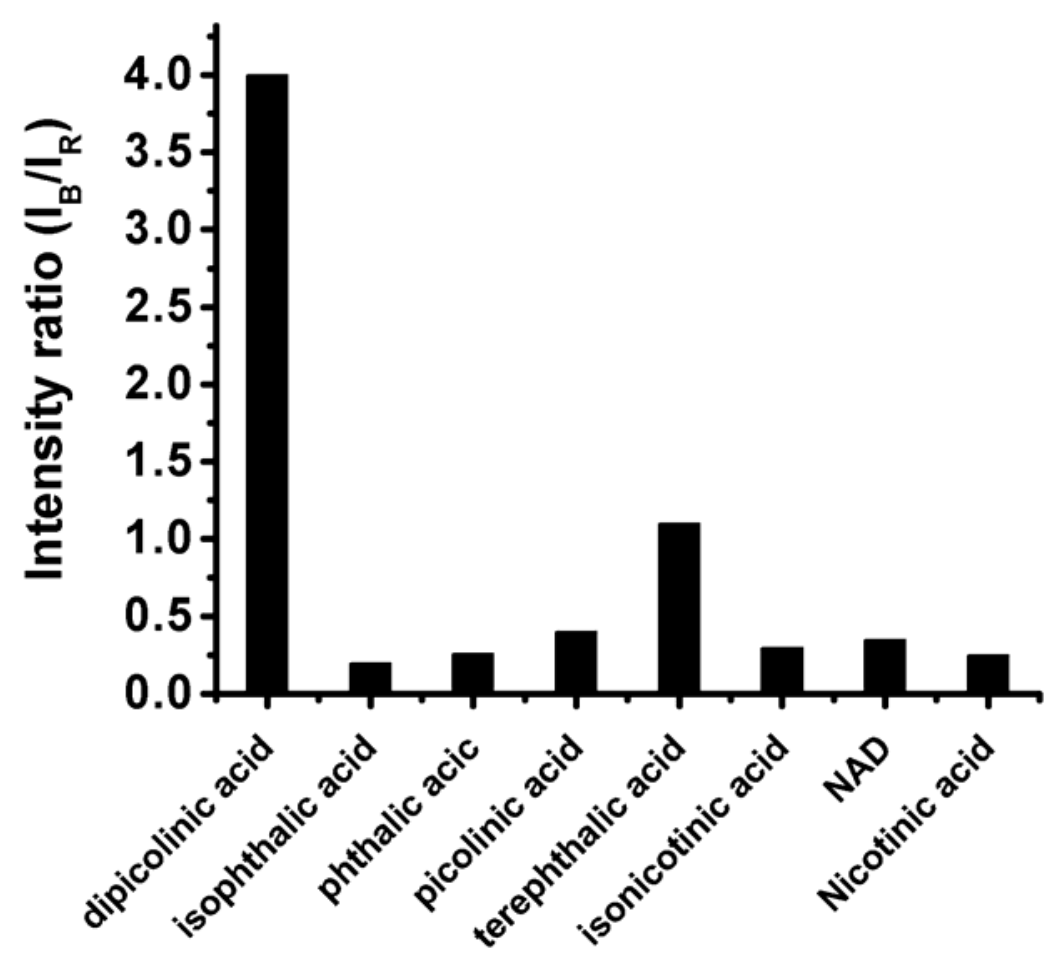

Figure 4.6 Fluorescence images and corresponding bar graph of the supramolecular sensing surface to different competitive aromatic ligands (200 $\mathrm{nM}$ for each ligand).

\subsection{Conclusions}

In conclusion, we have demonstrated that the supramolecular europium-based luminescent sensing platform developed here can be employed for the ratiometric fluorescent detection of the anthrax biomarker DPA on glass substrates. The results show the excellent nanomolar sensitivity of the system toward DPA. Moreover, the system has a remarkable selectivity over other potentially competitive aromatic ligands in water. This surface-assisted sensing system opens new avenues to build solid-state devices for the detection of biologically relevant ions and bacterial spores. From this point of view, real-time monitoring and imaging of analytes on a surface can be extended with supramolecular systems to yield new classes of sensing platforms on surfaces or in microfluidic devices. For creating a 
practical sensor device, more attention needs to be paid to investigating other antenna systems to eliminate the need for UV excitation, shorter fabrication routes, and other possible cross-sensitivities. Overall, the system exemplifies the power of noncovalent strategies to create sensing platforms since different small building blocks can be easily designed and synthesized and subsequently assembled onto such a platform to create a functional system.

\subsection{Acknowledgement}

Shu-Han Hsu is acknowledged for making fruithful discussions.

\subsection{Experimental section}

Materials. N-[3-(trimethoxysilyl)propyl]-ethylenediamine (TPEDA), 1,4-phenylene diisothiocyanate, dipicolinic acid, isophthalic acid, phthalic acid, terephthalic acid, picolinic acid, nicotinic acid, isonicotinic acid, and nicotinamide adenine dinucleotide were used as such. $\beta-\mathrm{CD}$ heptamine was synthesized as described before. ${ }^{[13]}$ Compounds $\mathbf{1}, \mathbf{1 . E u ^ { 3 + }}$ and 2 used in this study were prepared and used as described previously (see Chapter 3). ${ }^{[14]}$

Substrate and monolayer preparation. Microscope glass slides were used for $\beta$ cyclodextrin $(\beta-\mathrm{CD})$ monolayer preparation. ${ }^{[14]}$ The substrates were cleaned with piranha solution for 15 min (concentrated $\mathrm{H}_{2} \mathrm{SO}_{4}$ and $33 \%$ aqueous $\mathrm{H}_{2} \mathrm{O}_{2}$ in a 3:1 ratio; Caution: piranha should be handled carefully) and rinsed with MilliQ. After drying in a nitrogen stream, the substrates were used immediately for silanized monolayer formation. The substrates were enclosed in a low-vacuum desiccator with $0.1 \mathrm{ml}$ TPEDA, continually pumping for 5 min to create a vapor phase of TPEDA. After overnight incubation, the slides were rinsed with ethanol and dichloromethane to remove any excess of silanes and subsequently dried in a nitrogen stream. The attachment of 1,4-phenylene diisothiocyanate 
was made in a $20 \mathrm{mM}$ solution in toluene at $60^{\circ} \mathrm{C}$ during $2 \mathrm{~h}$. Samples were thoroughly rinsed with toluene and dried in a nitrogen flow. The $\beta$-CD layer attachment was made during $2 \mathrm{~h}$ in an aqueous $0.1 \mathrm{mM} \beta$-cyclodextrin-heptaamine solution $(\mathrm{pH} \sim 7)$ at $60^{\circ} \mathrm{C}$. Samples were thoroughly rinsed with water and dried in a nitrogen flow.

Microcontact printing. Patterned silicon substrates were made by photolithography followed by reactive ion etching (RIE) or e-beam lithography. They consisted of gratings of $50 \mu \mathrm{m}$ dots at $70 \mu \mathrm{m}$ period with the height of $1 \mu \mathrm{m}$. PDMS stamps were prepared by casting a 10:1 (v/v) mixture of poly(dimethylsiloxane) (PDMS) prepolymer and curing agent (Sylgard 184, Dow Corning) against a silicon master. After overnight curing at $60{ }^{\circ} \mathrm{C}$, the stamps were oxidized by oxygen plasma for $1 \mathrm{~min}$ and subsequently inked by dropping aqueous adsorbate solution onto the stamp. Before printing, the stamps were blown dried in a stream of nitrogen. The stamps were brought into conformal contact with the substrate for 30 min. The stamps were changed for each new print and the same inking procedure was used. After stamp removal, the printed substrates were rinsed with copious amounts of water, blown dry with nitrogen and imaged with fluorescence microscopy.

Fabrication of the sensing platform and DPA detection: The substrate was printed with an equimolar mixture of 1 and $\mathbf{2}(2 \mathrm{mM}$ each) for $30 \mathrm{~min}$ onto a $\beta$-CD substrate with saturated native $\beta$-CD present in the EtOH/MilliQ mixture (3:1). This was followed by rinsing with MilliQ water and drying in a stream of nitrogen. The printed substrates were immersed in a 4 $\mathrm{mM}$ solution of $\mathrm{EuCl}_{3}$ in MilliQ water for $30 \mathrm{~min}$, followed by rinsing and drying. Afterwards, for concentration-dependent measurements, glass slides were incubated into the solutions of DPA with different concentrations in $\mathrm{H}_{2} \mathrm{O}(\mathrm{pH}=6.5)$ for 10 min with continuous stirring, followed by rinsing with water and drying with nitrogen flow. For time-dependent 
measurements, glass slides were incubated into a $200 \mathrm{nM}$ solution of DPA in $\mathrm{H}_{2} \mathrm{O}(\mathrm{pH}=6.5)$ with different incubation times with continuous stirring, followed by rinsing with water and drying with nitrogen flow. For selectivity measurements, glass slides were incubated into 200 $\mathrm{nM}$ solutions of different competitor aromatic ligands for $10 \mathrm{~min}$ with continuous stirring, followed by rinsing with water and drying with nitrogen flow.

\section{Fluorescence microscopy}

Fluorescence microscope images were taken using an Olympus inverted research microscope IX71 equipped with a mercury burner U-RFL-T as light source and a digital Olympus DR70 camera for image acquisition. UV excitation (300 nm $\left.\leq \lambda_{\text {ex }} \leq 400 \mathrm{~nm}\right)$ and blue emission (410 $\mathrm{nm} \leq \lambda_{\text {em }} \leq 510 \mathrm{~nm}$ ) was filtered using a Dapi Olympus filter cube. UV excitation (300 $\mathrm{nm} \leq \lambda_{\mathrm{ex}} \leq 400 \mathrm{~nm}$ ) and red emission (narrow band pass at $615 \mathrm{~nm}$ ) was filtered using a Olympus filter cube. All fluorescence microscopy images were acquired in air.

\subsection{References}

[1] a) D. A. Henderson, Science 1999, 283, 1279-1282; b) M. Enserink, Science 2001, 294, 1266-1267; c) M. Enserink, Science 2001, 294, 490-491; d) P. T. Yung, E. D. Lester, G. Bearman, A. Ponce, Biotechnol. Bioeng. 2007, 98, 864-871; e) W. T. Sanderson, R. R. Stoddard, A. S. Echt, C. A. Piacitelli, D. Kim, J. Horan, M. M. Davies, R. E. McCleery, P. Muller, T. M. Schnorr, E. M. Ward, T. R. Hales, J. Appl. Microbiol. 2004, 96, 1048-1056; f) R. J. Sharp, A. G. Roberts, J. Chem. Technol. Biot. 2006, 81, 1612-1625; g) M. Zourob, S. Elwary, A. Turner, Principles of 
bacterial detection : biosensors, recognition receptors, and microsystems, Springer, New York, 2008; h) G. Coimbatore, S. M. Presley, J. Boyd, E. J. Marsland, G. P. Cobb in Advances in Biological and Chemical Terrorism Countermeasures, (Eds.: R. J. Kendall, S. M. Presley, G. P. Austin, P. N. Smith), CRC press, FL, 2008, pp. 159175; i) P. A. Lieberzeit, F. L. Dickert, Anal. Bioanal.Chem. 2008, 391, 1629-1639.

[2] a) G. F. Bailey, S. Karp, L. E. Sacks, J. Bacteriol. 1965, 89, 984; b) R. Goodacre, B. Shann, R. J. Gilbert, E. M. Timmins, A. C. McGovern, B. K. Alsberg, D. B. Kell, N. A. Logan, Anal. Chem. 2000, 72, 119-127; c) D. R. Walt, Anal. Chem. 2000, 72, 738a-746a; d) G. W. Gould, A. J. Sale, W. A. Hamilton, J. Gen. Microbiol. 1969, 57, R28; e) L. J. Rode, J. W. Foster, Nature 1960, 188, 1132-1134; f)H. S. Levinson, M. T. Hyatt, J. Bacteriol. 1955, 70, 368-374.

[3] a) W. Hurtle, E. Bode, D. A. Kulesh, R. S. Kaplan, J. Garrison, D. Bridge, M. House, M. S. Frye, B. Loveless, D. Norwood, J. Clin. Microbiol. 2004, 42, 179-185; b) A. Fasanella, S. Losito, R. Adone, F. Ciuchini, T. Trotta, S. A. Altamura, D. Chiocco, G. Ippolito, J. Clin. Microbiol. 2003, 41, 896-899.

[4] a) R. H. Yolken, S. B. Wee, J. Clin. Microbiol. 1984, 19, 356-360; b) D. King, V. Luna, A. Cannons, J. Cattani, P. Amuso, J. Clin. Microbiol. 2003, 41, 3454-3455.

[5] a) G. Thompson, C. Forster, Water Research 2003, 37, 2636-2644; b) R. M. Jarvis, R. Goodacre, Anal. Chem. 2004, 76, 40-47; c) X. Y. Zhang, M. A. Young, O. Lyandres, R. P. Van Duyne, J. Am. Chem. Soc. 2005, 127, 4484-4489.

[6] P. M. Pellegrino, N. F. Fell, J. B. Gillespie, Anal. Chim. Acta 2002, 455, 167-177.

[7] a) J. P. Kirby, M. L. Cable, D. J. Levine, H. B. Gray, A. Ponce, Anal. Chem. 2008, 80, 5750-5754; b) M. L. Cable, J. P. Kirby, K. Sorasaenee, H. B. Gray, A. Ponce, J. Am. Chem. Soc. 2007, 129, 1474-1475; c) E. D. Lester, A. Ponce, IEEE Eng. Med. Biol. 2002, 21, 38-42; d) E. D. Lester, G. Bearman, A. Ponce, IEEE Eng. Med .Biol. 2004, 
23, 130-135; e) Q. Y. Li, P. K. Dasgupta, H. K. Temkin, Environ. Sci. Technol. 2008, 42, 2799-2804; f) K. L. Ai, B. H. Zhang, L. H. Lu, Angew. Chem. Int. Ed. 2009, 48, 304-308; g) M. L. Cable, J. P. Kirby, D. J. Levine, M. J. Manary, H. B. Gray, A. Ponce, J. Am. Chem. Soc. 2009, 131, 9562-9570.

[8] a) R. Zimmerman, L. Basabe-Desmonts, F. van der Baan, D. N. Reinhoudt, M. CregoCalama, J. Mater. Chem. 2005, 15, 2772-2777; b) Y. R. Kim, H. J. Kim, J. S. Kim, H. Kim, Adv. Mat. 2008, 20, 4428-4432.

[9] a) M. Crego-Calama, D. N. Reinhoudt, Adv. Mater. 2001, 13, 1171-1174; b) H. J. Kim, S. J. Lee, S. Y. Park, J. H. Jung, J. S. Kim, Adv. Mater. 2008, 20, 3229-3234.

[10] L. Basabe-Desmonts, J. Beld, R. S. Zimmerman, J. Hernando, P. Mela, M. F. G. Parajo, N. F. van Hulst, A. van den Berg, D. N. Reinhoudt, M. Crego-Calama, J. Am. Chem. Soc. 2004, 126, 7293-7299.

[11] a) M. S. Tremblay, M. Halim, D. Sames, J. Am. Chem. Soc. 2007, 129, 7570-7577; b) H. Takakusa, K. Kikuchi, Y. Urano, H. Kojima, T. Nagano, Chem. Eur. J. 2003, 9, 1479-1485.

[12] J. R. Acharya, H. Zhang, X. Li, E. E. Nesterov, J. Am. Chem. Soc. 2009, 131, 880881.

[13] M. J. W. Ludden, D. N. Reinhoudt, J. Huskens, Chem. Soc. Rev. 2006, 35, 1122.

[14] S. H. Hsu, M. D. Yilmaz, C. Blum, V. Subramaniam, D. N. Reinhoudt, A. H. Velders, J. Huskens, J. Am. Chem. Soc. 2009, 131, 12567-12569.

[15] H. S. Shafaat, A. Ponce, Appl. Environ. Microbiol. 2006, 72, 6808-6814.

[16] S. L. Wu, W. D. Horrocks, Jr., Anal. Chem. 1996, 68, 394-401. 


\section{Chapter 5}

\section{A Supramolecular Sensing Platform in a Microfluidic Chip*}

A supramolecular platform based on self-assembled monolayers (SAMs) has been implemented in a microfluidic device. The system has been applied for the sensing of two different analyte types: biologically relevant phosphate anions and aromatic carboxylic acids which are important for anthrax detection. A Eu(III)-EDTA complex was bound to $\beta$ cyclodextrin monolayers via orthogonal supramolecular host-guest interactions. The selfassembly of the Eu(III)-EDTA conjugate and naphthalene $\beta$-diketone as an antenna resulted in the formation of a highly luminescent lanthanide complex on the microchannel surface. Detection of different phosphate anions and aromatic carboxylic acids was demonstrated by monitoring the decrease in red emission following displacement of the antenna by the analyte. Parallel fabrication of five sensing SAMs in a single multichannel chip was performed, as a first demonstration of phosphate and carboxylic acid screening in a highthroughput format that allows a general detection platform for both analyte systems in a single test run.

* Part of this chapter has been published in: Bilge Eker, M. Deniz Yilmaz, Stefan Schlautmann, J. G. E. Gardeniers, Jurriaan Huskens, submitted. 


\subsection{Introduction}

It is of utmost importance to detect low concentrations of small molecules and solutes in mixtures of complex milieu for toxicology, ${ }^{[1]}$ drug discovery, ${ }^{[2]}$ diagnostics ${ }^{[3]}$ and anti-bioterrorism. ${ }^{[4]}$ Among many chemical, electrochemical, biological, and photoluminescence based small molecule detection, fluorescence turn-on/off sensing has attracted significant attention, offering high sensitivity ${ }^{[5]}$ and reversibility ${ }^{[6]}$ Lanthanide $\left(\mathrm{Ln}^{3+}\right)$ based luminescent detection of small molecules such as nerve agents, ${ }^{[1]}$ antagonists, ${ }^{[4]}$ phosphate anions, ${ }^{[7]}$ and the anthrax biomarker DPA ${ }^{[8]}$ has been most promising among the many turn-on/off sensing methods owing to the unique photophysical properties of $\mathrm{Ln}^{3+}$ antenna chelates with their bright luminescence upon sensitization by an antenna, long luminescence lifetimes compared to free $\mathrm{Ln}^{3+}$, and sharp line-like emission bands at long wavelengths, ${ }^{[9]}$ overcoming autofluorescence and light scattering, and resulting in a high color purity of the emitted light. ${ }^{[10]}$

Surface-confined sensing using self-assembled monolayers (SAMs) offers advantages compared to sensing in solution such as ease and reproducibility of SAMs, ${ }^{[11]}$ fast response times owing to the faster analyte - receptor interaction, and real-time and real-space measurements. ${ }^{[12]}$ Previous studies have shown that SAMs can function as optical sensors when functionalized with fluorescent groups on flat surfaces ${ }^{[13]}$ and in microfluidic systems. ${ }^{[14]}$

Phosphate anions play an important role in various physiological events and they take part in almost all metabolic processes. Among various phosphates, pyrophosphate and ATP are crucial anions for the transfer of genetic information, and metabolic and bioenergetic reactions. ${ }^{[15]}$ Fluorescence based detection of biologically relevant phosphate anions still remains a challenge due to the difficulty of designing binding motifs for anions ${ }^{[16]}$ and achieving an effective fluorescent signal change upon anion binding. ${ }^{[5 \mathrm{~d}, 17]}$ Recently, a $\mathrm{Ln}^{3+}$ 
based displacement assay was employed for phosphate anions on gold nanoparticles in solution $^{[7 b]}$ and on a gold surface. ${ }^{[7 a]}$

Concurrently, some aromatic carboxylic acids have crucial importance in bioterrorism, especially in anthrax detection. ${ }^{[18]}$ Anthrax is an acute disease and a potential biological warfare agent caused by Bacillus Anthracis. The detection of Bacillus spores with rapid response, high selectivity, and sensitivity is very important in order to prevent a bioterroristic attack or outbreak of disease ${ }^{[19]}$ Bacillus spores contain up to $1 \mathrm{M}$ dipicolinic acid (DPA), corresponding to $5-15 \%$ of the dry mass of the bacterial spore, ${ }^{[20]}$ offering a convenient biomarker for anthrax detection. $\mathrm{Ln}^{3+}$ based luminescent detection of DPA has been very promising by using DPA as an antenna itself ${ }^{[8 a, 8 b, 21]}$ or as $\operatorname{Ln}^{3+}$ - DPA chelates, ${ }^{[8 c]}$ allowing fast, highly selective and sensitive detection of bacterial spores. Recently, ratiometric detection of DPA was employed by fabricating microarrays via patterning of a monolayer surface. ${ }^{[8 c]}$

In the current study we present a novel high-throughput platform for a general detection method of biologically relevant phosphate anions and the Bacillus Anthracis biomarker DPA on a supramolecular monolayer surface by using a microfluidic approach. The microfluidic approach has attracted significant attention in the last decades for chemical and biological assays because of faster detection time, low consumption of analyte and reagents, and the possibility of integrated continuous monitoring of analyte solutions ${ }^{[14 \mathrm{~b}, 22]}$ Here, $\beta$-cyclodextrin monolayers known as molecular printboards ${ }^{[23]}$ were employed on a glass-silicon microchip surface and building blocks were attached to the monolayers in a noncovalent fashion that allow phosphate anion and DPA sensing. To the best of our knowledge, this microchip based sensing platform constitutes the first lanthanide-based surface receptor system that provides a general detection platform for two different analyte 
systems, as well as the first example of integration of sensing of phosphate anions and DPA in a microfluidic device.

\subsection{Results and discussion}

\subsubsection{Fabrication of the Sensing Platform and Anion Detection}

Surface-assisted sensitized luminescence of $\mathrm{Eu}^{3+}$ on a molecular printboard was demonstrated in a previous study for the sensing of biological molecules such as the Bacillus Anthracis biomarker DPA (see Chapter 4). ${ }^{[8 c]}$ In the current study, luminescent SAMs on a microchip surface were fabricated to develop a general detection platform of small molecules such as the biologically relevant phosphate anions and aromatic carboxylic acids in a highthroughput format. The sensing platform was developed in a microfluidic device by using the controlled attachment of the building blocks in a supramolecular manner, through multivalent orthogonal linkers. Such a supramolecular surface platform was chosen for this study, since supramolecular interactions combined with multivalency offer high flexibility such as controlled positioning of molecules, fine-tuning of assemblies and their interaction properties, binding strength, binding stoichiometry, binding dynamics, and reversibility. ${ }^{[24]}$ Cyclodextrin $(\beta$-CD)-based host-guest chemistry at the microchip interface was applied to orient the attachment of building blocks of interest to the microchip surface. Microchannels are miniaturized platforms that can be individually accessible and addressable, allowing production of sensing arrays for multianalyte systems within a single device. Thereby, the supramolecular sensing system was implemented on the surface of a multichannel chip as a demonstration of a high-throughput sensing device.

A multichannel chip (15 mm x $25 \mathrm{~mm}$ ) with five parallel channels was designed for generation of the sensing array via the formation of $\beta C D$ SAMs and subsequent attachment of building blocks of interest on each channel surface (Figure 5.1a). Each channel has a width of 
$50 \mu \mathrm{m}$ and equal depth, and the channels are separated by $50 \mu \mathrm{m}$. The channels have independent inlets and they share a common outlet. The chip was fabricated in silicon with a glass cover slip.

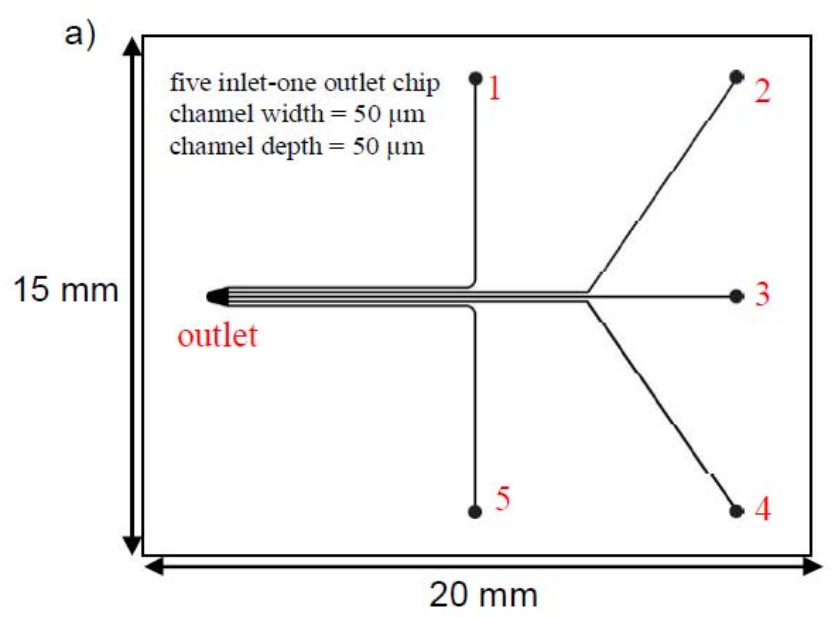

b)
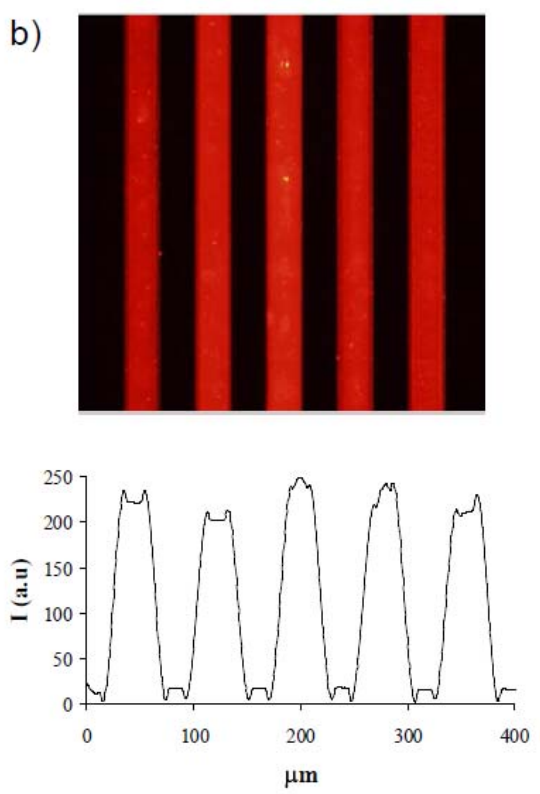

Figure 5.1. Design of the five inlet-one outlet multichannel chip surface (a), and a fluorescence microscopy image and concomitant intensity profile of the red luminescent multichannel device (b).

Parallel synthesis of five sensing SAMs in the multichannel chip was performed by the formation of $\beta \mathrm{CD}$ SAMs on the channel surface in three subsequent steps. ${ }^{[24]}$ The microchip surface was cleaned and activated with piranha, and a amino-terminated TPEDA (N-[3-(trimethoxysilyl)propyl]ethylenediamine) SAM was formed. After DITC $(1,4-$ phenylene-diisothiocyanate) was attached to the TPEDA SAMs, $\beta C D$ heptamine was bound onto the surface in the last step, leading to an array of five $\beta C D$ SAMs confined to a single chip, and providing a functional layer for further surface modification in the multichannel. 
Two building blocks were used to fabricate the sensor surface in the microchip: an ethylenediamine tetraacetic acid (EDTA) - based ligand (1) for binding $\mathrm{Eu}^{3+}$ and the receptor surface, and a naphthalene - based antenna (2) for coordination to $\mathrm{Eu}^{3+}$ via the diketonate moiety (Scheme 5.1). Building block 1 has adamantyl groups for immobilization onto the $\beta C D$ SAMs. To fabricate sensing surfaces on the microchip surface, a stepwise procedure was followed. Briefly, in the first step, $1 \mathrm{mM}$ of $\mathbf{1 . E \mathbf { u } ^ { 3 + }}$ was attached on the $\beta \mathrm{CD}$ monolayer after 30 min incubation in the microchip. After cleaning the microchip surface with water for $10 \mathrm{~min}, 100 \mu \mathrm{M}$ of 2 was incorporated onto $\beta C D$ SAMs via coordination of 2 with $\mathbf{1 . E \mathbf { u } ^ { 3 + }}$ as seen in Scheme 5.1. After a second cleaning step of the microchip surface with water for 10 min, the multichannel surface was imaged by fluorescence microscope using filter $\mathrm{R}$ (300 $\left.<\lambda_{\text {exc }}<400 \mathrm{~nm}, \lambda_{\text {em }}=615 \mathrm{~nm}\right)$. Five luminescent SAM-modified microchannels were visualized simultaneously (Figure $5.1 \mathrm{~b}$ ) by fluorescence microscopy, which showed energy transfer from the Eu-coordinated napthalene moiety of $\mathbf{2}$ to the $\mathrm{Eu}^{3+}$ center of 1.Eu ${ }^{3+}$, and the emission of red light at $615 \mathrm{~nm}$ as studied before. ${ }^{[8 c, 20 \mathrm{~b}]}$ Thereby, a highly luminescent surface platform was achieved in the multichannel device for the detection of phosphates and aromatic carboxylic acids.

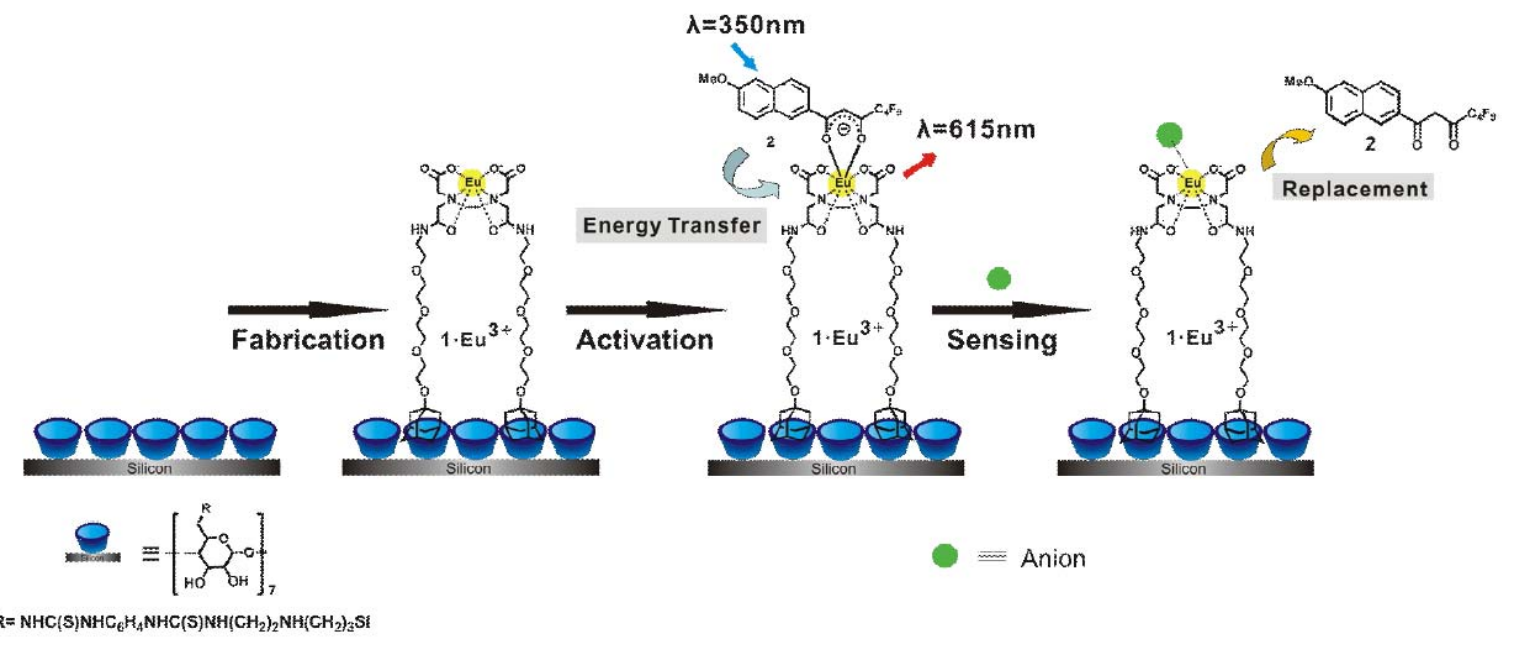

Scheme 5.1. The construction of supramolecular sensing system in a microchip and the detection of an anion of interest. 
The mechanism of anion detection is based on the displacement of 2 from 2-1.Eu ${ }^{3+}$ by a guest anion as shown in Scheme 5.1. Displacement assays have been studied before and the mechanism of displacement is well established, ${ }^{[6 \mathrm{~b}, 25]}$ and the same mechanism is assumed in this study. Briefly, the sensing layer on the microchip surface is an ensemble of 1.Eu $\mathbf{u}^{3+}$ and the antenna 2, and when an anionic guest is added to the ensemble, it displaces 2 , and triggers a fluorescence change upon binding to $\mathbf{1 . E \mathbf { u } ^ { 3 + }}$, leading to a decrease in red fluorescence at $615 \mathrm{~nm}$. Thus, the detection of phosphates and aromatic carboxylic acids is based on the recognition of the guest anion by the $\mathrm{Eu}^{3+}$ - based receptor and the displacement of 2. Thus, the resulting decrease in the fluorescence intensity was monitored and quantified by fluorescence microscopy.

\subsubsection{Sensing of Biologically Relevant Phosphates}

The luminescent multichannel platform was used for the detection of biologically relevant phosphate anions. Five different phosphates (1 mM each), ATP, AMP, ADP, hydrogen phosphate $(\mathrm{Pi})$ and pyrophosphate (PPi) were injected continuously from each inlet (at $1 \mu \mathrm{l} / \mathrm{min}$ by a peristaltic pump) and their sensing was evaluated within $1 \mathrm{~h}$. Among those five phosphate anions, only ATP and PPi caused a strong response at the 2-1.E $\mathbf{u}^{3+}$ complex (Figure 5.2). The sensing of these five anions along with NADP and triphosphate (PPPi) was also studied as a function of time, and around $40 \%$ and $35 \%$ decrease in fluorescence intensity was observed for both ATP and PPPi, while 22\% and 15\% quenching of red emission was observed for PPi, and NADP, respectively, in 10 min $(\mathrm{ATP}>\mathrm{PPPi}>\mathrm{PPi}>$ NADP at $\mathrm{t}=10 \mathrm{~min}$ ) as seen in Figure 5.3. Around $60 \%$ decrease in fluorescence intensity was obtained for ATP after $1 \mathrm{~h}$, whereas PPPi and PPi showed around $45 \%$ decrease in red emission, and the effect of NADP on displacement of the antenna was around $20 \%$ after $1 \mathrm{~h}$ $(\mathrm{ATP}>\mathrm{PPPi} \approx \mathrm{PPi}>\mathrm{NADP}$ at $\mathrm{t}=60 \mathrm{~min})$. The response half-time of ATP was determined based on the exponential decay curve in Figure 5.3, and was found to be about $10 \mathrm{~min}$. 
a)

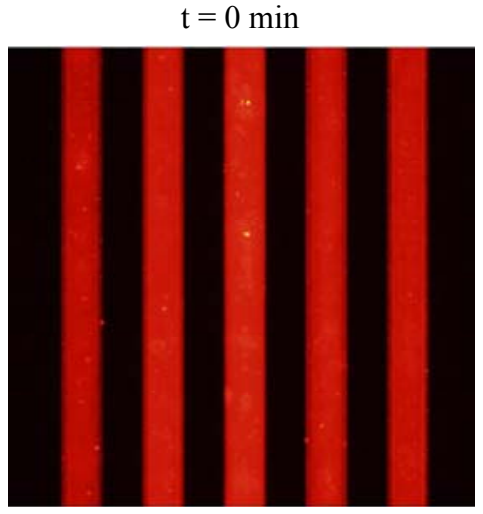

Pi PPi ATP AMP ADP

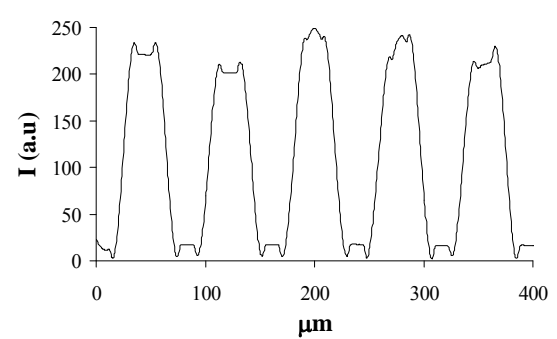

b)

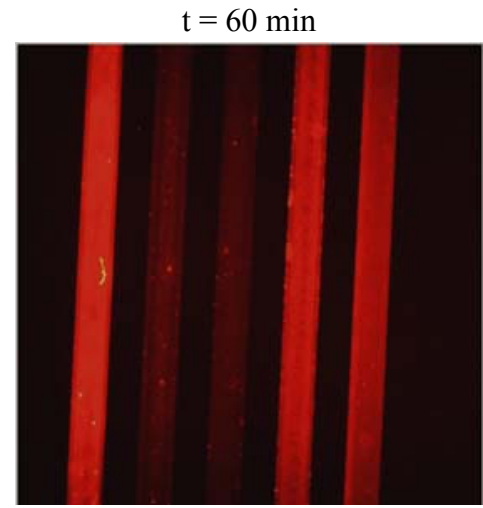

Pi PPi ATP AMP ADP

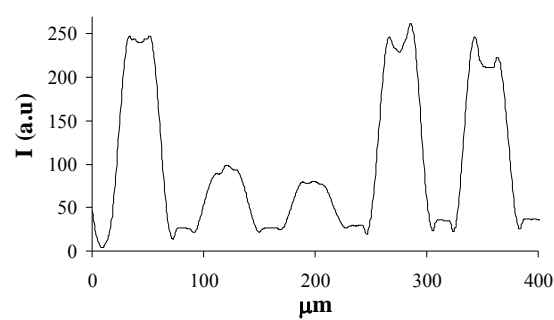

Figure 5.2. The effect of phosphate anions at $1 \mathrm{mM}$ concentration each (from left to right; hydrogen phosphate (Pi), pyrophosphate (PPi), ATP, AMP, ADP) on the displacement of the antenna from 2-1.Eu $\mathbf{u}^{+3}$ on the multichannel surface after 0 min (a) or $60 \mathrm{~min}$ (b), using a flow rate of $1 \mu 1 / \mathrm{min}$.

The reasons behind the different sensing properties of the anions on the microchannel surface are presumably due to differences in binding affinitiy of the anions to $\mathrm{Eu}^{3+}$ which is correlated with the number of phosphate oxygens that coordinate to $\mathrm{Eu}^{3+}$ after the displacement of the antenna. ${ }^{[26]}$ As seen in Figure 5.3, PPPi displaced 2 almost as effectively as ATP indicating the importance of the number of phosphate oxygens on the

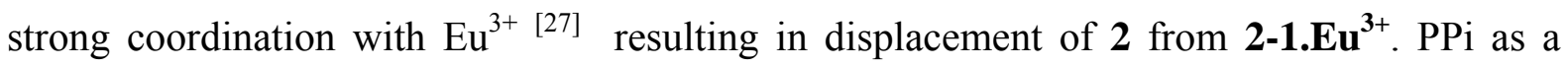
diphosphate also caused a fairly strong response of the $\mathbf{2 - 1 . E \mathbf { u } ^ { 3 + }}$ complex owing to its high binding affinity to $\mathrm{Eu}^{3+} \cdot{ }^{[26]}$ On the other hand, among the diphosphates PPi, ADP, and NADP different responses were seen, especially the sensing of PPi was much stronger than the other 
diphosphates indicating that the side groups of ADP and NADP might decrease the binding affinity of the phosphate groups to $\mathrm{Eu}^{3+}$, thus leading to less effective displacement of $\mathbf{2}$ from 2-1.Eu ${ }^{3+}$ compared to PPi. Thus, these results show that the phosphates possess different sensitivities to the 2-1.E $\mathbf{u}^{3+}$ complex, especially ATP, PPi and PPPi exhibit stronger response to the $\mathrm{Eu}^{3+}$ based assembly compared to the other phosphates. More importantly, this multichannel surface platform allows high-throughput detection of phosphates providing different information for each anion at a single test run.

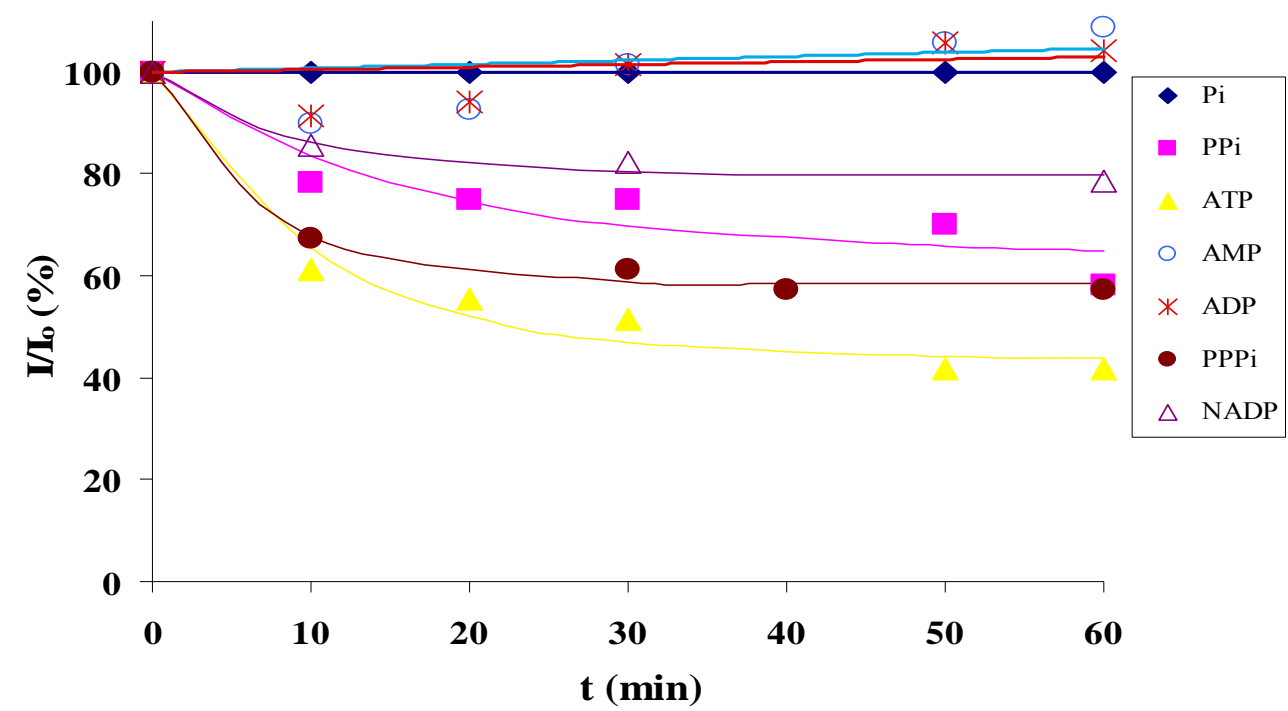

Figure 5.3. Fluorescence intensity vs. time profiles of $1 \mathrm{mM}$ phosphates (Pi, PPi, ATP, AMP, ADP, PPPi, NADP) for selectivity and response time determination on a microchannel surface.

To determine the detection limit of ATP sensing on the microchip platform, ATP detection was performed as a function of concentration. Five different concentrations ranging from $1 \mathrm{mM}$ down to $50 \mu \mathrm{M}$ ATP were injected continuously from each inlet by a peristaltic pump and their sensing was evaluated within $1 \mathrm{~h}$. Figure 5.4a shows the normalized intensity $\left(\mathrm{I} / \mathrm{I}_{0}\right)$ profile of five different concentrations at $\mathrm{t}=60 \mathrm{~min}$. Similar responses were observed with ATP concentrations from $1 \mathrm{mM}$ down to $250 \mu \mathrm{M}$ (around $50 \%-60 \%$ ), and around $35 \%$ decrease in red emission was obtained for $50 \mu \mathrm{M}$ ATP (Figure 5.4b). Thus, ATP sensing 
by the $\mathrm{Eu}^{3+}$ based complex was achieved in the $\mu \mathrm{M}$ range. This is consistent with earlier reported data, which showed ATP detection in the $\mu \mathrm{M},{ }^{[15,28]}$ or $\mathrm{mM}$ concentration range. ${ }^{[29]}$

a)

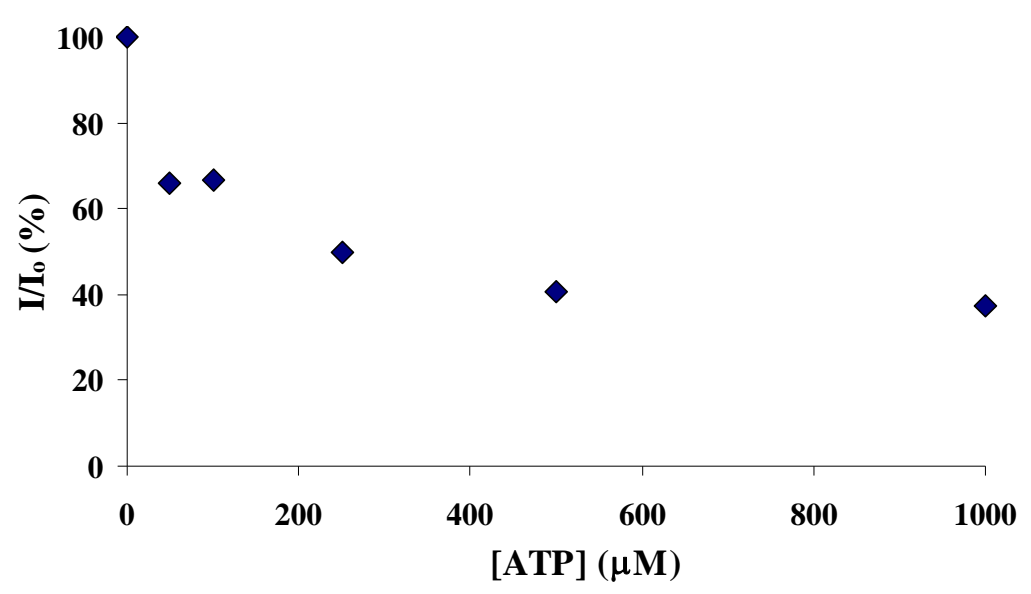

b)

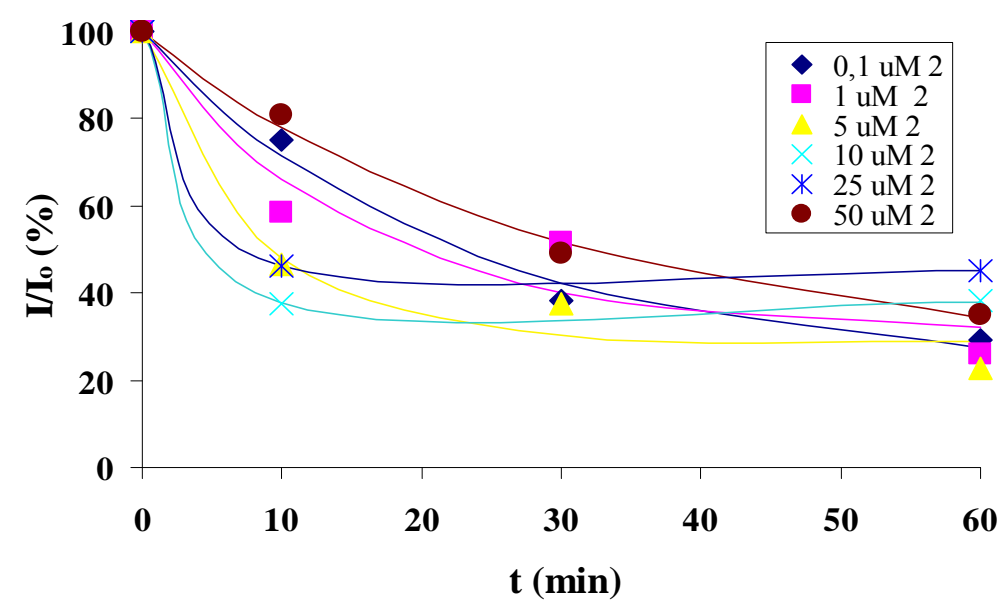

Figure 5.4. The detection sensitivity profile of ATP at $60 \mathrm{~min}$ as a function of different ATP concentrations (a) Fluorescence intensity vs. time profiles of $1 \mathrm{mM}$ ATP in the presence of different concentrations of 2 .

The experiments described above were all performed in the absence of $\mathbf{2}$ in solution, therefore, displacement of $\mathbf{2}$ led to removal of $\mathbf{2}$ from the system and thus to kinetically determined fluorescence readings. Subsequently, ATP sensing was performed in the presence of different concentrations of 2 in order to investigate ATP detection while attempting to reach thermodynamic equilibrium of the displacement reaction of 2 from 2-1.Eu $\mathbf{u}^{3+}$ by ATP. 
Mixtures of $1 \mathrm{mM}$ ATP with five different concentrations of 2, ranging from $0.1 \mu \mathrm{M}$ to 50 $\mu \mathrm{M}$, were injected continuously from each inlet and their sensing of 2-1.Eu $\mathbf{u}^{3+}$ was evaluated within $1 \mathrm{~h}$. Figure 5.4b shows the decrease in red fluorescence was similar for all conditions. No clear trend was obtained for different concentrations of 2, nor in response time. These results show that the binding strength of ATP to $\mathrm{Eu}^{3+}$ is much higher than that of the antenna 2.

\subsubsection{Screening of an Antrax Biomarker and Potentially Interfering Anions}

The same supramolecular lanthanide-based microfluidic platform was also used for the detection of the anthrax biomarker DPA, among other potentially competitive aromatic ligands as a demonstration of a surface-based sensing device that offers a general highthroughput detection platform both for phosphates and aromatic carboxylates. In order to investigate aromatic carboxylic acid detection, five different aromatic carboxylic acids (1 $\mathrm{mM}$ each), $o / m / p$-phthalic acids, picolinic acid and DPA, were injected from each inlet continuously and their sensing was evaluated within $1 \mathrm{~h}$. Among those five aromatic ligands (Figure 5.5a), only DPA was sensed by $\mathbf{2 - 1 . E \mathbf { u } ^ { 3 + }}$ and selectively displaced the antenna, thus quenched around $80 \%$ of the red luminescence in 10 min whereas the effect of the other aromatic acids on the displacement of 2 was negligible (up to $25 \%$ quenching of red emission with terephthalic acid) (Figure 5.5c \& 5.6). Excellent selectivity was achieved with DPA over other potentially interfering aromatic acids after $1 \mathrm{~h}$ (Figure $5.5 \mathrm{~d} \&$ Figure 5.6), while other interferants showed weak response on the sensor surface (up to $35 \%$ quenching of red emission with picolinic and isophthalic acid). The response half-time of DPA was determined based on the exponential decay curve in Figure 5.6, and DPA showed a response half-time of around $5 \mathrm{~min}$.

The high selectivity of DPA sensing on $\mathbf{2 - 1 . E \mathbf { u } ^ { 3 + }}$ is attributed to a higher binding affinity of DPA to $\mathrm{Eu}^{3+[30]}$ due to its stronger coordination to $\mathrm{Eu}^{3+}$ via two carboxylic acid 
groups and the basic nitrogen atom on its aromatic ring, whereas the other aromatic ligands coordinate to $\mathrm{Eu}^{3+}$ via fewer groups. In addition, the carboxylic acid screening in solution was also performed and only DPA selectively displaced 2 after 10 min (data not shown) owing to the much stronger coordination of DPA with $\mathbf{1 . E \mathbf { u } ^ { 3 + }}$ than that of $\mathbf{2}$, showing consistent results with those obtained on receptor surface. Thus, these results show that all aromatic carboxylic acids show different sensing properties to $2-1 . E u^{3+}$ complex, and especially DPA exhibited excellent selectivity and a quick response time (within $10 \mathrm{~min}$ ). Thus, this multichannel sensing platform offers a common detection system for both aromatic carboxylic acids and phosphates.

To determine the detection limit of DPA sensing on the microchip platform, DPA detection was performed as a function of concentration. Initially, five different concentrations ranging from $1 \mathrm{mM}$ down to $500 \mathrm{nM}$ DPA were injected continuously from each inlet by a peristaltic pump and their sensing was evaluated within $1 \mathrm{~h}$. Lower concentrations down to $40 \mathrm{nM}$ were also used to determine the detection limit of DPA. The intensity ratio $\left(\mathrm{I} / \mathrm{I}_{0}\right)$ values of the sensor substrates were plotted as a function of the DPA concentration $(0-1$ $\mu \mathrm{M})$ at $\mathrm{t}=60 \mathrm{~min}$ in Figure 5.7. Lower concentrations of DPA resulted in less quenching of the red emission showing that DPA sensing is concentration dependent. $1 \mu \mathrm{M}$ DPA quenched red emission as effective as $1 \mathrm{mM}$ DPA (around $70 \%-80 \%$ ), and $\mathrm{nM}$ concentrations of DPA still showed significant response on the sensor surface (Figure 5.7). Thus, DPA was sensed by the $\mathrm{Eu}^{3+}$ based complex with $<100 \mathrm{nM}$ detection limit. Such a detection sensitivity range is consistent with literature ${ }^{[9 a]}$.

In a recent study, ratiometric detection of DPA was employed by us at molecular printboards using a lanthanide-based surface-receptor system. ${ }^{[8 c]}$ DPA showed a very high selectivity over other potentially competitive aromatic ligands in water with nanomolar sensitivity, and the sensing response was complete within 10 min on glass substrate, showing 
consistent and similar results to those reported here. Our study demonstrates the development of a microfluidic device by using a different antenna 2 for the detection of bacterial spores and other anions such as biologically relevant phosphates at a high-throughput format.

a)

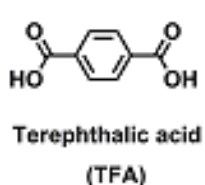

(TFA)

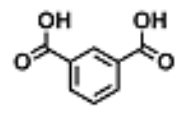

Isophthalic acid

(IPA)

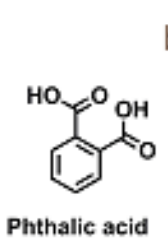

(PA)

b)
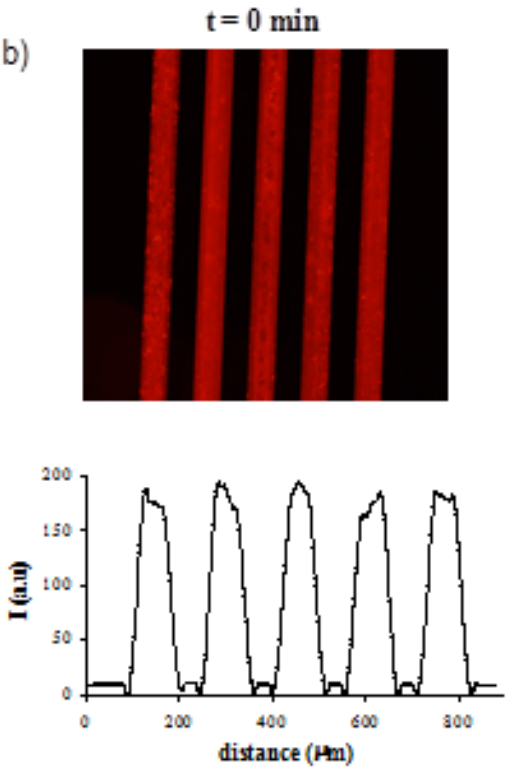

c)

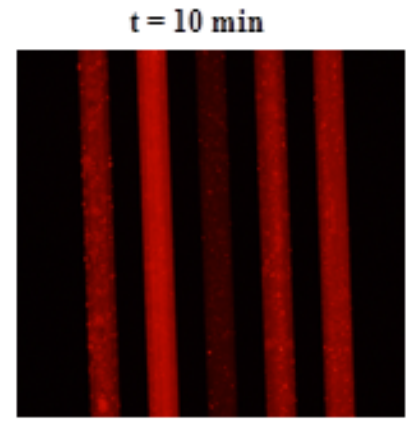

TPA PicA DPA PA IPA

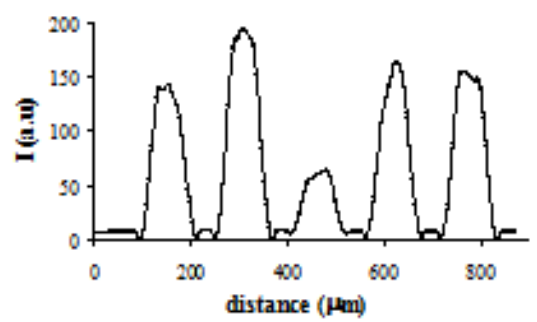

d)

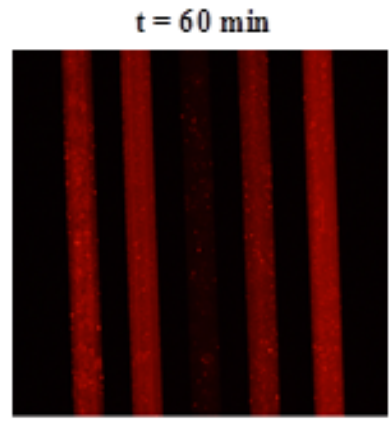

TPA PicA DPA PA IPA

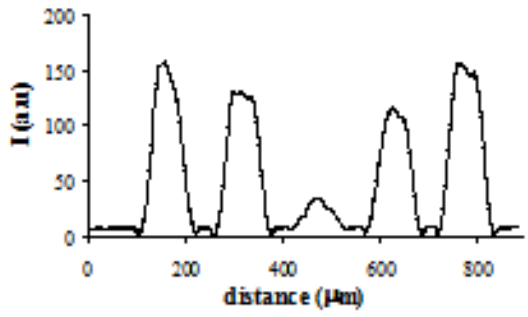

Figure 5.5. The structure of aromatic carboxylic acids: $p$-phthalic acid (TPA), picolinic acid (PicA), dipicolinic acid (DPA), o-phthalic acid (PA), and $m$-phthalic acid (IPA) (a) Fluorescence microscopy images and intensity profiles of the multichannel surface upon carboxylic acid screening at $\mathrm{t}=0 \mathrm{~min}(\mathrm{~b}) \mathrm{t}=10 \min (\mathrm{c})$ and $\mathrm{t}=60 \mathrm{~min}(\mathrm{~d})$. 


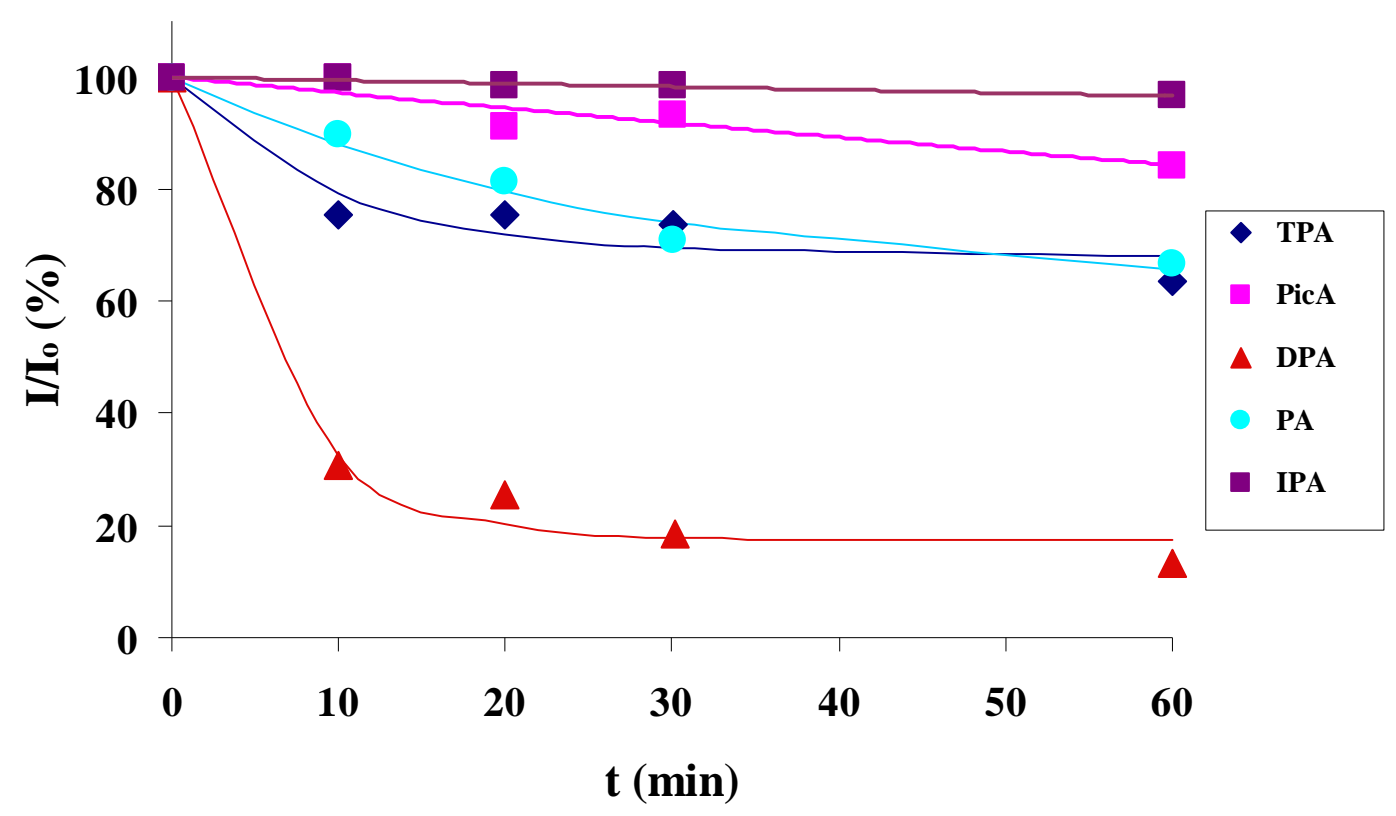

Figure 5.6. Fluorescence Intensity vs. time profiles of $1 \mathrm{mM}$ aromatic carboxylic acids ( $p$ phthalic acid, picolinic acid, DPA, $o$-phthalic acid, and $m$-phthalic acid)) for selectivity and response time determination on the microchannel surface.

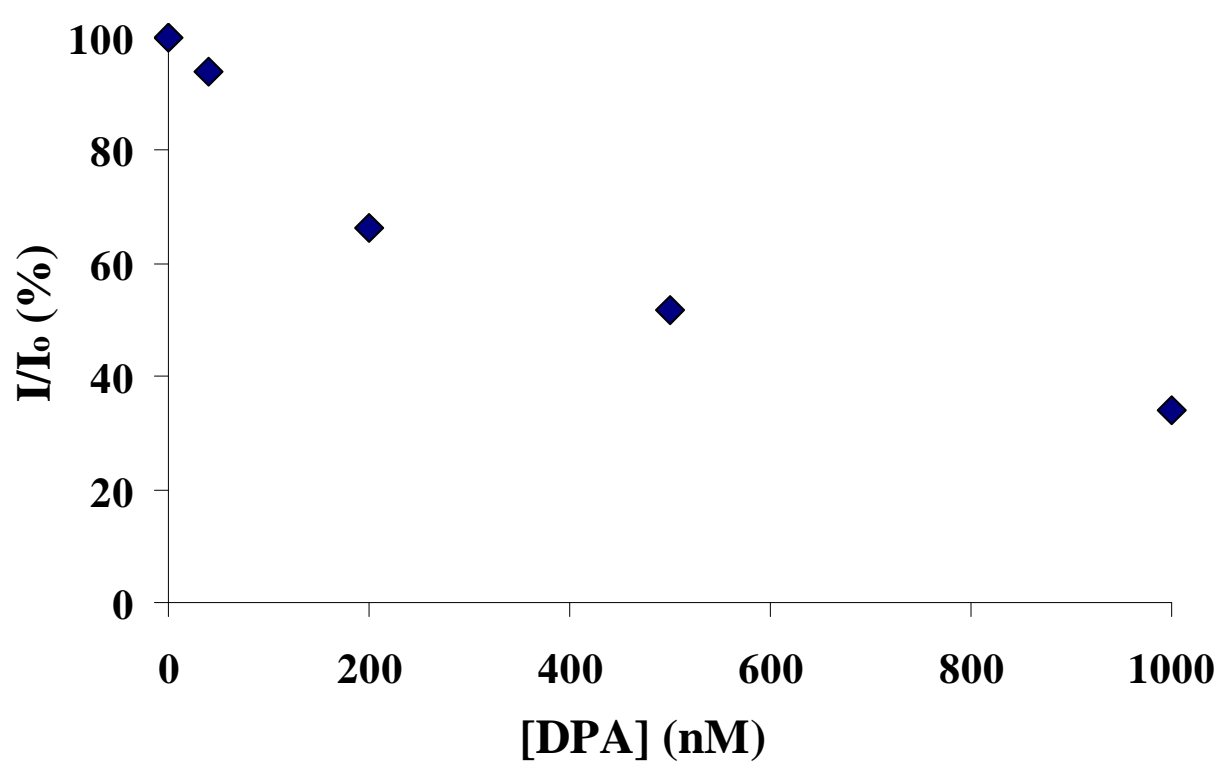

Figure 5.7. The detection sensitivity profile of DPA at $60 \mathrm{~min}$ as a function of different DPA concentrations. 


\subsection{Conclusions}

In conclusion, we have developed a supramolecular sensing platform on a microchip surface that allows the detection of biologically relevant phosphate anions and aromatic carboxylic acids at a high-throughput format. ATP and pyrophosphate among various phosphate anions, and the anthrax biomarker DPA among various aromatic carboxylic acids showed a strong response to the sensitized $\mathrm{Eu}^{3+}$ luminescence-based microchip surface, exhibited by strong quenching of red emission for the anions and DPA in a few to tens of minutes. ATP was sensed by 2-1.Eu ${ }^{3+}$ and showed $\mu \mathrm{M}$ detection sensitivity. A higher detection sensitivity $(\sim 100 \mathrm{nM})$ was achieved for DPA.

Therefore, as a first demonstration of phosphate and carboxylic acid sensing on the same lanthanide-based surface-receptor platform in a microfluidic chip at a high-throughput format, this study offers a general detection platform for phosphates and carboxylic acids in a single test run with high detection sensitivity and selectivity. From this point of view, this supramolecular surface assisted sensing system in multichannel opens new avenues to yield new classes of surface based detection devices for biologically relevant ions and bacterial spores. Real-time imaging and continuous monitoring of analyte solutions with the power of microfluidics in combination with creating functional systems with the power of supramolecular chemistry offers new designs of miniaturized sensing systems for different kinds of small molecules which might have biological and diagnostic importance. Overall, this system exemplifies the significance of microfluidics and noncovalent strategies to create microscale sensing devices by fine-tuning different small building blocks which provide different functionalities when assembled onto such a sensor surface. 


\subsection{Acknowledgement}

The major part of the work presented in this chapter was performed in collaboration with Dr. Bilge Eker in the Mesoscale Chemical Systems group of the University of Twente.

\subsection{Experimental Section}

General Procedures. All reactions involving air- or moisture-sensitive reagents or intermediates were performed under an inert atmosphere of argon in glassware. Unless otherwise noted, solvents and reagents were reagent grade and used without further purification. $\beta$-Cyclodextrin $(\beta C D)$ heptamine, the Eu(III)-EDTA complex 1.Eu ${ }^{3+}$ and the antenna naphthalene $\beta$-diketone 2 were synthesized as described before. ${ }^{[31]}$

Microchip Fabrication Procedure. The silicon-glass microchip was fabricated by following standard microfabrication processes. ${ }^{[32]}$ Microchannels were defined in a (100) silicon wafer (resistivity 5-10 $\Omega \mathrm{cm}$, diameter $100 \mathrm{~mm}$ ) via standard UV-lithography using a mask of photoresist (Olin 907-17 photoresist) followed by deep reactive ion etching (BOSCH-type process). Inlet and outlet holes were fabricated by powderblasting. ${ }^{[33]}$ The silicon wafer was bonded to Borofloat glass via anodic bonding $\left(\mathrm{T}=400{ }^{\circ} \mathrm{C}, \mathrm{U}_{\max }=1200 \mathrm{~V}, \mathrm{t}=10 \mathrm{~min}\right)$ and the silicon glass wafer was diced into separate chips (chips size: $15 \mathrm{~mm}$ x $20 \mathrm{~mm}$ ). Channel dimensions were $50 \mu \mathrm{m} \times 50 \mu \mathrm{m}$ (width $\mathrm{x}$ depth). The length of a single channel of interest was $6 \mathrm{~mm}$ and separation between the channels was $50 \mu \mathrm{m}$.

Surface Modification of the Microchips. The microchannel surface was functionalized with $\beta C D$ SAMs by following a three-step reaction, as described by Ludden et al. ${ }^{[34]}$ Prior to surface modification, intense piranha cleaning was performed inside the microchip via vacuum and then followed by water cleaning. After drying the chip in a stream of $\mathrm{N}_{2}$, 
approximately $500 \mu \mathrm{l}$ freshly distilled toluene was flushed through the chip. Thereafter, a 5 $\mathrm{mM}$ solution of $\mathrm{N}$-[3-(trimethoxysilyl)propyl]ethylenediamine (TPEDA) in toluene was injected through the microchip by the syringe pump with a $0.1 \mu \mathrm{l} / \mathrm{min}$ flow rate for $4 \mathrm{~h}$ at room temperature. Distilled toluene was flushed through the chip for $30 \mathrm{~min}$ at a flow rate of $0.1 \mu \mathrm{l} / \mathrm{min}$ and then followed by flushing at $10 \mu \mathrm{l} / \mathrm{min}$ for $5 \mathrm{~min}$ to get rid of any possible aggregates from the channels. After this step, $10 \mathrm{mM} \mathrm{1,4-phenylene-diisothiocyanate} \mathrm{(DITC)}$ in toluene was flushed inside the microchip and incubated for $4 \mathrm{~h}$ at $60{ }^{\circ} \mathrm{C}$ followed by a rinse with toluene and then with ethanol. After drying the chip, the chip was washed with Millipore water for $5 \mathrm{~min}$, and $10 \mathrm{mM} \beta \mathrm{CD}$ heptamine in Millipore water was injected and incubated in the chip for $2 \mathrm{~h}$ at $60{ }^{\circ} \mathrm{C}$, followed by a rinse of the chip with Millipore water for $5 \mathrm{~min}$.

Assembly of the Sensing Layer on the Microchip Surface. The Eu(III)-EDTA complex was assembled onto the $\beta C D$ monolayers by incubation of the complex in the microchip for $30 \mathrm{~min}$. This results in immobilization of the $\mathrm{Eu}(\mathrm{III})$-EDTA complex onto the $\beta C D$ monolayers via orthogonal supramolecular host-guest interactions. Thereafter, naphthalene $\beta$ diketone as an antenna in water was injected and incubated for $30 \mathrm{~min}$ in the microchip. The self-assembly of the $\mathrm{Eu}(\mathrm{III})$-EDTA conjugate and naphthalene $\beta$-diketone resulted in the formation of a highly red luminescent lanthanide system on the microchannel surface via the energy transfer from the antenna to the surface-anchored $\mathrm{Eu}(\mathrm{III})$.

Microfluidic Fluorescent Sensing Experiments. Different analytes (phosphates such as ATP, ADP, AMP, pyrophosphate, hydrogen phosphate and carboxylic acids such as $o / m / p$ phthalic acids, picolinic acid and dipicolinic acid) in $50 \mathrm{mM}$ HEPES buffer with $50 \mathrm{mM}$ $\mathrm{NaCl}(\mathrm{pH}$ 7.4) were injected from five inlets with a $1 \mu \mathrm{l} / \mathrm{min}$ flow rate at room temperature at a high-throughput format for turn-off sensing experiments. For high-throughput sensing 
experiments, all phosphates and carboxylic acids were used at $1 \mathrm{mM}$ concentration. For detection sensitivity experiments, an ATP concentration down to $50 \mu \mathrm{M}$ and a DPA concentration down to $40 \mathrm{nM}$ was used. The decrease in red emission for each channel in the microchip upon sensing was quantified using a fluorescence microscope using filter $\mathrm{R}$ $\left(\lambda_{\mathrm{em}}=615 \mathrm{~nm}\right)$, and a narrow band excitation filter $\left(300<\lambda_{\mathrm{exc}}<400\right)$ was used to eliminate direct excitation of analytes such as DPA $\left(\lambda_{\mathrm{exc}}=270 \mathrm{~nm}\right)$.

\subsection{References}

[1] a) A. L. Jenkins, O. M. Uy, G. M. Murray, Anal. Chem. 1999, 71, 373-378; b) P. R. Puopolo, P. Chamberlin, J. G. Flood, Clin. Chem. 1992, 38, 1838-1842; c) W. H. van der Schalie, T. R. Shedd, M. W. Widder, L. M. Brennan, J. Appl. Toxicol. 2004, 24, 387-394.

[2] a) M. F. Rega, J. C. Reed, M. Pellecchia, Bioorg. Chem. 2007, 35, 113-120; b) P. J. Hajduk, T. Gerfin, J. M. Boehlen, M. Haberli, D. Marek, S. W. Fesik, J. Med. Chem. 1999, 42, 2315-2317; c) M. A. Huestis, M. L. Smith, Drug Discov. Today 2006, 3, 49-57.

[3] a) E. Fu, T. Chinowsky, K. Nelson, K. Johnston, T. Edwards, K. Helton, M. Grow, J. W. Miller, P. Yager, Ann. NY. Acad. Sci. 2007, 1098, 335-344; b) A. M. Smith, S. Dave, S. Nie, L. True, X. Gao, Expert Rev. Mol. Diagn. 2006, 6, 232-244; c) P. Haas, P. Then, A. Wild, W. Grange, S. Zorman, M. Hegner, M. Calame, U. Aebi, J. Flammer, B. Hecht, Anal. Chem. 2010, 82, 6299-6302.

[4] a) E. Lester, A. Ponce, IEEE Eng. Med. Biol. 2002, 38-42; b) E. Lester, A. Ponce, IEEE Eng. Med. Biol. 2004, 130-135. 
[5] a) J. R. Lakowicz, in Principles of Fluorescence Spectroscopy, Kluwer Academic and Plenum Publishers, New York, $2^{\text {nd }}$ edn. 1999; b) R. Narayanaswamy and O. S. Wolfbeis (eds.), in Optical Sensors: Industrial, Environmental and Diagnostic Applications, Springer Series on Chemical Sensors and Biosensors, Berlin, 2004, vol. 1; c) R. Martinez - Manez, F. Sancenon, J. Fluoresc. 2005, 15, 267-285; d) R. Martinez - Manez, F. Sancenon, Chem. Rev. 2003, 103, 4419-4476.

[6] a) K. Niikura, A. Metzger, E.V. Anslyn, J. Am. Chem. Soc. 1998, 120, 8533-8534; b) A. Metzger, E.V. Anslyn, Angew. Chem. Int. Ed. 1998, 37, 649-652.

[7] a) N. S. Murray, S. P. Jarvis, T. Gunnlaugsson, Chem. Commun. 2009, 33, 49594961; b) J. Massue, S. J. Quinn, T. Gunnlaugsson, J. Am. Chem. Soc. 2008, 130, 6900-6901.

[8] a) M. L. Cable, J. P. Kirby, K. Sorasaenee, H. B. Gray, A. Ponce, J. Am. Chem. Soc. 2007, 129, 1474-1475; b) K. Ai, B. Zhang, L. Lu, Angew. Chem. Int. Ed. 2009, 48, 304-308; c) M. D. Yilmaz, S. Hsu, D. N. Reinhoudt, A. H. Velders, J. Huskens, Angew. Chem. Int. Ed. 2010, 49, 5938-5941.

[9] a) J. P. Leonard, C. B. Nolan, F. Stomeo, T. Gunnlaugsson, Top Curr. Chem. 2007, 281, 1; b) J. P. Leonard, P. Jensen, T. McCabe, J. E. O’Brien, R. D. Peacock, P. E. Kruger, T. Gunnlaugsson, J. Am. Chem. Soc. 2007, 129, 10986; c) T. Gunnlaugsson, F. Stomeo, Org. Biomol. Chem. 2007, 5, 1999.

[10] K. Binnemans, Chem. Rev. 2009, 109, 4283-4374.

[11] a) R. M. Crooks, A. J. Ricco, Acc. Chem. Res. 1998, 31, 219-227; b) A. E. Kaifer, Isr. J. Chem. 1996, 36, 389-397.

[12] a) R. Zimmerman, L. Basabe-Desmonts, F. van der Baan, D. N. Reinhoudt, M. CregoCalama, J. Mater. Chem. 2005, 15, 2772-2777; b) Y. R. Kim, J. J. Kim, J. S. Kim, H. Kim, Adv. Mater. 2008, 20, 4428-4432. 
[13] a) S. Flink, F. C. J. M. van Veggel and D. N. Reinhoudt, Adv. Mater. 2000, 12, 13151328; b) N. J. van der Veen, S. Flink, M. A. Deij, R. J. M. Egberink, F. C. J. M. van Veggel, D. N. Reinhoudt, J. Am. Chem. Soc. 2000, 122, 6112-6113; c) M. CregoCalama, D. N. Reinhoudt, Adv. Mater. 2001, 13, 1171-1174.

[14] a) L. Basabe-Desmonts, J. Beld, R. S. Zimmerman, J. Hernando, P. Mela, M. F. Garcia Parajo, N. F. Van Hulst, A. van den Berg, D. N. Reinhoudt, M. Crego-Calama, J. Am. Chem. Soc. 2004, 126, 7293-7299; b) C. M. Rudzinski, A. M. Young, D. G. Nocera, J. Am. Chem. Soc. 2002, 124, 1723-1727.

[15] J. M. Berg, J. L. Tymoczko, L. Stryer, in Biochemistry, W.H. Freeman, New York, $5^{\text {th }}$ edn. 2002.

[16] A. Ojida, I. Takashima, T. Kohira, H. Nonaka, I. Hamachi, J. Am. Chem. Soc. 2008, 130, 12095-12101.

[17] P.D. Beer, P.A. Gale, Angew. Chem. Int. Ed. 2001, 40, 486.

[18] Q. Li, P. K. Dasgupta, H. Temkin, Environ. Sci. Technol. 2008, 42, 2799-2804.

[19] a) D. A Henderson, Science 1999, 283, 1279-1282; b) M. Enserink, Science 2001, 294, 1266-1267; c) P. T. Yung, E. D. Lester, G. Bearman, A. Ponce, Biotechnol. Bioeng. 2007, 98, 864-871.

[20] a) G. F. Bailey, S. Karp, L. E. Sacks, J. Bacteriol. 1965, 89, 984; b) D. R. Walt, Anal. Chem. 2000, 72, 738a- 746a; c) L. J. Rode, J. W. Foster, Nature 1960, 188, 11321134.

[21] D. L. Rosen, Rev Anal. Chem. 1999, 18, 1-21.

[22] a) P. Mela, S. Onclin, M. H. Goedbloed, S. Levi, M. F. Garcia-Parajo, N. F. van Hulst, B. J. Ravoo, D. N. Reinhoudt, A. van den Berg, Lab Chip 2005, 5, 163-170; b) T. Vilkner, D. Janasek, A. Manz, Anal. Chem. 2004, 7612, 3373-3385; c) H. Andersson, A. Van den Berg, Sens. Actuators B. 2003, 92, 315-325; d) H. Y. Fan, F. 
Y. Lu, A. Stump, S. T. Reed, T. Baer, R. Schunk, V. Perez - Luna, G. P. Lopez, C. J. Brinker, Nature 2000, 405, 56 - 60 .

[23] A. Mulder, J. Huskens, D. N. Reinhoudt, Org. Biomol. Chem. 2004, 2, 3409.

[24] M. J. W. Ludden, D. N. Reinhoudt, J. Huskens, Chem. Soc. Rev. 2006, 35, 11221134.

[25] a) A. Metzger, V. M. Lynch, E. V. Anslyn, Angew. Chem. Int. Ed. 1997, 36, 862-865.

b) M. J. Berridge, Nature 1993, 361, 315 .

[26] N. Shao, J. Jin, G. Wang, Y. Zhang, R. Yang, J. Yuan, Chem. Commun. 2008, 11271129

[27] a) L. J. Charbonniere, R. Schurhammer, S. Mameri, G. Wipff, R. F. Ziessel, Inorg. Chem. 2005, 44, 7151-7160; b) S. M. Shanbhag, G. R. Choppin, Inorg. Chim. Acta 1987, 139, 119-120.

[28] a) Y. Kanekiyo, R. Naganawa, H. Tao, Chem. Commun. 2004, 1006-1007; b) C. Bazzicalupi, S. Biagini, A. Bencini, E. Faggi, C. Giorgi, I. Matera, B. Valtancoli, Chem. Commun. 2006, 4087-4089.

[29] G. V. Zyryanov, M. A. Palacios, P. Anzenbacher, Jr., Angew. Chem. Int. Ed. 2007, 46, 7849-7852.

[30] a) J. P. Kirby, M. L. Cable, D. J. Levine, H. B.Gray, A. Ponce, Anal Chem. 2008, 80, 5750-5754; b) S. L. Wu, W. D. Horrocks, Jr., Anal. Chem. 1996, 68, 394-401.

[31] a) P. R. Ashton, R. Königer, and J. F. Stoddart, D. Alker ,V. D. Harding, J. Org. Chem. 1996, 61, 903-908; b) S. H. Hsu, M. D. Yilmaz, C. Blum, V. Subramaniam, D. N. Reinhoudt, A. H. Velders, J. Huskens, J. Am. Chem. Soc. 2009, 131, 12567-12569; c) J. Yuan, K. Matsumoto, Anal. Sci. 1996, 12, 31- 36.

[32] J. G. E Gardeniers, R. E. Oosterbroek, A. van den Berg, in: Lab-on-a-chip: Miniaturized systems for (bio)chemical analysis and synthesis, Elsevier, Amsterdam, 
2003, 37-64.

[33] H. Wensink, M. C. Elwenspoek, Wear 2002, 253, 1035-1043.

[34] M. J. W. Ludden, X. Y. Ling, T. Gang, W. P. Bula, H. J. G. E. Gardeniers, D. N.

Reinhoudt, J. Huskens, Chem. Eur. J. 2008, 14, 136-142. 


\section{Chapter 6}

\section{Local Doping of Silicon Using Nanoimprint Lithography and Molecular Monolayers*}

Two fabrication schemes for the direct patterning of organic monolayers on oxide-free silicon are reported combining top-down nanoimprint lithography and bottom-up monolayer formation. The first approach was designed to form monolayer patterns on the imprinted areas, while the second approach was designed for monolayer formation outside of the imprinted features. By both approaches, covalently bonded Si-C monolayer patterns with feature sizes ranging from $100 \mathrm{~nm}$ to $100 \mu \mathrm{m}$ were created via a hydrosilylation procedure using diluted reagents. This novel patterning strategy was successfully applied for introducing dopant atoms using a phosphorus-containing molecular precursor on oxide-free silicon. The patterned sample was protected by a $\mathrm{SiO}_{2}$ capping layer applied by e-beam evaporation and subjected to rapid thermal annealing (RTA) to diffuse the phosphorus dopant atoms into the bulk silicon locally. The doped sample was investigated by $3 D$ time-offlight secondary ion mass spectrometry (TOF-SIMS) and electrically characterized by Hall and Van der Pauw measurements.

* Parts of this chapter have been published in: W. Pim Voorthuijzen, M. Deniz Yilmaz, Alberto Gomez-Casado, Pascal Jonkheijm, Wilfred G. van der Wiel, Jurriaan Huskens, Langmuir, 2010, 26, 14210-14215; W. Pim Voorthuijzen, M. Deniz Yilmaz, Wouter J. B. Naber, Jurriaan Huskens, Wilfred G. van der Wiel, Adv. Mater. 2011, in press. 


\subsection{Introduction}

A vast amount of semiconductor technology is focused on downscaling electronic components according to Moore's Law. ${ }^{[1]}$ Silicon's semiconducting properties largely depend on the concentration of dopant impurities. ${ }^{[2]}$ Therefore, novel methodologies of selectively introducing dopants to semiconductor materials at the nanometer scale could be beneficiary for the development of smaller IC (integrated circuit) components.

Conventionally, introducing dopant impurities to silicon is achieved using ion implantation. ${ }^{[3]}$ In this process, an ion beam is used to bombard the surface by highly energetic dopant ions, literally shooting the dopants in the top layers of the bulk silicon. True electrical activation is then achieved using a high-temperature annealing step. This fabrication step is required for repairing the - by bombardment inflicted - crystal damage followed by diffusion of (near) surface located dopant atoms.

Fabrication of ultra-shallow, heavily doped junctions including well-defined insulating areas will be required for reliably manufacturing smaller scale devices. ${ }^{[1,4,5]}$ In this respect, the ability for a true deterministic positioning i.e. control over the exact position of impurity atoms in semiconductor structures will become essential. A possible methodology would involve control over lateral $(x-y)$ positioning, concomitant with considerate influence on the penetration depth of dopant diffusion in the $z$ direction from the surface.

In 2008, the group of Javey published a novel method for introducing dopants using molecular monolayers on oxide-free silicon. ${ }^{[6]}$ Using this method, the authors showed it was possible to obtain high-level doping of silicon by application of hydrosilylation surface chemistry on $\mathrm{Si}-\mathrm{H}$ terminated silicon ${ }^{[7,8]}$ with dopant atom-containing organic monolayers. Rapid thermal annealing (RTA) ensures implantation of dopant atoms by diffusion. Using RTA, this method obviates the relatively long annealing steps required to repair damage to the silicon crystal structure. Therefore, a first requirement in deterministic positioning of 
dopant atoms is met, in the sense that, using RTA, dopant atoms mainly end up in the top 100 $\mathrm{nm}$ of the silicon substrate. Another benefit of the aforementioned strategy is the use of a limited source, such as a molecular monolayer, which allows specific tuning of the dopant surface dose ${ }^{[6]}$ Moreover, molecular (self-) assembly is a cheap bottom-up nanofabrication method. ${ }^{[9]}$

Introducing dopants by molecular monolayers therefore in principle offers considerable advantages. However, actual device fabrication necessitates combination of the approach of Javey et al. with the ability to selectively pattern and therefore control over the lateral (x-y) positioning of molecular monolayer structures.

Although full, covalently bonded Si-C monolayers are relatively well developed and hold great promise for novel applications, procedures for the nano- or micro structuring of the oxide free silicon surface remain limited to a few examples. The first report by Effenberger et al. ${ }^{[10]}$ described the generation of patterns on the hydrogen-terminated silicon surface by use of a conventional photomask and UV light. Both neat aldehydes and diluted alkenes were used for monolayer formation, resulting in covalent $\mathrm{Si}-\mathrm{O}-\mathrm{C}$ and $\mathrm{Si}-\mathrm{C}$ bonds, respectively. In a similar approach by Wojtyk et al., ${ }^{[11]}$ fully hydrogen-terminated samples were partially oxidized in UV light, while pressing a gold raster against the hydrogen-passivated $\mathrm{Si}(111)$ surface to shield part of the substrate from the light. After oxidation of exposed features, the remaining hydrogen-terminated areas were further functionalized with a terminal alkene. Cathodic electrografting by STM using 1-alkynes was demonstrated by Hurley et al. ${ }^{[12]}$ Sub$100 \mathrm{~nm}$ patterning by use of catalytic stamp lithography was achieved by Mizuno et al. ${ }^{[13]}$ using palladium nanoparticles. The feature sizes and lateral positioning attainable with this method are directed by nucleation and growth of the nanoparticles, and sub- $20 \mathrm{~nm}$ patterning was demonstrated. Recently, sub- $80 \mathrm{~nm}$ patterns of $\mathrm{SiO}_{2}$ were obtained by Klingebiel et al. by locally decomposing alkyl monolayers on silicon using photothermal laser processing. ${ }^{[14]}$ 
These existing procedures suffer from one or more limitations regarding (i) the use of certain reagents (for example, contamination by metals, potentially used as catalysts, needs to be avoided for microelectronic applications), (ii) the ability to fabricate well packed layers, (iii) the patterning of nanometer-sized features, (iv) the control over feature size and position by using arbitrary master structures, and (v) throughput or possibilities for assembly on full wafer scale. Here, we show that monolayer patterns from $100 \mathrm{~nm}$ to $100 \mu \mathrm{m}$ can be successfully created using a combination of top-down and bottom-up fabrication methods: imprint lithography and molecular monolayer formation, respectively. We use thermal nanoimprint lithography (NIL), ${ }^{[15]}$ a hot embossing technique that is suitable for nanofabrication down to sub-10 nm structures ${ }^{[16]}$ to generate patterns of molecular monolayers on oxide-free silicon. NIL is applicable in both regular lab and cleanroom conditions, allows relatively high throughput, and is applicable on full wafer scale. A key characteristic of the thermal NIL process is the use of a thermoplastic polymer as a resist material. In the targeted combined process with monolayer formation on $\mathrm{Si}$, such a resist needs to be resistant against wet fluoride etching, which is used to remove the native oxide, in order that a regular, catalyst-free hydrosilylation procedure can be used for the subsequent monolayer attachment. Two different fabrication schemes are reported by which, using the same imprint mold, monolayer patterns can be obtained, as well as the inverse of these patterns. This novel patterning strategy can be furthermore used for the doping of nearly intrinsic silicon using a phosphorus-containing organic precursor.

\subsection{Results and discussion}

\subsubsection{NIL-patterned monolayers on silicon}

Scheme 6.1 outlines two possible approaches toward the structuring of organic monolayers on oxide-free silicon. The first approach (Scheme 6.1A, direct method) typically starts from a clean $\mathrm{Si} / \mathrm{SiO}_{2}$ sample. In step a, the desired polymeric resist material is applied 
to the sample by spin coating. In steps $b(\mathrm{NIL})$ and $\mathrm{c}\left(\mathrm{O}_{2}\right.$ plasma etching), the pattern on the mold is transferred into the resist layer. In step d, the exposed $\mathrm{SiO}_{2}$ areas are subjected to wet etching in aqueous $\mathrm{NH}_{4} \mathrm{~F}$, which effectively generates a H-terminated $\mathrm{Si}$ surface. The remaining resist is removed (step e), and the monolayer is formed on the $\mathrm{Si}-\mathrm{H}$ surface areas.

(A)

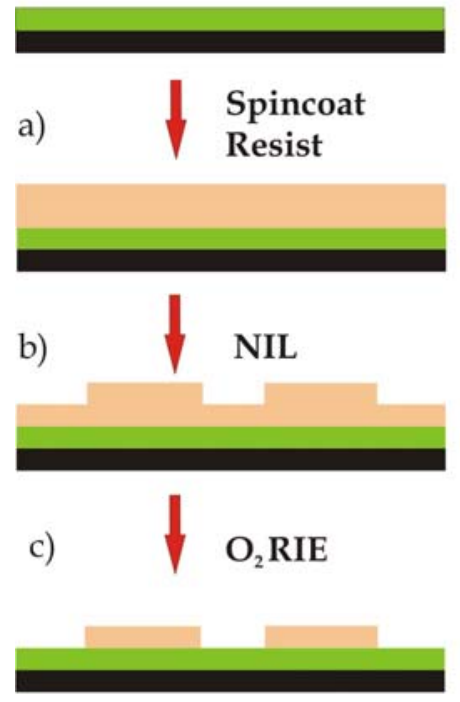

d)
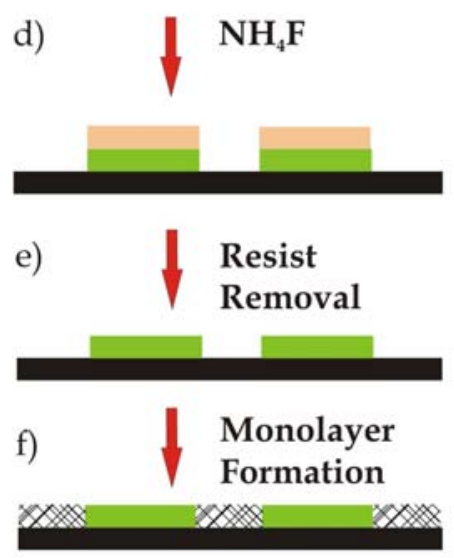

Silicon Native $\mathrm{SiO}_{2}$
(B)

a) $\quad \mathrm{NH}_{4} \mathrm{~F}$

b) $\quad \begin{aligned} & \text { Monolayer } \\ & \text { Formation }\end{aligned}$

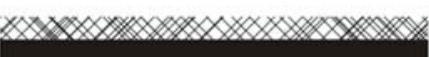

c) $\quad \begin{aligned} & \text { Spincoat } \\ & \text { Resist }\end{aligned}$

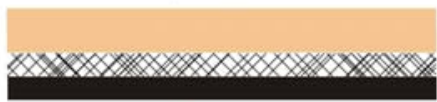

d) NIL

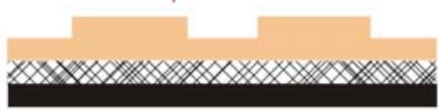

e) $\quad \mathrm{O}_{2} \mathrm{RIE}$
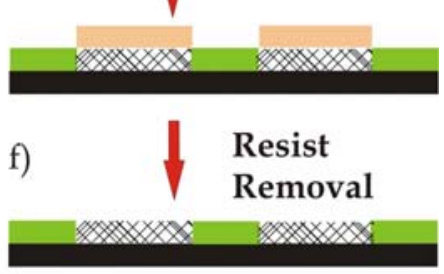

Resist

Si-C bonded Monolayer

Scheme 6.1 Fabrication routes to the direct (A) and inverse (B) patterning of monolayers on the $\mathrm{Si}-\mathrm{H}$ surface. 
In the second approach (Scheme 6.1B, inverse method), the native oxide layer is completely stripped from the silicon sample by $\mathrm{NH}_{4} \mathrm{~F}$ (step a), which leaves a H-terminated surface, and a full monolayer is formed on the substrate (step b). The polymeric resist is applied on top of the monolayer by spin coating (step c). After imprinting (step d), $\mathrm{O}_{2}$ plasma etching (step e) ensures the removal of both the residual resist layer and the monolayer underneath, and allows for reoxidation of the exposed $\mathrm{Si}$ surface areas. Hence, after completion of the pattern transfer procedure by resist removal (step f), the inverse pattern is obtained.

The procedure reported by Sieval et al. ${ }^{[17]}$ was used for all monolayer formation steps by the thermal method using mesitylene as the solvent. Initially, 1-hexadecene was employed as the molecular precursor, since it is known to give stable, well-packed monolayers with a high degree of order and crystallinity. ${ }^{[17,18,19]}$ Full monolayers showed, as expected, high advancing water contact angles $\left(\theta_{\mathrm{A}}>111^{\circ}\right)$, and their formation was further supported by X-ray photoelectron spectroscopy (XPS; data not shown).

Monolayer patterns with $100-\mu \mathrm{m}$ features were fabricated using the procedure outlined in Scheme 1A (direct method). The feature size was selected to enable pattern characterization by XPS. A layer of resist $(10 \mathrm{wt} \%$ solution of $350 \mathrm{kD}$ poly(methyl methacrylate) (PMMA) in toluene) was applied by spin coating onto a freshly cleaned Si(111) sample (with the native oxide) and soft-baked in an oven $\left(60^{\circ} \mathrm{C}, 5 \mathrm{~min}\right)$, resulting in an ellipsometric thickness of around $1080 \mathrm{~nm}$. The sample was then imprinted (40 bar, 180 ${ }^{\circ} \mathrm{C}, 30 \mathrm{~min}$ ) using a mold with $100 \mu \mathrm{m}$ diameter pillars separated by $125 \mu \mathrm{m}$ and with a feature height of $450 \mathrm{~nm}$. Anisotropic reactive ion etching (RIE) with $\mathrm{O}_{2}$ plasma was performed to remove the residual layer. In order to remove $\mathrm{SiO}_{2}$ from the exposed surface areas, the sample was subjected for $15 \mathrm{~min}$ to a deoxygenated, argon-sparged, $40 \mathrm{wt} \% \mathrm{NH}_{4} \mathrm{~F}$ solution, a procedure known to result in removal of $\mathrm{SiO}_{2}$ and to yield an atomically flat 
hydrogen-terminated Si (111) surface. ${ }^{[26]}$ After rinsing with water and drying in a stream of $\mathrm{N}_{2}$, the remaining PMMA was removed in acetone. The sample was rinsed with deoxygenated water, dried in a stream of $\mathrm{N}_{2}$, and immediately inserted into a reaction flask together with $1 \mathrm{~mL}$ of a deoxygenated solution of $25 \% \mathrm{v} / \mathrm{v} 1$-hexadecene in mesitylene. The monolayer was formed under reflux. After cooling and rinsing, physisorbed material was removed by ultrasonication in acetone for $15 \mathrm{~min}$.

To visualize the patterns by XPS, scanning X-ray imaging (SXI) was performed, which provides a secondary electron image with darker and brighter areas corresponding to differences in the amounts of detected backscattered secondary electrons. Also, elemental mapping spectra were generated by scanning over the sample. Figure $6.1 \mathrm{a}$, b shows $\mathrm{O}_{1 \mathrm{~s}}$ and $\mathrm{Si}_{2 \mathrm{p}}$ spectra, respectively. Mapping of the high binding energy part of the averaged $\mathrm{O}_{1 \mathrm{~s}}$ spectrum resulted in the appearance of a dot pattern (Figure 6.1a) with the expected feature sizes and period. The bright areas indicate a richness in oxygen, corresponding to the presence of $\mathrm{SiO}_{2}$. Mapping of the $\mathrm{Si}_{2 p}$ signal was done using the low binding energy part of the spectrum (Figure 6.1b), which is known to correspond to less oxidized silicon, ${ }^{[20]}$ which thus signals the presence of silicon and $\mathrm{Si}-\mathrm{C}$. Therefore, the bright areas indicate the successful removal of $\mathrm{SiO}_{2}$ in these areas.

In order to study the stability of the monolayer surface patterns against reoxidation, the sample was stored for 1 month under ambient conditions. The sample was reinspected by XPS. The SXI image (Figure 6.1c) shows the excellent feature stability. Elemental information was obtained from line scans (Figure 6.1d). Clear differences in atomic concentrations mark the edges of the dots. Outside of the dots, high signal intensities of $\mathrm{Si}_{2 \mathrm{p}}$ (high energy side, indicating oxygen-rich $\mathrm{Si}$ and thus signaling the presence of $\mathrm{SiO}_{2}$ ) and an $\mathrm{O}_{1 \mathrm{~s}}$ signal were observed, while the $\mathrm{C}_{1 \mathrm{~s}}$ signal was higher in the dot areas. These data confirm that the patterned monolayers are still intact. 
a
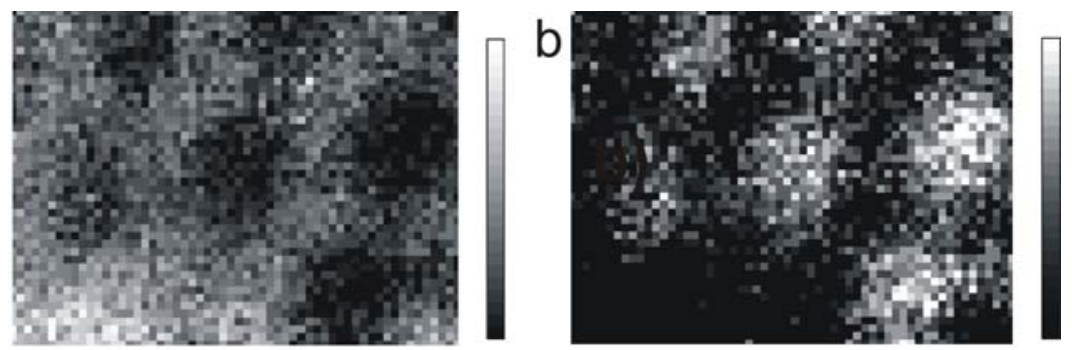

C
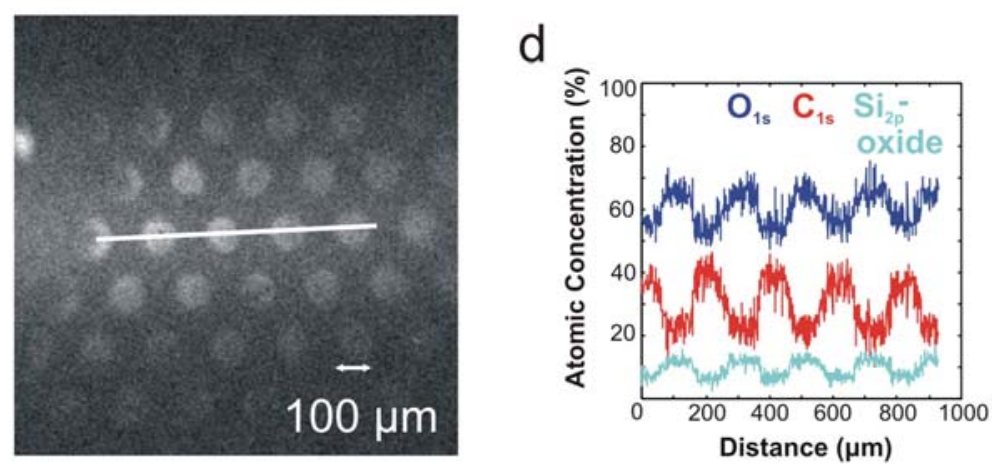

Figure 6.1 XPS elemental mapping of (a) the $\mathrm{O}_{1 \mathrm{~s}}$ signal of the high binding energy side and of (b) the $\mathrm{Si}_{2 \mathrm{p}}$ signal of the low binding energy side. SXI image (c) after 30 days of storage under ambient conditions. The line marks the line scans reported in panel $\mathrm{d}$.

The direct patterning method (Scheme 6.1A) was subsequently used for the replication of nanoscale features of $150 \mathrm{~nm}$ diameter dots. To this purpose, a nickel-plated mold containing a square array of $150 \mathrm{~nm}$ pillars in diameter at a $500 \mathrm{~nm}$ period with a feature height of $100 \mathrm{~nm}$ was imprinted into a 200-nm layer of the commercially available resist mr-I 7020E, which was applied by spin coating followed by a short baking step. Residual layer removal was conducted for $90 \mathrm{~s}$.

In an attempt to visualize the pattern and to accurately measure the height of the hexadecyl monolayer, the sample was subjected to an $\mathrm{NH}_{4} \mathrm{~F}$ etch to remove the residual $\mathrm{SiO}_{2}$ between the dots. Because of the resistance of alkyl-patterned silicon surfaces against fluoride etching, this etching step should leave the monolayer intact. ${ }^{[21,27]}$ The pattern was visualized by contact-mode atomic force microscopy (AFM), and a clearly defined pattern 
was obtained (Figure 6.2a). The extracted height profile (Figure 6.2b), however, showed a measured feature height of $\sim 4 \mathrm{~nm}$, while $1.8 \mathrm{~nm}$ is expected for a hexadecyl monolayer. ${ }^{[19]} \mathrm{A}$ contact-mode AFM height image of a $100 \mu \mathrm{m}$ dot for which the residual $\mathrm{SiO}_{2}$ layer was not removed by fluoride etching indicated a height difference of $\sim 2 \mathrm{~nm}$ (the $\mathrm{SiO}_{2}$ area being the higher one), which would indicate a $\mathrm{SiO}_{2}$ layer with a thickness of $\sim 6 \mathrm{~nm}$. Despite the height difference not yet being fully understood, the pattern quality clearly shows that nanoscale patterning is also possible using this method.
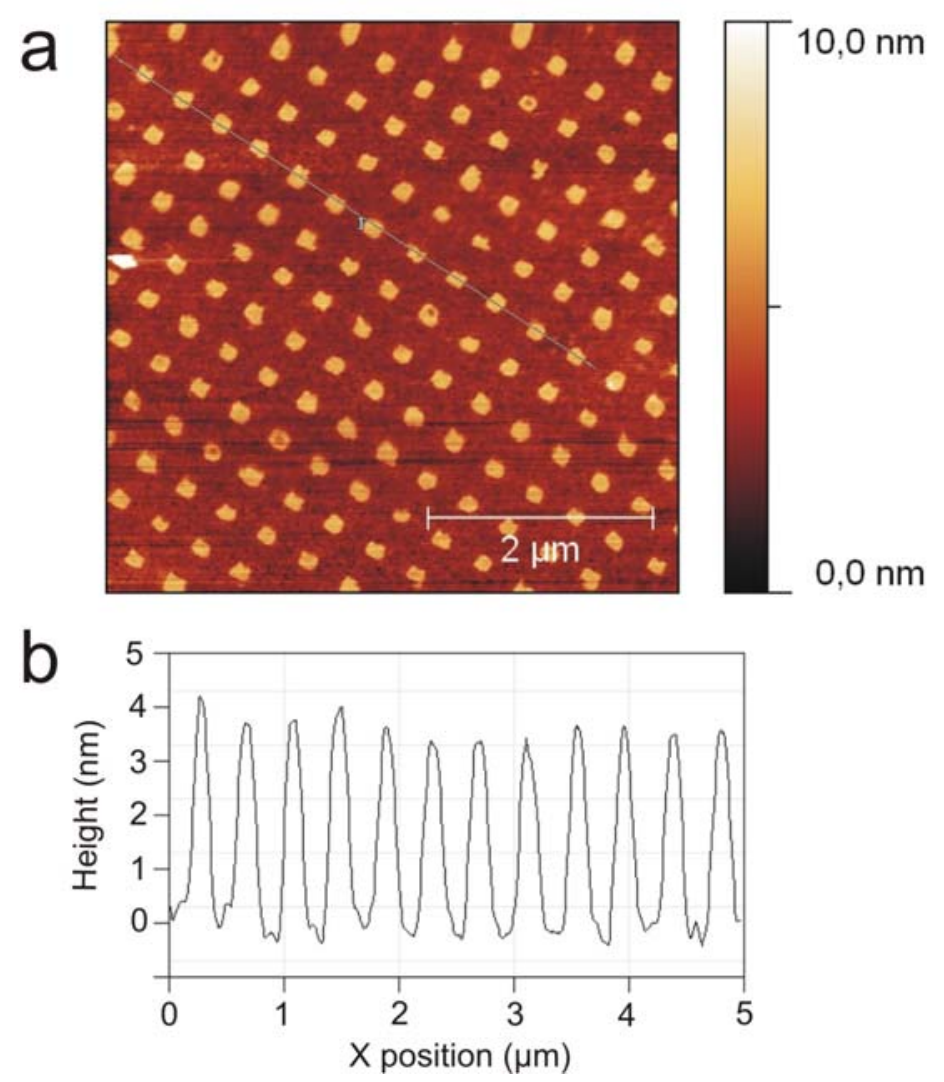

Figure 6.2 Contact-mode AFM height image (a) and corresponding line profile (b) of a 150 $\mathrm{nm}$ diameter dot pattern with a $500 \mathrm{~nm}$ period of a hexadecyl monolayer patterned on oxidefree silicon (Scheme 6.1A). The sample was exposed to $\mathrm{NH}_{4} \mathrm{~F}$ to remove the $\mathrm{SiO}_{2}$ between the monolayer areas. 
Subsequently, hexadecyl monolayer patterns were fabricated according to the inverse procedure outlined in Scheme 6.1B. To this purpose, a clean $\mathrm{Si} / \mathrm{SiO}_{2}$ sample was fully stripped of its native $\mathrm{SiO}_{2}$ in aqueous $\mathrm{NH}_{4} \mathrm{~F}$ (Scheme 6.1B, step a) followed by rinsing with water. Monolayers were formed under thermal conditions as described above (Scheme 6.1B, step b). After rinsing the sample with acetone, ethanol and water, and drying in a stream of $\mathrm{N}_{2}$, water contact angle measurements indicated the formation of a hydrophobic surface $\left(\theta_{\mathrm{a}}=111-113^{\circ}\right)$. A 760-nm layer of PMMA was deposited by spin coating and soft-baking for $5 \mathrm{~min}$ at $60{ }^{\circ} \mathrm{C}$. The sample was imprinted using a mold containing $3-\mu \mathrm{m}$ ridges with an $8-\mu \mathrm{m}$ period with a feature height of $550 \mathrm{~nm}\left(40 \mathrm{bar}, 180{ }^{\circ} \mathrm{C}, 15 \mathrm{~min}\right.$, Scheme $6.1 \mathrm{~B}$, step d). $\mathrm{O}_{2}$ plasma etching ( $3 \mathrm{~nm} / \mathrm{s}$ for $2 \mathrm{~min}$ ) ensured removal of the residual layer and the monolayer parts underneath the imprinted features (Scheme 6.1B, step e). The oxygen plasma applied in this etching step is expected to cause immediate reoxidation of the exposed features. Lift-off by ultrasonication in acetone resulted in the final patterned sample (Scheme 6.1B, step f).

Before contact mode AFM imaging, we removed the $\mathrm{SiO}_{2}$ on the parts not covered with a monolayer by aqueous $\mathrm{NH}_{4} \mathrm{~F}$ etching. The resulting height image is depicted in Figure 6.3a. From the corresponding height profile (Figure 6.3b), we obtained a pattern height of 1.7 $\mathrm{nm}$. This height agrees excellently with the expected $1.8 \mathrm{~nm} .^{[19]}$

The same procedure (Scheme 6.1B) was applied to nanometer-scale features. To this purpose, hexadecyl monolayers were prepared in a similar manner, now using a 130-nm resist layer and a master with 100 -nm ridges separated by alternating spacings of 3 and 5 $\mu \mathrm{m} .{ }^{[22]} \mathrm{O}_{2}$ plasma etching and resist removal gave the resulting pattern. Before AFM imaging, the exposed $\mathrm{SiO}_{2}$ was removed. Figure 6.3c shows the resulting image, and the corresponding line profile is found in Figure 6.3d. The line width (approximately $200 \mathrm{~nm}$ ) has increased somewhat compared to the original master, which is attributed to the roundness of the ridges of this particular master, leading to pattern widening upon prolonged etching. ${ }^{[22]}$ 
a

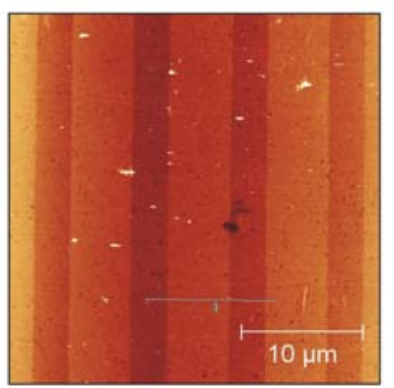

C

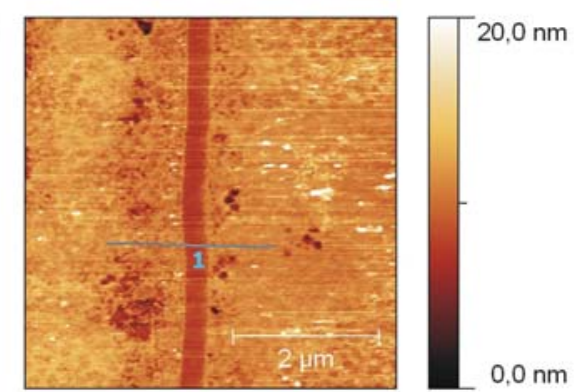

b

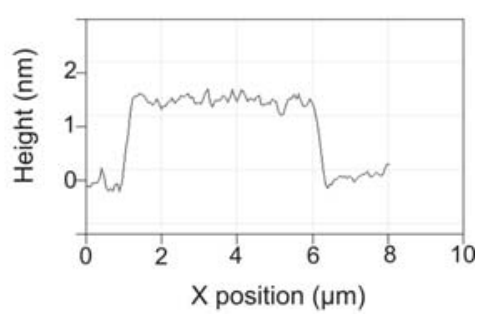

d

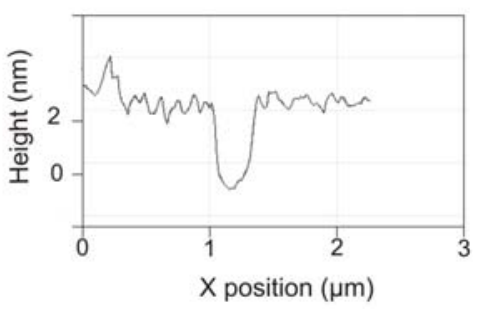

Figure 6.3 Contact-mode AFM height images (a,c) and corresponding height profiles $(b, d)$ of micro- $(\mathrm{a}, \mathrm{b})$ and nano- $(\mathrm{c}, \mathrm{d})$ patterned hexadecyl monolayers, created using the inverse method (Scheme 6.1B). The samples were exposed to aqueous $\mathrm{NH}_{4} \mathrm{~F}$ before imaging.

The observed height $(2 \mathrm{~nm})$ again agrees well with the height of the hexadecyl monolayer. These results show that the inverse method can also be applied to create high-resolution monolayer features on Si. When looking at the heights of the features, the inverse method (Scheme 6.1B) demonstrates a higher degree of reproducibility compared to the direct method (Scheme 6.1A).

To demonstrate the applicability of functional molecules, patterns of a fluorescent dye were prepared. Because of quenching of fluorescence close to the silicon surface, ${ }^{[23]}$ we chose a fluorophore based on a boron-dipyrrin (BODIPY) ${ }^{[24]}$ structure known for its high quantum yield, ${ }^{[25]}$ and the alkene-terminated derivative 1 (Figure 6.4a) was synthesized. A full monolayer of $\mathbf{1}$ was formed in refluxing mesitylene overnight. After monolayer formation, the sample was rinsed with acetone, ethanol, and water. After drying in a stream of $\mathrm{N}_{2}$, water contact angle measurements $\left(\theta_{\mathrm{a}}=91^{\circ}\right)$ indicated the formation of a more 
hydrophobic surface compared to a freshly hydrogen-terminated silicon surface $\left(\theta_{\mathrm{a}} \sim 78^{\circ}\right)$. Line patterns of $100 \mu \mathrm{m}$ width at $200 \mu \mathrm{m}$ period were fabricated subsequently, according to the inverse method (Scheme 6.1B).

The resulting pattern was imaged by fluorescence microscopy (Figure 6.4a). The fluorophore is excited at $450-480 \mathrm{~nm}$ and emits in the visible green $\left(\lambda_{\mathrm{ex}} \geq 515 \mathrm{~nm}\right)$. A clear pattern definition is observed. The spacer length ( 11 carbon atoms, about $1.5 \mathrm{~nm})$ used here is apparently sufficient to observe the pattern. A longer alkyl spacer will likely further reduce quenching by the substrate. ${ }^{[23]}$ These initial results confirm that functional molecules can also be patterned using the methods developed in this study.

a

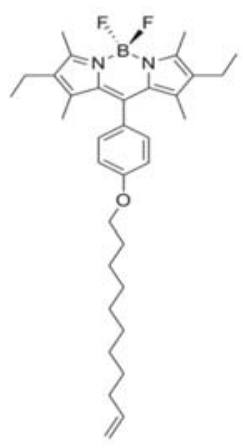

b

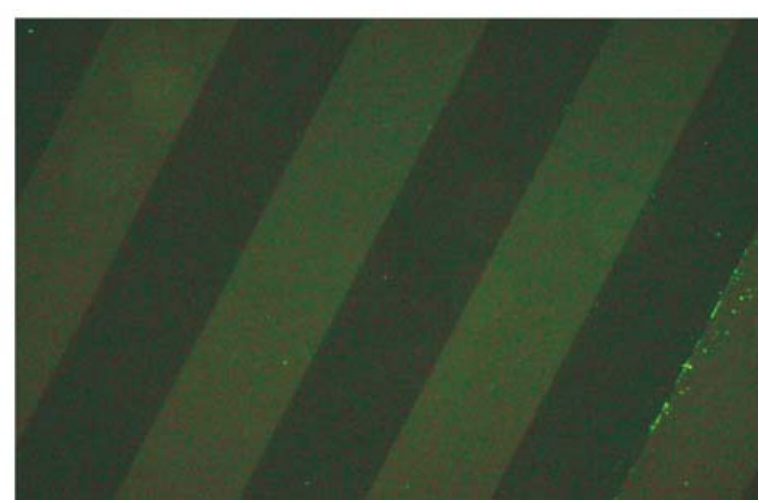

Figure 6.4 Alkene-terminated BODIPY derivative 1 (a) and fluorescence microscopy image (b) of 100- $\mu \mathrm{m}$ wide lines of $\mathbf{1}$ fabricated using the inverse method (Scheme 6.1B).

\subsubsection{Local doping of silicon by NIL-patterning, monolayer formation and rapid thermal annealing}

Using a phosphorus-containing organic precursor, highly-doped and patterned ( $\mu \mathrm{m}-$ scale) regions in nearly intrinsic silicon were successfully fabricated. These patterned regions were characterized by time-of-flight secondary ion mass spectrometry (TOF-SIMS), and by Hall and sheet-resistance measurements. 


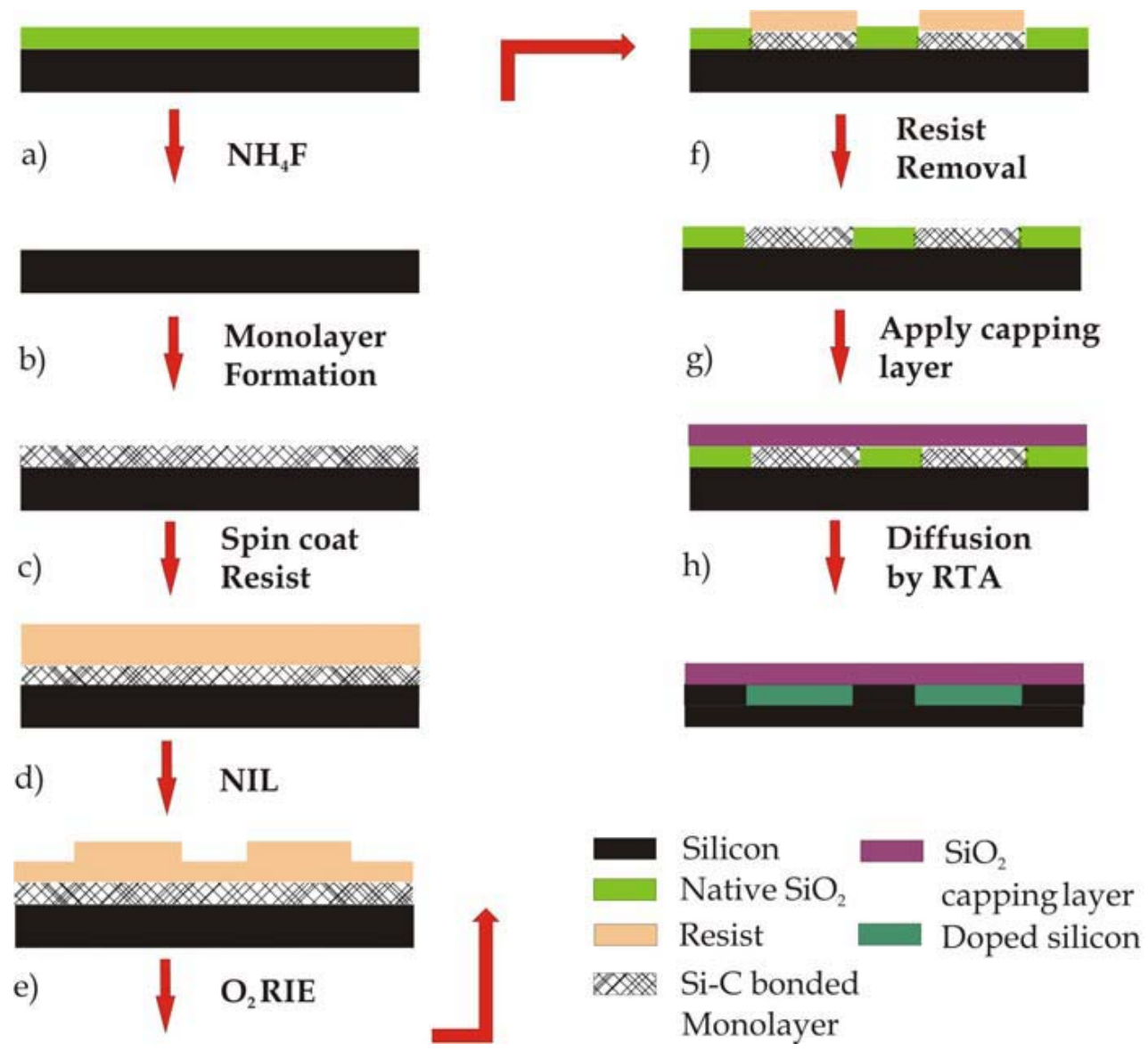

Scheme 6.2 Process scheme for the fabrication of locally doped silicon structures. a) Native $\mathrm{SiO}_{2}$ is fully stripped by $\mathrm{NH}_{4} \mathrm{~F}$. b) Monolayer formation using an organic - dopant atomcontaining - molecular precursor. c) Spin-coating of the imprint resist. d) Nanoimprint lithography. e) Reactive ion etching (RIE) of the residual layer and local removal of the monolayer using $\mathrm{O}_{2}$ plasma, with concomitant oxidation of the exposed Si areas. f) Resist removal by ultrasonication in acetone. g) Deposition of a $\mathrm{SiO}_{2}$ capping layer by e-beam evaporation. h) Rapid thermal annealing (RTA) to distribute the dopant atoms.

Our approach starts with the inverse NIL-patterning method as shown in Scheme 6.1B. In the first step (a) of the process (Scheme 6.2), the sample is fully stripped from its native oxide by $\mathrm{NH}_{4} \mathrm{~F}$. In step (b), a full organic monolayer is formed containing dopant atoms. In the next step, imprint resist is spin-coated on the preformed monolayer (c). The sample is patterned using nanoimprint lithography in step (d), creating micron size patterns 
suitable for inspection by SIMS imaging. At the same time, using NIL holds the promise for straightforward extension of the process to the sub-100 $\mathrm{nm}$ range by using high resolution molds. ${ }^{[26]}$ Reactive ion etching (RIE) using $\mathrm{O}_{2}$ plasma is used to remove the residual layer and the monolayer underneath in step (e). Resist removal by acetone in step (f) removes residual polymer, which results in pattern transfer. Further sample processing (steps $(\mathrm{g})$ and (h)) proceed according to the procedure reported by Javey et al. ${ }^{[6]}$ The specific route ${ }^{[26]}$ in Scheme 6.2 was selected to exclude formation of monolayers on $\mathrm{SiO}_{2}$-covered areas by reaction with the phosphonate capping group. ${ }^{[27]}$

Diffusion of the dopant impurities was achieved by RTA for 5 min at $1000{ }^{\circ} \mathrm{C}$ (Scheme 6.2h). At this temperature the organic monolayer fully disintegrates and the phosphorus dopant atoms diffuse into the silicon. To analyze the distribution of $\mathrm{P}$ dopant atoms in our patterned sample, we used TOF-SIMS for imaging and depth profiling (3D). In Figure 6.5, each image represents a $500 \times 500 \mu \mathrm{m}^{2}$ area. 

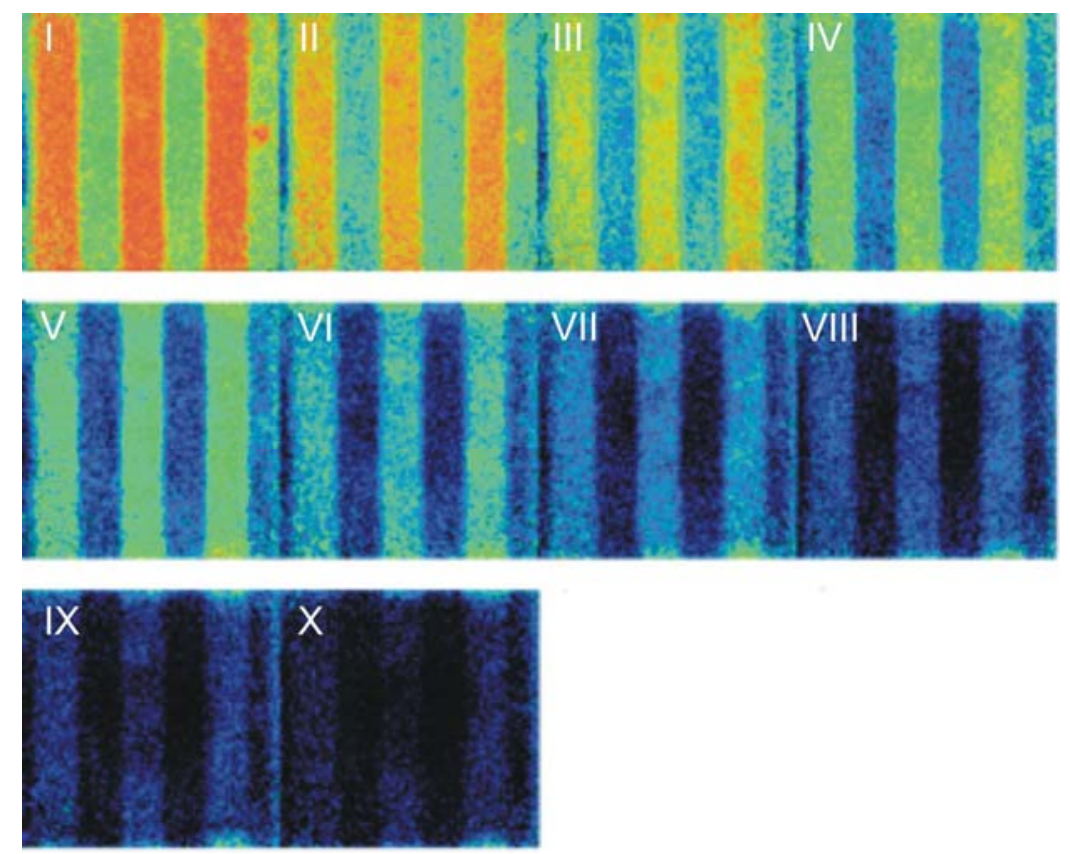

Figure 6.5 Phosphor elemental images recorded with TOF-SIMS (normalized to Si, image size $500 \times 500 \mathrm{~m}^{2}$ ) of a $1 \times 1 \mathrm{~cm}^{2}$ intrinsic $\mathrm{Si}(100)$ sample containing $100 \mu \mathrm{m}$-wide phosphorus-doped regions at $200 \mu \mathrm{m}$ period (RTA, $5 \mathrm{~min}$ at $1000^{\circ} \mathrm{C}$ ) at successive depths: each image represents an interval of $\sim 10 \mathrm{~nm}(0-10$ for image I, 10-20 for image II, etc.).

Integration of these images resulted in lateral line profiles, which are shown in Figure 6.6. The modulation, calculated by the ratio of high vs. low intensity of the signal, shows a value of $\sim 2.5$ in the top $0-10 \mathrm{~nm}$ which gradually increases up to $\sim 4$ from $40-100 \mathrm{~nm}$ depth. In Figure 6.6, the upper (red) curve corresponds to integration of the top left image in Figure 6.5. The dopant surface dose on a doped area measured $(2.3 \pm 0.1) \times 10^{19} \mathrm{P}$ atoms.cm ${ }^{-3}$ corresponding to an areal dose on a doped area of $N_{0, \text { line }}=(5.6 \pm 0.1) \times 10^{13} \mathrm{P}$ atoms.cm ${ }^{-2}$ (Figure 6.7a). The exact location of the spot was determined by optical interferometry (Figure 6.7b). In comparison, on an unpatterned sample that was doped by a full monolayer (RTA, 5 $\min$ at $\left.1000{ }^{\circ} \mathrm{C}\right)$, the dopant surface dose after SIMS depth profiling measured $(5.4 \pm$ $0.1) \times 10^{19} \mathrm{P}$ atoms.cm ${ }^{-3}$ which results in an areal dose $N_{0, \text { full sample }}=(1.1 \pm 0.1) \times 10^{14} \mathrm{P}$ atoms.cm ${ }^{-2}$. 


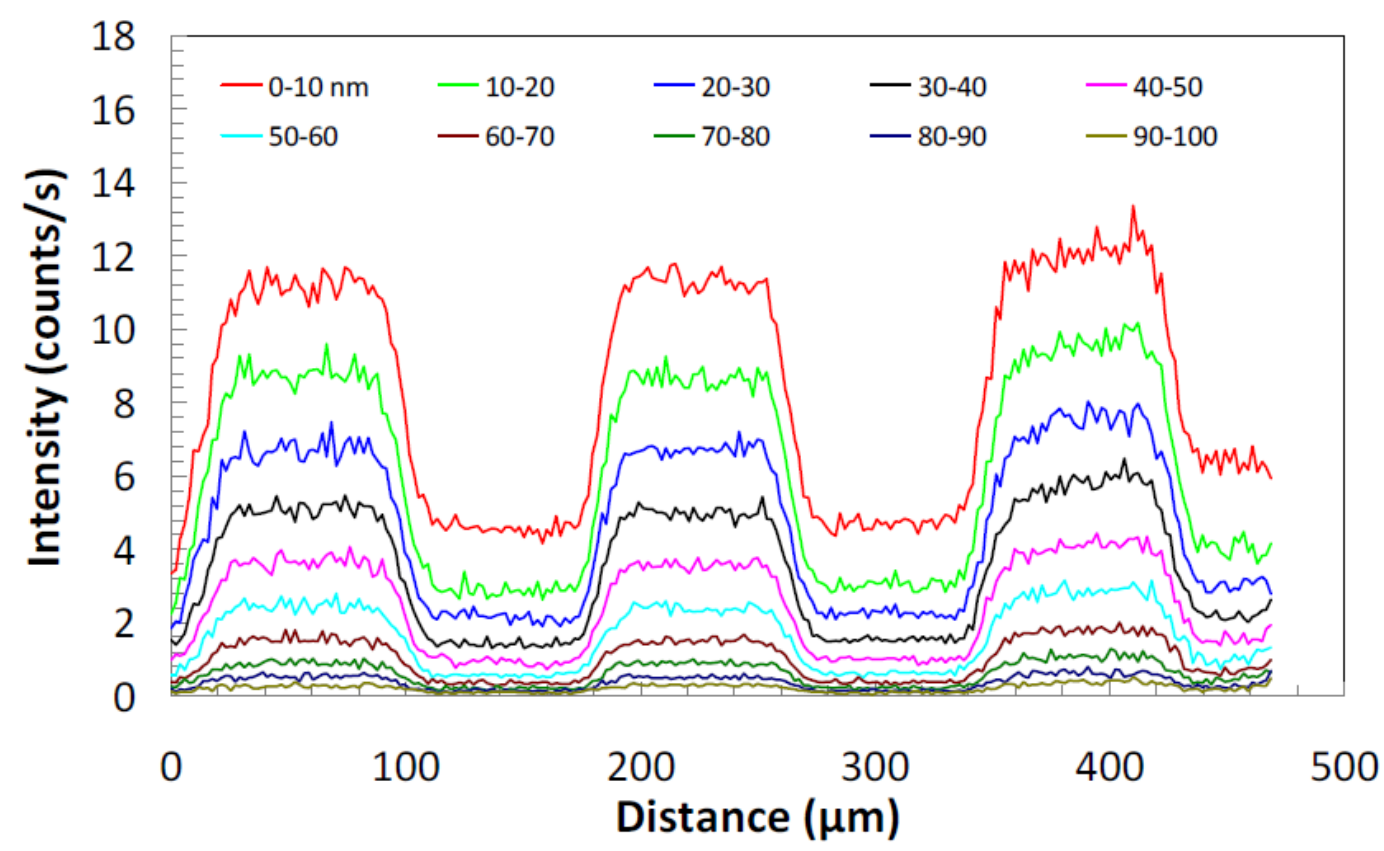

Figure 6.6. Intensity profiles in horizontal direction over the 10 images depicted in Figure 6.5 (integrated in vertical direction. Top 30 and bottom 30 pixels excluded).

A clear resistance difference is observed (Figure 6.8) between the current measured perpendicular (black curve) and parallel (red curve) to the line orientation. As expected, the perpendicular orientation resulted in the lowest resistance, since the doped lines form a parallel resistor network in this case. With the contacts oriented parallel to the lines we find a significantly larger resistance (resistors in series). 
a)
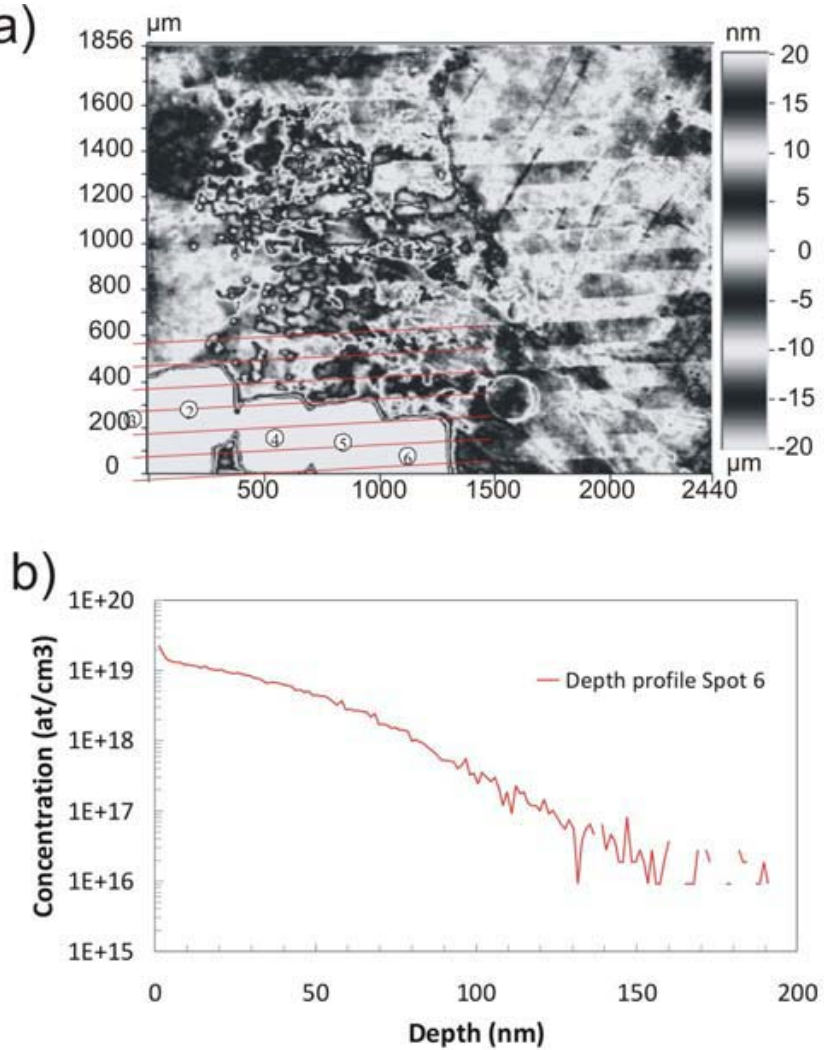

Figure 6.7. a) Optical profilometry of a sputtered region, b) SIMS depth profile

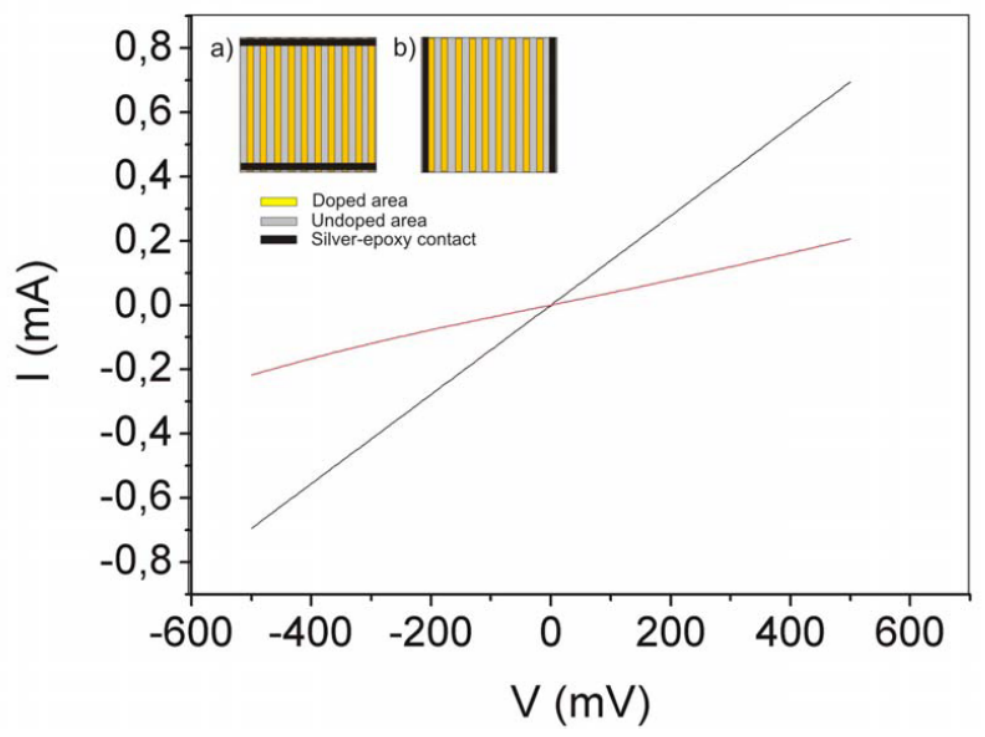

Figure 6.8. $I-V$ measurements on a $1 \times 1 \mathrm{~cm}^{2}$ intrinsic $\mathrm{Si}(100)$ sample containing phosphorus, n-doped line-shaped regions (width $100 \mu \mathrm{m}$, spacing $100 \mu \mathrm{m}$ ), using RTA, 5 min at $1000{ }^{\circ} \mathrm{C}$. For electrical characterization, silver epoxy contact pads were applied perpendicular (black curve, inset a)) or parallel (red curve, inset b)) to the line orientation. 
Hall measurements can provide useful information about the type (electrons or holes) and quantity of the (majority) charge carriers. Deduction of the charge carrier type (Figure $6.9 \mathrm{a} / \mathrm{b}$ ) revealed electrons as the majority charge carriers, as expected for $\mathrm{P}$ implantation. In case of a patterned sample, an average sheet carrier density $n_{s, a v}=(1.9 \pm$ $0.1) \times 10^{13} \mathrm{~cm}^{-2}$ was deduced from the Hall curve in Figure $6.9 \mathrm{a}$ at $T=300 \mathrm{~K}$. It must be noted that in the electrical determination of the majority carrier density is averaged over doped and undoped areas on the whole sample, while SIMS depth profiling can be performed on specific locations. As the ratio of doped to undoped areas is $1: 1$ an estimation for the sheet density on the doped areas of the sample can be obtained by multiplication of the average value by a factor 2 which results in $n_{s, f u l l}=(3.8 \pm 0.1) \times 10^{13} \mathrm{~cm}^{-2}$. At $T=150 \mathrm{~K}$ we determined an average sheet density $n_{s, a v}=(9.4 \pm 0.1) \times 10^{12} \mathrm{~cm}^{-2}$ from the curve in Figure $6.9 \mathrm{~b}$ which results in $n_{s, f u l l}=(1.9 \pm 0.1) \times 10^{13} \mathrm{~cm}^{-2}$.

The $300 \mathrm{~K}$ value $\left(n_{s, f u l l}=(3.8 \pm 0.1) \times 10^{13} \mathrm{~cm}^{-2}\right)$ agrees well with the local areal dose as determined by SIMS depth profiling $\left(N_{0, \text { line }}=(5.6 \pm 0.1) \times 10^{13} \mathrm{P}\right.$ atoms.cm $\left.{ }^{-2}\right)$.

Since the maximum amount of dopant atoms that can diffuse into the silicon is determined by the self-limiting character of organic monolayers, scaling is also expected in terms of sheet resistance Rs. For a fully monolayer-doped, but unpatterned sample we determined $R_{s}=(7.6 \pm 0.1) \times 10^{2} \Omega / \square$. The average sheet resistance of our patterned sample should naturally be larger and we found $R_{S}=(1.5 \pm 0.1) \times 10^{3} \Omega / \square$. Since the ratio of line width and spacing is 1:1, also the sheet resistances (by 4-point probe measurements) are expected to scale accordingly. $\left(R_{S \text {, unpatterned }} / R_{s, \text { patterned }}\right)=0.5$, which corresponds to the expected scaling using a limited source condition such as a molecular monolayer. For an estimated depth of $100 \mathrm{~nm}$ for the electrically conductive layer this results in a (bulk) resistivity $\rho=(1.5 \pm 0.1) \times 10^{-2} \Omega . \mathrm{cm}$ for an unpatterned sample, and $\rho=(7.6 \pm 0.1) \times 10^{-3} \Omega . \mathrm{cm}$ for our patterned sample. 
a)

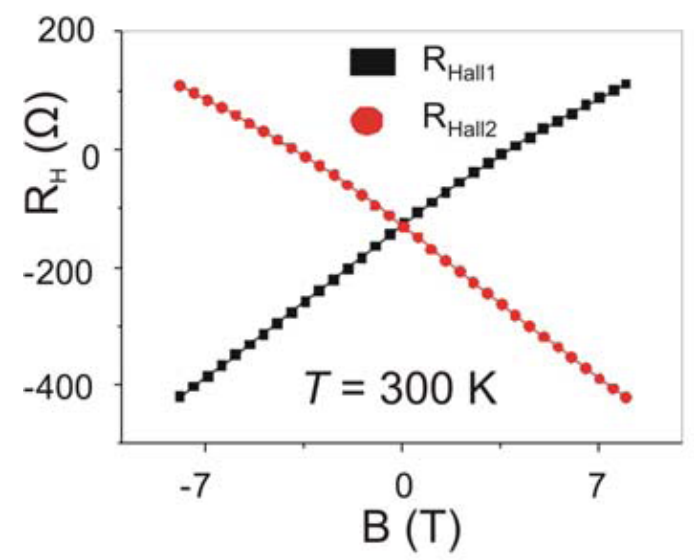

c)

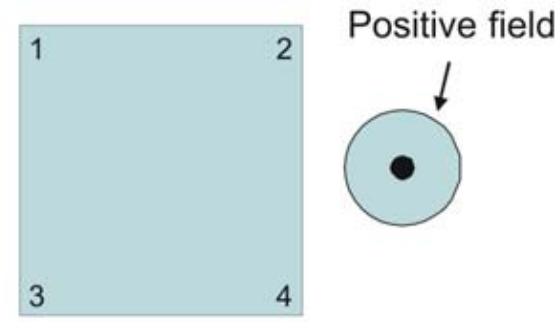

$R_{\text {hor: }}$ current $1(+)->2(-)$, voltage $3(+)->4(-)$

$\mathrm{R}_{\text {ver }}$ current $1(+)->3(-)$, voltage $2(+)->4(-)$

Hall ${ }_{1}$ : current $3(+)->2(-)$, voltage $1(+)->4(-)$

Hall ${ }_{2}$ : current $1(+)->4(-)$, voltage $3(+)->2(-)$ b)

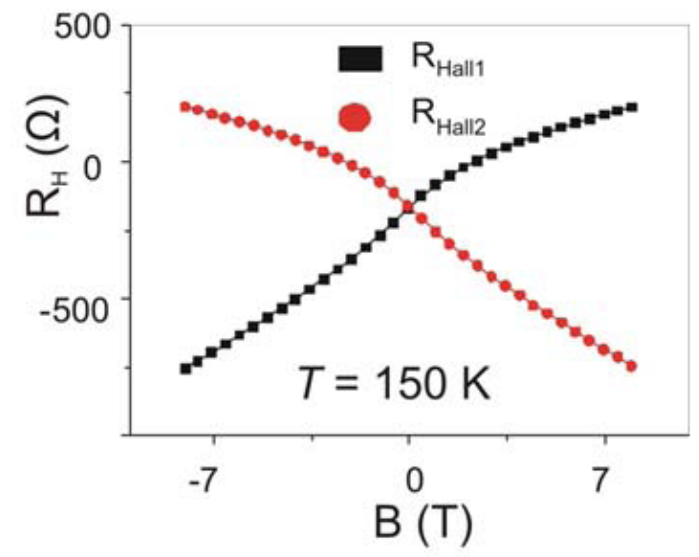

Figure 6.9. a) Hall resistance measurements at $T=300 \mathrm{~K}$ on a $1 \times 1 \mathrm{~cm}^{2}$ intrinsic Si (100) patterned sample phosphorus, n-doped. (RTA, $5 \mathrm{~min}$ at $1000{ }^{\circ} \mathrm{C}$ ). b) Hall resistance measurements at alternating negative and positive field on a $1 \times 1 \mathrm{~cm}^{2}$ intrinsic Si (100) patterned phosphorus n-doped sample at $T=150 \mathrm{~K}\left(\mathrm{RTA}, 5 \mathrm{~min}\right.$ at $1000{ }^{\circ} \mathrm{C}$ ) c) Probe configurations used in Van der Pauw and Hall measurements.

The calculated mobility of the majority carrier $\left(\mu_{m}\right)$ at $T=300 \mathrm{~K}$ for our patterned sample was $(2.3 \pm 0.1) \times 10^{2} \mathrm{~cm}^{2} \cdot \mathrm{V}^{-1} \cdot \mathrm{s}^{-1}$ and increased to $(3.5 \pm 0.1) \mathrm{cm}^{2} \cdot \mathrm{V}^{-1} \cdot \mathrm{s}^{-1}$ at $T=150 \mathrm{~K}$. Figure 6.10 shows a plot of the sheet resistance $R_{\mathrm{s}}$ versus temperature for a patterned sample. 


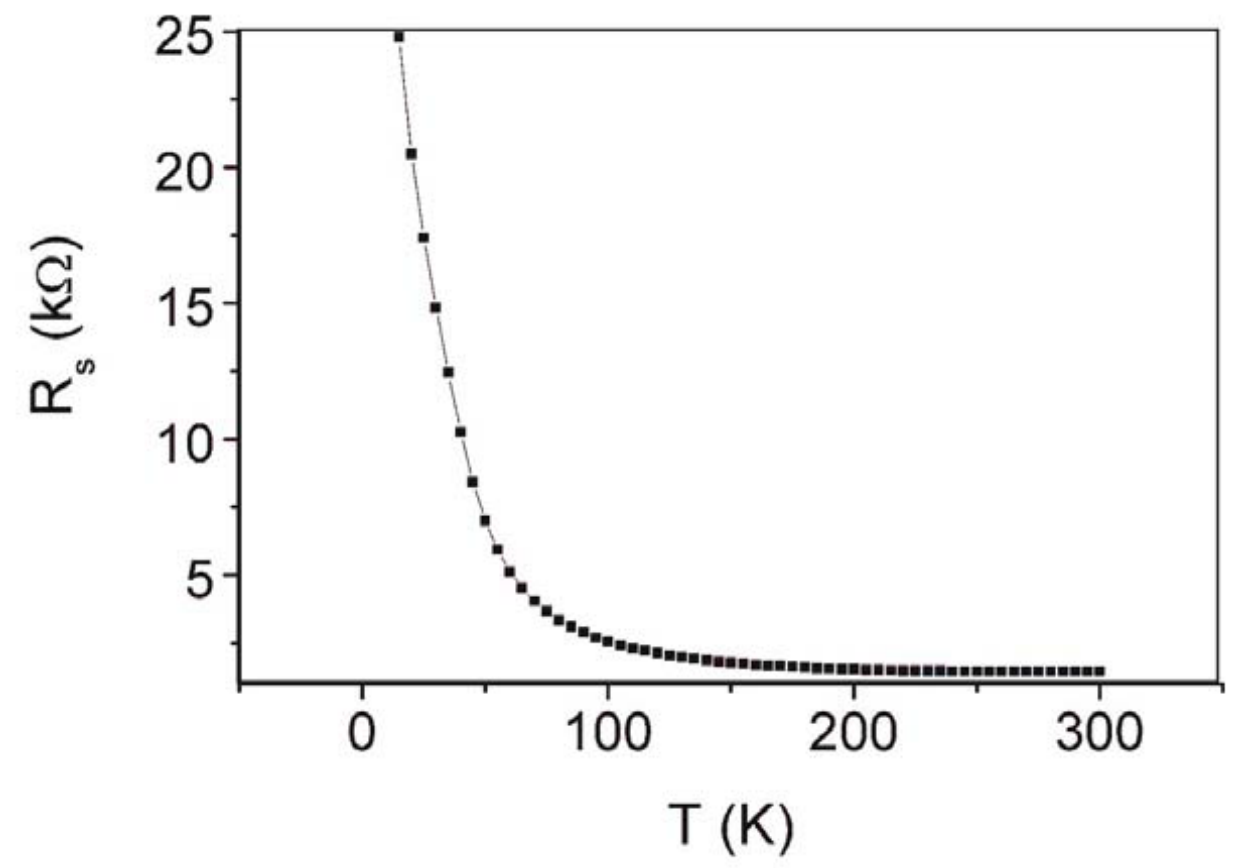

Figure 6.10. Sheet resistance $R s$ vs. $T$ by Van der Pauw measurements of a patterned Si sample with $100 \mu \mathrm{m}$ P-doped line patterns and $100 \mu \mathrm{m}$ spacing.

For an estimation of the doping efficiency we use the maximum attainable surface concentration of a full monolayer as determined in the section 6.5 measurement part $[(2.2 \pm$ $0.1) \times 10^{14} \mathrm{P}$ atoms.cm ${ }^{-2}$. The ratio of the SIMS areal dose to surface concentration $\mathrm{x} 100 \%$ is the doping efficiency. The areal doses determined by SIMS for a doped line $N_{0 \text {, line }}=(5.6 \pm$ $0.1) \times 10^{13} \mathrm{P}$ atoms.cm ${ }^{-2}($ Figure $6.7 \mathrm{a} / \mathrm{b})$ and $N_{0, \text { full }}=(1.1 \pm 0.1) \times 10^{14} \mathrm{P}$ atoms.cm ${ }^{-2}$ measured on a fully monolayer doped, unpatterned sample were used in the calculation. This results in doping efficiencies of $26 \%$ for a doped line and $50 \%$ on a full sample. 


\subsection{Conclusions}

We have developed two schemes for alkyl monolayer pattern fabrication on oxidefree, hydrogen-terminated silicon. To this purpose, top-down NIL was combined with bottom-up formation of molecular monolayers via hydrosilylation. Patterns with feature sizes ranging from $100 \mathrm{~nm}$ to $100 \mu \mathrm{m}$ have been faithfully reproduced. Feature sizes attainable using this method are, in principle, only limited by the resolution of the NIL mold and by the faithful pattern transfer in the subsequent etching and functionalization steps.

Our work also shows it is possible to fabricate highly doped regions in silicon using a combination of nanoimprint lithography and organic molecular monolayers. By demonstrating proof-of-principle of lateral control over positioning of dopants using molecular monolayer formation we imagine our work can contribute to fabrication of electronic circuitry and devices such as diodes, FETs or nanowires.

\subsection{Acknowledgement}

The major part of the work presented in this chapter was performed in collaboration with W. Pim Voorthuijzen. Alberto Gomez-Casado is acknowledged for performing the AFM measurements. Y. Zhao is acknowledged for preparation of the 100-nm ridge mold. Dr. J.G.M. van Berkum (MiPlaza Materials Analysis, Eindhoven, the Netherlands) is acknowledged for SIMS characterization.

\subsection{Experimental section}

Materials. All chemicals were purchased from commercial sources and used as received. Silicon (111) (p-type) was used for patterning experiments. Nearly intrinsic Si (100) (4”, thickness $380 \mu \mathrm{m}, \mathrm{FZ}$, resistivity between 24.4 and $42.5 \mathrm{k} \Omega . \mathrm{cm}^{-1}$ was purchased from University Wafer, Boston (MA), USA. Solvents for monolayer formation, rinsing, and $\mathrm{SiO}_{2}$ 
etching were deoxygenated by five repetitive cycles of stirring the solution under vacuum followed by argon sparging. Milli-Q water (resistivity>18 $\mathrm{M} \Omega . \mathrm{cm}$ ) was used in all experiments. 1-Undecenyl-dimethylphosphonate was synthesized according to a literature procedure $^{[28]}$.

\section{Synthesis of alkene-terminated BODIPY derivative 1, (4,4-Difluoro-8-(4-(10-} undecenyloxy))phenyl-1,3,5,7-tetramethyl-2,6-diethyl-4-bora-3a,4a-diaza-s-indacene).

The synthesis of 4-(10-undecenyloxy)benzaldehyde has been described previously. ${ }^{[29]} 3$ Ethyl-2,4-dimethyl pyrrole (5.26 mmol, $648 \mathrm{mg}$ ) and 4-(10 undecenyloxy)benzaldehyde (2.6 mmol, $713 \mathrm{mg}$ ) were dissolved in $200 \mathrm{~mL}$ of dry $\mathrm{CH}_{2} \mathrm{Cl}_{2}$ under argon atmosphere. One drop of trifluoroacetic acid (TFA) was added, and the solution was stirred at room temperature for 3-4 h. At this point, a solution of 2,3-dichloro-5,6 dicyanobenzoquinone (DDQ) (2.6 mmol, $640 \mathrm{mg}$ ) in $50 \mathrm{~mL}$ dry $\mathrm{CH}_{2} \mathrm{Cl}_{2}$ was added, and stirring was continued for $30 \mathrm{~min}$ followed by the addition of $3 \mathrm{~mL} \mathrm{Et}_{3} \mathrm{~N}$ and $3 \mathrm{~mL} \mathrm{BF}_{3}$. $\mathrm{OEt}_{2}$. After stirring for $30 \mathrm{~min}$, the reaction mixture was washed three times with water and dried over $\mathrm{Na}_{2} \mathrm{SO}_{4}$. The solvent was evaporated, and the residue was purified by silica gel column chromatography $\left(1: 1 \mathrm{CH}_{2} \mathrm{Cl}_{2}\right.$ :hexanes $)$ to give a red solid (374 mg, 30\%)

${ }^{1} \mathrm{H}$ NMR $\left(300 \mathrm{MHz}, \mathrm{CDCl}_{3}\right) \delta 7.14(\mathrm{~d}, J=8.7 \mathrm{~Hz}, 2 \mathrm{H}), 6.98(\mathrm{~d}, J=8.7 \mathrm{~Hz}, 2 \mathrm{H}), 5.88-5.75(\mathrm{~m}$, 1H), 5.03-4.86 (m, 2H), $4.01(\mathrm{t}, J=6.6 \mathrm{~Hz}, 3 \mathrm{H}), 2.52(\mathrm{~s}, 6 \mathrm{H}), 2.30(\mathrm{q}, J=7.5 \mathrm{~Hz}, 4 \mathrm{H}), 2.05$ $(\mathrm{q}, J=7.2 \mathrm{~Hz}, 2 \mathrm{H}), 1.83(\mathrm{p}, J=6.9 \mathrm{~Hz}, 2 \mathrm{H}), 1.47-1.25(\mathrm{~m}, 24 \mathrm{H}){ }^{13} \mathrm{C} \mathrm{NMR}\left(75 \mathrm{MHz}, \mathrm{CDCl}_{3}\right) \delta$ $159.7,153.6,140.6,139.4,138.7,132.8,131.4,129.6,127.8,115.1,114.3,68.3,34.0,29.9$, 29.7, 29.6, 29.5, 29.3, 29.1, 26.3, 17.3, 14.9, 12.7, 12.1 MS (ESI) calcd for $\mathrm{C}_{34} \mathrm{H}_{47} \mathrm{BF}_{2} \mathrm{~N}_{2} \mathrm{O}$ $(\mathrm{M}+\mathrm{H})$ 549.37; found 549.6.

Substrate and monolayer preparation. Si substrates were cleaned by immersion in Piranha solution (concentrated $\mathrm{H}_{2} \mathrm{SO}_{4}: 33 \% \mathrm{H}_{2} \mathrm{O}_{2}=3: 1 \mathrm{v} / \mathrm{v}$. CAUTION! Piranha solutions should be handled with great care in open containers in a fume hood. Piranha is highly corrosive and 
toxic and potentially explosive.) for $30 \mathrm{~min}$. Cleaned substrates were rinsed with Milli-Q water and blown dry in a stream of nitrogen. A hydrogen-terminated silicon (111) surface was generated by immersion of the substrate in an argon-sparged $40 \mathrm{wt} \%$ aqueous $\mathrm{NH}_{4} \mathrm{~F}$ solution for 15 min followed by extensive rinsing with Milli-Q water and drying in a stream of nitrogen.

Monolayers were assembled on Si (111) using the literature procedure of Sieval et al. ${ }^{[17]}$ with minor changes. Monolayers were formed in all experiments using diluted reagents as the molecular precursor in refluxing mesitylene $\left(b p=166{ }^{\circ} \mathrm{C}\right)$ as the solvent. Glassware was predried with a heat gun at $200{ }^{\circ} \mathrm{C}$ for 15 min. A small three-necked flask was equipped with a reflux cooler. The other inlets were sealed with a rubber septum. A long needle serving as the nitrogen inlet was inserted through the septum in one of the inlets. Under vigorous $\mathrm{N}_{2}$ flow, freshly hydrogen-terminated samples were inserted. Deoxygenated reagents and solvents were taken up through a septum by a syringe and subsequently transferred to the reaction vessel, after which the needle was placed just above the solution. The nitrogen flow was reduced to a minimum to reduce evaporation of liquids. A metal bath at $180{ }^{\circ} \mathrm{C}$ was used to heat the flask containing the samples. After monolayer formation $(2.5 \mathrm{~h}$ for the hexadecyl functionalized layers, and overnight for fluorescent molecule 1), the samples were rinsed with ethanol, acetone, and Milli-Q water and ultrasonicated for $15 \mathrm{~min}$ in acetone to remove physisorbed material.

Nanoimprint lithography. Silicon molds for NIL were prepared by e-beam writing or by using standard photolithographic methods followed by RIE using an Electrotech Twin system PF 340. Mold 1 consisted of a hexagonal pattern of $100 \mu \mathrm{m}$ pillars in diameter with a height of $450 \mathrm{~nm}$. Mold 2 consisted of $3 \mu \mathrm{m}$ ridges at $8 \mu \mathrm{m}$ period with a height of $550 \mathrm{~nm}$. Mold 3 was purchased from NIL Technology, Denmark, which was a nickel-plated mold and consisted of a square array of $150 \mathrm{~nm}$ pillars in diameter at $500 \mathrm{~nm}$ period with a height of 
$100 \mathrm{~nm}$. Mold 4 consisted of $100 \mathrm{~nm}$ wide ridges at alternating periods of 3 and $5 \mu \mathrm{m}$ with a feature height of $110 \mathrm{~nm}$ made by edge lithography. ${ }^{[22]}$ Mold 5 consisted of $100 \mu \mathrm{m}$ wide trenches at $200 \mu \mathrm{m}$ period with a height of $125 \mathrm{~nm}$. Before use, molds 1, 2, 4, and 5 were coated with an antisticking layer by gas phase deposition of $1 \mathrm{H}, 1 \mathrm{H}, 2 \mathrm{H}, 2 \mathrm{H}$ perfluorodecyltrichlorosilane (PFDTS) ${ }^{[30]}$ overnight followed by a 30 min bake at $120{ }^{\circ} \mathrm{C}$. Polymerized materials were removed from the silanized silicon molds by ultrasonication for 5 $\min$ in acetone.

A resist layer of PMMA $(\mathrm{Mw}=350 \mathrm{kD})$ (molds 1, 2, 4 and 5) or commercially available resist (mr-I 7020E, Micro Resist Technology, GmbH) (mold 3) was applied by spin coating on a cleaned $\mathrm{Si} / \mathrm{SiO}_{2}$ surface (Scheme 5.1A) or on a preformed monolayer on $\mathrm{Si}$ (Scheme 5.1B).

For imprinting, mold and substrate were contacted at 40 bar pressure using a handpress (Specac) at $180{ }^{\circ} \mathrm{C}(\mathrm{PMMA})$ or at $130{ }^{\circ} \mathrm{C}(\mathrm{mr}-\mathrm{I} 7020 \mathrm{E})$. Residual layer removal was achieved by (anisotropic) RIE on a Electrotech Twin system PF 340 using $\mathrm{O}_{2}$ plasma.

Doping of silicon by molecular monolayers was achieved as previously described ${ }^{[6]}$. A thin film of $\mathrm{SiO}_{2}(\sim 100 \mathrm{~nm})$ was deposited by e-gun evaporation of $\mathrm{SiO}_{2}$ pellets of $1-5 \mathrm{~mm}$ in diameter (Kurt Lesker) using a Balzers BAK 600 apparatus operating at a base pressure of 2e-6 bar. By RTA the distribution of the dopant atoms was achieved using an Amtech Tempress Omega Junior 2-stack oven at $1000{ }^{\circ} \mathrm{C}$ for $5 \mathrm{~min}$ in a $\mathrm{N}_{2^{-}}$atmosphere. After cool down of the samples the template $\mathrm{SiO}_{2}$ layer was stripped off using buffered $\mathrm{HF}$.

\section{Measurements}

Water contact angles were determined using a Kruss Contact Angle Measurement System G 10, and the data was processed using the Drop Shape Analysis 1.51 program. Contact angle measurements were taken on three different points on the sample, and the results were averaged. Resist film thicknesses were measured using a Plasmos SD 2002 ellipsometer at $\lambda$ 
$=632.8 \mathrm{~nm}$. XPS was performed using a Physical Electronics Quantera SXM equipped with a $\mathrm{Al} \mathrm{K} \alpha$ monochromatic excitation source (source energy $=1486.6 \mathrm{eV}$, take-off angle set at $45^{\circ}$ ). SXI images were collected using these settings or using an X-ray beam down to $4.5 \mathrm{~W}$ per $18 \mu \mathrm{m}$. The X-ray beam was set to $1 \mathrm{~W}$ per $9 \mu \mathrm{m}$ for line scans (Figure 6.1c) across 4.5 dots for $16 \mathrm{~h}\left(298 \mathrm{~K},<8 \times 10^{-9}\right.$ Torr $)$. SIMS measurements were performed using a Cameca ims6f magnetic-sector SIMS and an Ion-Tof TOF-SIMS IV instrument. The Cameca was operated in positive mode with $3 \mathrm{keV} \mathrm{O}_{2}^{+}$primary ions and additional $\mathrm{O}_{2}$-flooding to enhance the $\mathrm{P}^{+}$secondary ion yield. The TOF-SIMS was also operated in positive mode and $\mathrm{O}_{2}$ flooding; the primary ions were $2 \mathrm{keV} \mathrm{O}_{2}^{+}$for sputtering and $25 \mathrm{keV} \mathrm{Bi}^{+}$for analysis.

The samples have also been inspected using optical profilometry to measure crater depths for depth scale calibration and to visualize the line pattern.

Images in Figure 6.5 were collected after sputtering of the top $2 \mathrm{~nm}$ to remove redeposited physisorbed materials. The utmost edges of the images should be ignored, since they are sputtered slower, resulting in erroneously higher $\mathrm{P}$ concentration.

Determination of the Hall-coefficient was done using a Quantum Design Physical Property Measurement System (PPMS) using PPMS Multivu software. The system was configured with a $8 \mathrm{~T}$ strength longitudinal magnet. Measurements were conducted at temperatures from $293 \mathrm{~K}$ down to $10 \mathrm{~K}$. Samples were subjected to wafer bonding for attachment to the sample holder for measurements.

Introduction of impurities with a higher atomic valence than silicon in the crystal lattice is expected to result in a majority of charge-carriers which is negative $\left(R_{H}<0\right)$. Figure $6.9 \mathrm{c}$ shows our used setup and configuration of measurement probes. For the black curve in Figure $6.9 \mathrm{a} / \mathrm{b}$ ) the configuration of the probes is as follows: $3(+), 2(-), 1(+), 4(-)$. The current flows from contact 3 to contact 2 because of the higher potential of contact 3 . Application of the right-hand rule shows that the Lorentz force tends to accumulate electrons at contact 1 at 
positive field. This effect causes an increase in potential at contact 1 . Measurement of the voltage difference between contacts 1 and 4 should therefore result in a positive Hall coefficient since the voltage difference (V1-V4) is also positive. This is exactly what is expected for electrons as the majority charge carrier.

For resistance measurements over parallel lines (Figure 3, inset) contact pads were applied along a $1 \times 1 \mathrm{~cm}^{2}$ sample perpendicular to the pattern direction using a silver epoxy paste. A thin line of paste of approximately $1 \mathrm{~mm}$ in width was applied along the edge of each side of the sample on the hydrogen-terminated silicon surface. The samples were shortly annealed at $120{ }^{\circ} \mathrm{C}$ for 30 minutes and stored overnight. Resistances over the samples were determined using a 2-point probe IV-measurement using a Keithley 4200 Semiconductor characterization system. In our case, using a $0.9 \times 0.9 \mathrm{~cm}^{2}$ mold for imprint approximately 45 parallel wires were measured of $100 \mu \mathrm{m}$ in width separated by $100 \mu \mathrm{m}$ spacings. As a control measurement the contact pads were stripped by immersion of the samples for $30 \mathrm{~min}$ in fuming $\mathrm{HNO}_{3}$ and re-applied parallel to the direction of our doped pattern after which the resistance over the sample in opposite direction was determined.

For the determine the dopant surface dose, the maximum number of $=\mathrm{SiH}_{2}$ sites available on the Si (100) surface based on the dimension of a unit cell $\left(14.74 \AA^{2}\right)^{[21]}$. Based on this value a molecular density of $6.7^{*} 10^{14}$ molecules. $\mathrm{cm}^{-2}$ is calculated. However, only a maximum of $61 \%$ surface coverage can be obtained due to sterical constraints of the alkyl chains. The maximum molecular density which can be obtained on $\mathrm{Si}(100)$ therefore is $4.1 * 10^{14}$ molecules. $\mathrm{cm}^{-2}$. Such an ideal coverage of the surface only proceeds when using long chain alkyl terminated monolayers and Faucheux et al. ${ }^{[31]}$ determined for acid-terminated (-COOH) undecenyl moieties a surface coverage of 0.32 on $\mathrm{Si}$ (111). Therefore, it is expected that for monolayers prepared using 1-undecenyl-dimethylphosphonate, a compound with an even bulkier head group, the surface coverage will not extend 0.32 . Therefore, based on this 
surface coverage factor we estimate a maximum molecular density of $2.2^{*} 10^{14}$ molecules.cm ${ }^{-}$

2. With a ratio of one phosphorus atom per precursor molecule the molecular density can directly be compared to the areal doses extracted from SIMS measurements to obtain the doping efficiency.

\section{Fluorescence microscopy}

Fluorescence microscope images were taken using an Olympus inverted research microscope IX71 equipped with a mercury burner U-RFL-T as light source and a digital Olympus DR70 camera for image acquisition. Blue excitation $\left(450 \mathrm{~nm} \leq \lambda_{\mathrm{ex}} \leq 480 \mathrm{~nm}\right)$ and green emission $\left(\lambda_{\mathrm{em}} \geq 515 \mathrm{~nm}\right)$ was filtered using a Dapi Olympus filter cube. All fluorescence microscopy images were acquired in air.

\section{Atomic force microscopy (AFM)}

AFM imaging was performed using an AFM Nanoscope III, (Veeco Digital Instruments, U.S.A.) in contact mode equipped with a $\mathrm{Si}_{3} \mathrm{~N}_{4}$ tip with a J-scanner at a scan rate of $1.5 \mathrm{~Hz}$.

\subsection{References}

[1] P. S. Peercy, Nature 2000, 406, 1023-1026.

[2] M. Lundstrom, Science 2003, 299, 210-211.

[3] H. J. Queisser; E. E Haller, Science 1998, 281, 945-950.

[4] D. H. Lee; J. W. Mayer, Proc. IEEE 1974, 62, 1241-1255.

[5] A. Renau, Nucl. Instr. Meth. Phys. Res. B 2005, 237, 284-289.

[6] J. C. Ho, R. Yerushalmi, Z. A. Jacobson, Z. Fan, R. L. Alley, A. Javey, Nat. Mater. 2008, 7, 62-67.

[7] J. M. Buriak, Chem. Commun. 1999, 1051-1060. 
[8] J. M. Buriak, Chem. Rev. 2002, 102, 1271-1308.

[9] R. T. Lee, R. I. Carey, H. A. Biebuyck, G. M. Whitesides, Langmuir 1994, 10, 741-749.

[10] F. Effenberger, G. Gotz, B. Bidlingmaier, M. Wezstein, Angew. Chem. Int. Ed. 1998, $37,2462-2464$.

[11] J. T. C. Wojtyk, M. Tomietto, R. Boukherroub, D. D. M. Wayner, J. Am. Chem. Soc. 2001, 123, 1535-1536.

[12] P. T. Hurley, A. E. Ribbe, J. M. Buriak, J. Am. Chem. Soc. 2003, 125, 11334-11339.

[13] H. Mizuno, J. M. Buriak, J. Am. Chem. Soc. 2008, 130, 17656-17657.

[14] B. Klingebiel, L. Scheres, S. Franzka, H. Zuilhof, N. Hartmann, Langmuir 2010, 26, $6826-6831$.

[15] L. J. Guo, J. Phys. D: Appl. Phys. 2004, 37, R123-R141.

[16] S. Y. Chou, P. R. Krauss, P. J. Renstrom, Science 1996, 272, 85-87.

[17] A. B. Sieval, V. Vleeming, H. Zuilhof, E. J. R. Sudholter, Langmuir 1999, 15, 82888291.

[18] a) A. Ulman, Chem. Rev. 1996, 96, 1533-1554; b) F. Schreiber, Prog. Surf. Sci. 2000, 65, 151-256; c) X.-M. Li, J. Huskens, D. N. Reinhoudt, J. Mater. Chem. 2004, 14, $2954-2971$.

[19] M. R. Linford, P. Fenter, P. M. Eisenberger, C. E. D. Chidsey, J. Am. Chem. Soc. 1995, 117, 3145-3155.

[20] A. B. Sieval, A. L. Demirel, J. W. M. Nissink, M. R. Linford, J. H. van der Maas, W. H. de Jeu, H. Zuilhof, E. J. R. Sudholter, Langmuir 1998, 14, 1759-1768.

[21] A. B. Sieval, R. Linke, H. Zuilhof, E. J. R. Sudholter, Adv. Mater. 2000, 12, 14571460. 
[22] Y. Zhao, E. Berenschot, M. de Boer, H. Jansen, N. Tas, J. Huskens, M. Elwenspoek, J. Micromech. Microeng. 2008, 18, 064013.

[23] a) A. Lambacher, P. Fromherz, Appl. Phys. A: Mater. Sci. Process. 1996, 63, 207-216;

b) T. Lasseter, B. H. Clare, B. M. Nichols, N. L. Abbott, R. J. Hamers, Langmuir 2005, 21, 6344-6355.

[24] BODIPY is a registered trademark of Molecular Probes, Inc.

[25] M. D. Yilmaz, O. A. Bozdemir, E. U. Akkaya, Org. Lett. 2006, 8, 2871-2873.

[26] W. P. Voorthuijzen, M. D. Yilmaz, A. Gomez-Casado, P. Jonkheijm, W. G. Van der Wiel, J. Huskens, Langmuir 2010, 26, 14210.

[27] R. Yerushalmi, J. C. Ho, Z. Fan, A. Javey, Angew. Chem. Int. Ed. 2008, 47, 4440.

[28] R.T. Lee, R. I. Carey, H. A. Biebuyck, G. M. Whitesides, Langmuir 1994, 10, 741.

[29] G. S. Lee, Y.-J. Lee, S. Y. Choi, Y. S. Park, K. B. Yoon, J. Am. Chem. Soc. 2000, $122,12151-12157$.

[30] G. S. Ferguson, M. K. Chaudhury, H. A. Biebuyck, G. M. Whitesides, Macromolecules 1993, 26, 5870-5875.

[31] A. Faucheux, A. C. Gouget-Laemmel, C. H. de Villeneuve, R. Boukherroub, F. Ozanam, P. Allongue, J.-N. Chazalviel, Langmuir, 2006, 22, 153-162. 


\section{Chapter 7}

\section{Fabrication of Two-Dimensional Organic Spin Systems on Gold}

Using molecules carrying unpaired spins as elements of computer circuits, electronic and optical switches, sensors, and charge storage is a powerful new direction in the science and technology of nanometer-scale complex systems. In this chapter, the fabrication of monolayers of organic molecules with unpaired spins on a thin gold film is described. The systems investigated here consist of monolayers of two terpyridinyl-metal complexes and two different nitroxyl 2,2,6,6-tetramethylpiperidin-1-oxyl (TEMPO) radicals. Monolayers were characterized by XPS and cyclic voltammetry measurements, and showed the existence of unpaired spins on the gold substrate. Electrical characterization was performed by Van der Pauw measurements. In some systems with diluted monolayers, electrical transport measurements showed an increase of the gold film sheet resistance upon cooling down to below 20K, possibly implying Kondo physics. 


\subsection{Introduction}

The electronic interaction of magnetic impurities with their environment can lead to a spin phenomenon. This is a physical phenomenon and widely studied in condensed matter physics. The effect arises from the interactions between a single magnetic atom, such as cobalt, and the many electrons in an otherwise non-magnetic metal such as copper. Such an impurity typically has an intrinsic angular momentum or spin that interacts with all the surrounding electrons in the metal. The Kondo effect is named after the Japanese physicist Jun Kondo, who in 1964 gave an explanation for this physical phenomenon. ${ }^{[1-9]}$ This phenomenon is characterized by an anomalous temperature dependence of the resistivity. Usually, the electrical resistivity of a pure metal decreases as its temperature drops. This is because the lattice vibrations (phonons) diminish, and conduction electrons can travel more easily through the crystal. This decrease usually saturates at a certain temperature due to (static) impurities in the material, which cause scattering of the conduction electrons, even at the lowest attainable temperatures. A higher amount of impurities results in a higher saturation resistivity. However, De Haas et al. observed a resistivity increase of gold samples with decreasing temperature below $4 \mathrm{~K}$. This discovery was followed by many observations of increasing metal resistivity below a certain temperature. It was proven that this observed resistivity minimum was caused by the presence of diluted magnetic impurities, which are impurities with a local magnetic moment caused by the spin of unpaired electrons. ${ }^{[10]}$

In this chapter, we propose and investigate a potential Kondo system which consists of a thin gold layer, with on top a self-assembled monolayer (SAM) of molecules having an unpaired spin. Because of their unpaired spin these molecules have a net magnetic moment and therefore behave as magnetic impurities on top of the metal substrate. The 2D spin systems theoretically offer the opportunity to investigate the interaction between the magnetic molecules on top of the substrate and the conduction electrons inside the metal film. This 
interaction leads to a Kondo effect which can be macroscopically observable (typically through a resistance change at liquid helium temperatures). In order to observe the Kondo effect, electrons must be able to tunnel on and off the magnetic impurity molecules.

A schematic representation of a 2D spin system is given in Figure 7.1. It shows a thin metal film covered with a monolayer of organic molecules with unpaired spins. By varying the concentration of these molecules, the average distance between the unpaired spins may be varied. In this way the interaction between the unpaired spins (RKKY mechanism, the interaction of the spin of two magnetic ions) can be influenced. ${ }^{[11-13]}$ When the unpaired electrons are separated further, the RKKY coupling will decrease. Since a strong RKKY coupling locks the spins of the electrons, spin-flip scattering cannot occur anymore and the Kondo effect will be suppressed. This means that the $2 \mathrm{D}$ spin systems offer a method for studying the transition between the Kondo effect and the RKKY interaction by varying the concentration of the molecules with an unpaired spin. Another variable that can be investigated is the length over which the unpaired spin can interact with the conduction electrons. This can be done by varying the length of the molecules $l$ and therefore the distance of the unpaired spins from the surface. The final variable that can be changed is the thickness of the substrate. The thinner the substrate, the larger the fraction of conduction electrons that contribute to the Kondo effect. Therefore, the focus will be on the characterization of the 2D hybrid spin systems on gold substrate. 


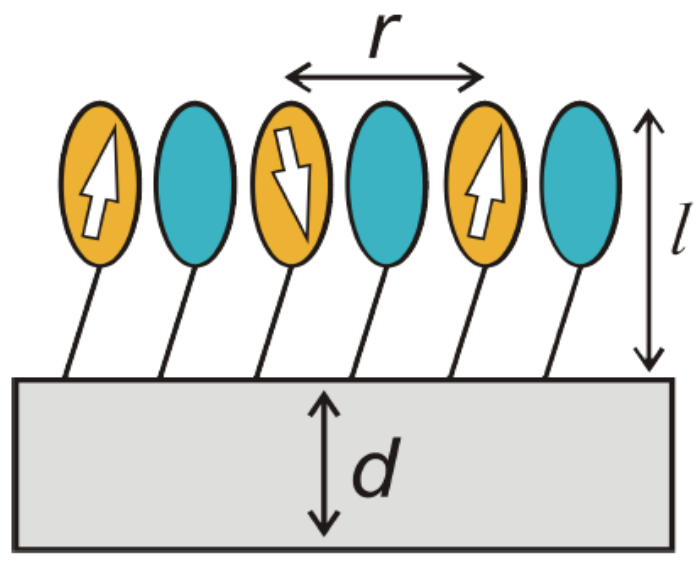

Figure 7.1 Schematic representation of a $2 \mathrm{D}$ spin system used in this project to investigate spin phenomena. It consists of a metal substrate with a monolayer of organic molecules with (orange) or without (blue) an unpaired spin. $d$ : the thickness of the substrate, $l$ : the distance between the unpaired spins and the substrate, and $r$ : the distance between the unpaired spins on the surface.

\subsection{Results and discussion}

\subsubsection{Monolayer fabrication and characterization}

Nitroxyl 2,2,6,6-tetramethylpiperidin-1-oxyl (TEMPO) radicals and terpyridinylmetal complexes $\left[\mathrm{Co}(\mathrm{Tpy}-\mathrm{SH})_{2}, \mathrm{Zn}(\mathrm{Tpy}-\mathrm{SH})_{2}, \mathrm{Co}(\mathrm{Tpy})(\mathrm{Tpy}-\mathrm{SH})\right.$, and $\left.\mathrm{Zn}(\mathrm{Tpy})(\mathrm{Tpy}-\mathrm{SH})\right]$ used in this study were synthesized according to literature procedures. ${ }^{[14]}$ As the use of thiols and disulfides for self-assembled monolayers on gold is well-known, all compounds were used for direct functionalization on gold. The monolayers were formed by simple dipping of the gold substrate in a typically $1 \mathrm{mM}$ solution of the compound in acetonitrile overnight at room temperature. The molecules studied here are depicted in Figure 7.2. Using the molecule $\mathrm{Co}(\mathrm{Tpy}-\mathrm{SH})_{2}$ Park et al. have shown the Kondo effect in the case of transport through a 
single molecule. ${ }^{[15]}$ Their experiment shows that electrons can indeed tunnel to and from the molecule in a suffcient rate to screen the magnetic impurity. We therefore expect Co(Tpy$\mathrm{SH})_{2}$ and $\mathrm{Co}(\mathrm{Tpy})(\mathrm{Tpy}-\mathrm{SH})$ complexes to be suitable to be used in the proposed Kondo system. To reduce the RKKY interaction which could interfere with the Kondo effect, $\mathrm{Zn}(\mathrm{Tpy}-\mathrm{SH})_{2}$ and $\mathrm{Zn}(\mathrm{Tpy})(\mathrm{Tpy}-\mathrm{SH})$ complexes were designed and synthesized for the diamagnetic nature of zinc atom. To lower the concentration of the Co-complexes on the Au surface, $\mathrm{Zn}$-complexes were added to the solution. By this dilution, the magnetic Co ions are placed further apart, and this changes the strength of the RKKY interaction.

Due to the existence of an unpaired spin and its inert nature, TEMPO is widely used as a probe in electron paramagnetic resonance (EPR) spectroscopy for biological systems. Since TEMPO is very stable and also carries an unpaired spin, it is very suitable for this project. For this purpose, the TEMPO-1 and TEMPO-2 adsorbates were synthesized. They only differ in the length of the linker. TEMPO-1 has a longer linker that also increases the distance between the unpaired electron and the gold film, which should decrease the coupling and the Kondo temperature.<smiles>C[N+](=O)c1ncccc1-c1cc(S)cc(-c2cc(S)cc(-c3ccccn3)n2)n1</smiles>

$\mathrm{M}(\mathrm{Tpy}-\mathrm{SH})_{2}$<smiles>c1ccc(-c2cccc(-c3ccccn3)n2)nc1</smiles>

$\mathrm{M}^{2+}$<smiles>Sc1cc(-c2ccccn2)nc(-c2ccccn2)c1</smiles>

$M(T p y)(T p y-S H)$

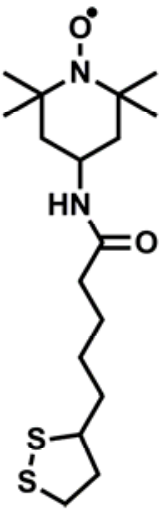

TEMPO-1<smiles>CC1(C)CC(NC(=O)CS)CC(C)(C)N1[O]</smiles>

TEMPO-2

$$
\mathrm{M}^{2+}=\mathrm{Co}^{2+} \text { or } \mathrm{Zn}^{2+}
$$

Figure 7.2 Unpaired spin adsorbate molecules and control systems used for monolayer formation onto flat gold substrates. 


\subsubsection{Characterization of terpyridinyl-metal SAMs on gold}

The monolayer formation was initially characterized by X-ray photoelectron spectroscopy (XPS) for the terpyridinyl- $\mathrm{Co}^{2+}$ and $\mathrm{Zn}^{2+}$ complexes. XPS measurements identified the cobalt ions in the monolayer to be in the $\mathrm{Co}^{2+}$ oxidation state (Figure 7.3). The two main peaks at 781.3 and $796.3 \mathrm{eV}$, corresponding to the $\mathrm{Co} 2 \mathrm{p}_{3 / 2}$ and $C 02 \mathrm{p}_{1 / 2}$ state, respectively, are representative for a system with a $\mathrm{Co}^{2+}$ ion in an octahedral structure. ${ }^{[15]}$ The satellite peaks are associated with outer-shell excitations parallel to the photoionization of an inner-shell electron, which disturbs the central potential. In the XPS spectrum, the satellite peaks of $\mathrm{Co} 2 \mathrm{p}_{3 / 2}$ and $2 \mathrm{p}_{1 / 2}$ are $787.3 \mathrm{eV}$ and $802.3 \mathrm{eV}$, respectively, which are in perfect agreement with literature values (octahedrally coordinated $\mathrm{Co}^{2+}$ ions have about $6.2 \mathrm{eV}$ satellite splittings of both Co2 $\mathrm{p}_{3 / 2}$ and Co2 $\mathrm{p}_{1 / 2}$ levels). ${ }^{[16]}$ For a full monolayer of the zinc complex, only one peak was observed at $1022 \mathrm{eV}$ as shown in Figure 7.4.

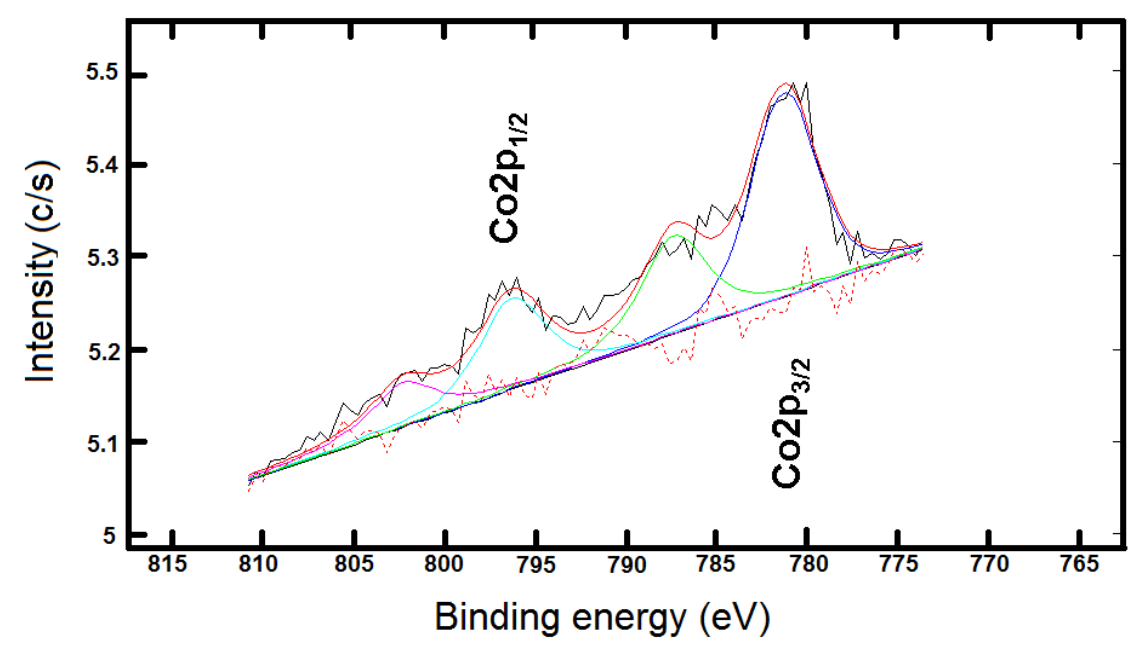

Figure 7.3 XPS spectrum (intensity vs. binding energy) of the terpyridinyl-Co complexes. The two peaks at 781.3 and $796.3 \mathrm{eV}$ correspond to the Co2 $\mathrm{p}_{3 / 2}$ and Co2 $\mathrm{p}_{1 / 2}$ states, respectively. 


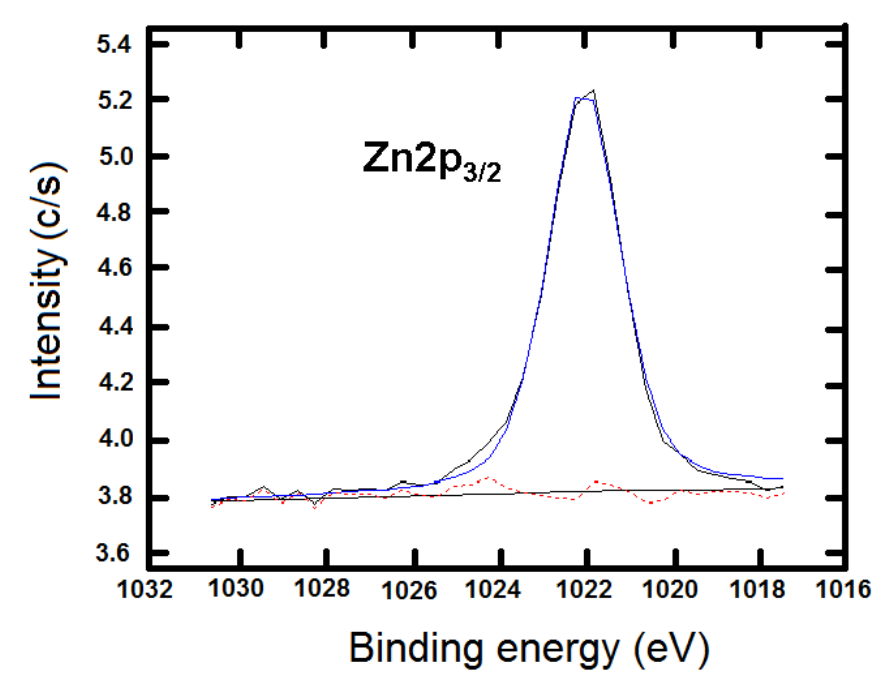

Figure 7.4 XPS spectrum (intensity in $c / s$ vs. binding energy) of the terpyridinyl-Zn complexes. The peak at $1022 \mathrm{eV}$ corresponds to the $\mathrm{Zn} 2 \mathrm{p}_{3 / 2}$ state.

To confirm the oxidation state of the $\mathrm{Co}(\mathrm{Tpy}-\mathrm{SH})_{2}$ and $\mathrm{Co}(\mathrm{Tpy})(\mathrm{Tpy}-\mathrm{SH})$ complexes, cyclic voltammetry (CV) measurements were performed. Figure 7.5 illustrates the CVs of the $\mathrm{Co}$ (Tpy)(Tpy-SH) SAM on gold in $\mathrm{CH}_{3} \mathrm{CN}$ with $0.1 \mathrm{M} \mathrm{NaClO}_{4}$ as a function of scan rate. The voltammetric waves exhibit the symmetric shape indicating a surface confined redox process. The CV showed a typical reversible electrochemical reaction, with an oxidation peak at $0.22 \mathrm{~V}$ corresponding to the $\mathrm{Co}^{3+/ 2+}$ process, which is in perfect agreement with literature values. ${ }^{[14-15]}$ The electron transfer kinetics could be determined by plotting the peak current, $I_{p}$ vs. the scan rate, $v$ (Figure 7.6). A linear correlation was obtained, with a slope of $\sim 1.0$. This indicates that the nature of the redox process is a diffusionless thus indicating a surfaceconfined process. ${ }^{[17]}$ 


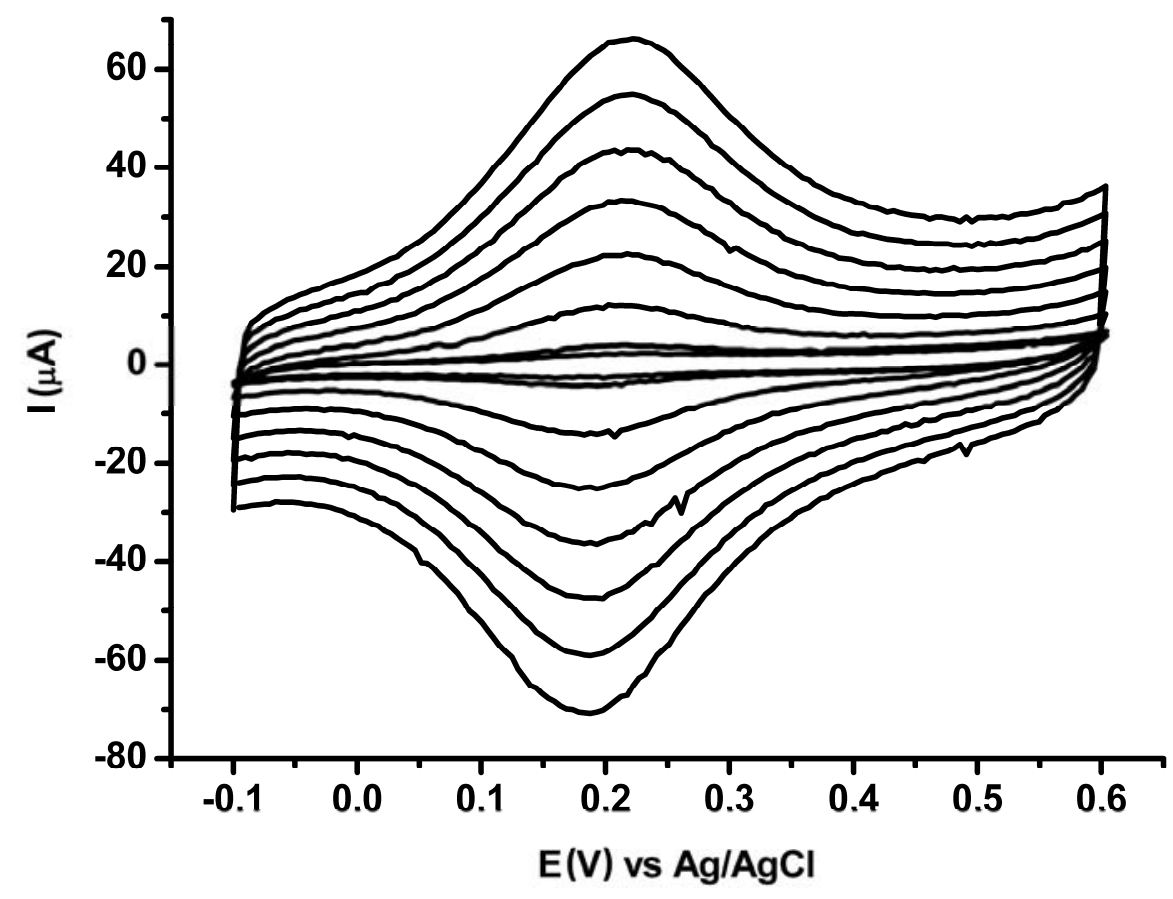

Figure 7.5 Cyclic voltammograms of a SAM of the Co(Tpy)(Tpy-SH) complex on gold in $0.1 \mathrm{M} \mathrm{NaClO}_{4} / \mathrm{CH}_{3} \mathrm{CN}$ at scan rates varying from $0.075 \mathrm{~V} / \mathrm{s}$ to $0.7 \mathrm{~V} / \mathrm{s}$.

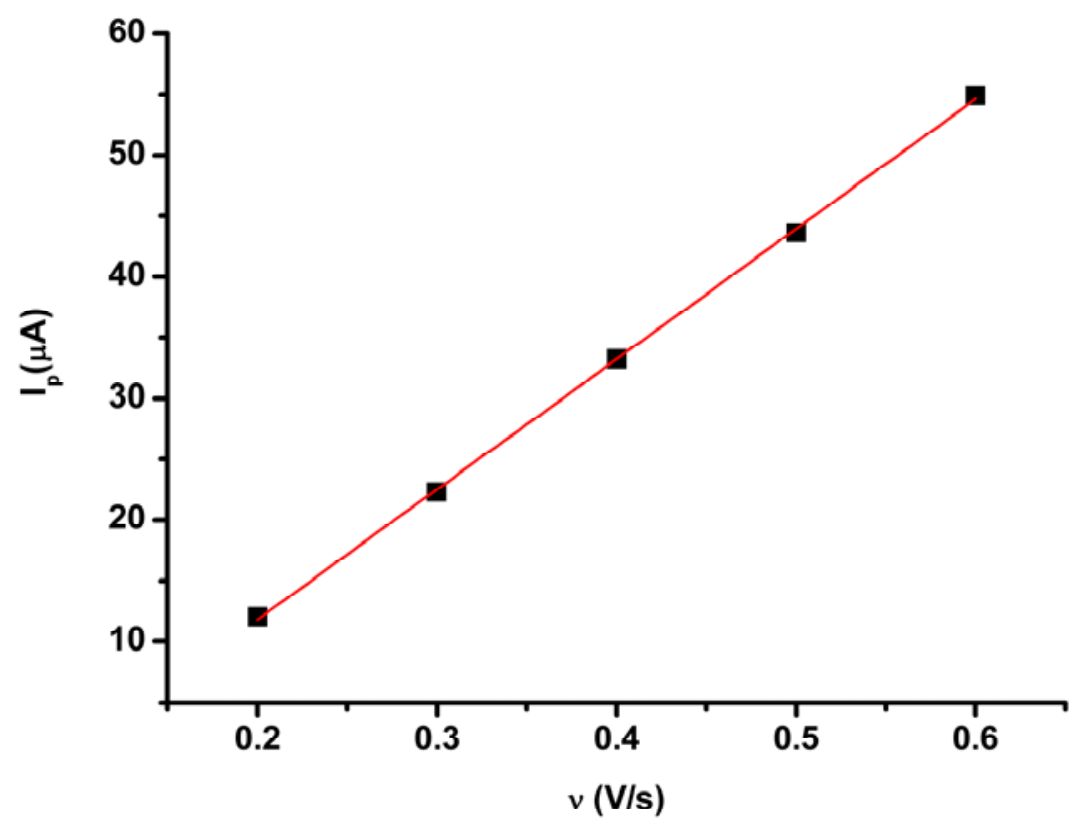

Figure 7.6 Plot of the oxidation peak current, $I_{p}$, vs. scan rate, $v$ for the $\mathrm{Co}(\mathrm{Tpy})(\mathrm{Tpy}-\mathrm{SH})$ SAM 
$\mathrm{CV}$ measurements of the $\mathrm{Co}(\mathrm{Tpy}-\mathrm{SH})_{2} \mathrm{SAM}$ are shown in Figure 7.7. Using the molecule Co(tpy-SH) 2 , Park et al. have shown the Kondo effect in the case of transport through a single molecule. ${ }^{[15]}$ Their experiment shows that electrons can indeed tunnel to and from the molecule in a suffcient rate to screen the magnetic impurity. The CV showed a typical reversible electrochemical reaction with an oxidation peak at $0.22 \mathrm{~V}$, which is similar to the Co(Tpy)(Tpy-SH) SAM. A similar electron transfer kinetics was observed for the $\mathrm{Co}(\mathrm{Tpy}-\mathrm{SH})_{2}$ (Figure 7.8). A linear correlation by plotting the peak current, $\mathrm{I}_{\mathrm{p}}$ vs. the scan rate, $v$ was obtained, with a slope of $\sim 1.0$. This confirms the surface-confined redox process.

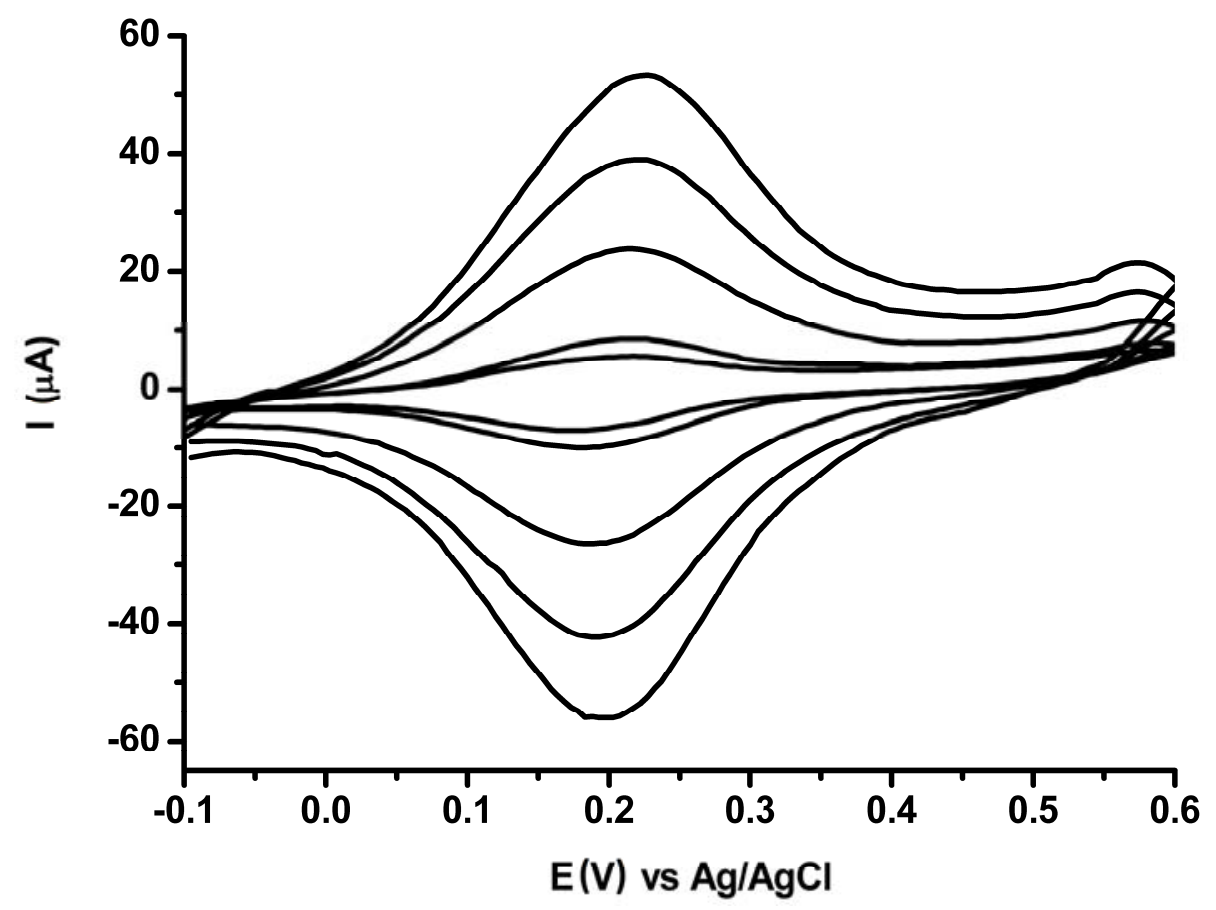

Figure 7.7 Cyclic voltammograms of a SAM of $\mathrm{Co}(\mathrm{Tpy}-\mathrm{SH})_{2}$ on gold in $0.1 \mathrm{M}$ $\mathrm{NaClO}_{4} / \mathrm{CH}_{3} \mathrm{CN}$ at scan rates varying from $0.075 \mathrm{~V} / \mathrm{s}$ to $0.4 \mathrm{~V} / \mathrm{s}$. 


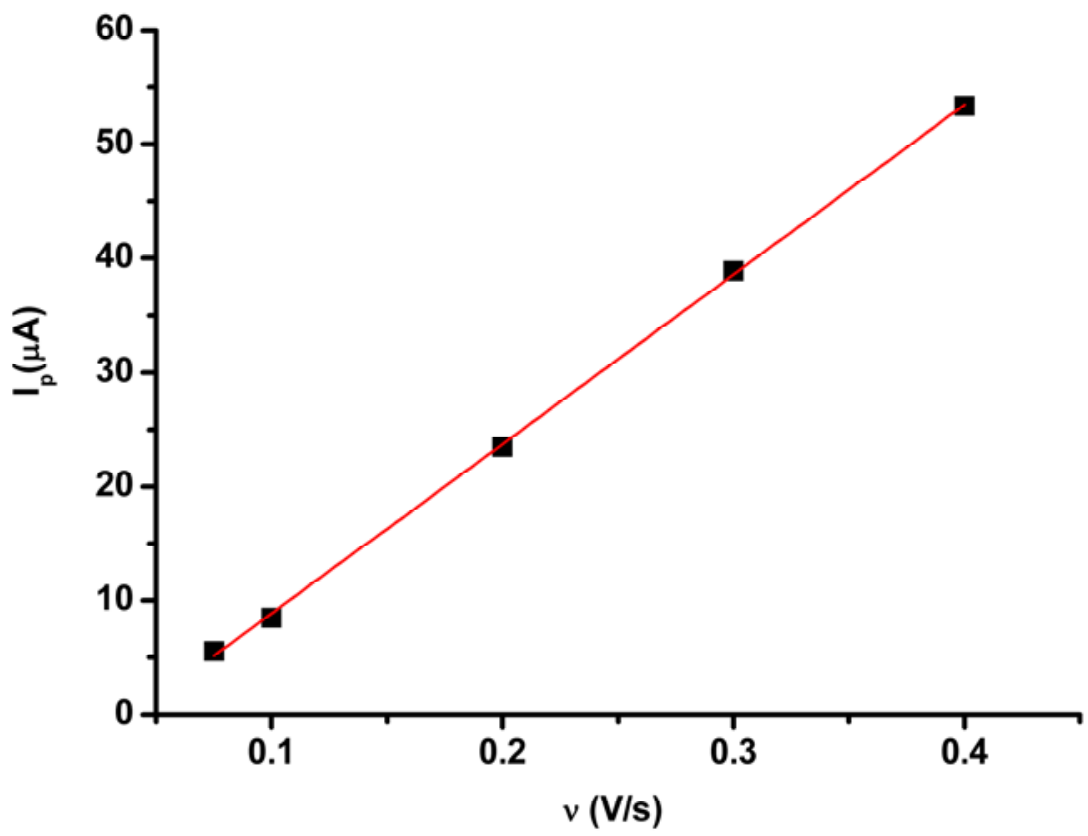

Figure 7.8 Plot of the oxidation peak current, $\mathrm{I}_{\mathrm{p}}$, vs. scan rate, $v$ for the $\mathrm{Co}(\mathrm{Tpy}-\mathrm{SH})_{2} \mathrm{SAM}$.

\subsubsection{Characterization of TEMPO SAMs on gold}

CV measurements of TEMPO SAMs exhibited a reversible one-electron process in $\mathrm{CH}_{3} \mathrm{CN}$ (vs $\mathrm{Ag} / \mathrm{AgCl}$ in $0.1 \mathrm{M} \mathrm{NaClO}_{4}$ ). Figure 7.9 shows the $\mathrm{CV}$ of the TEMPO-2 molecule on a gold electrode. The redox potentials were $+0.72 \mathrm{~V}$ and $+0.68 \mathrm{~V}$ vs $\mathrm{Ag} / \mathrm{AgCl}$, respectively, which is in good agreement with literature values. ${ }^{[18]}$ The peak split between the anodic and cathodic peak potentials was about $40 \mathrm{mV}$ and a linear relationship was observed between the peak current and potential scan rate ranging from $0.050 \mathrm{~V} / \mathrm{s}$ to $0.2 \mathrm{~V} / \mathrm{s}$. It is important to note that the monolayer was quite stable and no decay of current was observed after 20 repetitive cycles at $0.2 \mathrm{~V} / \mathrm{s}$ on gold. 


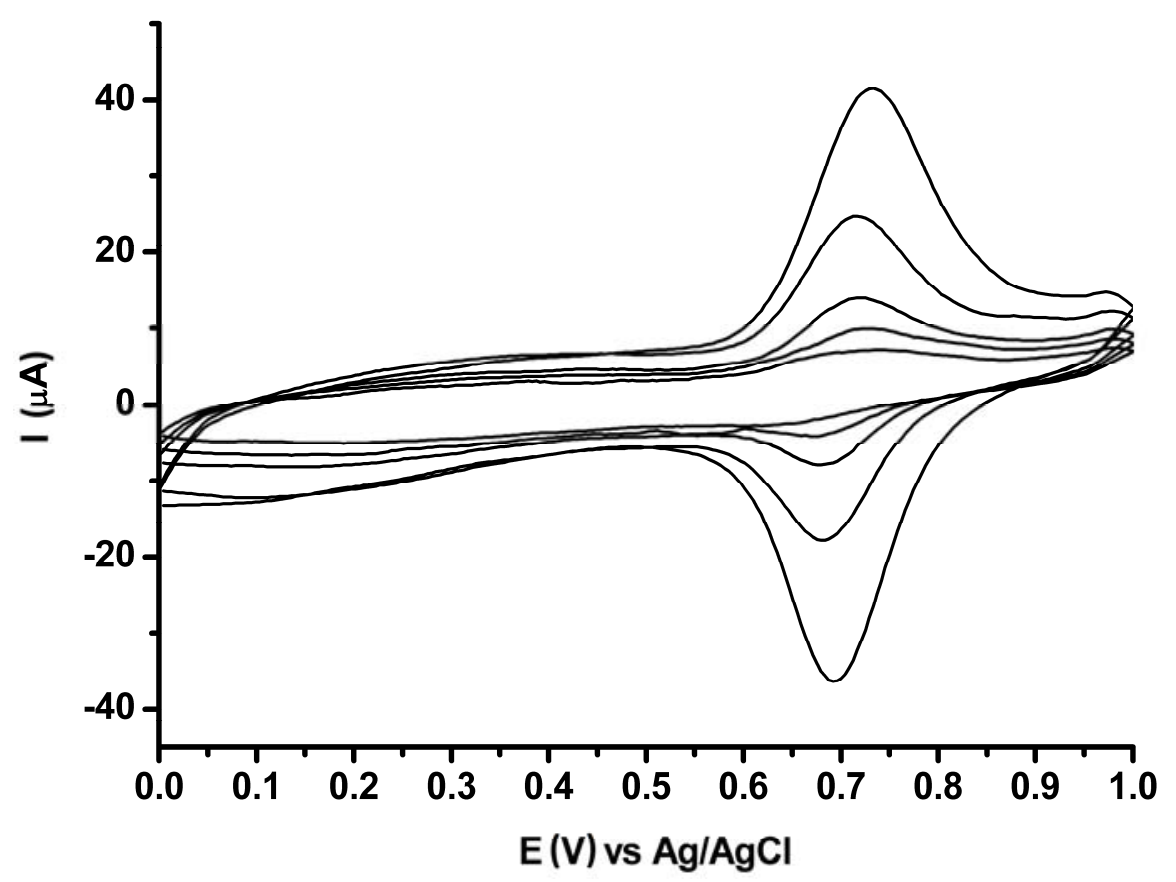

Figure 7.9 Cyclic voltammograms of a SAM of the TEMPO-2 on gold in $0.1 \mathrm{M}$ $\mathrm{NaClO}_{4} / \mathrm{CH}_{3} \mathrm{CN}$ at scan rates varying from $0.050 \mathrm{~V} / \mathrm{s}$ to $0.2 \mathrm{~V} / \mathrm{s}$.

CVs of the TEMPO-1 SAM on gold is shown in Figure 7.10. The CV exhibited a symmetrical reversible one electron redox process. The redox potentials were $+0.70 \mathrm{~V}$ and $+0.68 \mathrm{~V}$ vs $\mathrm{Ag} / \mathrm{AgCl}$, respectively. Unfortunately, the pure monolayer was somewhat unstable during electrochemical measurements and a continuous decrease of current was observed with repetitive cycles at different scan rates. ${ }^{[19]}$ 


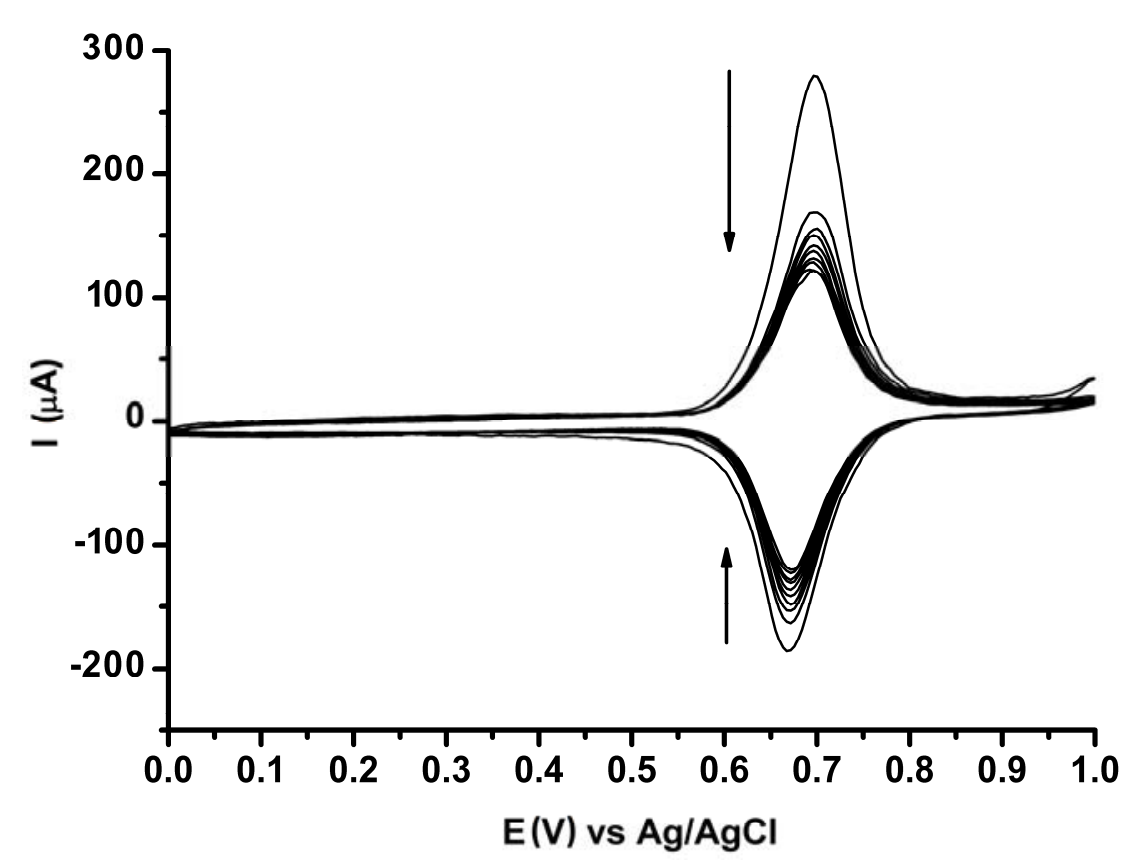

Figure 7.10 Cyclic voltammograms of a SAM of TEMPO-1 on gold in $0.1 \mathrm{M}$ $\mathrm{NaClO}_{4} / \mathrm{CH}_{3} \mathrm{CN}$ at $0.2 \mathrm{~V} / \mathrm{s}$ for 10 repetitive cycles.

For the further characterization of radical SAMs, electron paramagnetic resonance (EPR) measurements were performed on gold substrates functionalized with nitroxide radicals by Veronica Mugnaini at the Institute of Material Sciences of the University Autonoma, Barcelona. An EPR spectrum of the TEMPO-2 SAM is shown in Figure 7.11. The only signal was observed at $\sim 3455 \mathrm{G}$, indicating the existence of a single spin on gold surface. 


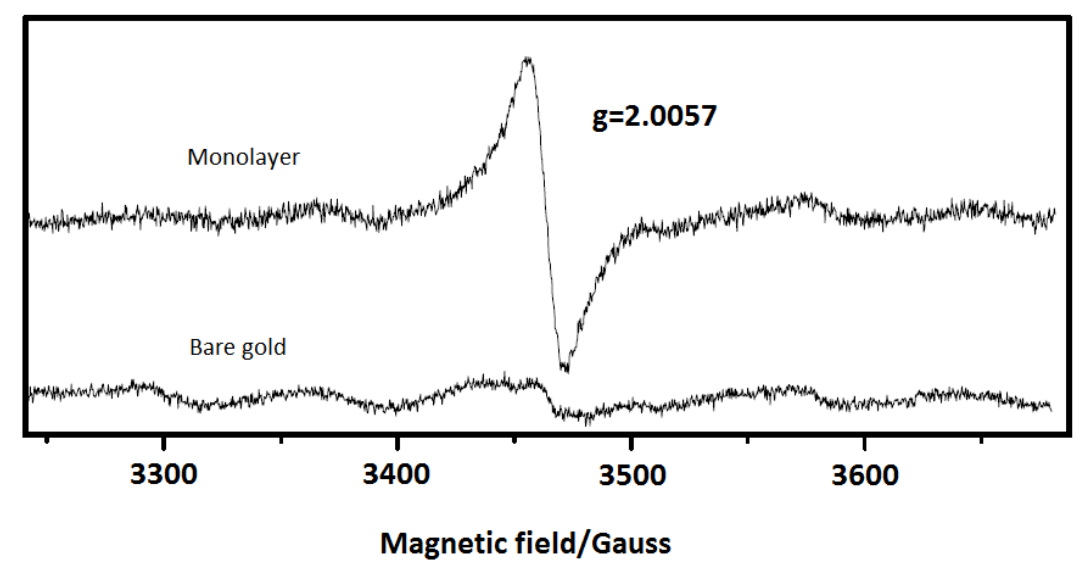

Figure 7.11 EPR spectra of a SAM of TEMPO-2 on gold (top spectrum) and of a bare gold substrate (bottom).

\subsubsection{Electrical characterization}

This part of the chapter describes initial resistance measurements of gold thin films covered with monolayers of terpyridinyl-metal complexes and TEMPO molecules. Measurements were carried out with the cryogenic measurement equipment by Wouter Naber for the metal complexes ${ }^{[20]}$ and Peter Tijssen for the TEMPO molecules ${ }^{[21]}$ in the Nanoelectronics group of the University of Twente.

The Van der Pauw measurement technique was used to determine the sheet resistance $R_{s}$ of gold samples. After self-assembly of the monolayer, the substrate is wirebonded at the four corners and measured with a Physical Properties Measurements System (PMMS) of Quantum Design. ${ }^{[20]}$ With these four contacts, Hall measurements can also be performed, to determine the charge carrier density in samples to see if this is influenced by the SAM fomation. Hall measurements in all samples gave a carrier density in the Au of $\sim 5.2$ $\mathrm{x} 10^{28} \mathrm{~m}^{3}$, close to the value of bulk $\mathrm{Au}\left(\sim 5.9 \times 10^{28} \mathrm{~m}^{3}\right)$, showing the quality of Au layers and the fact that the Au is unaffected by the self-assembly process. 


\subsubsection{Characterization of Co(Tpy-SH $)_{2}$ SAMs on gold}

The measurements described in this section have been performed on a $\mathrm{Si} / \mathrm{SiO}_{2}-(\sim 1$ $\mathrm{nm}$ ) substrates covered with a $10 \mathrm{~nm}$ thick $\mathrm{Au}$ layer. The Au layers were cleaned with acetone and 2-propanol, and then with piranha solution before SAM formation. The Co(Tpy$\mathrm{SH})_{2}$ complex was the same as used by Park et al., with a thiol group on both sides of the Cocomplex. ${ }^{[15]}$ A drawback of the $\mathrm{Co}(\mathrm{Tpy}-\mathrm{SH})_{2}$ is the possible formation of polymers by the coupling of two thiol groups. Different samples with different ratios of $\mathrm{Co}(\mathrm{Tpy}-\mathrm{SH})_{2}$ and $\mathrm{Zn}(\mathrm{Tpy}-\mathrm{SH})_{2}$ were measured. Although it was not expected that this would give absolute control over the concentration of the Co complexes in the monolayer, dilution with the $\mathrm{Zn}$ complex is at least expected to result in a lower concentration of Co complex on the gold surface and hence to a lower interaction between the $\mathrm{Co}(\mathrm{Tpy}-\mathrm{SH})_{2}$ complexes (e.g. a reduced RKKY interaction). We fabricated samples with only Co-complex, only Zn-complex, and both Co- complex and Zn-complex in the ratios 1:1, 1:100 and 1:1000.

Temperature-dependent measurements of the sheet resistance $R_{s}$ of $10 \mathrm{~nm}$ Au layers covered with SAMs are shown in Figure 7.12. The samples with only Zn(Tpy-SH) ${ }_{2}$ show a saturation of the resistance around $10 \mathrm{~K}$ (Figure $7.12 \mathrm{a}$ ). Below this temperature, $\mathrm{R}_{\mathrm{s}}$ stays constant as expected for a metal without any magnetic impurities. The same behavior is observed for the samples with only $\mathrm{Co}(\mathrm{Tpy}-\mathrm{SH})_{2}$ (Figure $7.12 \mathrm{~b}$ ), and for the ratio 1:1000 (Figure 7.12d). A small increase in the resistance was observed when the temperature was decreased below $10 \mathrm{~K}$. A large increase in the resistance when the temperature is lowered was seen in some of the samples with the ratio 1:100 (Figure 7.12f, filled squares). This increase is as high as several percent as compared to the minimum value at $\sim 20 \mathrm{~K}$. This behavior is however not always observed in similar samples, as is shown in Figure 7.12e. The temperature-dependent measurements in the presence of a magnetic field of $8 \mathrm{~T}$ have performed. The measurement at $\mathrm{H}=8 \mathrm{~T}$ is shown in Figure $7.12 \mathrm{f}$ (open circles). Almost no 
change was seen as compared to the measurement at $\mathrm{H}=0 \mathrm{~T}$ (Figure 7.12f, filled squares). The temperature behavior only showed a very small upturn in the resistance when the temperature was lowered, despite the fact that the samples were fabricated in the same way and the $\mathrm{Co} / \mathrm{Zn}$ ratio was the same. The difference may originate from formation of a disordered SAM. Another option might be the formation of polymers by the Co- and Zncomplexes, because of the two functional SH groups.

(a)

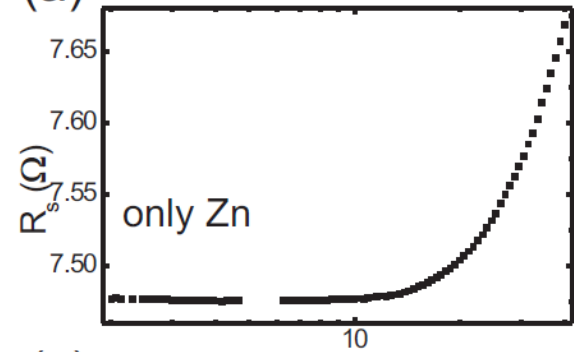

(c)

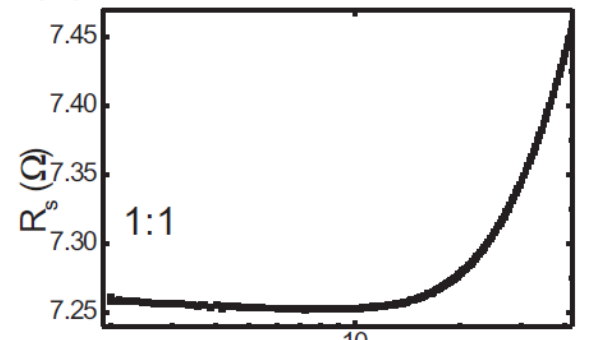

(e)

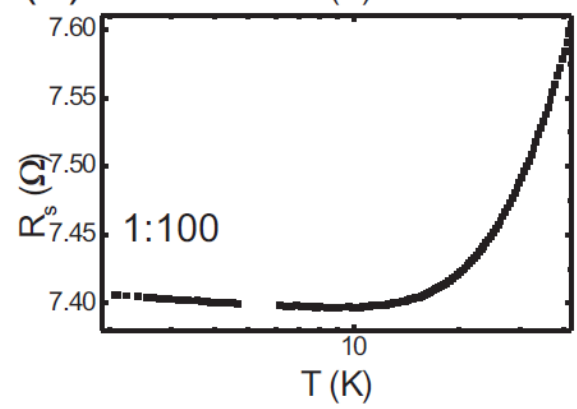

(b)

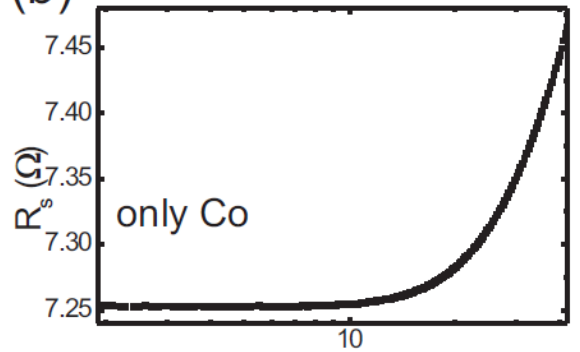

(d)

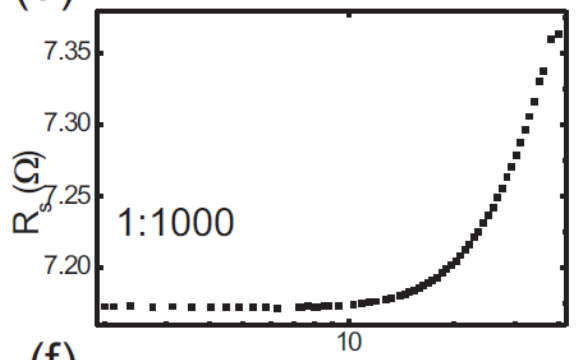

(f)

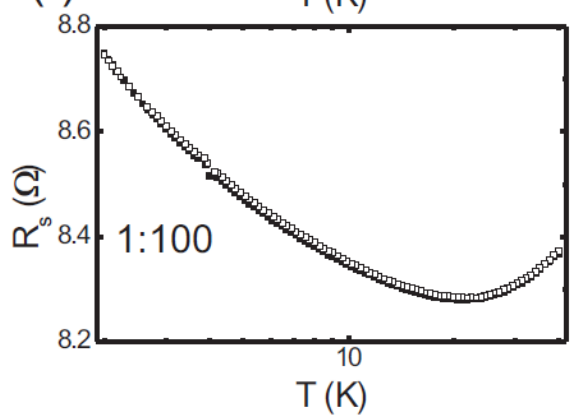

Figure 7.12 Sheet resistance $\mathrm{R}_{\mathrm{s}}$ vs. temperature $\mathrm{T}$ for samples with a $10 \mathrm{~nm}$ Au layer covered with a SAM consisting of (a) $\mathrm{Zn}(\mathrm{Tpy}-\mathrm{SH})_{2}$, (b) $\mathrm{Co}(\mathrm{Tpy}-\mathrm{SH})_{2}$, and Co- and $\mathrm{Zn}$-complexes in the ratios (c) 1:1, (d) 1:1000, (e) 1:100, showing a small resistance increase and (f) 1:100, showing a large resistance increase without (filled squares) and with (open circles) a magnetic field. 


\subsubsection{Characterization of Co(Tpy)(Tpy-SH) SAM on gold}

To exclude impurities and polymer formation in samples, the Co(Tpy)(Tpy-SH) and $\mathrm{Zn}(\mathrm{Tpy})(\mathrm{Tpy}-\mathrm{SH})$ which contain only one thiol group was synthesized. Dilutions were done similarly. We fabricated samples with only Co-complex, only Zn-complex, and both Co- and Zn-complexes in the ratios 1:1000, 1:5000 and 1:10000. The given ratios are the ratios as used in the solutions prior to SAM formation.

Temperature-dependent measurements of the sheet resistance $R_{s}$ of $10 \mathrm{~nm}$ Au layers covered with SAMs are shown in Figure 7.13. It clearly shows that in case of only Cocomplex, Zn-complex, and Co:Zn ratio of 1:10000, the resistance saturated with decreasing temperature below $10 \mathrm{~K}$, which was expected because of the lack of paramagnetic molecules for only $\mathrm{Zn}$, strong RKKY interaction between spins for only Co-complex, and very low concentration of Co-complex for 1:10000 diluted sample. However, the samples with Co:Zn ratios of 1:1000 and 1:5000 showed a relative resistance rise of several percent (compared to the minimum sheet resistance), for approximately $40 \%$ of the measured samples. One of the samples with a ratio of 1:5000 was measured at lower temperatures, down to $\sim 0.27 \mathrm{~K}$, as shown in Figure 7.14. Temperature is plotted here on a logarithmic scale, which clearly shows the logarithmic increase between 0.5 and $5 \mathrm{~K}$. This is another strong indication that Kondo physics is occurring in these samples, but th effect remains in the presence of a large magnetic field, which is not expected for the Kondo effect. A big problem of the measured samples is the irreproducibility of the observed results, indicating the lack of control over the self-assembly, sample preparation, and/or measurement process. More experiments are needed to get a clearer picture of the behavior of the observed effect. 
(a)

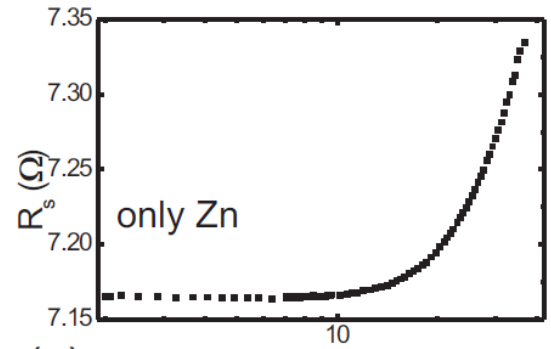

(c)

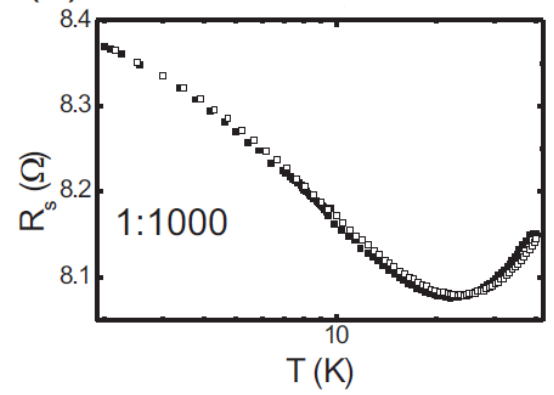

(b)

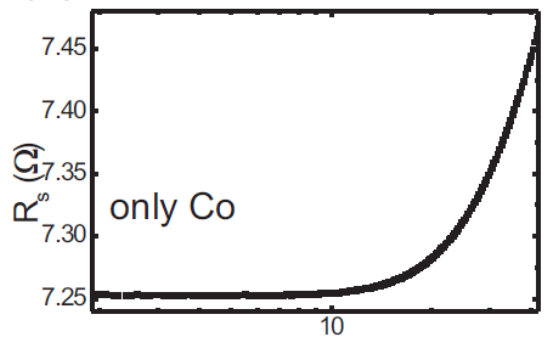

(d)

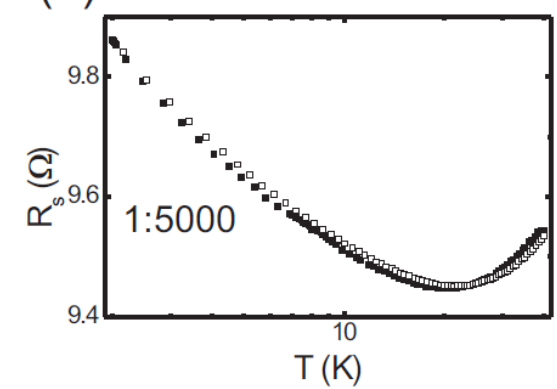

Figure 7.13 Sheet resistance $R_{s}$ vs. temperature $T$ for samples with a $10 \mathrm{~nm}$ Au layer covered with a SAM consisting of (a) only Zn(Tpy)(Tpy-SH), (b) only Co(Tpy)(Tpy-SH) and Co- and Zn-complexes in the ratio (c) 1:1000, (d) 1:5000, showing a resistance increase, without (filled squares) and with (open circles) a magnetic field.

(a)

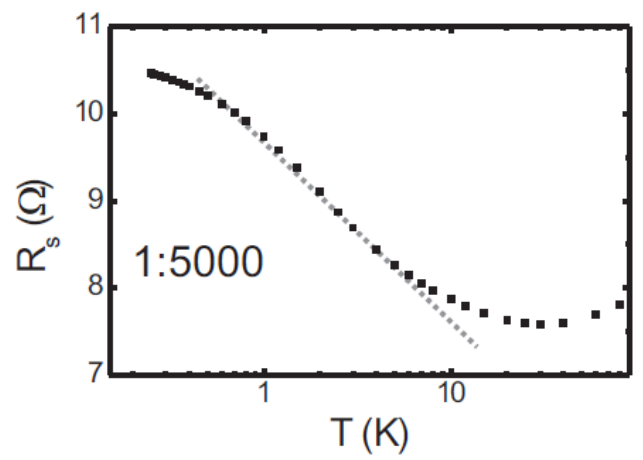

(b)

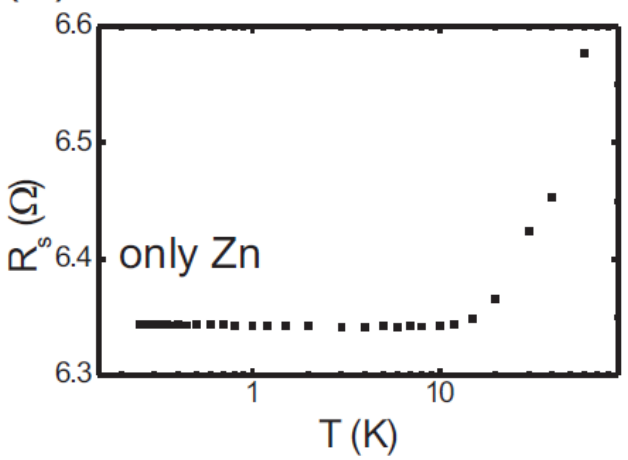

Figure 7.14 Sheet resistance $R_{s}$ vs. temperature $T$ of a $10 \mathrm{~nm}$ Au layer covered with a SAM of (a) Co- and Zn-complexes in the ratio 1:5000 and (b) only Zn complexes. 


\subsubsection{Characterization of TEMPO SAMs on gold}

This section describes the results of the resistance measurements on gold thin films covered with monolayers of TEMPO molecules. The first measurements on the $10 \mathrm{~nm}$ thick gold films, covered with a full (i.e. without dilution) monolayer of the TEMPO-1 were carried out and no significant difference in resistance between the bare $10 \mathrm{~nm}$ gold and the gold covered with the monolayer was observed which is shown in Figure 7.15. Here the blue line indicates the resistance of the $10 \mathrm{~nm}$ bare gold film and the red line indicates the resistance of the gold sample covered with a monolayer. Both curves showed a resistance rise which is logarithmically dependent on temperature. It can be concluded that this resistance rise is caused by magnetic impurities, and that the TEMPO-1 SAM does not show Kondo behaviour.

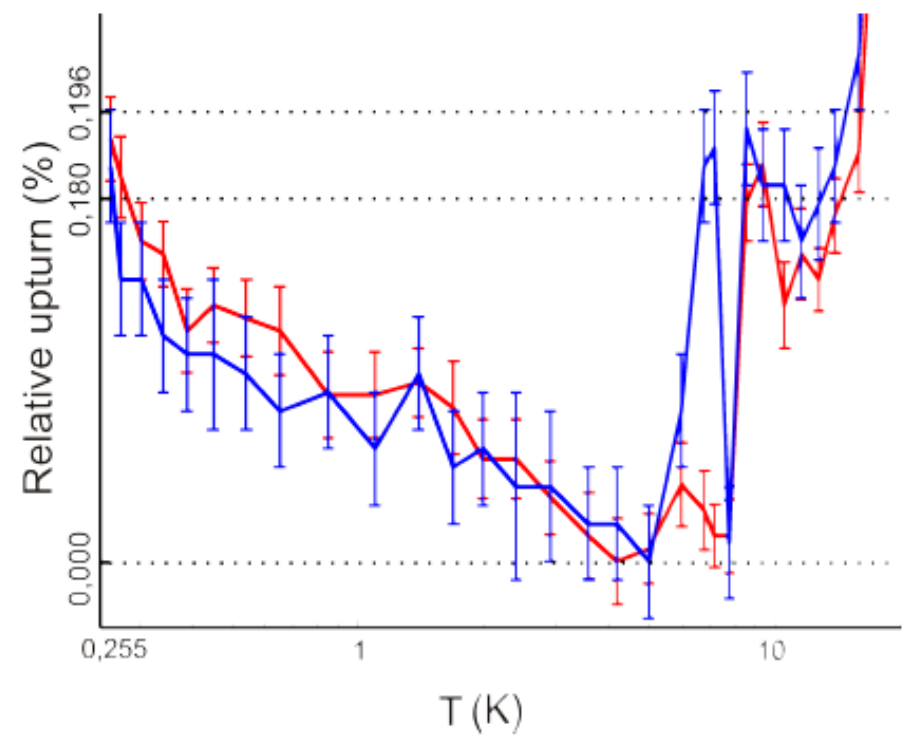

Figure 7.15 Relative resistance of two $10 \mathrm{~nm}$ thick square gold films (4 by $4 \mathrm{~mm}$ ) versus temperature. One bare gold film (blue line) and one covered with a monolayer made of the TEMPO-1 (red line). 
As already shown by Park et al. for the Co complexes, decreasing the distance between the unpaired spin and the conductive substrate will result in a better coupling between the states of the magnetic molecule and the electrodes. ${ }^{[15]}$ This is because the tunneling probability increases exponentially with decreasing tunnel barrier thickness. ${ }^{[22]}$ Therefore this was also expected for the monolayer made of the TEMPO-2. Monolayers of the TEMPO-2 were fabricated on both the 5 and $10 \mathrm{~nm}$ thick gold films. For the $5 \mathrm{~nm}$ thick film a typical result is shown in Figure 7.16. This graph clearly indicates that the curves of both the bare gold film and the one covered with the TEMPO-2 have the same shape. For the $10 \mathrm{~nm}$ thick films the same behaviour was observed. It can be concluded that the expected significant difference in relative resistance rise between systems consisting of bare gold thin films and systems consisting of these gold thin films covered with TEMPO-1 and TEMPO-2 was not observed. It could be expected from the $\mathrm{CV}$ measurements that the unpaired electrons from Cocomplexes can more easily tunnel to the gold substrate than the TEMPO radicals, because of the peak potential which was approx. $0.5 \mathrm{~V}$ lower for the Co-complex and both measurements were obtained with a $\mathrm{Ag} / \mathrm{AgCl}$ reference electrode. This could therefore lead to a less pronounced Kondo effect for the TEMPO derivatives.

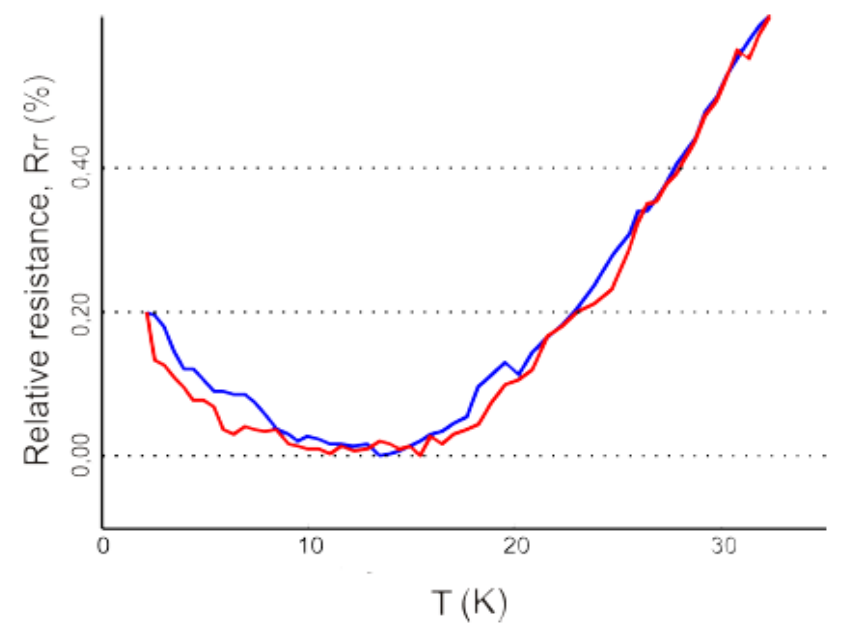

Figure 7.16 Relative resistance of two $5 \mathrm{~nm}$ thick square gold films (4 by $4 \mathrm{~mm}$ ) versus temperature. One bare gold film (blue) and one covered with the TEMPO-2 (red). 


\subsection{Conclusions}

In conclusion, we have developed two-dimensional organic spin systems to investigate possible Kondo effets on gold surfaces. To this purpose, nitroxyl 2,2,6,6tetramethylpiperidin-1-oxyl (TEMPO) radicals and terpyridinyl-metal complexes [Co(Tpy$\mathrm{SH})_{2}, \mathrm{Zn}(\mathrm{Tpy}-\mathrm{SH})_{2}, \mathrm{Co}(\mathrm{Tpy})(\mathrm{Tpy}-\mathrm{SH})$, and $\left.\mathrm{Zn}(\mathrm{Tpy})(\mathrm{Tpy}-\mathrm{SH})\right]$ were synthesized. The formation of SAMs on gold was characterized by XPS and CV measurements. The electrical measurements showed an increase in the sheet resistance when the temperature was decreased for $10 \mathrm{~nm}$ Au layers covered with a mix SAM of terpyridinyl-metal complexes (magnetic and non-magnetic molecules). This effect was only observed for specific ratios of the two different molecules. This effect has been reproduced in different samples and different measurement setups. At the same time, the resistance increase was not always observed in identically prepared samples. However, it was never observed for samples without any magnetic ions, making it very likely to be a magnetic effect. The observed effect might indicate the presence of Kondo screening, but the effect remains in the presence of a large magnetic field, which is not expected for the Kondo effect. Hence, more efforts are needed to widely understand observed effects as mentioned in this chapter.

\subsection{Acknowledgements}

The work presented in this chapter was performed in collaboration with the Nanoelectronics group at the University of Twente. Prof. Dr. Wilfred van der Wiel conceived the project. Dr. Wouter Naber and Peter Tijssen carried out the electrical measurements. Aldrik Velders and Michael de Jong are acknowledged for fruitful discussions and help. Gerard Kip is acknowledged for XPS measurements. 


\subsection{Experimental section}

Substrate and monolayer preparation. $\mathrm{Si} / \mathrm{SiO}_{2}-(\sim 1 \mathrm{~nm})$ substrates covered with a $10 \mathrm{~nm}$ thick Au layer were provided by the Nanoelectronics group. The substrates were cleaned with piranha solution (concentrated $\mathrm{H}_{2} \mathrm{SO}_{4}$ and $33 \%$ aqueous $\mathrm{H}_{2} \mathrm{O}_{2}$ in a 3:1 ratio; Caution: piranha should be handled carefully) for $15 \mathrm{~s}$ and rinsed with MilliQ water and EtOH . After drying in a nitrogen stream, the substrates were used immediately for monolayer formation. The monolayers were formed by simple dipping of the gold substrate in a typically $1 \mathrm{mM}$ of compounds in acetonitrile solution overnight at room temperature. After overnight incubation, the slides were rinsed with acetonitrile to remove any excess of compounds and subsequently dried in a nitrogen stream.

X-ray photoelectron spectroscopy (XPS). XPS was performed on a PHI Quantera SXM, using a monochromated Al K alfa X-ray source with an energy of $1486.6 \mathrm{eV}$. An X-ray beam with a diameter of 100 micrometer and a power of $25 \mathrm{~W}$ was used.

Electrochemistry. Electrochemical measurements were performed with an AUTOLAB PGSTAT10 in a custom built three-electrode setup with bare gold substrates as the working electrode, a $\mathrm{Ag} / \mathrm{AgCl}$ reference electrode, and a platinum wire as counter electrode. 


\subsection{References}

[1] W.J. de Haas, J.H. de Boer,G.J. van den Berg, Physica 1934, 1, 1115-1124.

[2] J. Kondo, Prog. Theor. Phys. 1964, 32, 37-49.

[3] A.C. Hewson, The Kondo problem to Heavy Fermions, Cambridge University Press, Cambridge, 1997.

[4] L.P. Kouwenhoven, L.I. Glazman, Physics World 2001, 14, 33-38.

[5] D. Goldhaber-Gordon, H. Shtrikman, D. Mahalu, D. Abusch-Magder, U. Meirav, M.A. Kastner, Nature 1998, 391, 156-159.

[6] S. Sasaki, S. De Franceschi, J.M. Elzerman, W.G. van der Wiel, M. Eto, S. Tarucha, L.P. Kouwenhoven, Nature 2000, 405, 764-767.

[7] W.G. van der Wiel, S. De Franceschi, T. Fujisawa, J.M. Elzerman, S. Taruch, L.P. Kouwenhoven, Science 2000, 289, 2105-2108.

[8] I. Affleck, P. Simon, Phys. Rev. Lett. 2001, 86, 2854-2857.

[9] G. Bergmann, Phys. Rev. B 2008, 77, 104401.

[10] M. Sarachik, E. Corenzwit, L. D. longinotti, Phys. Rev. 1964, 135, 1-3.

[11] J.J. Prejean, E. Lhotel, A. Sulpice, F. Hippert, Phys. Rev. B 2006, 73, 214205.

[12] K. Yosida, Phys. Rev. 1957, 106, 893-898.

[13] M.A. Ruderman, C. Kittel, Phys. Rev. 1954, 96, 99.

[14] a) M. Maskus, H. D. Abruna, Langmuir, 1996, 12, 4455-4462; b) V. Chechik, H. J. Wellsted, A. Korte, B. C. Gilbert, H. Caldararu, P. Ionita, A. Caragheorgheopol, Faraday Discuss. 2004, 125, 279-291.

[15] J. Park, A. N. Pasupathy, J. I. Goldsmith, C. Chang, Y. Yaish, J. R. Petta, M. Rinkoski, J. P. Sethna, H. D. Abruna, P. L. McEuen, D. C. Ralph, Nature 2002, 417, $722-725$. 
[16] M. Oku, K. Hirokawa, J. Electron. Spectrosc. 1976, 8, 475-481.

[17] D. V. Jr. Gosser, Cyclic Voltammetry: Simulation and analysis of reaction mechanisms, VCH, New York, 1993.

[18] a) O. Aleveque, F. Seladji, C. Gautier, M. Dias, T. Breton, E. Levillain, ChemPhysChem 2009, 10, 2401-2404; b) O. Aleveque, P. Y. Blanchard, T. Breton, M. Dias, C. Gautier, E. Levillain, F. Seladji, Electrochem. Commun. 2009, 11, 17761780.

[19] Y. Kashiwagi, K. Uchiyama, F. Kurashima, J. Anzai, T. Osa, Anal. Sci. 1999, 15, 907-909.

[20] W.J.M. Naber, Electron transport and spin phenomena in hybrid organic/inorganic systems, PhD thesis, University of Twente, 2010.

[21] P. Tijssen, Interaction of TEMPO-derivative $2 D$ spin systems with conduction electrons in thin gold films, MSc thesis, University of Twente, 2010.

[22] J. M. D. Coey, Magnetism and Magnetic Materials, Cambridge University Press, 2010. 


\section{Summary}

The research described in this thesis is focused on the combination of orthogonal supramolecular interactions for functional monolayer architectures on surfaces. The term "orthogonal supramolecular interactions" refers to non-covalent interactions that do not influence each other's assembly properties when applied in the same system. Orthogonal selfassembly allows extended control over the self-assembly process and promotes new materials properties. Individual noncovalent interactions (e.g. hydrogen bonding, metal coordination, electrostatic or host-guest interactions) have been employed in many studies. However, the combination of different supramolecular interactions in the same system can improve the properties of the materials. The research described in this thesis aims to develop hybrid, multifunctional monolayers by using orthogonal supramolecular interactions, enabling the control over the monolayer composition and functionality. Orthogonal host-guest and lanthanide-ligand coordination interaction motifs have been employed to create supramolecular luminescent monolayers in the first part of the thesis (Chapters 3 to 5). The second part of the thesis (Chapters 6 and 7) deals with the fabrication of functional monolayers on silicon and gold substrates for applications in electronics.

A general introduction has been given in Chapter 1 which addresses the importance of non-covalent interactions for the fabrication of functional surfaces.

A literature overview has been given in Chapter 2 on the use of supramolecular interactions for the generation of hybrid assemblies and materials. Supramolecular chemistry and molecular self-assembly including hydrogen bonding, metal coordination, electrostatic and host-guest interactions to direct the immobilization of functional systems on surfaces are 
discussed in detail. Special attention is given to the combination of different supramolecular interaction types for the generation of functional monolayer architectures.

Chapter 3 describes the assembly of a guest-functionalized naphthalene antenna and a $\mathrm{Eu}^{3+}$-complexed EDTA-based ligand molecule onto a receptor surface by combining the orthogonal host-guest and metal-ligand coordination motifs. Local fluorescence emission spectra of the immobilized complex demonstrated the occurrence of sensitized $\mathrm{Eu}^{3+}$ luminescence. The energy transfer efficiency between the antenna and the $\mathrm{Eu}^{3+}$ complex was determined by time-resolved fluorescence measurements and found to be about $35 \%$. The stoichiometry of complexation between antenna and $\mathrm{Eu}^{3+}$ complex was investigated by the very first Job plot analysis at a surface and found to be 1:1 by examining the sensitized luminescence of the surface-immobilized complex at different antenna-Eu ${ }^{3+}$ complex ratios.

In Chapter 4, the same lanthanide complex system was used for the ratiometric detection of dipicolinic acid (DPA), which is a unique biomarker for anthrax bacterial spores, with high sensitivity and selectivity on a supramolecular monolayer surface. The fluorescence intensity ratio of the lanthanide-based surface receptor system upon addition of different concentrations of DPA showed that the recovery of the naphthalene emission is practically complete in the presence of only $200 \mathrm{nM}$ DPA. The intensity ratio of the $\mathrm{Eu}^{3+}$-based sensing platform as a function of time upon addition of $200 \mathrm{nM}$ DPA indicated that the sensing response is complete within $10 \mathrm{~min}$. The detection limit of the system was also investigated and found to be about $25 \mathrm{nM}$ of DPA. To exhibit the selectivity of the supramolecular sensing surface, fluorescence changes upon addition of different competitive aromatic ligands were investigated. The negligible changes demonstrated the excellent selectivity of the system for DPA.

Chapter 5 has introduced a supramolecular sensing platform on a microchip surface that allows the detection of biologically relevant phosphate anions and aromatic carboxylic 
acids in a high-throughput format. The $\mathrm{Eu}^{3+}$-based supramolecular sensing system was implemented on the surface of a multichannel chip. The sensing layer on the microchip surface is an ensemble of the antenna and $\mathrm{Eu}^{3+}$-complexed ligand. When an anionic guest was added to the ensemble, it displaced the antenna, and triggered a fluorescence decrease upon binding to the $\mathrm{Eu}^{3+}$ center. ATP and pyrophosphate among various phosphate anions, and the anthrax biomarker DPA among various aromatic carboxylic acids showed a strong response to the sensitized $\mathrm{Eu}^{3+}$ luminescence-based microchip surface.

Chapter 6 describes the local doping of oxide-free silicon using nanoimprint lithography (NIL) and molecular monolayers. Covalently bonded Si-C monolayer patterns with feature sizes ranging from $100 \mathrm{~nm}$ to $100 \mu \mathrm{m}$ were created via a local hydrosilylation reaction on NIL-patterned resist areas. These patterns were characterized by XPS, AFM, and TOF-SIMS measurements. Monolayer patterns with $100-\mu \mathrm{m}$ features were fabricated to enable pattern characterization by XPS. Elemental mapping spectra showed that the patterned monolayers were created by hydrosilylation. To fabricate the nanoscale features, a nickelplated mold containing a square array of $150 \mathrm{~nm}$ pillars was used and the resulting structures were visualized by AFM. Results showed that clearly defined patterns were obtained. Using a phosphorus-containing organic precursor, highly-doped and patterned ( $\mu \mathrm{m}$ scale) regions in nearly intrinsic silicon were successfully fabricated. These patterned regions were characterized by TOF-SIMS for imaging and depth profiling. The dopant surface dose on a doped area measured $(2.3 \pm 0.1) \times 10^{19} \mathrm{P}$ atoms. $\mathrm{cm}^{-3}$ corresponding to an areal dose on a doped area of $5.6 \pm 0.1 \times 10^{13} \mathrm{P}$ atoms. $\mathrm{cm}^{-2}$. Electrical characterizations were performed by Hall and sheet-resistance measurements. An average sheet carrier density was determined and found to be $1.9 \pm 0.1 \times 10^{13} \mathrm{~cm}^{-2}$ at $300 \mathrm{~K}$ and $9.4 \pm 0.1 \times 10^{12} \mathrm{~cm}^{-2}$ at $150 \mathrm{~K}$ in case of patterned samples. More importantly, the resistance measured on line-patterned substrates along the 
line direction was significantly lower than when measured perpendicular to the pattern direction.

Chapter 7 describes the fabrication of monolayers of organic molecules with unpaired spins on a thin gold film. Nitroxyl 2,2,6,6-tetramethylpiperidin-1-oxyl (TEMPO) radicals and terpyridinyl-cobalt complexes were used in this study. The existence of unpaired spins in self-assembled monolayers was investigated by XPS, and CV measurements. XPS measurements identified the cobalt ions in the monolayer. The two main peaks occurred at 781.3 and $796.3 \mathrm{eV}$, corresponding to the $\mathrm{Co} 2 \mathrm{p}_{3 / 2}$ and $\mathrm{Co} 2 \mathrm{p}_{1 / 2}$ states, respectively. The CVs of cobalt complexes on gold substrate showed a typical reversible electrochemical reaction, with an oxidation peak at $0.22 \mathrm{~V}$ corresponding to the $\mathrm{Co}^{3+/ 2+}$ process. The CVs of TEMPO radicals exhibited a symmetrical reversible one electron redox process. The redox potentials were $+0.72 \mathrm{~V}$ and $+0.68 \mathrm{~V}$ vs $\mathrm{Ag} / \mathrm{AgCl}$, respectively. Electrical transport measurements of terpyridinyl-cobalt complexes showed an increase of the sheet resistance of gold layers covered with a SAM of magnetic and non-magnetic molecules when the temperature was decreased. Electrical transport measurements of TEMPO radicals were carried out and no significant difference in resistance between the bare gold and the gold covered with the monolayer was observed.

The results presented in this thesis illustrate the power of relatively weak supramolecular interactions to direct the immobilization of functional systems on surfaces. The work on the combination of host-guest and lanthanide-ligand coordination interaction motifs on surfaces demonstrates that hybrid, multifunctional supramolecular monolayers can be fabricated by integrating different non-covalent interactions in the same system. Hence, the combination of different supramolecular interactions opens up new avenues for the fabrication of complex hybrid organic-inorganic materials and stimuli-responsive surfaces. 


\section{Samenvatting}

Het in dit proefschrift beschreven onderzoek is gericht op de combinatie van orthogonale supramoleculaire interacties voor functionele monolaag-architecturen op oppervlakken. De term “orthogonale supramoleculaire interacties” verwijst naar nietcovalente interacties die elkaars organisatie-eigenschappen niet beïnvloeden als ze in hetzelfde systeem worden toegepast. Orthogonale zelf-assemblage geeft een uitgebreide controle over het zelf-assemblageproces en bevordert nieuwe materiaaleigenschappen. Individuele niet-covalente interacties (bv. waterstofbrug-, metaalcoördinatie-, elektrostatische of gastheer-gast-interacties) zijn in veel studies toegepast. Echter, de combinatie van verschillende supramoleculaire interacties in hetzelfde systeem kunnen de eigenschappen van de materialen verbeteren. Het in dit proefschrift beschreven onderzoek heeft als doel de ontwikkeling van hybride, multifunctionele monolagen. Door gebruik te maken van orthogonale supramoleculaire interacties kan de samenstelling van de monolaag en de functionaliteit gestuurd worden. In het eerste deel van dit proefschrift (Hoofdstukken 3 tot 5) zijn orthogonale gastheer-gast en lanthanide-ligand coördinatie-interactie-motieven gebruikt om supramoleculaire luminescente monolagen te maken. Het tweede deel van dit proefschrift (Hoofdstukken 6 en 7) behandelt de fabricage van functionele monolagen op silicium- en goudsubstraten voor toepassingen in elektronica.

Hoofdstuk 1 geeft een algemene inleiding die ingaat op het belang van nietcovalente interacties voor de fabricage van functionele oppervlakken.

Hoofdstuk 2 geeft een literatuuroverzicht over het gebruik van supramoleculaire interacties voor het maken van hybride assemblages en materialen. Supramoleculaire chemie en moleculaire zelf-assemblage, inclusief waterstofbrug-, metaalcoördinatie-, en gastheer- 
gast-interacties, voor de sturing van de verankering van functionele systemen op oppervlakken worden in detail besproken. Speciale aandacht wordt geschonken aan de combinatie van verschillende supramoleculaire interactie types voor het maken van functionele monolaag-architecturen.

Hoofdstuk 3 beschrijft de assemblage van een gast-gefunctionaliseerde naftaleenantenne en een $\mathrm{Eu}^{3+}$-complexerend, op EDTA-gebaseerd, ligandmolecuul op een receptoroppervlak door de combinatie van orthogonale gastheer-gast- en metaal-ligand-coördinatiemotieven. Door middel van lokale fluorescentie-emissiespectra van het geïmmobiliseerde complex werd de aanwezigheid van het gesensitiseerde $\mathrm{Eu}^{3+}$-complex gedemonstreerd. De efficiëntie van de energie-overdracht tussen de antenne en het $\mathrm{Eu}^{3+}$-complex werd aan de hand van tijdsopgeloste fluorescentiemetingen bepaald op ongeveer 35\%. Door het bestuderen van de gesensitiseerde luminescentie van het oppervlak-geïmmobiliseerde complex bij verschillende antenne-Eu ${ }^{3+}$-complex-verhoudingen, werd de stoichiometrie van de complexering van de antenne en het $\mathrm{Eu}^{3+}$-complex bepaald op 1:1 door middel van de allereerste Job-plot-analyse aan een oppervlak.

Hetzelfde systeem van lanthanide-complexen is in Hoofdstuk 4 gebruikt voor de verhoudingsgewijze detectie van dipicolinezuur (DPA), dat een unieke biomarker is voor bacteriële miltvuursporen, met een hoge gevoeligheid en selectiviteit op een supramoleculair monolaag-oppervlak. De fluorescentie-intensiteitsverhouding van het op lanthanidegebaseerde receptor-oppervlaksysteem toonden na toevoeging van verschillende DPAconcentraties aan dat het herstel van de naftaleen-emissie nagenoeg compleet is in de aanwezigheid van slechts 200 nM DPA. De verandering van de intensiteit verhouding van het op $\mathrm{Eu}^{3+}$-gebaseerde detectieplatform als functie van de tijd, na toevoeging van $200 \mathrm{~nm}$ DPA, wees erop dat het detectiesignaal binnen 10 minuten compleet is. De detectielimiet van het systeem is ook onderzocht en bepaald op ongeveer $25 \mathrm{nM}$ DPA. Om de selectiviteit van het 
supramoleculaire detectiesysteem aan te tonen, zijn de veranderingen in fluorescentie onderzocht na toevoeging van verschillende concurrerende aromatische liganden. De verwaarloosbare veranderingen toonden de uitstekende selectiviteit van het systeem voor DPA aan.

Hoofdstuk 5 introduceert een supramoleculair detectieplatform op een microchipoppervlak voor de detectie van biologisch relevante fosfaat-anionen en aromatische carbonzuren in een voor een hoge doorvoer. Het op $\mathrm{Eu}^{3+}$-gebaseerde supramoleculaire detectiesysteem werd geïmplementeerd aan het oppervlak van een meerkanaals-chip. De detectielaag op het microchip-oppervlak bevat een ensemble van de antenne en het $\mathrm{Eu}^{3+}$ gecomplexeerde ligand. Door de toevoeging van een anionische gast aan het ensemble werd, door het verdringen van de antenne, een vermindering in het fluorescentiesignaal veroorzaakt na binding met het $\mathrm{Eu}^{3+}$-ion. ATP en pyrofosfaat, naast verschillende fosfaat-anionen, en de miltvuur-biomarker DPA, naast verschillende aromatische carbonzuren, gaven een sterke respons op het gesensitiseerde $\mathrm{Eu}^{3+}$-luminescentie-gebaseerde microchip-oppervlak.

Hoofdstuk 6 beschrijft de lokale dotering van oxide-vrij silicium door middel van nanoimprint-lithografie (NIL) en moleculaire monolagen. Covalent gebonden Si-Cmonolaag-patronen in grootte variërend van $100 \mathrm{~nm}$ tot $100 \mu \mathrm{m}$ werden via een lokale hydrosilyleringsreactie op door NIL gepatroneerde gebieden gemaakt. Deze patronen werden gekarakteriseerd door middel van XPS, AFM en TOF-SIMS. Monolaag-patronen van $100 \mu \mathrm{m}$ werden gemaakt voor karakterisatie door middel van XPS. Element-specifieke plaatjes toonden aan dat de gepatroneerde monolagen gemaakt waren door hydrosilylering. Voor de fabricage van structuren op nanoschaal werd een nikkel- mal met een vierkante matrix van pilaren van $150 \mathrm{~nm}$ gebruikt. De resulterende structuren werden bestudeerd door middel van AFM, waarbij duidelijk gedefinieerde patronen zichtbaar werden. Aan de hand van een fosfor-houdende organische verbinding, werden sterk-gedoteerde gepatroneerde gebieden (op 
$\mu$ m-schaal) in nagenoeg puur, ongedoteerd silicium met succes gefabriceerd. Deze gepatroneerde gebieden werden gekarakteriseerd door middel van TOF-SIMS voor imaging en diepte-profilering. De dosis van het doteermiddel binnen een gedoteerd gebied werd bepaald op $(2.3 \pm 0.1) \times 10^{19} \mathrm{P}$ atomen. $\mathrm{cm}^{-3}$, hetgeen correspondeert met een oppervlaktedosis van $5.6 \pm 0.1 \times 10^{13} \mathrm{P}$ atomen.cm ${ }^{-2}$. Elektrische karakterisatie werd uitgevoerd met behulp van van Hall en vierkantsweerstandsmetingen. Een gemiddelde ladingsdragerdichtheid werd bepaald op $1.9 \pm 0.1 \times 10^{13} \mathrm{~cm}^{-2}$ bij $300 \mathrm{~K}$ en $9.4 \pm 0.1 \times 10^{12} \mathrm{~cm}^{-}$ 2 bij $150 \mathrm{~K}$ in het geval van gepatroneerde monsters. Belangrijker, op met lijnen gepatroneerde substraten was de gemeten weerstand in de lijnrichting aanzienlijk lager dan wanneer de weerstand loodrecht op het lijnpatroon gemeten werd.

In Hoofdstuk 7 wordt de fabricatie van monolagen van organische moleculen met ongepaarde spins op een dunne goudlaag beschreven. Voor deze studie werden nitroxyl 2,2,6,6-tetramethylpiperidin-1-oxyl (TEMPO) radicalen en terpyridinyl-kobaltcomplexen gebruikt. Met XPS en CV-metingen werd de aanwezigheid van ongepaarde spins in zelfgeassembleerde monolagen aangetoond. De aanwezigheid van kobaltionen in de monolaag werd bevestigd door XPS-metingen: de twee hoofdpieken bij 781.3 en 796.3 eV komen overeen met respectievelijk Co2 $\mathrm{p}_{3 / 2}$ en $\mathrm{Co} 2 \mathrm{p}_{1 / 2}$. De CVs van kobaltcomplexen op goudsubstraten toonden een typische reversibele elektrochemische reactie met een oxidatiepiek bij $0.22 \mathrm{~V}$, die correspondeert met het $\mathrm{Co}^{3+/ 2+}$ proces. De CVs van TEMPOradicalen gaven een symmetrisch en reversibel één-elektron-redoxproces weer. De redoxpotentialen waren respectievelijk $+0.72 \mathrm{~V}$ en $+0.68 \mathrm{~V}$ t.o.v.Ag/AgCl. Elektrische transportmetingen van terpyridinyl-kobalt-complexen gaven een toename van de vierkantsweerstand van de goudlagen die bedekt waren met een monolaag van magnetische en niet-magnetische moleculen bij verlaging van de temperatuur. Met elektrische transport- 
metingen aan TEMPO-radicalen konden geen significante verschillen tussen onbedekte goudsubstraten en goudsubstraten die bedekt waren met de monolaag worden aangetoond.

De resultaten die gepresenteerd zijn in dit proefschrift illustreren de kracht van relatief zwakke supramoleculaire interacties voor de sturing van de immobilisatie van functionele systemen op oppervlakken. Het werk aan de combinatie van gastheer-gast- en lanthanide-ligand-coördinatie-motieven laat zien dat hybride, multifunctionele supramoleculaire monolagen gemaakt kunnen worden door de integratie van verschillende niet-covalente interacties in hetzelfde systeem. Derhalve opent de combinatie van verschillende supramoleculaire interacties nieuwe wegen voor de fabricage van complexe hybride organisch-anorganische materialen en stimulusgevoelige oppervlakken. 


\section{Acknowledgements}

Finally I finished my $\mathrm{PhD}$ thesis, and I would like to thank many people for their help and support during these four years. I am very proud that now it is time to write the most important part of the thesis. It is not easy to summarize my all acknowledgements in a few words, but just I can say that I am really grateful to all people I met during my $\mathrm{PhD}$.

My first acknowledgement goes to my promoter and supervisor, Jurriaan Huskens. Actually I don’t know where I will start and I will never find enough words to say. I thank you for accepting me as a $\mathrm{PhD}$ student and to be part of this group. I will never forget our biweekly meetings, where I have learnt so much from you. During these meetings you always gave me lot of confidence, encouragement and freedom mostly when I lost my way. You were always patient to listen to me when I brought completely different ideas while proceeding a certain project:- I want to thank you for all your help, ideas, guidance, and patience throughout this whole period. Thank you very much for everything you did for me.

I would like to thank to Aldrik and Wilfred for the meetings we had in the last 3 years of my $\mathrm{PhD}$ and for the scientific contribution. They were very useful with a lot of nice ideas. I really enjoyed and learned so many things during our meetings.

During these four years I had the chance to collaborate with many people, the results of which are presented in this thesis. I owe my appreciation to Shu-Han Hsu for all her help. You always had time for me either to help me to prepare the patterned samples nor for discussions on results. We had many nice discussions which were very essential to obtain results presented in Chapter 3 and 4. I would like to thank Dr. Bilge Eker for introducing me to the world of microchips and for her participation in Chapter 5. I really learnt a lot from you. I would like to thank Pim for his contribution in Chapter 6. You were my first master 
student and I couldn't support you much during your studies due to my lack of knowledge about nanoimprint lithography. At the end, when we looked the results, we managed this project successfully. My appreciation also goes to Wouter Naber and Peter Tijssen for their help for Chapter 7. It was a long project and still continue with a new PhD student, Derya. I hope you will complete this project during your $\mathrm{PhD}:-$ I would like to thank all of you again, it was pleasure to work with you and without your help I could not complete my PhD. My special thanks go to Mudassir Iqbal. Dear Mudooo, thank you very much for all your friendship and help during these years. I will never forget our discussions the topic could be whatever. I appreciate your friendship. I would like to thank to Marcel and Richard for their technical support. The secretaries of MnF/SMCT/BNT, Izabel and Nicole, are also thanked for taking care of all administrative works. I thank you Melissa for going so carefully through my concept thesis. I also spent some time doing EPR measurements in Barcelona. Dear Veronica, thanks for your help and contribution on EPR experiments. I really enjoyed during my short trip to Barcelona. I would like to thank to Tom. Dear Tom, thank you very much for your great effort to translate thesis summary from English to Dutch. I appreciate your kindness. I would like to thank to Janet Acikgoz. Dear Janet, thanks for encouraging me in the first year of PhD. Whenever I was upset, you tried to recover me over and over again (:)

During my PhD, I have had opportunity to meet a lot of fantastic people, Xing Yi, Duan, Henk, Sachin, Veera, Elisabetta, Riccardo, Francesca, Ignacio, Andras, Huaping, Yiping, Srinidhi, Tian, Kim, Roberto, Albert, Arancha, Carmen, Sven, Carlo, Jordi, Lanti, Jealemy, Pieter, Alberto, Melanie, Raluca, Vijay, Chien-Ching Wu, Dae June, Oktay, Erhan, Serkan, Mine, Anna, Rajesh, Nicolai, and many more... All the people I mentioned here have contributed to make the time I spent in Enschede an unforgettable time of my life. But most importantly, I owe my deepest gratitude to my family; my parents, my two brothers for all the support they give me. Canim annem, babam, kardeslerim. Sizlerin sevgi ve anlayisiyla ben bu 
doktorayi bitiriyorum. Bana desteklerinizi hicbir zaman eksik etmediniz. Sizleri cok ama cok seviyorum.

Lastly, this thesis is dedicated to the owner of my heart, Burcu, I wouldn’t complete this thesis without you. You are my soul, my lover, and my best friend. My heart is full with your love. We have overcome all bad moments, and welcome every good moments hand-inhand in our life. We are growing and walking together to our future. I love you! Seni cok seviyorum askim herseyim.

Deniz Yilmaz

Enschede, The Netherlands

May 2011 


\section{About the author}

M. Deniz Yilmaz was born on $14^{\text {th }}$ April 1979 in Ankara, Turkey. He obtained his Bachelor degree of Chemistry, $1^{\text {st }}$ Class Honors from Ankara University, Turkey in the year 2003. He received his Master degree of Chemistry in 2006, with research project entitled "Light Harvesting and Efficient Energy Transfer in Boron Dipyrrin (BODIPY) Functionalized Perylenediimide Dyads” under the supervision of Prof. Dr. Engin U. Akkaya in Middle East Technical University. From June 2007, he was a PhD candidate under the supervision of Prof. Dr. Jurriaan Huskens in the Molecular Nanofabrication (MnF) group at the University of Twente, The Netherlands, on the project of the preparation of hybrid, multilayered materials with a complex architecture. The results of this research are described in this thesis. 